\title{
A controlled Study of Effectiveness and Patient suitability for Short-term Group Psychotherapy
}

By

\author{
Mary Mocallum
}

A thesis submitted to the Faculty of Graduate Studies and Research in partial fulfiliment of the requirements for the degree of Doctor of Philosophy.

Department of Psychology

MOGill University

Montreal

April 1989

(c) M. Mocallum 1989 
Controlled Outcome Study of Short-term Group Therapy 


\section{Abstract}

A controlled, clinical trial investigation of short-term psychoanalytically oriented group psychotherapy (STG) was conducted which included eight psychotherapy groups led by experienced therapists. Patient psychological mindedness (PM) was investigated as a selection criterion and prognostic variable. Seventy-nine psychiatric outpatients experiencing prolonged or delayed grief reactions were matched for level of $\mathrm{PM}$ and then randomly assigned to STG or to a wait list. There was repeated measurement of several areas and sources of outcome. Results indicated a strong main effect for STG but not for PM on outcome, and some evidence of an interaction effect. Benefits were maintained at six-month follow-up. Psychological mindedness emerged as highly predictive of attrition and moderately predictive of psychodynamic work. Psychodynamic work was monitored by process analysis ratings and was modestly related to outcome. Methodological Iinitations, clinical significance, clinical implications, and suggestions for future reseirah are discussed. 


\section{Sommaire}

Une étude clinique contrôlése portant sur la psychothérapie brève de groupe d'orientation analytique (PBG) fut effectuee au sein de huit groupes de psychothérapie dirigés par des therapeutes experimentés. Ia capacite d'introspection psychologique (CIP) fut étudiée en tant que critère de sélection et variable pronostique. Soixante-dix-neuf patients recrutés en clinique externe de psychiatrie, présentant des réactions de deuil prolongées ou retardées, furent pairés d'après leur niveau de CIP et assignés ensuite de façon aleatoire a un PBG ou à une liste d'attente. Differents aspects et sources de l'issue therapeutique furent mesurés à plusieurs reprises. Les résultats révèlent une influence principale importante sur l'issue thérapeutique de la part du PBG mais non du CIP, et mettent en évidence un certain degré d'interaction. Ie suivi a permi de constater que les bénéfices s'étaient maintenus au bout de six mois. Ia capacité d'introspection psychologique s'est avérée hautement prédictive quant à l'attrition, et modérément prédictive quant au travail d'élaboration psychodynamique. Ie travail d'élaboration psychodynamique, evalué sur la base de scores abtenus à l'analyse de processsus, s'est avéré modestement relié à I'issue thérapeutique. Ies limitations d'ordre méthodologique, les implications et les significations cliniques, ainsi que des suggestions pour la recherche à venir sont abordées. 


\section{Acknowledgements}

It is with great pleasure that I acknowledge the many people who contributed their time and expertise toward the successful completion of this research investigation.

I am profoundly indebted to $m y$ supervisor and mentor, Dr. William E. Piper. Throughout the many phases of our association he has always encouraged and fic litated my professional evolution. In addition to his sharing of expertise, I feel I have most benefited from the integrity and dedication with which he has guided the many complex facets of this study.

Special thanks to the project's oo-ordinator, Ms. Hillary Morin. Her dependability and organization when implementing each aspect of the study guaranteed its successful completion. I especially appreciate her patience and compassion when interacting with the study's patients.

My sincerest gratitude to the study's therapists, Ms. Fyfe Bahrey and Mr. William ifickerson. Their cooperation with the research requirements enabled the ideas to come to fruition. I also want to thank Imy dear friends, Ms. Jocelyne Iauzière and Mr. Carl Aboud for graciously agreeing to portray the patient-therapist dyad on the videotape.

I would like to extend my appreciation to the following members of the Program Evaluation and Research Unit, Division of Extemal Psychiatric Services, University of Alberta Hospitals: Jill Zimmerman, 
Doris Ryan, Susan Hurst, Nancy Hurst, Karen Evans, Jayne Carlielle, Bonnie Stephanson, and Ellen Jackson. In their roles as outcome and suitability assessors, process raters, and data tabulators, their conscientious attitude ensured the quality of the research. The contributions of statisticians, Mr. Daniel szeto in Ecmonton and Ms. Rhonda Amsel in Montreal, are also gratefully acknowledged. A special note of thanks to research associate, Mr. Anthony Joyce, for sharing his extensive library and experience with me, and for empathizing with my apprehensions throughout the study.

I also want to thank the entire staff of the walk-in Clinic of the Division of External Psychiatric Services, University of Alberta Hospitals. The constancy with which the therapists referred and prepared patients for the groups reflected their confidence and belief in the goals of the study.

I sincerely appreciate the participation of the study's patients. I feel honoured to have been entrusted with the most personal thoughts and feelings which constitute this study's de- . I also thank the volunteers who participated in the Montreal pilot study.

on a more personal note, I thank my husband, Mr. Donald MacAskill, for his many sacrifices, emotional support and encouragement throughout the years of this dissertation.

This research was supported by grant number 6609-142646 from the National Health Research and Development Program, Health and Welfare, Canada, and by a grant from the Canadian Psychiatric Research Foundation. Their financial assistance is gratefully acknowledged. 
Abstract. ..........................................

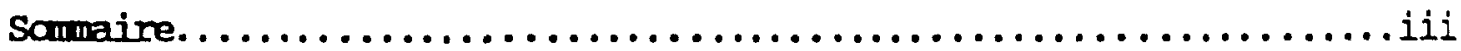

Acknowledgements.................................... iv

Table of contents....................................

Iist of Tables..................................

Introduction. $\ldots \ldots \ldots \ldots \ldots \ldots \ldots \ldots \ldots \ldots \ldots \ldots \ldots \ldots \ldots \ldots \ldots$

I. Review of STG Clinical and Research Literature..........7

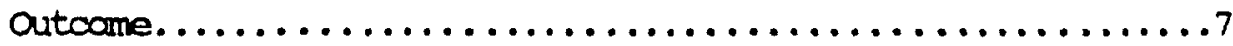

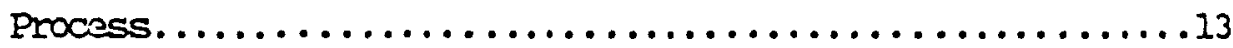

Fatient Selection...........................20

II. Psychological Mindedness

as a Selection Criterion for STG...................23

III. Rationale for the Present Study....................... 30

IV. Hypotheses..................................

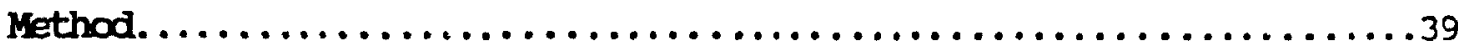

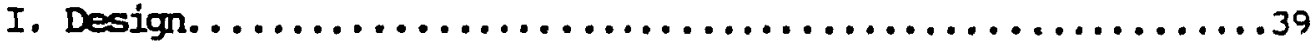

II. Patients. .................................. 42

III. Therapists.................................44

IV. Initial Clinical Interview (Intake) $\ldots \ldots \ldots \ldots \ldots \ldots \ldots \ldots 46$

V. Initial Research Interview.....................49

VI. Group composition............................

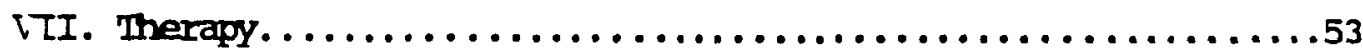

VIII. Follow-through Assessments...................... 55 
Ix. Measures................................... 57

1. Patient variables...........................57

A. Psychological Mindedness Assessment Procedure........ 57

B. Therapist-rated Patient Characteristics............69

2. Therapy Process..........................

A. Psychodynamic work and Object Rating system........70

B. Therapist-rated process Variables...............78

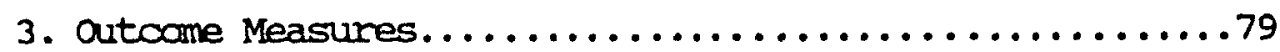

A. Social Adjustment scale.....................80

B. Tanget Objectives.........................82

c. Impact of Event Scale.....................84

D. Interpersonal Dependency Inventory............. 85

E. Interpersonal Behavior Scale.................

F. Rosenberg Self-esteem Scale..................88

G. Self-report Symptom Inventory $(S C I-90) \ldots \ldots \ldots$. . . . 89

H. Beck Depression Inventory.........................

I. Global Ratings of outcome:

The Life Satisfaction Scale, The Overall Usefulness of Therapy,

The Service Evaluation Questionnaire.............91

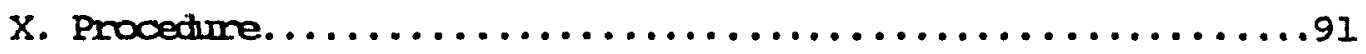

Results..........................................93

I. Patient. Samples Available for Analyses...............94

II. Pretest score Analyses.........................99

III. Tests of the Major Hypotheses........................

Hypotheses 1, 2, and $3 \ldots \ldots \ldots \ldots \ldots \ldots \ldots \ldots \ldots \ldots \ldots \ldots \ldots$ 
Hypothesis 1 - Supplementary Analyses.................106

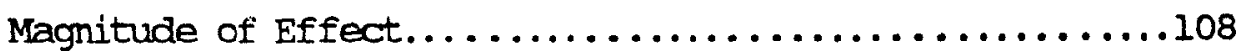

Clinical Significance.........................110

Delayed Treatment Phase Versus Wait-Tist Phase.........118 Hypothesis 2 - Supplementary Analyses..................20 Hypothesis 3 - supplementary Analyses....................

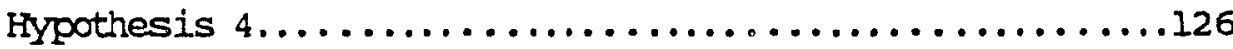

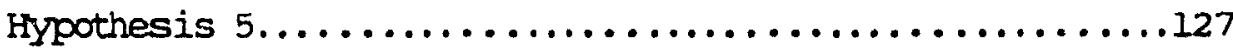

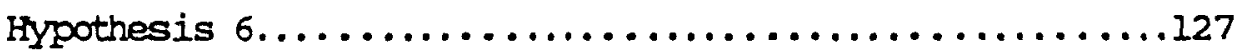

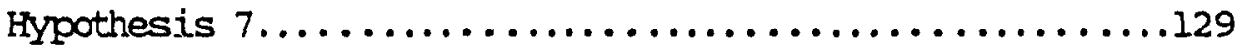

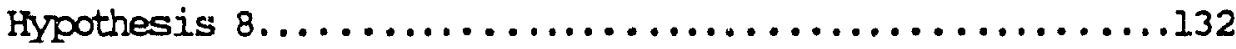

IV. Additional Analyses..............................

Therapist Effects.................................

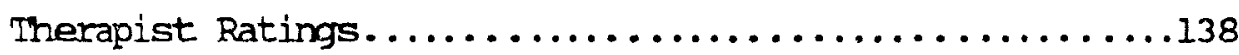

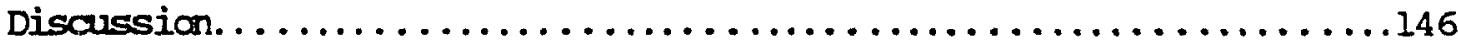

I. The outcome of STG..............................

The Treatment Effect...........................

quantitatively Detemined Clinical Significance..........149

The Nature and Etiology of Pathological Grief............15I

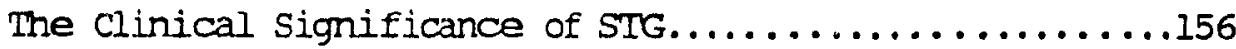

II. The Therapentic Process of SIG. .................... 158

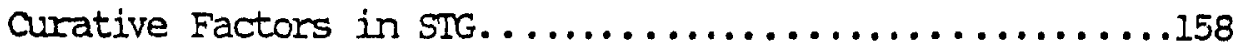

The Integrity of the Psychoanalytic Process...........165

Psychodynamic work as a Curative Factor in STG........167

Success of Alternative Treatments for Loss Patients......169 
page

Attrition..................................

III. Patient Suitability for STG................... 177

Psychological Mindedness as a Selection Variable........177

Interpretation comprehension as a

Predictor of SIG Process and outcome................181

Treating Marginally Psychologically Minded

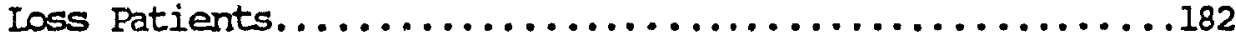

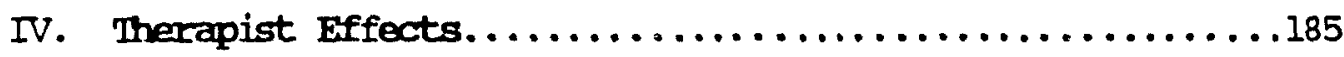

V. Iimitations and suggestions for Future Pesearch.........186

VI. Strengths and Implications..................... 188

VII. Original Cantribution. .......................... 192

Bibliography..........................................

Appendices.........................................206

Appendix A. Informing Referral Sources of the STG Program....206

Appendix B. Therapist Mamual.........................207

Appendix C. Guidelines for Referral to STG..............211

Appendix D. Patient Ground Rules for STG................214

Appendix E. Informed Consent Form......................... 215

Appendix F. Referral Fonn........................... 217

Appendix G. Procedure for Independent Assessment. . . . . . . . . 218

Appendix H. Mamual for the Psychological Mindedness

Assessment Procedure....................221

Appendix I. Mean and Standard Deviation

for PMAP variabies....................235

Appendix J. Therapist-Rated Patient Characteristics. . . ......236

Appendix K. Marual for the Psychodynamic Work and Object Rating System................240 
Appendix L. Definition of Patient Work Behavior

for Therapist Ratings.........................

Appendix M. Therapist-Rated Process Ratings................252

Appendix N. Outcome Measures.........................253

A. Social Adjustment scale................253

B. Target Objectives...................256

C. Irmact of Event scale.................259

D. Interpersonal Dependency Inventory.........260

E. Interpersonal Behavior Scale............262

F. Rosenberg Self-Esteem Scale. . . . . . . . . . 266

G. Self-Report Symptam Inventory (SCI-90) ......267

H. Beck Depression Inventory................269

I. Global Ratings of outcome.............271

Appendix C. 2 X 2 ANCOVA Tables: Main and Interactive Effects of Treatment and condition

on outcome.............................273

Appendix P. Mean and Standard Deviation of FWORS Ratios......278 
ITST OF TTABIES

Page

Table 1 Overview of the Design...........................40

Table 2 Overview of Measures and Time of Evaluation.............59

Table 3 Intracorrelation coefficients for PMAP Variables........69

Table 4 Summary of Patient Attrition.....................95

Table 5 Psychological Mindeiness and Attrition

During Treatment Phase...........................97

Table 6 F-Ratios From Two by Two Analyses of covariance

(ANCOVA) Indicating Main and Interaction Effects

of Treatment and suitability......................105

Table 7 Mean and t-Value of Prescore to Postscore Change

on outcome Variables for Patients in the

Inmediate Treatment condition.......................107

Table 8 Mean and $t$-Value of Prescore to Postscore Change

on outcome variables for Patients in the control

condition......................................109

Table 9 Specific and Aggregated Effect Size and

corresponding Area Percentage of Normal curve

for Treated Versus Control Patients..................111

Table 10 Clinical Significance of Treatment Effects

Utilizing Normative Comparisons..................115

Table 11. Clinical Significance of Treatment Effects

Utilizing the Two Standard Deviation Criterion

Since Norms were Unavailable..................117

Table 12 Mean and $t$-Value from correlated $t$-tests on

outcome at Three Temporal Stages for Delayed

Treatment Patients............................119

Table 13 Correlation coefficients for Interpretation

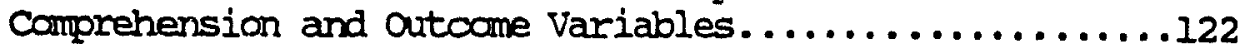

Table 14 cell Means of Adjusted Postscores of

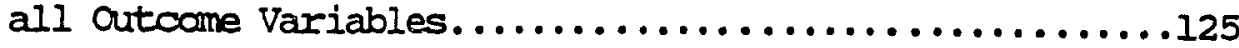


Table 15 Summary of Rank Ordering on Outcome Variables

Based on the Adjusted Postscore Cell Means............126

Table 16 Pearson Correlation Coefficients for FMAP with

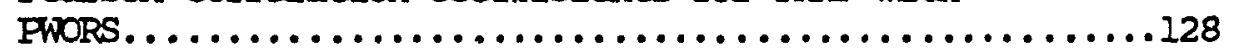

Table 17 Correlation Coefficients for FWoRs Ratios

and outcome variables................................

Table 18 Mean and $t$-Value of Posttreatment to Follow-up

Change on outcome Variables for Treated Patients........133

Table 19 Clinical Significance of Treatment Effects

At Six-Month Follow-up Utilizing Normative

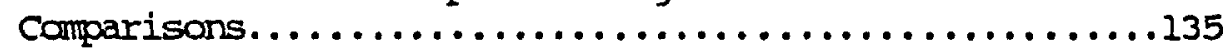

Table 20 Clinical Significance of Treatment Effects At

Six-Month Follow-up Utilizing the Two Standard.

Deviation Criterion Since Norms were Unavailable.......136

Table 21 Correlation coefficients for Therapist-rated

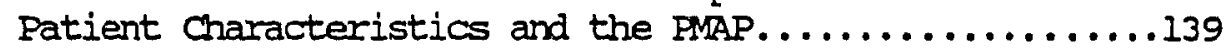

Table 22 Correlation coefficients for Therapist-rated

patient characteristics and outcome................14I

Table 23 Correlation coefficients for Therapist-rated

Process and FWoRs Variables...................... 142

Table 24 correlation coefficients for Therapist-rated

Process and outcome variables.....................144 
A review of the group psychotherapy research literature of the past three decades reveals two notable trends. The first trend is increasing confidence among researchers in the efficacy of group treatment methods. The second trend is increasing sophistication of research techniques. These two trends are related. As group treatments continue to be associated with client improvement, the research focus shifts from the general question concerning the efficacy of group treatments to specific questions concerning which dimensions of group membership and group functioning contribute to therapeutic outcome. As researchers address the more sophisticated questions of group membership and group functioning, they develop more sophisticated research techniques. It is important to emphasize that investigations of these other dimensions maintain a strong focus on outcome. While an investigation may focus on a particular dimension of group therapy, the trend is towards understanding and/or predicting the interaction between patient predictor variables, group treatment process variables and outcome variables.

This comprehensive approach to the outcome question is consistent with suggestions made by reviewers of the group psychotherapy research literature. Kaul and Bednar (1986) recently concluded that "group treatments have been associated with client improvement in a variety of settings" (p.672) but that the conditions under which group psychotherapy can be effective are basically unknown and in need of systematic research. Similarly, Parloff and Dies (1977) stated that the question of group efficacy should be as follows: "What kinds of changes are produced by what kinds of interventions provided by what 
kinds of therapists with what kinds of patients/problems under what kinds of conditions" (p.316). Hence, researchers are cautioned that particular treatment methodologies may interact with special patient characteristics to determine psychotherapeutic outcome. The recommendation that investigators integrate patient predictor variables and treatment process variables into their investigations of outcome, addresses two familiar criticisms of the group field.

The first criticism involves the lack of integration between research and theory. Despite the consensus that group therapies are a cost-effective form of therapy for outpatients, Kaul and Bednar (1986) pointed out that there is a "conceptual malaise regarding the primary, unique, and defining characteristics of group treatments" (p.673). The recommendation that irvestigators elucidate the conditions under which group therapy is effective, addresses this first criticism by requiring, a priori, conceptual and operational definitions of the essential elements of group psychntherapy. These definitions can provide the conceptual foundation for the evolution of a theoretical integration of patient predictor variables, group treatment process variables and outcome.

The second familiar criticism of group psychotherapy research involves its lack of clinical relevance. Dies (1983a) reported that 77 percent of clinicians surveyed, questionned the relevance of group research. A particular weakness identified by many clinicians is the failure of many studies to integrate process and outcome. Hence, the crucial clinical question of effective technique has been rarely addressed. Researchers' failure to integrate process and outcome is 
probably due to Iimited resources needed to fulfill rigourous standards of "sufficiently lange samples of patients, grouns, therapists and methods" (Frank, 1975). The split between clinical research and practice tends to perpetuate the lack of relevance of the research and simultaneously prevents the development of basic concepts and propositions based on careful observation of group process.

clearly, an integration of research, theory and practice is necessary for the implementation of comprehensive investigations that address clinically relevant questions, that evolve from a secure theoretical foundation and which utilize methodologically sophisticated designs. The fragmentation of clinical research, theory and practice is particularly dangerous in this time of heightened sensitivity to therapeutic accountability. Parloff and Dies (1977) warned that "the group psychotherapist will be called upon increasingly to document that what he does is not simply useful but more simply useful than other available treatment approaches with specific patient categories" (p.282)

The practice of brief group therapy is one area that addresses the accountability challenge and promises an integration of group theory, research and practice. During the last decade considerable clinical activity has emerged concerning this form of treatment. Toseland and Siporin's recent (1986) review of the clinical and research group literature revealed that approximately two-thinds of the studies published between 1965 and 1985 concerned the use of group therapies of less than twenty sessions. The practice of brief group therapy addresses the challenge of therapeutic accountability by considering 
economic and conceptual factors. Economic factors include an interest in providing cost-effective treatments given the ever-increasing imbalance between available psychotherapists and the demand for services. In addition, there is the growing necessity of accomodating to limits set by third-party payment sources such as goverrment health care programs and insurance companies. Finally, there are activities such as supervision and training that compete for clinicians' time. Conceptual factors include the idea that time pressure accelerates the pace of therapeutic work and the idea that a time limit prevents long-term dependency by the patients. In addition, the success of brief individual treatments has encouraged experimentation with less traditional forms of group therapy.

Practitioners utilizing a client-centered, behavioral or cognitive-behavioral approach have quickly adapted their therapies to the group modality. Of less prevalence are short-term group therapies utilizing the psychoanalytically-oriented approach (STG). The objective of STG is to help patients solve their presenting problems by achieving insight into how their difficulties are related to unresolved intrapsychic conflicts and by initiating a process of working through that will continue beyond the treatment sessions. The achievement of this objective is believed to we facilitated by a therapy process characterized by psychoanalytic work. Psychoanalytic work has been defined by Bienvemu, Piper, Debbane, and de Carufel (1986) as entailing a regressive process, a teunical process, and a progressive process. The regressive process refers to the passive encouragement by the therapist of regression on the patient's part and the patient's 
acceptance of that regression. The technical process refers to the neutral position of the therapist, the centrality of the analysis of transference, and the use of interpretation as the main technique. The progressive process refers to the acceptance by the patient of working with interpretation.

Unlike the overall group psychotherapy research literature, the issue of STG efficacy remains unsettled. Clinical enthusiasm for the efficacy of STG is inconsistent with the research literature wherein only a few outcome studies are reported. This paucity may result from the fragmentation of clinical research, theory and practice. With respect to the fragmentation between the research and practice of STG, Poey (1985) observed:

at the present time there is a paucity of rigorous outcome research available on short-term dynamic group psychotherapy even though many such groups are being run. Perhaps this is because these groups are usually rum in high-volume clinics where little time is budgeted for research efforts (p.332).

With respect to the fragmentation between the theory, research and practice of STG, there are extensive conceptual confusion and debates in the literature concerning fundamental issues. The fundamental issues that remain unsettled include whether or not one can carry out psychoanalytic work on an on-going basis in a short-term group format. Other unsettled issues concern patient selection criteria and therapist technique. Hence, uncertainty involving fundarnental issues of STG membership and treatment process has probably hindered the implementation of controlled studies of STG. 
Given the interplay between the various components of group treatment, the manner in which researchers resolve one issue will have implications for other components and ultimately, will have a direct bearing on the results of an outcome investigation. A corollory is also true: uncertainty concerning the efficacy of STG (partially due to the paucity of outcome studies) can affect how researchers approach the resolution of the unsettled issues concerning group membership or therapy process. For example, doubts about the possibility of affecting structural change (i.e. profound changes in characterological and/or psychic structure) seem to have contributed to the generation of a number of other objectives tinat have been mentioned in the literature. They include symptam reduction, crisis resolution, support, trial therapy, student training and preparation for long-term therapy. Such objectives can be important and compared to structural change, may be more readily attainable. However, their pursuit can serve to deplete the resources of a therapy group and diffuse the therapist's technique. In the end this diffusion can diminish the chance of accomplishing psychoanalytic work and affecting structural change. Hence, a self-fulfilling prophecy can occur where doubts about the efficacy of STG can lead to confusion concerning treatment objectives, which in turn, can hinder clarity concerning psychoanalytically oriented therapist technique, which finally, results in outcomes which support doubts about the efficacy of STG.

In response to the need for basic controlled studies of STG, the primary objective of the present study was to conduct a controlled, clinical-trial investigation of STG. Consistent with recommendations 
in the literature, the present investigation was also designed to address aspects of group membership and group functioning believed to influence the outcome of SIG. The issue of group membership addressed was that of patient selection. The issue of group functioning addressed was that of psychoanalytic work.

The remainder of this introduction is divided into four major sections. In the first section, there is a review of the clinical and research literature on short-term psychoanalytically oriented group therapy. This section is divided into three subsections. The first subsection reviews the STG outcome Iiterature. The second subsection reviews the STG therapy process literature. The third subsection reviews the STG patient selection literature. This is followed by a literature review of the patient dimension, psychological mindedness. Level of psychological mindedness represents a possible patient selection criterion for STG. The third section summarizes the rationale and description of the present study. The major hypotheses are presented in the fourth section.

\section{Review of STG Clinical and Research Iiterature}

\section{outcome}

A review of the literature on STG reveals a conmon yet ambivalently held viewpoint that one should not expect too much of short-term group therapy in terms of treatment outcome. For example, when commenting on their experiences with SIG for genital herpes sufferers, Drob and Bernard (1985, 1986) gave somewhat contradictory reviews. In one 
article they stated:

[i]t should be noted that most participants are not referred for further treatment immediately following the completion of the group, which suggests that the 10-week program, though quite short, is usually experienced as a complete treatment experience rather than a beginning which necessarily requires further elaboration (1985, p.19).

In a subsequent article, however, they tempered their enthusiasm by stating that "[a] time-limited psychotherap group cannot effect fundamental changes in character, but it can point the way to the long-term issues that must be addressed" (1986, p.140). It is not clear whether they believed that the long-term issues must be addressed in subseguent therapy.

Drob and Bernard's tempered enthusiasm is consistent with other advocates of STG. Budman and Gunman (1983) argued that brief therapy can be usefll to everyone if it is not seen as a cure-all. Klein (1985) appears to be in agreement when he said that in STG it may be possible to identify core conflicts and to examine interpersonal implications, but not to work through core conflicts or achieve lasting structural change. Goldberg, Schuyler, Bransfield, and Savino (1983) stated that helping patients appreciate the complex and conflictual nature of their experience is a realistic goal but completely resolving their problems is not. Imber, Iewis, and Ioiselle (1979) also indicated Iinitations in the outcome of short-term groups by noting that many patients seek additional treatment when their groups are finished.

The work of Budman and his colleagues in Boston represents an 
optimistic point of view concerning the efficacy of STG. They offer an important contribution to the validation of STG as a viable treatment altemative. They have developed an acult developmental model for STG. Their groups are homogeneorlsly composed according to the patient's age and associated stage of development. There are young adult groups for those in their twenties, midlife groups for those between thirty-five and fifty, and later midlife groups for those over fifty. Consistent with the writings of E. Erikson (1950), each stage of life is assumed to confront an individual with particular concerns and conflicts that need to be negotiated and mastered. Hence, each type of group is focused around a particular area of conflict. The young adult groups focus on conflicts of intimacy versus isolation. The midlife groups focus on conflicts of stagnation versus generativity. The later midlife groups focus on conflicts of despair versus ego-integrity. Between six and ten members compose each group which are co-led by experienced therapists. The young adult groups and the midlife grours have a closed membership and meet weekly for fifteen sessions of ninety mimutes duration. Due to the issues associated with patients in the later midlife groups, these groups are open-ended. The therapists are active and focus on here-and-now interpersonal group behavior relating to the particular area of conflict associated for each type of group (Budman, Bennett, \& Wisneski, 1981).

In an article authored by Budman, Demby, and Randall (1980), the resilts of an uncontrolled outcome study with twenty-two groups were presented (192 patients were dispersed to 17 young acult groups and five midlife groups). The patients were assessed before and after 
treatment and at a four-month follow-up interview. Areas assessed included individually-formulated target objectives, psychiatric symptomatology, and attitudes towards the group. The results indicated that 32 patients dropped out before the fifth session, 41 were considered to be high changers, or to have received much benefit, while only ten patients were considered to be low changers, or to have received little benefit from the group experience. This study is offered as support for the efficacy of STG since the majority of the patients were able to benefit, at least to some degree, from a short-term group experience of the Budman model.

Apparently encouraged by these results, the Budman group proceeded to conduct a controlled outcome study of two young adult groups and a comparable number of waiting-list control patients (Budman, Demby, Feldstein, \& Gold, 1984). The measures utilized were the same as in the previous study. The results indicated that at the end of therapy, treated patients had improved more than the control patients. These results were, however, no longer statistically significant at the four-month follow-up period. This is the only controlled outcome study reported in the literature. It is an -mportant model for subsequent research in the area since the authors described their population, theoretical orientation and therapist technique as carefully as their research protocol. The fact that their population evidenced rather mild symptomatology (presenting with problems in living), may have interfered with finding even stronger treatment effects. This fact and the rather small number of patients participating in the study restrict the generalizability of the results. 
Iapointe and Rimm (1980) reported the results of a comparative outcome study. The study compared the efficacy of insight-oriented, cognitive and assertiveness therapies of reactive (situationally related) depression in women. There were two groups conducted utilizing each approach. Between three and nine patients composed each group (33 patients participated). The groups met weekly for six sessions of two hours duration. An experienced therapist individually led each group. The patients were assessed before and after treatment and at a two-month follow-up interview. Areas assessed included depression, irrational thinking and assertiveness, which was measured both subjectively and abjectively. The results indicated that all groups improved in all areas regardless of approach with the benefits being maintained at follow-up. This study supports the overall but not the differential efficacy of STG. The lack of differential efficacy of treatment approaches may be related to the methodological weaknesses of the study. These weaknesses include the rather low number of patients participating in the study. In addition, the insight-oriented approach is described by the authors as being rather structured, directive and cognitive. Hence, the therapies may have been more similar than different, with the insight-oriented approach being quite different from how it is usually conducted.

Another comparative outcome study of STG was reported by Piper, Debbane, Bienvenu, and Garant (1984). This study compared four forms of therapy: long-term individual, long-term group, short-term individual, and short-term group. The approach for all forms was psychoanalytically oriented. The long-term therapies lasted for two 
years while the short-term therapies lasted for six months. All sessions were conducted on a weekly basis. The individual therapy sessions were of 50 minutes duration while the group therapy sessions were of 90 minutes duration. Each of three experienced therapists conducted all forms of treatment. The patients were assessed before and after therapy and six months following therapy. Patients receiving the long-term therapies were assessed three additional times during treatment (after six, twelve and eighteen months of therapy). Areas assessed included interpersonal functioning, psychiatric symptomatology and personal target abjectives. The three sources of evaluation were the patient, the therapist and an independent assessor.

The results of the piper et al. (1984) study indicated that long-term group therapy and short-term individual therapy were superior to long-term indivichal therapy and short-term group therapy, in terms of outcome, therapy process and cost-effectiveness. In particular, "[e]xamination of the follow-up mean scores indicated almost without exception that STG had the poorest scores" (p.275). The authors contimued, however, to point out that "it would be a mistake to view its [STG] outcome effects as disastrous. Evidence for the negative effects was minimal . . . If STG therapy had been studied alone its outcome results would have seemed more favorable" (p.275). In considering why STG did so poorly compared to the other forms of therapy, the authors characterized the process as being one where "[i]nitia]. anxiety about working on sensitive issues in the presence of others was soon followed by anxiety about ending the group" (p.277). Hence, these authors suggested that perhaps "the structure of these 
groups would be more suitable for other orientations of short-term group therapy, for example those that consist of highly structured exercises and/or attempt to minimize rather than arouse anxiety during the sessions" (p.277). The relatively poor outcomes of STG in the Piper et al. (1984) study, may be attributable to the traditional manner in which the groups were conducted. Conversely, advocates of STG identify soveral guidelines when adapting psychoanalytically oriented therapy for the short-term group format. Piper et al. (1984) also stated that it is "possible that a different technical application of psychoanalytically oriented therapy with short-term groups would prove to be more successful" (p.277).

In summary, the outcome literature on STG is scarce revealing only one study which involved a no-treatment control group. The two studies that compared STG with other forms of treatment failed to support the differential efficacy of STG. In both these studies difficulty adapting the psychoanalytically oriented approach to the short-term group format is evident. The next section summarizes recormendations and guidelines that have been offered by proponents of STG concerning therapy process.

\section{Process}

The most fundamental issue revealed by a review of the STG literature is the uncertainty about carrying out psychoanalytic work on an on-going basis in short-term groups (MoCallum \& Piper, 1988). Given the demanding nature of this type of work under even the most optimal conditions, it is reasonable to wonder whether it is possible to 
accomplish if both the time for therapy is limited and the situation involves a group of patients. While a group is a natural place to experience regressive processes, such processes can be quite frightening for patients. coupled with inevitable group tensions concerning control, individuality, understanding, privacy and safety, strong resistance to interpretive work can be expected as a matter of course.

This uncertainty is reflected in an article by Herman and Schatzow (1984). In their work with incest victims, these authors argued that a group is particularly useful in helping their patients resolve issues of secrecy, shame and stigma. They qualified their argument, however, by stating that due to "the stressful and disorganizing nature of the group experience . . . members might need the protection of ongoing [individual] therapy . . [which can] help integrate the experience after the group [has] ended" (p.607). This qualification seens to reflect their uncertainty about the efficacy of a short-term group format. With respect to the psychoanalytically oriented approach, these authors seem simiiarly uncertain. They reported that their group therapist utilized a supportive orientation while "interpretations of group dynamics, individual resistances or unconscious material was [sic] avoided, except in cases where such an interpretation was necessary to return the group to its main focus" (p.614). Hence, while they were clearly familiar with the concepts and efficacy of the psychoanalytically oriented approach to group therapy, they seemed to utilize its techniques only as a last resort.

The uncertainty conceming STG is similarly reflected in the work 
of Bilodeau and Hackett (1971). In their work with myocardial infarction patient groups, these authors reported that the two most frequently expressed issues voiced by the group members involved the leader and group cohesion.

The predominant feelings expressed were fear and anxiety. To cope with these and other feelings of aggravation, anger, dependency, sadness, inadequacy, and shame the members used various observable techniques: joking, changing the subject, displacement, projection, denial, rationalization and identification (p.584).

Despite these authors' obvious familiarity and belief in psychoanalytic concepts, they advocated a therapist technique which gave "little interpretation to the material discussed" (p.584).

Ohlmeier, Karstens and Kohle (1973) also conducted short-term psychotherapy groups with myocardial infarction patients. These authors asserted, however, that due to this population's "extreme achievement problems [and] their fear of loss of identity and passive surrender, the analytic group situation . . . can be of valuable help" (p.241). Their approach reflects the belief that "[t]ransference, resistance, and communal unconscious phantasies are seen to be a product of the whole group. - . . Following from this, the interpretations of the therapists are always directed towards the group as a whole" (pp.241-242).

Those who have implemented psychoanalytically oriented techniques in their model of STG seem equally uncertain about its feasibility. Their uncertainty is reflected in debates concerning technique, for example, the use of transference interpretations. The model presented 
by Goldberg et al., (1983) "makes considerable use of transference toward the leaders" (p.414). Conversely, Budman and Bennett (1983) stated that "[t]ransferential issues and characteristic modes of dealing with authority figures may certainly arise within the context of the group; however, to allow these to become the central themes is not productive" (p.139). The recommendations of poey (1985) reflect his ambivalence in that he encouraged "rapid transference explorations . . from all members" (p.343) yet advocated:

the ideal is to keep the transferences to the leaders as positive as possible .. . [suggesting that] the leaders can easily sidestep negative transferences with direct, clarifying comments followed by suggestions to move back to the here and now work at hand" (P. 347).

These debates are reminiscent of the development of short-term psychoanalytically oriented individual therapy (STI). For example, there were the beliefs that transference interpretations could not be made early in therapy and that a gradual, prolonged period of working through was a requirement of effective therapy. The practice of STI, however, is currently very prevalent in the literature (for example, Strupp \& Binder, 1984; Bauer \& Kobos, 1987). In developing STI, advocates formulated conceptual and theoretical bases for their therapies. For example, Mann (1973) posited that brief time-limited therapy was ideal for addressing issues of separation by focusing on the horror of time (i.e. the existential anxiety aroused when facing the final separation, death). Hence, Mann identified stages of therapy which correspond to the idea of the horror of time being re-enacted 
within the course of therapy. He further identified the implications of these stages in determining therapist technique. Examples in the literature of the successful transition of psychoanalytically oriented therapy to the short-term group format reflect an adaptation of the technique. While there are many debates and disagreements concerning aspects of this adaptation, there is, nevertheless some consensus.

Advocates of STG agree upon the following technical elements: the encouragement of rapid group cohesion, the maintenance of a clear and specific focus, an emphasis on the awareness of the time limit, an active therapist role, and a focus on current relationships and behavior (particularly as they cocur in the group). Strategies for facilitating these technical elements include the delineation of stages of group development and the recommendation for homogeneous group composition. With respect to the first strategy, in delineating stages of group development, authors have used different nomenclature. Common stages identified, however, include the beginning and the ending stages with a working stage between them (Poey, 1985; Goldberg et al., 1983; Mackenzie \& Livesley, 1983; Drob \& Bernard, 1985). When advocating their model of group development, Mackenzie and Livesley (1983) posited that "the value of the developmental model for brief groups is that it directs the therapist's attention to events critical for the rapid estabiishment of . . [a mature] working system" (p.102).

With respect to the second strategy for facilitating the aforementioned technical elements, the groups are typically composed according to "broad-based homogeneity, including the establishment of a common theme" (Goldberg et al., 1983, p.423). Broad-based homogeneity 
refers to both patient qualities, for example, ego-strength, and to demographic characteristics, for example, age, sex, or marital status. The common theme can be defined in terms of several kinds of events. It can refer to a shared symptomatic pattern (e.g., depression), a shared historical event (e.g., the loss of a significant other), or a shared unconscious conflict (e.g., autonomy versus intimacy). Bucman et al. (1981a) composed their groups according to commonalities at more than one level of abstraction. As previously described, they chose patients who were at a common developmental stage, such as young adulthood, and who were assumed to be also experiencing difficulties concerning the conflict of intimacy versus isolation. Similarly, Drob and Bernard (1985) chose patients suffering from genital herpes and who were assumed to be experiencing conflicts about sexuality, intimacy and parenthood. This approach to composition is consistent with psychoanalytic principles in that "[I]ike any stressor, herpes can serve to highlight difficulties in an individual's general mode of adaptation that have obstructed successful living long before the disease was contracted" (Drob \& Bernard, 1986, p.140). The value of composing groups according to a common theme is that is provides a focus for the work and facilitates the development of cohesion.

It should be noted, however, that while practitioners of STG advocate composing groups according to a common theme, there is much disagreement concerning its level of abstraction. If the common theme is conceptualized at the unconscious level, the range of suitable patients is brozi.ter. The risk with this approach is that patients may have difficulty identifying with each other due to the heterogeneous 
manner in which the unconscious theme manifests itself. This may be so despite the clarity of the commonality in the therapist's mind. If the conmon theme is conceptualized at a more conscious level, such as symptomatology, the group process may remain at a superficial level. Interpretation and related psychoanalytic work may be difficult due to the variety of unconscious conflicts that give rise to similar manifest content. Klein (1985) cautioned that any homogeneously composed group may be more limited in terms of the range of interaction, inquiry and self-disclosure that occurs. Pragmatically, a group that is homogeneous with respect to a conmon theme is likely to be quite heterogeneous with respect to many other dimensions.

In summary, the successful transition of psychoanalytically oriented group therapy to the short-term format has demanded conceptual and techuical modifications. There remains uncertainty concerning the success of this transition in terms of conducting psychoanalytic work on an on-going basis in these groups. In particular, aspects associated with therapist technique and the level of abstraction for detemining a common theme contirue to be debated. Nevertheless, these modifications integrate aspects of therapy process, outcome and patient selection. For example, the delineation of a cormon theme can detemine treatment goals in that while the entire range of conflicts troubling patients cannot be addressed in a short-term group, except in the most superficial manner, the thorough exploration of a single common theme can be accomplished. With respect to outcome, the thorough exploration of a single conflict area can benefit patients indirectly by offering them an important exampie or model for 
understanding concurrent or subsequent conflicts in their lives. With respect to patient selection, patients should be selected according to these common themes. The next section presents a review of the STG patient selection literature.

\section{Patient Selection}

There are two main approaches to the development of patient selection criteria for STG. The first approach is associated with group composition strategies, as outlined in the previous section. This approach argues that some patients may have problems that are particularly conducive to work and resolution in brief time-limited group therapy. The second approach is associated with the psychnanalytic orientation of the therapy. This second approach argues that some patients may have attributes or qualities that are particularly suived to psychoanalytic work, especially in a short-term group format. Both of these approaches will be considered in this section.

With respect to selecting patients according to a common theme, Goldberg et al. (2983) pointed out that "there seems to be no specific limitation . . as long as it can be conceptualized as containing conflicts that can be worked with in the time allotted" (p.417). These authors identified three strategies for selecting a patient population: according to problems commonly found in an outpatient psvchiatric clinic (e.g., repetitive failures in relationships), problems commonly found in a general hospital (e.g., post-mastectomy recovery), or problems commonly found in the community (e.g., single parenthood, 
divorce). In addition to individual adults, STG has also been conducted with alcoholic couples (Mathiasen \& Davemport, 1988) and with early latency-age children (Charach, 1983). Within each homogeneous patient population, however, there exist differences with respect to suitability for STG. In other words, while patients may have problems that are particularly conducive to a short-term group format, not all patients with the same problem will be equally suited to work within the psychoanalytically oriented approach.

This variability in suitability is demonstrated in the work of Drob and Bernard (1986). In conducting short-term groups for genital herpes patients, they utilize a psychodynamic approach (DOP) and a cognitive-behavioral stress management approach (CBSM). The two approaches have several elements in common. The structure of both types of groups consist of ten weekly sessions of 85 mimutes duration. Many of the hypothesized curative factors are the same and include relief from isolation, overcoming denial, resolving ethical dilemas and exchange of information. With respect to selecting patients for each approach, the authors reported that the CBSM is offered to a wider range of patients, and is especially useful for relatively fragile individuals. The authors did not specify which patients are offered DOP. They did report that "the success of the DOP group is more variable and is dependent on such factors as . . the willingness of group members to take interpersonal risks in a group setting" (p.19).

The impression of Drob and Bernard concerning the variability of outcome of patients treated with STG is commonly reported in the literature. In the previously described outcome study by Lapointe and 
Rimm (1980), the authors concurred that "the insight group appeared to have the greatest variability in treatment outcome" (p.319). Similarly, in the study by Budman et al. (1980), while most patients received some benefit from the group, there was variability in the degree of benefit. The 41 patients who particularly benefited, were described as being in the neurotic range, and "although they come into the groum hurting and symptomatic, they begin treatment with a liking for people, friendships and a base from which to operate when interacting in the growp" (p.14).

There exists some consensus in the literature concerning suitable candidates for SIG. Poey (1985) outlined these common guidelines: an ability to verbalize a focal complaint, a significant level of psychological mindedness, an urge to grow and explore, a desire to enter STG, realistic expectations of the group and a hasic ability to relate and to be influenced by others. Despite a consensus among clinicians as to which patient qualities ought to predict outcome, "there is a continuing paucity of research data regarding the identification of individuals who will benefit from either long-term or short-term group therapy"' (Klein, 1985, p.31.3).

Woods and Melnick (1979) agreed with Klein by pointing out that the ability to predict outcome based on pretherapy dyadic assessments of patient diagnosis, personality dynamics or interpersonal factors is generally poor. one notes that the relationship between patient predictor variables and outoome is consistently poor throughout the psychotherapy literature. For example, in their work with individual psychotherapy, the Penn Psychotherapy Project reported generally 
insignificant results with only five to ten percent of the outcome variance being accounted for by some 80 predictor variables (Iuborsky, Mintz, Auerbach, Christoph, Bachrach, Todd, Johnson, Cohen, and O'Brien, 1980). In attermpting to explain the generally insignificant results of these studies, some authors have raised methodological concerns such as the outcome criteria utilized (MaNair, 1976) and the heterogeneity of the therapies involved (Bachrach, 1980). Another weakness of these studies involves the conceptual and operational definitions of patient dimensions utilized as patient predictor variables. The development of valid selection criteria for STG should primarily be based on considerations of concepts that are relevant to this form of therapy. The following section presents the rationale for operationalizing the concept, psychological mindedness, as a possible selection criterion for STG.

\section{Psychological Mindedness as a selection criterion for STG}

The goal in developing valid selection criteria involves determining the suitability of particular individuals for beneficial group treatment, that is, those who can be included and those who should be excluded. The relevance of identifying a suitable patient population for STG is evident. If group therapists accepted only those patients who are the most likely to benefit, they could minimize wasted manhours of therapists and financial and emotional costs to patients. Hence, the development of valid selection criteria clearly relates to the efficient utilization of available therapeutic resources. 
The researcher's challenge in developing valid selection criteria is "empirically identifying the group relevant behaviors requisite to achieving positive outcomes and then developing reliable and predictively valid pretherapy measures of these behaviors" (Woods and Melnick, 1979, p.162). Theoretically, the achievement of positive outcomes in STG depends on the patient's ability to contribute to the therapeutic objective. As previously mentioned, the objective of STG is to help patients solve their presenting problems by achieving insight into how their difficulties are related to unresolved intrapsychic conflicts and by initiating a process of working through that will contimue beyond the treatment sessions. The therapist uses an interpretive, here-and-now approach that focuses on unconscious conflicts. Specifically, the therapist endeavours to foster insight concerning how patients' presenting complaints are actually the manifestation of an underlying psychic conflict between umpermissable wishes, the anxiety or fear to which these wishes give rise and the ineffectual defense mechanisms that are mobilized to cope with the anxiety and to maintain the repression of the wish. This insight is fostered by interpreting or hypothesizing a link between the patient's current pattern of behavior, feelings and cognitions with this unconscious process. In addition, therapists hypothesize a link between the patient's patterns of past relationships, current relationships, and his/her interactions within the group.

The group relevant behaviors required for success in STG irvolve an ability to work with interpretations. Working with interpretations requires that the patient has an "ability to see relationships among 
thoughts, feelings, and actions, with the goal of learning the meanings and causes of his experiences and behavior" (Appelbaum, 1973, p.36). While this definition identifies an ability that is requisite for working with interpretations, it was intended by Appelbaum as a definition of "psychological mindedness." Hence, psychological mindedness represents a patient dimension which is conceptually related to relevant STG behavior.

A review of the psychotherapy literature reveals much consensus regarding the relevance of this patient dimension for working within all forms of psychoanalytically oriented therapy. The outcome research Iiterature, however, reveals very few studies that have attempted to operationalize psychological mindedness and imvestigate its relationship with outcome. Investigators associated with the Psychotherapy Research Project of the Menninger Foundation attempted to measure this variable (Kermberg, Burstein, Coyne, Appelbarm, Horwitz, \& Voth, 1972j. Ratings of psychological mindedness were inferred from evaluators' appraisal of components they assumed to influence thinking psychologically. These components were ideational richness, reflectiveness, and control over emotions and affects. These appraisals were basad on patients' responses to a complex battery of projective and nomprojective tests (Appelbaum, 1977). There were no significant relationships between ratings of psychological mindedness and outcome. The problems associated with theix operdtional definition of psychological mindedness include its lack of objective criteria, its indirectness, and the fact that they defined a psychological concept in terms of other psycholrgical concepts rather than overt, quantifiable 
behavior.

Investigators associated with the Penn Psychotherapy Project utilized more abjective measures of patient predictor variables (Iuborsky et al., 1980). They did not, however, specifica' $7 y$ assess psychological mindedness. It was incorporated into other variables. These other variables were: inventory of social and psychological functioning, and attractiveness as a patient (a variable which included an assessment of the patient's insight). Neither variable was significantly related to outcome. Problems associated with this operational definition also include its lack of specificity and its indirectness.

The evidence for a significant relationship between psychological mindedness and therapy outcome is more substantial in investigations of group therapy than indivicual therapy. Abramowitz and Abramowitz (1974) investigated the relationship between outcome in groups and pretherapy individual assessments of insight. Based on their scores on the Insight Test (Tolor and Reznikoff, 1960), twenty-six college students were assigned to homogeneous groups (two groups composed of those who obtained high scores and two groups composed of those who obtained low scores). Each type of group attended either insight-oriented therapy or supportive therapy for ten sessions (twice weekly with sessions lasting 90 minutes). Hence, the type of composition was crossed with the type of treatment with one group in each of the four treatment conditions. Their results indicated that the highly insightful patients improved more on measures of psychosocial functioning when they experienced insight-oriented therapy 
as opposed to supportive therapy. The highly insightful patients did no better than their minimally insightful counterparts in the supportive treatment condition. These results are promising with respect to specifying the match between patient and therapy approach. Unfortunately, the methodological weaknesses necessitate that this study be viewed as exploratory rather than definitive. These weaknesses include the small number of patients and groups in each condition, the use of the senior investigator as the therapist in the study and the use of a college student population.

In the previously described comparative outcome study reported by Piper et al. (1984), psychological mindedness did correlate with the outcome of patients receiving short-term group therapy. The psychological mindedness scores of patients who particularly benefited from STG were significantly higher than patients evidencing relatively less improvement. In that study, psychological mindedness was one of fifteen variables that were rated by the therapist based on his/her interview behavior. Hence, the variable was measured globally and in conjunction with a number of other variables.

In summary, the relevance of the variable psychological mindedness to the objec:ives and requirements of STG is evident. Past attempts at operationalizing the variable have relied on indirect or nonspecific sources of assessment. A more objective, direct measure of psychological mindedness which is based on clearly defined behavioral referents would represent the development of a selection criterion that may predict work and outcome in short-term psychoanalytically oriented group therapy. 
It should be noted that developing a measure of patient suitability that is based on a pre-therapy dyadic interview does not reflect trends in the group psychotherapy research literature. Rather, it is group assessments that have been found to be better predictors of subsequent group behavior (Goldstein, Heller, \& Sechrest, 1966; Piper \& Marrache, 1981). It is probably not surprising, therefore, that the group research literature has recommended a trend away from dyadic assessment and towards the assessments of pretherapy and early therapy group behavior as selection or suitability variables. Despite the predictive ability of pretherapy group behavior on subsequent therapy group behavior, there are several clinical disadvantages inherent in this procecture. Selecting patients based on their pretherapy group behavior necessitates that the candidates have already been deemed suitable, or at least worthy of consideration, for group therapy before the referral to group assessment was made. It is this preliminary decision, made no doubt by a screening mental health professional that remains the relevant clinical issue in need of researching. In addition, clinicians have voiced concern over feelings of rejection on the patient's part, were he/she to fail the group assesment. The practical demands of utilizing pretherapy group assessments as a selection variable also tend to make the process unpopular to clinicians. These demands include recruiting and training assessment leaders (who are not the grouns' therapists) and rapidly rating the pretherapy sessions in order not to delay the onset of therapy.

Perhaps for these clinical reasons, these pretherapy assessment groups are typically utilized to prepare patients for the group 
experience, rather than for selection purposes. These preparation sessions are referred to as pretraining sessions in the literature (e.g., Piper, Debbane, Garant, and Bienvenu, 1979). Research evidence indicates that these sessions can improve attendance and dropout rates in therapy groups (Budman, Clifford, Bader, \& Bader, 1981; Piper, Debbane, Biervenu \& Garant, 1982). Budman advocated the use of a three-hour preparation workshop with his young adult short-term groups (Budman et al., 1981a). However, there are ciinical disadvantages associated with this method of preparing STG candidates. These disadvantages include the therapist's difficulty in joining a group that has been prepared by another clinician since they may have been together for as many as four sessions. In the case where the group therapist conducts the pretraining sessions, the disadvantage involves the patients' reactions to the inevitable shift in the therapist's role (directive to interpretive). In addition, spending the equivalent of a quarter of the therapy groun's time in preparation, may undermine the efficiency of short-tem group therapy. Hence, there are practical considerations to the development of a measure of psychological mindedness. These considerations include the ease and speed of its administration and the ease with which it could be incorporated into typical patient selection and preparatory procedures. The next two sections summarize the rationale for the present study and present the major hypotheses. 


\section{The Present Study}

It is apparant from the literature review, that there is considerable controversy and excitement concerning short-term group therapy utilizing the psychoanalytically oriented approach. The excitement reflects pragmatic and theoretical developments in the mental health field. The controversy centres around fundamental issues, particularly the question of efficacy. The primary consideration of the present study was to conduct a clinical-trial investigation of STG. The objective of this form of therapy is to help patients solve their presenting problems by achieving insight into how their difficulties are related to unresolved intrapsychic conflicts and by initiating a process of working through that will continue beyond the treatment sessions. The therapist used an interpretive, here-and-now approach that focuses on unconscious conficts. Treatment consisted of twelve weekly sessions of 90 minutes duration. Treatment was offered to half the patients inmodiately while the others waited twelve weeks before beginning treatment. This waiting period constitued the no-treatment control condition. It was equivalent in length to the treatment phase. There was a six-month follow-up assessment period for all treated patients.

Consistent with recommendations offered in the literature, patients participating in the present study represented a population whose problems might be particularly conducive to work and resolution in STG. The patient population chosen were adult men and women who had lost a significant other in the recent past. Psychoanalytic theory 
assumes that the difficulties experienced by these patients are related to the exacerbation of unconscious conflicts, for example independence versus dependence or autonomy versus intimacy. While such conflicts are experienced by everyone and a partial resolution has likely been previously achieved by these patients, it was hypothesized that the debilitiating effect of the loss followed a re-intensification of the conflicts. It was believed that STG could offer loss patients the opportunity to explore and negotiate a new resolution to their conflicts. By experiencing the inevitable loss of the group, unresolved conflicts and feelings associated with each patient's idiosyncratic loss could be re-experienced and addressed in the here-and-now group situation. Consistent with Mann's (1973) model of time-limited individual therapy, by understanding their reaction to the loss of the group, the patients may bergin to work through unresolved conflicts that are associated with their previous losses.

Given the complexity of the outcome question in terms of the contribution of patient variables and therapy process variables in deternining treatment cutcome, these other variables were also investigated by the present study. specifically, the uncertainty regarding the feasibility of carrying-out psychoanalytic work on an on-going basis in a short-term group was addressed. The quality and quantity of psychoanalytic work evidenced by patients and therapists during the therapy sessions were monitored utilizing the Psychodynamic Work and Object Rating System (FwORS; Piper \& MoCallum, 1988). The FWORS defines work as an attempt by a group member to understand the problems of one or more members of the group, or the group as a whole 
in terms of conflict among dynamic components (wishes, fears, defensive processes). By monitoring the level of psychodynamic work from session to session, the incidence of and thereby feasibility of conducting psychoanalytic work could be explored. These quantitative indices of work could subsequently be correlated with outcome. Hence, these analyses would address the relationship between therapy process and outcome in the groups. Theoretically, patients who evidence higher: levels of psychodynamic work when participating in a psychoanalytically oriented therapy, are assumed to evidence better outcome scores relative to patients who evidence low levels of psychodynamic work.

The Rwors differentiates cwo levels of work and two levels of non-work. In addition to the level of work, the pwors also monitors the focus of the work, in terms of which member(s), the work involves. Finally, the Fwors monitors the degree to which group members (including the therapist) link patterns of interpersonal relationships. A comprehensive description of the Pwors is presented in the Method section.

The third consideration of the present study was the potential usefulness of the patient dimension, psychological mindedness for predicting the process and outcome of short-term psychoanalytically oriented group therapy. To merit usefulness as a selection criterion, a direct relationship between levels of psychological mindedness and outcome would have to be demonstrated. In terms of process, it was assumed that patients considered highly psychologically minded, would evidence high levels of psychodynamic work. conversely, patients considered marginally psychologically minded, would evidence low levels 
of psychodymamic work.

In this irvestigation, psychological mindedness is defined as the ability to identify dynamic (intrapsychic) components and to relate them to a person's difficulties. In onder to assess the patient's level of psychological mindedness, an assessment device was constructed. The assessment device is called the Psychological Mindedness Assessment Procedure (PMAP; McCallum \& Piper, 1988). The test stimulus is a videotape of simulated patient-therapist interactions. The interactions are portrayed by an actress and an actor who follow a script which was designed to reflect various aspects of therapy process. The rating scale identifies nine levels of psychological mindedness. Each level reflects a different concept relevant to psychoanalytic theory. A manual outlining specific rating criteria for each level has been developed. Hence, actvantages to this operationalization of the concept include its basis in direct and behavioral referents. Its clinical advantages include the ease and speed of its administration and the ease with which it can be incorporated into typical patient selection and preparatory procectures. In addition, the conceptual relevance of the dimension to clinicians may make it popular with them. A possible disadvantage of the PMAP relates to the standardization of the stimulus. It is quite possible that patients who display high levels of psychological mindedness with respect to the actress-patient's problem, do not generalize this ability to their own problems. Hence, this measure may not predict therapy process or outcome on the level of the individual patients. However, given that groum therapy involves the feedback and 
interaction between patients, the PMAP may still predict the work level of the group. A comprehensive description of the measure is presented in the Method section.

In sumary, the present study investigated the efficacy of and patient suitability for short-term psychoanalytically oriented group therapy with patients who experience difficulties after a person loss. There was an immediate treatment condition and a delayed treatment condition. There was also a follow-up assessment. Patients were matched on the suitability variable and randomly assigned to treatment condition. Each grour consisted of equivalent mubers of patients scoring highly or marginally suitable in terms of psychological mindedness.

\section{Hypotheses}

In the present study eight major hypotheses were formulated.

\section{Hypothesis 1}

Greater benefits, in terms of outcome indices, will be reported by the treated patients relative to the wait list control patients.

The first hypothesis focuses on the efficacy of short-term psychoanalytically oriented group therapy. This hypothesis is based on previous reports in the clinical and research literature which support the efficacy of STG. 
Hypothesis 2

Greater benefits, in terms of outcome indices, will be reported by the highly suitable patients, relative to the marginally suitable patients.

The second hypothesis focuses on the patient suitability variable, psychological mindedness, as a curative factor. Psychological mindedness may exert an independent effert on outcome. Hence, patients who are psychologically minded may resolve their difficulties, whether treated or not. This hypothesis follows from the conceptual definition of the variable. The highly psychologically minded patient, by definition, is able to explore connections between overt behavior, affects and cognitions, including symptomatology, and unconscious intrapsychic conflicts. Hence, the results will indicate an independent effect for suitability.

\section{Hypothesis 3}

There will be an additive effect between treatment and suitability. The consequence of this additive effect will result in the treated highly suitable patients reporting the best outcomes, the untreated marginally suitable patients reporting the worst outcomes, and the remaining two groups of patients (i.e. the treated marginally suitable patients and the untreated highly suitable patients) reporting outcomes in between.

The third hypothesis predicts that the effects of therapy and psychological mindedness will cambine such that highly psychologically minded patients in the immediate treatment condition will benefit from 
both independent variables and evidence the best outcomes. The outcomes of marginally psychologically minded patients in the immediate treatment condition or those of highly psychologically minded patients in the delayed treatment condition will reflect the effects of only one of the independent variables (i.e. treatment or suitablity, respectively). Hence the outcomes of these two groups of patients will be less impressive than those of the inmediately treated highly psychologically minded patients but more impressive than those of the marginally psychologically minded patients in the delayed treatment condition. The outcomes of this latter group of patients will reflect the beneficial effects of neither the treatment nor the suitability variable.

\section{Hypothesis 4}

Treated highly suitable patients will evidence better outcomes than treated marginally suitable patients.

The fourth hypothesis addresses the utility of psychological mindedness as a selection criterion. This hypothesis is based on the conceptual definition of the patient variable. By definition, the highly psychologically minded patient considers similar bases to problems as the therapists. Hence, therapist interpretations will have a more pronounced impact on the highly psychologically minded patients compared to the marginally psychologically minded patients.

\section{Hypothesis 5}

Untreated highly suitable patients will evidence better outcomes 
than untreated marginally suitable patients.

This hypothesis addresses the utility of psychological mindedness as a prognostic factor. While waiting for therapy, the highly psychologically minded patient has more ability than the marginally psychologically minded patient to initiate a process of self-examination that may resolve some of his/her difficulties.

\section{Hypothesis 6}

Highly suitable patients will evidence higher levels of psychodynamic work during the sessions, in terms of process ratings, than marginal suitable patients.

The issue of therapy process is addressed by the sixth hypothesis. Given the parallels between the operational definitions of psychological mindedness and psychodynamic work, the highly psychologically minded patients' ability to identify the actress-patient's conflictual intrapsychic components on the PMAP will be similar to their ability to identify group members' conflictual intrapsychic components.

\section{Hypothesis 7}

Levels of psychodynamic work will be positively associated with therapy outcome.

This hypothesis follows from theory concerning curative factors in psychoanalytically oriented therapy. The objettive of this approach is to help patients solve their presenting problems by achieving insight into how their difficulties are related to unresolved intrapsychic 
conflicts. Patients who engage in greater psychodynamic work will, by definition, evidence greater insight into the association between intrapsychic conflicts and their difficulties and will, therefore, benefit more from this approach.

\section{Hypothesis 8}

The benefits evidenced by all treated patients will be maintained at the six-month follow-up period, as indicated by the follow-up outcome indices.

The eighth hypothesis is based on the notion that STG will thoroughly address the issue of loss such that the process of working through, initiated in the group, can be continued beyond the treatment sessions. In addition, it is assumed that by thoroughly exploring the issue of loss, the patients will benefit by obtaining an important example or model for understanding concurrent and subsequent conflicts in their lives. Hence, the work of therapy will continue after the formal therapy has been completed. 


\section{Yethod}

\section{Design}

An overview of the design is presented in Table 1. The study was designed to investigate the effectiveness of short-term, psychoanalytically oriented group psychotherapy (STG) witn psychiatric outpatients who experience difficulties following the loss of a person. The first independent variable, treatment, represented a fixed-effect, crossed variable with two levels: presence versus absence of STG. The study was also designed to provide information about the suitability of patients for STG. This irvolved an investigation of the personality characteristic psychological mindedness (PM) in terms of its potential value as a selection criterion for this type of therapy and as a prognostic variable for this type of patient. Patient suitability formed a second, fixed-effect, crossed independent variable with two levels: highly versus marginally in terms of psychological mindedness. Each group was to be composed of four patients who were highly psychologically minded and four patients who were marginally psychologically minded. The three types of time periods were treatment, waiting-list control, and follow-up. The length of treatment and of the delay period was an equivalent twelve weeks. The follow-up period was six months.

Eight therapy groups were composed over a fourteen month period; four in the immediate treatment condition and four in the delayed treatment condition. There were two therapists in the study. Each 


\section{Table 1}

Overview of The Design

(A)

Treatment

\begin{tabular}{|c|c|c|c|c|c|c|c|c|c|c|c|c|}
\hline 1 & (B) & 1 & & Inmec & diate & 1 & & \multicolumn{4}{|c|}{ |Delayed Treatment | Follow-up | } \\
\hline I Therapist & 1 Suitability & & & e-Mont & th $\mathrm{P}$ & $1]$ & e-Mont & th Per & $1 \mathrm{~T}$ & hree $M$ & ionth Period: & |Six-Month Period| \\
\hline 1 & 1 & 1 & (D) & $\underline{1}$ & $\underline{5}$ & 1 & $\underline{2}$ & $\underline{6}$ & 1 & $\underline{2}$ & $\underline{6}$ & $\begin{array}{llll}1 & 5 & 2 & 6\end{array} \mid$ \\
\hline |Therapist 1 & |Highly suitable & 1 & & $|4 \mathrm{H}|$ & $|4 \mathrm{H}|$ & 1 & $|4 \mathrm{H}|$ & $|4 \mathrm{H}|$ & 1 & $|4 \mathrm{H}|$ & $|4 \mathrm{H}|$ & || $4 \mathrm{H}|| 4 \mathrm{H}|| 4 \mathrm{H}|| 4 \mathrm{H}||$ \\
\hline 1 & |Marginally Suitable & & & $|\underline{4 M}|$ & $|\underline{4} \mathbf{M}|$ & 1 & $|\underline{4 M}|$ & $|4 M|$ & 1 & $|\underline{4 M}|$ & $|\underline{4 M}|$ & ||$\underline{4 M}|| \underline{4 M}|| \underline{4 M}|| \underline{4 M}||$ \\
\hline 1 & $\perp$ & 1 & & & & $\perp$ & & & 1 & & & 1 \\
\hline 1 & 1 & 1 & & 3 & 7 & 1 & 4 & 8 & 1 & 4 & 8 & $\left|\begin{array}{llll}3 & 7 & 4 & 8\end{array}\right|$ \\
\hline Therapist 2 & |Highly Suitable & 1 & & $|4 \mathrm{H}|$ & $|4 \mathrm{H}|$ & 1 & $|4 \mathrm{H}|$ & $|4 \mathrm{H}|$ & 1 & $|4 \mathrm{H}|$ & $|4 \mathrm{H}|$ & || $4 \mathrm{H}|| 4 \mathrm{H}|| 4 \mathrm{H}|| 4 \mathrm{H}||$ \\
\hline 1 & |Marginally Suitable & & & $|\underline{M M}|$ & $|\underline{4 M}|$ & i & $|\underline{|M|}|$ & $|\underline{4 M}|$ & 1 & $|\underline{4 M}|$ & $|\underline{4 M}|$ & ||$\underline{4 M}|| \underline{4 M}|| \underline{4 M}|| \underline{4 M} \mid$ \\
\hline & 1 & 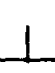 & & & & & & & 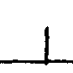 & & & \\
\hline
\end{tabular}
(A) Treatment
(B) Suitability: 4H - four patients scoring high in psychological mindedness - highly suitable
$4 \mathrm{M}$ - four patients scoring marginally in psychological mindedness - marginally suitable
(C) Therapist
(D) Groups 
therapist led two groups in each treatment condition. Therapist represented, therefore, a random-effect, crossed independent variable with two levels: therapist one and therapist two. The groups represented a partially nested, random-effect variable with two groups being nested within each cell of the treatment versus therapist variables. As each group was composed of both highly and marginally suitable patients, the groups were not nested within the suitability variable. Fairs of patients were matched on level of PM, sex, and age with one of the pair being randomly assigned to either the immediate treatment group or the delayed treatment group. Hence, the method of patient allocation was a combination of matching and randon assignonent.

In sumary, the study involved a mixed model design with three completely crossed independent variables (treatment, PM, therapist) and one partially nested independent variable (groups). Two of the crossed independent variables, treatment (presence versus absence of STG) and suitability (highly versus marginally psychologically minded) were fixed-effect variables. The other crossed independent variable, therapist (therapist one versus therapist two) was a random-effect variable. The therapy groups represented a partially nested, random-effect variable with two therapy groups being nested within each cell of the treatment versus therapist variables but not the suitability variable.

The dependent variables in this study included outcome measures, and therapy process measures. Main and interaction effects of treatment and suitability were investigated utilizing a series of univariate and multivariate statistics. Multiple comparison tests were 
used where appropriate. Treatment effects were examined by comparing the outcomes of patients in the immediate treatment condition versus patients in the wait group. Treatment effects were also examined by comparing the outcomes of patients in the delayed treatment condition over the wait period versus over the treatment phase. To determine the relationships between pairs of variables, comparisons across conditions were made using correlational techniques.

\section{Patients}

Seventy-nine adult outpatients (53 female and 26 male) of the walk-in Clinic of the Division of External Psychiatric Services, University of Alberta Hospitals (Edmonton, Alberta) participated in the study. These patients were referred to the Walk-in Clinic by general practitioners $(44 \%)$, friends $(24 \%)$, private psychiatrists (13\%), social agencies $(12 \%)$ or themselves $(6 \%)$. Prior to the beginning of the study, a notice had been circulated to various referral sources in the city of Edmonton informing them of the structure of the groups and the population they were intended to address (see Appendix A). AII patients had lost a significant person in the recent past, e.g., a spouse, partner, parent, family member or friend through death, separation, or civorce. They were not adapting well to the change and wished to examine the reasons with others who had had a similar experience. Since the therapy was not crisis intervention the patients were beyond the initial period of shock and mourning. Thus, for most patients the loss had not occurred during the last three months. 
Although the patients were no longer experiencing the immodiate effects of the crisis, they were still considerably affected by the loss as reflected in their inability to resume a satisfying and productive life. Depressive symptomatology, social isolation, and loneliness were typical presenting complaints.

The average length of time since the loss was seven years (range: 3 months - 20 years). The average age of the patient when he/she experienced the loss was 30.3 years (range: 5 years - 55 years). With respect to the type of loss, 33 percent reported loss(es) through death only, one-fifth (20\%) was through separation or divorce only, and almost one-half (47\%) had experienced both types of losses. For those reporting loss(es) through death, 54 percent had lost one parent and one-third had lost both parents. With respect to the number of losses identified, 35 percent reported a single loss, 26 percent reported two losses, and 39 percent reported having experienced multiple losses.

In terms of the piagnostic and statistical Manual of Mental Disorders (DSM-III; American Psychiatric Association, 1980), on admission to the clinic, 53 percent of the patients received an axis I diagnosis of affective disorder: 46 percent major depression, 6 percent dysthymic disorder, and I percent cyclothymic disorder. Twenty-three percent of the patients were diagnosed with adjustment disorder. other axis I diagnoses included generalized anxiety disonder, obsessivecompulsive disorder and conditions not attributable to a mental disorder such as marital problem or work inhibition. Nineteen percent were assessed as also warranting an axis II diagnosis of personality disorder. The most common axis II diagnosis was dependent personality 
disorder (66\%). Other personality disorders diagnosed on admission included compulsive, borderline, narcissistic and atypical. Patients manifesting problems of suicidal intent, psychosis, addiction, sexual deviation, sociopathic behavior or who were currently involved in another form of psychotherapy were excluded from the study. Seventy percent of the sample had had previous contact with a mental health professional. Few patients, however, had actually engagen in psychotherapy $(\underline{n}=5)$. In terms of medication, 49 percent of the patients had been prescribed antidepressant or anxiolytic medication.

The patients ranged in age from 18 to 65 years with a mean age of 35.7 years. The majority (89\%) had at least a high school level of ecucation. of these, almost one-third (30\%) had attended or were attending university while more than a third (37\%) had attended or were attending a technical college. At the time of the study, over two-thirds of the patients worked outside of the home (42\% full-time employment, $16 \%$ part-time employment, $10 \%$ students), while the remainder were either unemployed (18\%), housewives/househusbands (9\%) or retired (4\%). Approximately three-quarters of the patients did not live with a partner, being either single, separated, divorced or widowed (28\%, 20\%, 19\%, and 10\%, respectively). Sixteen percent were married and 6 percent were living common-law.

III. Therapists

Two staff therapists each concucted four therapy groups; two in each of the immediate treatment and delayed treatment condition. They 
were a thirty-four year old male psychologist and a thirty year old female social worker. Both were experienced in group and individual forms of dymamically-oriented psychotherapy (eight and four years, respectively). The practice of outpatient psychotherapy represented a major portion of their professional work.

A technical outline and manual was developed for STG (see Appendix B). It included a summary of the psychodymamic approach, the therapeutic goals, the structure of the groups and the implications that this structure might have for therapist activity. In addition, the stages of the group and the unconscious conflicts that were anticipated to emerge in the groups were described with examples of how the therapist might address and interpret thenn. A series of weekly meetings among the group therapists, the director of the research unit, the principal investigator and other therapists in the clinic was held. At these meetings, conceptual and technical aspects of the therapy were discussed and audiotaped sessions from each therapist's groups were reviewed in reference to technical adherence to the manual. The meetings began four months prior to the study and continued throughout. Audiotaped sessions from a pilot STG conducted by the primary researcher served as training material for the therapists. On-going groums were viewed from behind a one-way mirror, allowing for additional on-going supervision for the therapists. The therapists were blind to the hypotheses of the study and the suitability level of the patients. They were informed as to the treatment condition of each group as this would have been impossible to keep hidden. The therapists agreed to complete post-sessional 
questionnaires and outcome measures.

IV. Initial Clinical Interview (Intake)

The intake procedure involved several activities and typically took one-half day to complete. When a patient presented at the clinic, he/she was first asked to complete several forms incuiring about demographic information and his/ner reasons for seeking the clinic's services. Symptom checklists were also included in this package. Next, a staff therapist conducted a psychiatric assessment interview to determine a provisional diagnostic and etiological understanding of the presenting complaint(s) with the goal of formulating an appropriate treatment plan. The treatment plan was then discussed and finalized in the presence of a supervising psychiatrist. If the patient was thought to be a potential candidate for STG, the therapist made subsequent appointments to obtain his/her informed consent, to prepare him/her for the group, and to complete the referral procedure.

There were fifteen intake therapists, thirteen women and two men. Nine of the therapists held a Master's degree in social work, three held a Master's degree in psychology, two held a Bachelor level degree, one in nursing, the other in cocupational therapy and there was one Ph.D. candidate in clinical psychology. All therapists were supervised by one of seven male staff psychiatrists (four of whom were also psychoanalysts). Therapists and their supervisors were familiar with the selection criteria and format of the groups through inservice 
research seminars presented prior to the study. They also received informational memoranda (see Appendix C) and informal reminders throughout the study. In addition, many of the intake therapists participated in the STG training seminar.

Determining that a patient was appropriate for STG involved both clinical and research considerations. The clinical considerations primarily irrolved deciding whether the patient met the selection criteria (e.g. adult man or woman who had experienced a significant person loss) and was willing to be considered for STG. To help the patient make an informed decision, he/she was told that the therapy group would consist of seven or eight members and would meet weekly for 90-minute sessions over a twelve-week span. With respect to the format of the groups, he/she was told that while the groups would be relatively unstructured, the task was to explore the feelings and thoughts associated with the loss. A form summarizing the group's ground rules was presented and discussed with the patient (see Appendix D). The ground rules included the importance of commitment, confidentiality and suggestions as to how to comport him/herself in the group (e.g. to be as honest as possible conceming thoughts and feelings experienced in the group). When the clinical considerations had been satisfied, the intake therapist proceeded to discuss the research considerations.

The research considerations included the patient's willingness to cooperate with the evaluation prococtures. In this respect, the patient was told that all sessions would be tape-recorded, that a sne-way mirror facility would be used, that he/she would be asked to complete 
questionnaires throughout the therapy and that he/she would be invited to meet with members of the Research and Evaluation Unit before and after therapy and six months thereafter. The possibility of having to wait for treatment was discussed in detail. Any patient who was uncomfortable with this aspect of STG was offered an alternative treatment plan. With both clinical and research considerations being satisfied, the patient was presented with the informed consent form (see Appendix E).

The first page of the informed consent form sumarized the information concerming the therapy and the evaluation procedures. A copy of this information page together with a copy of the group's ground rules were given to the patient to keep. On the second page of the informed consent form, there was a line for the patient's signature which the intake therapist witnessed and dated. The therapist completed a STG referral form which asked him/her to detail the patient's identified loss(es). The referral form also inquired as to the therapist's reasons for referring the patient and any reservations he/she held concerning the appropriateness of the referral (see Appendix F). The signed informed consent form and the completed referral form were then forwarded to the clinic's group coordinator. The group coordinator discussed with the principal imestigator, the appropriateness of each referred patient before forwarding the referral and consent forms to the research unit.

on ocasion, the intake therapist's preparation of the patient for the group consisted of a preliminary treatment. A patient who was still experiencing the initial period of shock and mouming typically 
was offered crisis intervention and then reassessed for STG. Similarly, a patient who initially presented in a severely depressed state was typically offered a psychopharmacological intervention and then reassessed for STG. once a patient was acceptod into the STG program, however, the intake therapist's involvement was nestricted to medication monitoring and sporadic supportive contacts during the delay period as initiatod by the patient. If a patient contacted his/her intake therapist during the treatment phase of the study he/she was encouraged to discuss the concerms in the group with the STG therapist. In actuality, patients rarely contacted their intake therapist during the delay or treatment periods.

\section{Initial Research Interview}

Upon recelpt of the referral and consent forms fram the group coordinator, an independent assessor contacted the patient. An appointment for the initial research interview was arranged. This interview involved several activities and typically took three hours to complete. These activities included detemining the patient's personalized target objectives for therapy, acministering the Psychological Mindedness Assessment Procedure (PMAP), conducting a semi-structured interview to assess pretherapy levels of functioning in several areas of the patient's life and administering other measures of the pretherapy outoome battery. These activities comprised, therefore, two main tasks: the assessment of patient suitability and the administration of the outcome battery. In an attempt to keep the 
outcome assessor blind to the suitability of the patient, the tasks were divided among two independent assessors.

The initial research interview was conducted, therefore, in two parts by two members of the Research and Evaluation Unit. The independent assessors were engaged as research assistants. One of them held degrees in mursing and research technology with supplemental credits in computer and statistics courses. The other research assistant held a Bachelor's degree in psychology. Both had received extensive training in acministering the PMAP and the outcome battery. In addition, the procedure for conducting the research interviews was standardized (see Appendix G).

When a patient presented for his first research interview, he/she was asked to complete a sheet detailing the reasons for seeking therapy. When completed, one of the independent assessors, the suitability assessor, conducted the PMAP. The PMAP was presented to the patient as a way of determining his/her perception of therapy. After the PMAP was completed the other independent assessnr, the outcome assessor, conducted the semi-structured assessment interview. The patient's responses to both the PMAP and the outcome interview were tape-recorded for subsequent reliability determinations. At the end of the interview, the outcome assessor discussed with the patient, his/her reasons for seeking therapy. Using the patient's previously completed list of reasons as a guide, thoy developed a set of clear and concisely stated personalized target objectives. These target objectives would later be rated by the patient, the outcome assessor and the group therapist. 
After the interview, the patient was told that he/she would be contacted within a few weeks concerning the starting date of his/her therapy group (i.e. relatively immediately or after a twelve-week delay). He/she was then given a questionnaire packet and asked to complete the forms in a separate office and return them to the receptionist. This packet contained the remainder of the outcome battery.

\section{Group Composition}

In composing the groups, the design called for equivalent numbers of patients scoring high and marginal on the Psychological Mindedness Assessment Procedure (PMAP) in each group. Attention was also to be given to balancing the groups according to the average age and the sex ratio. The composition process began, therefore, by accumulating enough referrals of patients who spanned the range of Psychological Mindedness (PM). Patients could then be paired according to their PM score, sex, age and, where possible, the type of loss experienced. It should be mentioned at this point that due to the nature of referred patients, there were deviations in the procedure of composing the groups.

Fewer referred patients scored marginal than high on the PMAP. This suitability ratio imbalance represented a critical unforseen obstacle to implementing the proposed design of the study. Insistence on the accumulation of equivalent numbers of marginal and high PM patients would have meant imposing a protracted wait period on all 
patients. In other words, there would have been a delay between the initial research interview and patient assigrment while awaiting the referral of additional marginal PM patients. Clinical and ethical considerations argued against the imposition of an additional wait period, especially for those patients who would subsequently be assigned to the delayed treatment condition. In practical terms, there was the concern that patients would become frustrated with a protracted and ambiguous pretherapy wait and abandon the project. Further, a protracted delay between the initial research assessment and patient assigrment could have meant significant variability in the length of the wait period. Such variability could have resulted in an inmediate treatment patient actually waiting longer for treatment than a delayed treatment patient depending on when the referral was received. This variability would have confounded the results of comparisons between the treatment versus control contitions. In sumnary, adherence to one aspect of the design (balanced ratio of level of PM in each group) threatened the implementation of the second aspect of the design (a controlled outcome study). For these aforementioned reasons, it was decided that, where necessary, the groups would be composed of fewer marginal PM patients than high PM patients.

Typically the groups were composed of two or three patients who had scored marginal on the PMAP and four or five patients who had scored high. Care was taken to ensure that the ratio of marginal to high PM patients was matched for each pair of immediate and delayed treatment groups. Overall, the groups in the immediate and delayed treatment condition were composed of fourteen marginal and nineteen high PM 
patients. Hence, the ratio of marginal to high PM patients was identical for the immediate and delay conditions.

With respect to the sex balance of the groups, there were fewer men than women referred to the project. This sex ratio imbalance was consistent with the demographics of this and other outpatient clinics (i.e. more women than men tend to seek psychotherapy). Typically the groups were composed of two or three men and four or five women. Care was taken to ensure that each pair of immediate treatment and delayed treatment groups had an equivalent ratio of men to women. Overall, the groups in the inmediats treatment condition were composed of twenty-one women and twelve men while the groups in the delayed treatment condition were composed of twenty-two women and eleven men. Hence, the ratio of men to women was similar for the immediate and delay conditions. After the two groups had been composed, one of the independent assessors contacted the patients and informed them of their group's starting date.

VII. Therapy

For patients assigned to the irmediate treatment condition, therapy began within three weeks of the initial research interview. For patients assigned to the delayed treatment condition, therapy began within two weeks of the postwait research interview, or within fifteen weeks of the initial research interview. The group therapists did not meet with the patients before therapy began. They were given a list of their group members and each patient's loss(es) as identified by the 
intake therapists. The group therapists had access to each patient's clinical record but the results of the research assessments were not available to them.

All group sessions were held at the Walk-in Clinic of Extemal Psychiatric Services. Six groups began with seven patients and two groups began with six patients. Each group was conducted by one therapist. The groups met once weekly for 90-minute sessions. The duration of the groups was limited to twelve sessions and after the second session, the membership was closed.

The conceptual orientation was psychoanalytic, that is, based on the notion that recurrent intermal conflicts whose components are largely unconscious serve to pexpetuate maladaption. Conflicts concerning the issues of intimacy versus isolation and independence versus dependence in the context of loss were common in the patient population. The technical orientation emphasized an active therapist role where interpretation and clarification were emphasized relative to support and direction. Relevant here-and-now events in the group, incluaing transference, were highlighted and examined. Patients were encouraged to contribute to the therapeutic process of other patients. The technical procedures followed those of Goldberg et al. (1983) and the therapy mamual outline (see Appendix B).

The integrity of the psychodynamic therapeutic orientation was investigated. This investigation involved process analysis ratings of the therapists' activity veilizing the Psychodynamic Work and object Rating system (FWORS). The PWORS summary indicated that the average number of therapist interventions per session was 17 . This number 
represented a participation ratio of 16 percent, relative to the total verbal productions of an average group session. Sixty-three percent of all therapist interventions were rated interpretations (statements identifying dymamic components). One-fifth of all therapist interventions involved interpretations of multiple conflictual dynamic components. These data were accepted as strong confirmation of the integrity of the therapy as being psychodynamically oriented.

\section{Follow-through Assessments}

A patient assigned to the delayed therapy group, was re-assessed between ten and twelve weeks after the initial research interview regardless of whether or not he/she intended on entering the therapy group. This second, postwait, research interview assessed changes that may have occurced during the waiting period. It was similar in structure to the first interview. The PMAP was administered by the suitability assessor. The outcome assessor then conducted the semi-structured assessment interview, assessed the severity of the target objectives, asked whether additional objectives were to be added, and investigated whether or not the patient had participated in other forms of psychotherapy or had taken medication during the wait period. Patients who had decided not to continue into the treatment phase, were given an opportunity to disiuss these reasons with the outcome assessor. Finally, he/she was given a questionnaire packet and asked to complete the forms in a separate office and return them to the receptionist. The therapist completed a brief rating form after every 
therapy session and a more comprehensive questionnaire packet after each thind of therapy.

At the end of treatment, all patients were recontacted by one of the independent assessors and asked to come in for an interview, regardless of whether or not they had actually completed the therapy. This posttreatment follow-through interview was similar in structure to the previous interview(s): the PMAP was readministered, the semi-structured assessment interview was concucted, participation in concurrent treatments was investigated, the severity of the target objectives was assessed, and a questionnaire packet was given and completed. The therapist was also given a questionnaire packet to complete.

Patients who had not completed therapy, were given an opportunity to discuss their reasons for dropping out with the outcome assessor. They were thanked for their cooperation $t$ - zughout the study and informed that any subsequent contact with the clinic should be directed to their intake therapist. Patients who had completed therapy, were reminded that they would be recontacted in six months for another, follow-up interview. Involvement with the clinic during the follow-up period was restricted to medication monitoring and sporadic supportive contacts with the intake therapist as initiated by the patient.

Six months after treatment had ended, those patients who had completed treatment vere recontacted by one of the independent assessors and asked to come in for an interview. This second posttreatment follow-through interview was identical in structure to the previous interview. At completion of the interview, the patient 
was reminded that this was the last evaluation session and was thanked for his/her cooperation throughout the study. Those patients requesting subsequent therapy were advised to recontact their intake therapist.

\section{Measures}

There were several periods of assessment in the present study. The suitability and outcome variables were assessed on three occasions for patients in the immediate treatment condition: initial, posttherapy and six-month follow-up interviews. Patients in the delayed treatment condition were assessed one additional time on these variables: following the wait period. Therapist-rated process variables were assessed after every session while therapist-rated patient variables were assessed after each thind of the therapy. Hence, a variety of measures were utilized to assess patient variables, therapy process, and outcome. Table 2 provides an overview of each measure and its time of measurement. The Appendices contain a copy of each measure utilized. The following subsections present a description of the measures, together with data concerning their psychometric properties.

\section{Patient Variables}

A. Psychological Mindedness Assessment Procedure

The Psychological Mindedness Assessment Procecture (FMAP; Mocallum \& 
Piper, 1987) was developed to meet this study's need for a direct measure of psychological mindedness that was based on clear behavioral referents. Psychological mindedness (PM) is defined as the ability to identify dynamic (intrapsychic) components and to relate them to a person's difficulties. The PAAP also assesses the degree to which a person understands psychodynamic interpretations. This second variable, called Interpretation comprehension (IC), is defined as the ability "identify the referents of therapeutic interpretations.

The PMAP (Appendix H) utilizes a videotape of two simulated patient-therapist interactions. The interactions are portrayed by actors acconding to scripts developed to reflect various components of therapeutic process. The interactions begin with an actressmatient describing a recent event in her life to her male therapist. This description includes verbalizations reflecting dynamic components (i.e. conflictual wishes and fears, defensive manoeuvres) and links between internal and external events (i.e. links between cognitions/affects and behavior). The actress-patient's account constitutes the test stimulus for assessing psychological mindedness.

The second part of the interactions imvolves an actor-therapist responding to the patient by interpreting first the dynamic components of her conflict and then the transferential aspects (i.e. her attempt to repeat with the therapist a past mode of interaction). Both types of interpretations are presented in three stages. These stages vary on the degree of ambiguity and therefore, on the degree of difficulty. The first interpretations are very ambiguous with the second and thind interpretations becoming increasingly less ambiguous or 'easier' to 
Table 2

overview of Measures and Time of Evaluation

Measure

Pre-Wait Pre-Therapy/ During Post-Therapy Follow-up Interview Post-Wait Therapy Interview Interview

Assessor-rated

Patient Variable:

PMAP

$\mathrm{x}$

$\mathrm{X}$

$\mathrm{X}$

$\mathrm{X}$

Process Variable:

FWORS

$\mathrm{x}$

outcome Variables:

Social Adjustment

Scale

X

$\mathrm{X}$

$\mathrm{x}$

$\mathrm{X}$

Target Severity $\mathrm{X}$

$\mathrm{x}$

$\mathrm{X}$

$\mathrm{X}$

Therapist-rated

Patient Variables:

Psychological

Mindedness

$x$

Likeability

$\mathrm{x}$

Response to

Interpretation

$\mathrm{X}$

Process Variables:

Patient

Participation

$\mathrm{X}$

Patient work

$\mathrm{X}$

outcome Variable:

Overall Usefulness

of Therapy

$\mathrm{X}$

Note: PMAP = Psychological Mindedness Assessment Procecure FWORS = PSychodymamic Work and Object Rating System 
Table 2 continued

Measure

Pre-Wait Pre-Therapy/ During Post-Therapy Follow-up Interview Post-Wait Therapy Interview Interview

\section{Patient-rated}

outcome variables

Target Severity

$\mathrm{x}$

$\mathrm{X}$

$\mathrm{X}$

$\mathrm{X}$

Target Change

$\mathrm{X}$

$\mathrm{X}$

$\mathrm{X}$

Impact of Events

Scale

$\mathrm{x}$

$\mathrm{X}$

$\mathrm{X}$

Interpersonal

Behavior Scale

$\mathrm{x}$

$\mathrm{X}$

$X$

Interpersonal

Dependency

Irventory

$\mathrm{x}$

$x$

$\mathrm{x}$

SCI -90

$\mathrm{X}$

$\mathrm{X}$

$\mathrm{x}$

$\mathrm{X}$

Beck Depression

Inventory

$\mathrm{X}$

$\mathrm{X}$

$\mathrm{X}$

$\mathrm{X}$

Rosenberg

Self-Esteem Scale X

$\mathrm{X}$

$\mathrm{X}$

Iife Satisfaction $x$

$\mathrm{X}$

$\mathrm{X}$

$x$

overall Usefulness

of Therapy

$\mathrm{X}$

$\mathrm{X}$

Service Evaluation

Questionnaire

$\mathrm{x}$

$\mathrm{X}$

Note: ScI-90: Symptom cheaklist - 90 items 
recognize. The actor-therapist's responses constitute the test stimulus for assessing Interpretation comprehension.

The PMAP is indivicually administered. It takes approximately thirty mimutes to complete. Both interactions are shown and fol'ow the same procedume. The first part, the patient's account, is initially shown unintemupted. After this first showing, the tape is stopped and the person being assessed is asked for his/her general impressions of "What seems to be troubling this woman?" The person's responses are audiotaped. Part one is then replayed with the person being encouraged to stop the tape at any point to elaborate or clarify his/her initial responses. Repeating the first part of the tape is an attempt to eliminate possible confounding effects due to memory differences among respondents. All responses are audiotaped and scored according to the level of PM they are judged to reflect.

The PMAP differentiates nine levels of PM (I through IX). The criteria for each level reflect basic assumptions of psychodynamic theory. The higher levels incorporate criteria from the lower levels such that each level becomes more comprehensive and complex in its focus. To obtain a high PM score, therefore, a person must reflect several of the basic assumptions held by psychodymamic therapists concerning human pathology. It is believed that a person who reflects these basic assumptions will be more amenable to the psychodynamic therapeutic approach. Conversely, to obtain a score of 0 , the person's explanation must fail to reflect any appreciation of psychodynamic theory. These explanations would be limited to the identification of external events as the cause of the patient's troubles (e.g. bad luck 
or mistreatment by others). It is believed that a person offering these types of explanations is not psychologically minded and will not be amenable to the psychodynamic therapeutic approach.

The criteria for levels I through III are based on the assumption of "psychic deteminism." Psychic determinism is the principle whereby all human functioning is assumed to result from an intermal or psychic process. Explanations of the patient's troubles that are limited to the identification of amy internal state - e.g. "she's lonely" - merit level I. Level II criteria go beyond the mere identification of an internal state and require that the explanation reflect an appreciation of the motivating aspect of this intermal state or force. For example, the statement "It's all due to her loneliness" meets level IJ criteria by implying that the loneliness is causing something ("it"). Level III demands the explicit identification of both the driving internal force and one of its results. For example, "her loneliness is making her feel depressed."

Level IV criteria are based on the assumption of the "unconscious". To score level IV, the subject must recognize that an intemal motivating force is largely out of the patient's awareness. For example, "she doesn't realize it, but she still thinks of him as her husband." Ievel $\mathrm{V}$ corresponds to the application of the concept of ambivalence and/or conflict. A basic assumption underlying the psychodymamic understanding of pathology is that internal impulses (id) come into conflict with the frustrating or nongratifying aspects of external reality or their internalized representatives (superego). This intemalized representative can be experienced as a contradicting 
affect or cognition, an anticipation of punishment or rejection, or as a prohibitive conviction based on morality or conscience. An example of a response meriting level $V$ is: "she wants to be with her husband again, but she's still angry with him."

The criteria for levels VI and VII reflect an appreciation of the assumptions that a conflict presses for discharge which in turn produces tension or disequilibrium. To score level VI, therefore, the subject must identify both the conflict and one of its results (as it pushes for discharge). For example, "she's paralyzing herself because she doesn't know whecher or not she wants to chance it with him again." A level VII is scored if the person actually labels the tension (that is resulting from the conflict) as an anxiety or fear: "despite all the hurt and anger, she still wants him back and that scares her."

Levels VIII and IX correspond to the assumption that conflictual impulses are only permitted expression in a distorted or diminished form. Hence, they are filtered through self-protecting or defensive mechanisms. Level VIII reflects the identification of a defensive process while level IX explanations emphasize that the defensive manoeurre has achieved only partial success in resolving the conflict. For exarmple, level VIII would be warranted if the person said "that's sour grapes to say she's 'really better off without him'." A level IX would be warranted if the person contimued by explaining that "she really feels that she"d be a lot happier with him."

After the level of psychological mindedness has been assessed, the second part of the tape, the therapist's interpretations, is then 
played. Part II of the PMAP is introduced by informing the patient that "[t]he therapist is going to come on and tell the patient what he thinks might be troubling her." The patient is instructed to try to understand his statements. The three interpretations of the dynamic components and the three interpretations of the transference are then played. The tape is stopped after each interpretation and the person being assessed is asked "Where is he getting that from" or "What is he driving at" or "What does he mean by that." His/her responses are audiotaped and later scored according to the level of IC they are judged to reflect.

Interpretation comprehension has three subscores. The first subscore refers to the mumber of dynamic components that are correctly understood from the therapist's dynamic interpretations. The three dynamic components interpreted are the wish, the anxiety/fear and the deferse mechanism of rationalization. The rating scale is from 0 to 3 with each dynamic being worth 1 point. A second subscore refers to the speed or ease with which the individual has understood the dynamic components. The speed is determined by the level of ambiguity at which the dynamic component was understood. This rating scale is from 0 to 9. Each component understood after the first (most ambiguous) interpretation receives 3 points, while those understood after the second interpretation receive 2 points, and those components that are only understood after the third (least ambiguous/easiest) interpretation receive $I$ point.

There is also a subscore to reflect the speed with which both aspects of the transference interpretation are understood. The two 
aspects of the transference interpretation are the past mode of interaction and the actress-patient's attempt to re-enact this with the therapist. The scale is from 0 to 6 . Each component is worth 3 points if it is understood after the first interpretation, 2 points if it is understood after the second interpretation, and 1 point if it is understood after the third interpretation.

Prior to its implementation in this study, the psychometric properties of the measure were explored in a a pilot study. Thirty adult men and women were recruited from the Montreal area to participate in a study on "the perception of psychotherapy." After completion of the videotape portion of the study, each subject was administered questionnaire measures of personality, intelligence, depression and anxiety in order to investigate the construct validity of the concept. Half of the subjects were brought back one month later to assess test-retest reliability. Two raters independently rated the (audiotaped) responses of the subjects in order to assess inter-rater reliability. The first scenario was found to have the stronger psychometric properties, regardless of the order of presentation (which was counterbalanced). The reliability and validity data which follow relate to this scenario. The results of Pearson product-moment correlation coefficients revealed that the test-retest reliability of the measure was $\underline{x}(13)=.76, \mathrm{p}<.001$ and the inter-rater reliability was $\underline{r}(13)=.81, \underline{p}<.001$. The measure was significantly related to the psychological mindedness subscale of the California Psychological

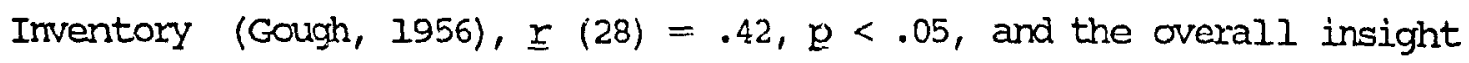
score of the Insight Scale (Tolor \& Reznikoff, 1960), $\underline{r}(28)=.50, \underline{p}<$ 
.01. It was not significantly related to the arhievement scale of the Fersonality Research Form (Jackson, 1974), the intelligence quotient of the Quick Test (Anmons \& Ammons, 1962), or the anxiety and depression subscales of the SCI-90 (Derogatis, 1977).

In sumnary, the reliability data, the positive correlations with related concepts and the independence from need achievement, intelligence and both anxiety and depression all supported the manner in which the PMAP had operationalized psychological mindedness. The PMAP promised to be an efficient, easily acministered measure that might predict amenability to psychodymamic therapy, and thcreby judged appropriate for assessing patient suitability for psychoanalytically oriented, short-term groun psychotherapy.

In this study, the patient's PM score on the first interaction determined his/her designation as highly or marginally suitable for STG. Patients who received a score of 0-5 were classified as marginally suitable while patients who received a score of 6-9 were classified as highly suitable. This cut-off point was chosen upon the basis of both conceptual and empirical considerations. Conceptually, scores lower than 5 reflect reference to an intrapsychic component as an isolated event and a score of 5 indicates only the identification of some conflict between two (or more) of these intrapsychic components. Conversely, sonres higher than 5 reflect the additional understanding of a consequence arising from the conflict between the opposing intrapsychic components. Hence, patients who, at the most, are only able to identify conflictual forces but are unable to identify their impact or consequences are seen as marginally suitable for STG. 
Conversely, patients who are 'primed' for investigating the relationship between intrapsychic conflicts and their consequences (e.g. symptoms) are seen as highly suitable for this therapeutic approach. Empirically, this area of the scale was a definite dividing region for the thirty subjects of the pilot study (12 subjects received a score of $0-5$ and 18 subjects received a score of 6-9).

In the present study, the two independent assessors were trained to administer the PMAP and rate the level of PM. The training sessions lasted approximately two-months and involved extensive practice ratings utilizing the audiotapes from the pilot study. In addition, the independent assessors, being members of the research unit, had previously attended related training sessions and discussions with the investigator concerning the basic concepts and assumptions of psychodynamic theory. Training was considered complete when acceptable inter-rater reliability coefficients had been reached.

As each assessor administered the PMAP to half of the patients, it was possible to determine the inter-rater reliability for the PMAP in this study. Tape-recordings of fifteen patients' responses to the PMAP were randomly chosen and rated by a second rater. For the first scenario, the scenario that served to ascertain patients' level of suitability, the mean Pearson product-moment correlation for two raters over the fifteen patients was $\underline{r}(13)=.90, \underline{p}<.001$. When they disagreed on the rating, they were always within one level of each other and the disagreements never affected the assignment of patients to the high versus marginal suitability categories. Hence, level of patient suitability had been reliably determined with this clinical 
population. For the second scenario, the mean Pearson product-moment correlation for the raters was $\underline{\underline{x}}(13)=.76, \underline{\mathrm{p}}<.001$.

With respect to ratings of Interpretation Comprehension, for the first scenario, the mean Pearson product-moment correlations for the variables, Number of Dynamics, and speed of Dynamics were both $\underline{r}(13)=$ $.80, \mathrm{p}<.001$. The mean correlation coefficient for the variable,

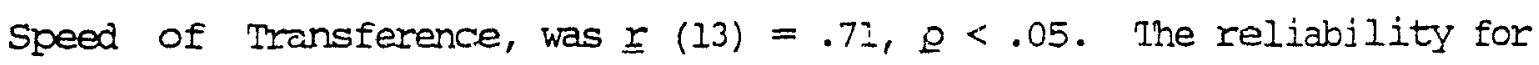
the second scenario was quite disappointing: $\subseteq(13)=.37$ for the Number of Dynamics, $\underline{I}(13)=.35$ for the Speed of Dynamics, and $\underline{I}$ (13) $=.6 i$ for Speed of Transference.

To determine whether or not the four PMAP variables represented discrete concepts, Pearson correlation coefficients were calculated for the four variables. Data were provided by the 79 patients who participated in the study. (The mean and standard deviation for each of the four PMAP variables are presented in Appendix I). As presented in Table 3, the results indicated a significant but moderate pattern of correlations between psychological mindedness and the two variables concerring dynamics. This pattern suggests that the ability to relate dynamic components to a person's difficulties (psychological mindedness) and the ability to understand therapist interpretations of conflict between dynamic components are relatively independent abilities. There may be, however, similar (coymitive) processes that underlie the two abilities. The two variables concerning dynamic components were found to be highly correlated. Since these variables are conceptually related, the strength of this correlation was expected. Finally, only minimal correlations were found between the 
Table 3

Intracorrelation coefficients for PMAP Variables

PMAP Variables

Psychological Number of Speed of Mindedness

Dynamics

Dynamics

Speed of

Transference

Psychological

Mindedness

$-$

$.34 * *$

$.25 *$

.20

Number of

Dynamics

Speed of

Dyramics

Speed of

Transference

Note: $*-p<.05$

$* *-\mathrm{p}<.01$

$\star * *-p<.001$

transference variable and the other three. This latter finding suggests that the ability to understard interpretations of transference phenomena is relatively independent of the ability to generate or to understand explanations of probiems in terms of dynamic components. Ir. summary, the PMAP is believed to assess three independent abilities although Psychological Mindedness and the ability to understand interpretations of dynamic components were found to be moderately related.

\section{B. Therapist-rated Patient Characteristics}

Following sessions four, eight, and twelve, the therapist was asked to provide ratings of each patient's level of psychological mindedness, 
likeability and response to interpretation. The rating scale for each variable ranged from poor (1) to excellent (7). These ratings were requested in order to investigate the strength of association between therapist-rated, clinical impressions and the research instruments.

\section{Therapy Process Measures}

A. Psychodynamic Work and Object Rating System

The Psychodynamic Work and object Rating System (Pwors; Piper \& Mccallum, 1987) was developed to meet this study's need for a measure that could monitor the quality and quantity of analytic work engaged in by patients and therapist(s) in group psychotherapy sessions. It is derived, in part, from the Therapist Intervention kating System (Piper, Debbane, de Carufel, \& Bierverm, 1987) and the Therapeutic Work Rating System (Connelly, Piper, \& Braha, 1985). The former process analysis system is utilized to monitor therapist interventions in individual forms of psychodynamically oriented psychotherapy. The latter process analysis system has been utilized to monitor patient work in group forms of psychodynamically oriented psychotherapy. The Pwors is an attempt to operationalize psychodynamic work in a manner that is consistent with psychodymamic theory and that can be utilized to monitor patients as well as therapists engaged in group psychotherapy.

Work is conceptualıy defined as an attempt by a group member to understand the problems of ore or more members of the group, or the group as a whole in terms of conflict among dynamic components. 
Dynamic components are internal forces in the group that are part of a conflict. This means that a dynamic component is assumed to be exerting an internal force on one or more members, or on the group as a whole and that at some level the force is oppored. Excluded from the definition of work are the mere identification or description of resultant (end) states and consideration of dynamic factors that belong to persons or situations external to the group.

There are five components in the system. Four are dynamic and one is nondynamic. The four dynamic components are wishes, reactive anxiety, defensive processes and dynamic expressions. The nondynamic component is objects. Objects refer to people - insıde or outside the group. Two aspects of abjects are monitored, the object Focus and whether or not there is object Linking. The Object Focus refers to whether the speaker is focusing on objects internal or external to the group. Internal objects include the speaker, another group member, a subgroup, or the group as a whole and are called "units of the group." External objects include family members, a specific person, another group and general classes of people. Ohject Linking refers to the identification of a shared interpersonal process between a unit of the group and two other objects. The linked objects may be internal and/or extemal to the group. The five components are used to differentiate four categories of nonwork and work which correspond to progressively higher levels of analytic work.

The pwors is consistent with psychodymamic theory in that it gives primary emphasis to understanding the role of internal (intrapsychic or intragroup) conflictual components as they are related to patients' 
problems. The importance of the group context is emphasized in that "work" includes one member's contribution to the therapy process of other members. The importance of the group context is also reflected in the system's ability to monitor conflicts involving the entire group in addition to conflicts involving dyads, subgroups, or the intrapsychic conflicts of individual members. The object Focus and Object Linking parts of the system emphasize the importance of patient-patient transference phenomena in addition to the more traditional patient-therapist transference phenomena. One use of Object Linking is to monitor the identification of maladaptive patterns of interaction being re-enacted in the here-and-now group situation.

For each patient and therapist statement, the PwORS determines the category of work, notes the object Focus and whether or not there is Object Iinking. In determining the category of work, the Fwors monitors the identification of dynamic components. Statements that do not attempt to understand the problems of a urit of the group in tems of dynamic components, are considered nonwork. There are two nonwork categories. The first nonwork category, category I, contains Externalizing statements. These statements focus on topics that do not involve a unit of the group and/or focus on objects external to the group. They fail to indicate the process in whict, a unit of the group and the external object are engaging or the impact between the two. category 1 includer statements that blame others for a unit of the group's problem(s). For example, "my father never showed any affection to anyone".

The second nomwork category, category 2, contains Descriptive 
Statements. These statements provide or request information about a unit of the group. When the statement involves an object external to the groux, the response or reaction by a unit of the group is also identified. While category 2 statements may also blame others for a unit of the group's problem(s), they differ from category I statements in that a description of the unit of the group's experience of the problem "created" by the other is at least mentioned. For example, "I probably don't like myself because my father never showed me any affection."

The work catogories contain statements that identify dynamic components of a unit of the group. Since the speaker is attempting to understand the problems of at least one group member in terms of dynamic components, the statements are considered to reflect dymamic work. The number of types of dynamic components identified in the statement differentiates the two work cacegories. The first one, category 3, contains single Dynamic component statements. These statements provide or request information about a single type of dynamic component. Category 3 includes statements that identify one aspect of a unit's intrapsychic conflict, such as a defensive process. For example, "I think you're (groum member) trying to ignore just how angry you are at your father for making you feel unacceptable and unlovable."

The second work category, zategory 4, contains Miliple Dynamic components statements. These statements provide or request information about two or more types of dynamic components, or two of the same types that are in conflict with each other. Category 4 includes statements 
that identify two aspects of an intrapsychic conflict, such as a defensive process that arises to combat reactive anxiety. For example, "I think you're (group member) trying to minimize the impact your father's death had on you because you're afraid to get in touch with how angry you are about all the other times he abandoned you." Hence, these statements indicate an appreciation of the opposing or conflictual nature of dynamic components.

In sumary, the FroRs offers a system for determining the quality and quantity of psychodynamic work evidenced by group members. Specifically, the PWORS can explore the level of work, and the focus of work in terms of which member(s) the work involves, and the degree to which group members (including the therapist) link patterns of interpersonal relationships. Hence, the EwoRs can provide information concerming the extent to which each member works within and across sessions, addresses his/her own issues or those of another unit of the group, and the extent to which maladaptive patterns of interaction are identified as being re-enacted in the here-and-now group situation. In adition to providing information concerning the therapy process of patients, the PWORS can provide the therapist. with feedback conceming his/her tochnique. The FwORS is consistent with psychodynamic theory in that primary emphasis is given to understanding the role of intrapsychic components in members' problems. The PWoRs also emphasizes the importance of the group context in that the definition of work includes one member's contribution to the therapy process of another member. An appreciation of the importance of the here-and-now group behavior is reflected in the differentiation between internal and 
external object Focus. Finally, the importance of member-member transference in addition to therapist transference is reflected in the monitoring of Object Iinking. Appendix $\mathrm{K}$ presents the FWORS system and manual.

All therapy sessions were audiotaped utilizing a SONY reel-to-reel tape recorder. A microphone was mounted on the siling of the therapy room and the reconder was placed in an observation room. The facility was equipped with a one-way mirror and a sound panel. It had been especially designed to view, monitor and record therapy sessions.

Seven of the twelve sessions $(1,2,4,6,8,10$, and 12) were chosen for process analysis. Neither the patients nor the therapist were aware of which sessions were to be chosen for rating. The objectives of this approach to selection were to obtain a large data base for all patients, especially for tr se patients who eventually dropped out, and to obtain an equivalent amount of data from each phase of therapy. It was hoped that this selection procedure would ensure a representative sampling of patient behavior, and allow an analysis of the relationships between initial therapy behavior and subsequent therapy behavior, dropping out, and/or therapy outcome.

It should be noted at this point that five of the 56 sessions could not be analyzed and had to be discarded. The discarded sessions were either inaudible (poor sound quality) or did not record due to technical (reconder malfunction) or human error. In each case, the closest session in terms of chronolgy was substituted for the discarded session. On two cccasions session 3 was substituted for the first session and session 11 replaced session 12, while for one discarded 
session 4, session 5 was substituted.

The raters listened to the first half-hour of each session for context, then rated each patient's statements churing the next 45-mimute segment. Using a similar process analysis system (the Modified Hill Interaction Matrix) with a similar patient population, Piper and Marrache (1981) determined empirically that this 45-mimute segment was highly representative of the entire session, reporting correlations which ranged from .80 to 1.00 .

Each statement was timed using a stopwatch. A statement was defined as a part of a sentence, a complete sentence, or several sentences spoken by a member of the group which was not interrupted by a statement by another member or by a silence greater than ten seconds. Total statement duration for each patient was summed for each work category (category 3 or 4 ) and were combined for all sessions rated.

The following ratios were calculated for each patient. Participation was the ratio of the patient's total statement duration over the total verbal procuction for the group. To determine the degree to which each patient engaged in psychodynamic work two ratios were calculated. Group-based Work was the proportion of a patient's work behavior (statements scoring category 3 or 4) relative to the total work behavior of the group. Self-based Work was the proportion of a patient's work behavior relative to his/her total participation. The two work ratios were recalculated utilizing statements that had scored category 4. These additional ratios were called High-level Self-based Work and High-level Group-based Work. 
Four research assistants were trained to use the Pwors. The training sessions lasted approximately four months and involved extensive practice ratings utilizing the audiotapes from a pilot therapy group previously conducted by the principal investigator. In addition, the raters, being members of the research unit, simultaneously attended related trairing sessions and discussions with the investigator concerning the basic concepts and assumptions of psychodynamic theory. Training was considered complete when acceptable inter-rater reliability coefficients had been reached.

The inter-rater reliability of the rwors was determined by comparing pairs of independent ratings of twelve sessions. The twelve sessions were randomly chosen from the eight groups. Care was taken to ensure that each phase of therapy and every combination of raters were represented in the sample. The twelve sessions provided 1572 statements (an average of 131 statements per session) for categorization. The inter-rater reliability was determined for the FWORS utilizing its four categories. The four categories were then collapsed into just two categories: work (categories 3 and 4) and Nonwork (categories 1 and 2).

Two types of inter-rater reliability were determined for the four categories of the FwORS. The first, percent perfect agreement, reflects the statement by statement category agreem nt between two raters for a therapy session. The mean percent perfect agreement between the four raters for the 12 sessions was 79 . The average percentages of perfect agreement for categories 1 through 4 were: 87, 83,67 and 66, respectively. 
The second reliability statistic calculated was the kappa coefficient (Cohen, 1960). The kappa coefficient is recommended for use with nominal scales and reflects the proportion of category agreement between two rawiss after removing the infiuence of chance-expected agreement. The mean kappa coefficient for the four raters over the twelve sessions was .69 (range $=.62-.80$ ).

These two types of inter-rater reliability were repeated utilizing the collapsed work versus Nonwork differentiation. The mean percent perfect agreement between the four raters for the twelve sessions was 89. The average percentages of perfect agreement for Work and Nonwork categories were: 85 and 90 . The mean kappa coefficient for the four raters over the twelve sessions on the Work-Norwork distinction was .75 (.78 for work and .69 for Nonwork).

This data supported the inter-rater reliability of the FWORS. When four categories were utilized, the majority of the disagreements were between the two work categories or between the two norwork categories. Thus, it was rare, that raters disagreed about the work versus nonwork distinction.

\section{B. Therapist-Rated Process Variables}

Following each session, the therapist was asked to provide an impression (expressed as a percent) of each patient's verbal contribution (participation) and the percent of each patient's participation that was corsidered "work." The therapist was asked to base his/her ratings of work on the following definution: 
Work is an attempt by the patient to understand a problem that involves the patient, other patients in the group, or the group as a whole in terns of dynamic factors. Dynamic factors include wishes, fears, defenses and any affects, behaviors or cognitions that belong to members of the group that are dynamically related to the problem. The notion of conflict is implicit if not explicitly identified. Excluded from this definition are the mere description of a problem and consideration of dynamic or nondynamic factors that belong to persons or situations external to the group.

\section{Outcome Measures}

The curcent trend in psychotherapy outcome research is to include a wide range of outcome criteria (Lambert, Shapiro, \& Bergin, 1986; Waskow \& Parloff, 1975). This trend is based on the notion that since the impact of psychotherapy is multidimensional, outcome should be monitored firom a variety of perspectives. A muber of different areas were represented in this study's outcome battery. They included interpersonal functioning, psychiatric symptomatology, self-esteem and personalized target objectives. Sources of evaluation included the patient, the therapist and the independent assessors. Consistent with current practice (e.g. Green, Gleser, Stone, \& Seifert, 1975; Mintz, Iuborsky, \& Christoph, 1979), several types of outcome indices (e.g. residual change scores, rated benefit scores) were obtained.

Seventeen of the outcome variables were assessed on three occasions: at the initial interview, at the completion of therapy, and at the six-month follow-up interview. Patients in the delayed treatment condition were assessed an additional time: following the wait period. Three outcome measures that assessed overall benefit from therapy were administered at the posttreatment and follow-up 
interviews.

\section{A. Social Adjustment Scale}

The Social Adjustment Scale (SAS; Weissman, Paykel, \& Siegel, 1972) is a semi-structured interview that utilizes 48 items to assess five areas of functioning and provide seven global ratings. The five areas assessed are work, social and leisure, extended family, marital (as spouse, parent, and member of family unit) and economic independence. The time focus of assessment is two monchs. Reliability data came from two independent studies. The degree of agreement (within 1 point) for independent raters was 95 percent. The mean Pearson correlation coefficient was .83. Validity was supported in two ways. Forty-one of the items successfully discriminated between a population of forty psychiatric patients from their normal controls. Items referring to solely single subjects (e.g. frequency of dating) failed to discriminate between the groups. The SAS was also shown to be sensitive to the impact of two months of psychotherapy as improvement in the predicted direction was measured by the SAS.

A modified version of the SAS was utilized in this study. Subscores on six areas of interpersonal functioning were obtained instead of four. The additional subscores were derived by separating the marital area of functioning into three subsections. Hence, in addition to work, social life, and parental family, the SAS, as used in this study, provided subscores in the areas of spouse, sex, and parent/child. The implementation of this modification was based on the 
belief that functioning in these three areas could be differentially affected by therapy. This modification is consistent with previous use of the scale's preciecessor, the structured and scaled Interview to Assess Maladjustment (SSIAM; Gurland, Yorkston, Stone, Frank, \& Fliess, 1972). The SSIAM provides for five subscores by providing sepairate scores for spouse and sexual life. In their use with the SSIAM, Piper and his colleagues (1984) modified the scale such that the combined spouse and parental areas were further divided into two separate assessment areas.

A second modification was made to the SAS for use in the present study. To ascertain the progressive impact of the three month wait period and the three month group therapy, the time focus of assessment was reduced to one month.

Previous work by Piper and his colleagues (1984) with the SSIAM, suggested a third modification to the SAS. These authors modified the scoring criteria to include more behavioral and frequency referents which resulted in irmproved clarity and reliability (Piper et al., 1982; 1984). In these two separate studies, the average Pearson product-moment correlation between the ratings of two independent raters were: $\underline{I}(10)=.87, \underline{p}<.001$; and $\underline{\underline{r}}(18)=.85, p<.001$. Hence, these revised scoring criteria were used in the present study with the relevant items.

Each item of the SAS was scored on an 11-point scale ranging from an absence of problems to extreme distress. The patient's score for each area was determined by averaging the ratings for that subscale. An overall score for interpersonal funct oning was obtained by 
averaging the scores of all six subscales. To determine inter-rater reliability in the present study, correlations between the two independent assessors' subscale scores for 15 patients were calculated. The mean pearson product-moment correlation for the six areas of functioning was $\underline{r}(13)=.73, p<.01$, witr. a range from $\underline{r}=$ .50 to $\underline{r}=.87$.

\section{B. Target Objectives}

A special task force commissioned of the National Institute of Mental Health presented a monograph of recommended outcome measures (Waskow \& Parloff, 1975). Within this monograph is the recommendation of including personalized target abjectives in psychotherapy outcome research. Several researchers support the recommendation of their inclusion as an important criterion of treatment efficacy (Lambert, Shapiro, \& Bergin, 1986; Sloane, Staples, Cristol, Yorkston, \& Whipple, 1975) .

In the present study, each patient was asked at the beginning of the initial research interview to specify the problems or goals that he/she most wanted help with in psychotherapy. Using this list as a guide, the cutcome assessor and the patient developed a set (between three to five) of clearly and concisely stated, nonoverlapping, personalized target objectives. The severity of the problems associated with each target objective was rated by the patient and the independent assessor.

Before beginning therapy, the patient used a 5-point rating scale 
ranging from slight (1) to extreme (5) to assess each target objective with respect to its severity during the past month and the amount of improvement expected by the end of therapy. Using the same scale, he/she was also asked to rate the importance of each target. During the interviews conducted after the termination of therapy and at the six-month follow-up, the patient was asked to use the same scale to rate the severity and importance of each target objective. Using an Il-point scale that ranged from extreme worsening (1) to extreme improvement (II), he/she was also asked to rate the type of change associated with each target objective. This rating has been called a rated benefits score (Mintz et al., 1979). A patient assigned to the delayed therapy condition was asked to rate, for each target objective, its severity, importance, expected improvement and the degree of change that may have occurred during the wait period.

At the end of therapy, the therapist used similar rating scales to rate the degree of severity and improvement associated with each patient's tanget objectives. The therapist did not provide pretherapy ratings as he/she only met the patients at the first therapy session. The outcome assessor determined the severity of target objectives by utilizing the patient's responses to a standard list of questions concerning the frequency, duration, intensity, pervasiveness, and disruptiveness of each problem associated with the target objectives. Patient responses in each dimension were rated on a scale from trivial (1) to severe (5). Giving special weight to the disruptiveness dimension, the five dimensions were averaged to produce an overall severity score (that similarly ranged from trivial (1) to severe (5)). 
The outcome assessor provided severity ratings at each assessment interview. The outcome assessor did not provide a rated benefits score, or a change score, as she often could not remember the pre-therapy severity level after the three or nine month hiatus between interviews.

Inter-rater reliability for the outcome assessors severity ratings on the first target objective from 15 patients was $\underline{r}(13)=.73 p<.01$.

\section{c. Impact of Event Scale}

The Impact of Event Scale (IES; Horowitz, Wilner, \& Alvarez, 1979) is a 15-item Likert scale which assesses the degree of subjective distress experienced in reaction to a traumatic event, such as a loss. It was included to measure the effect of this study's loss-focused therapy approach. The patient rates each item for the frequency of experience during the previous week, responding with either "not at all," "rarely," "scmetimes," or "often." The items include behavioral, affective, and cognitive aspects of distress and were based on clinical descriptions of recent episodes of distress. The scale has two subscales (as revealed by cluster analyses): the Intrusion of unwelcome aspects associated with the traumatic event, and the conscious Avoidance of other associated aspects of the event. The patient with multiple losses can complete a separate scale for each event sirce each loss is identified at the top of the copy.

The scale's split-half reliability coefficient is .86 . The internal consistency of the Intrusion subscale is .78 while that of the 
Avoidance subscale is .82 . Since the correlation between the two subscales is .42 , they are believed to reflect associated but not identical dimensions. The scale's test-retest reliability coefficient (for 1 week duration) is .89 for Intrusion, .79 for Avoidance and .87 for the total score. With respect to the validity of the IES, it was highly effective at distinguishing between patient and student populations $(p<.001)$. It has also proven itself to be sensitive to changes made by patients engaged in a treatment aimed at pest-traumatic stress disorder ( $p<.05$; mean length of treatment was 11 weeks;. In a cross-validation study, the IFS was sensitive to the stress response symdrome as evidenced in a non-clinical pooulation (Zilberg, Weiss, \& Horowitz, 1982).

\section{Interpersonal Dependency Irventory}

The Interpersonal Dependency Inventory (IDI; Hirschfeld, Kleman, Gough, Barrett, Kordhin, \& Chodoff, 1977) is a 48-item questionnaire which measures interpersonal dependency, which is defined by its authors as the need to associate closely with, interact with, and rely upon valued other people. The inventory is based on psychoanalytic, social learning and ethological theories and consists of three scales, Emotional Reliance on Another Person (ER), Lack of Social Self-confidence (SC) and Assertion of Autonony (Au). The patient is asked to rate each item on a four-point scale ranging from "not characteristic of me" to "very characteristic of me." While an overall score can be obtained, the authors suggest that a subscore for each of 
the three scales be utilized. The three scales are considered to be conceptually independent. This independence has been supported statistically. For example, the correlation between ER and Au is -.23 . With the goal of reducing the demands of self report measures on the present study's patients, the SC scale was deitted.

The IDI's preaictive validity has been supported by its ability to discriminate between normal and psychiatric populations with these results being cross-validated on two additional samples. Its construct validity has been supported by the finding that ER significantly correlated with the conceptually related variables of social desirabiity, anxiety, depression and interpersonal sensitivity. The moderate strength of these correlations, however, attest to the scale's conceptual independence from these variables. With respect to Au, the comelations were of minimal strength. With respect to its other psychometric properties, the split-half reliabilities for the relevant scales are .87 (ER) and .72 (AM).

\section{E. Interpersonal Behavior Scale}

The Interpersonal Behavior Scale (IBS; Piper, Debbane, \& Garant, 1977a) is a 30 item questionnaire which measures the present and ideal levels of interpersonal functioning. The IBS utilizes two forms which are identical in content but have different instructions: one form inquires as to the present level of functioning and the other form inquires as to the ideal level of functioning. Each form's items are divided between the patient's (present or ideal) ability to 
self-disclose, express affect and be sensitive to others. The patient is asked to rate the extent to which these behaviors are (presently or ideally) reciprocated by others. All items are rated on a -point scale ranging from "very seldom" to "very frequently". The present level of interpersonal functioning is abtained by suming the total points on the appropriate form. By determining the absolute value of the difference between the points for each item on both forms and summing them, a discrepancy score between present and ideal levels of interpersonal functioning can be abtained.

Piper et al. (1977a) found the test-retest reliabilities for 24 wait list control patients over a three month period to be $\underline{r}=.79$ for the present level of functioning scores and $\underline{r}=.74$ for the discrepancy scores. The IBS has also been found to be a valid measure of therapeutic change. Two studies have shown that treated group psychotherapy patients improve their levels of interpersonal functioning (in terms of discrepancy scores) more so than untreated wait list control patients (Piper et al., 1977a; Piper, Debbane, \& Garant, 1977b). Another study reports sirmificant improvement in group psychotherapy patients' pre to post trarapy IBS discrepancy scores (Piper et al., 1984). With respect to therapy process, the IBS present functioning scale has been shown to be significantly correlated with therapy work levels (as measured by the Hill Interaction Matrix) (Piper \& Ieonoff, 1983). 


\section{F. Rosenberg Self-Esteem Scale}

The Rosenberg Self-Esteem Scale (Rosenberg, 1979) is a 10-item Guttman scale which provides a score of global self-attitude. Theoretically, low self-esteem is believed to distinguish between pathological mourning (melancholia) and normal mouming (Freud, 1917). The assessment of self-esteem was considered, therefore, to be particularly relevant for this study's population of loss patients. The Rosenberg was chosen due co its ease of administration and impressive psychometric properties.

The patient responds to each item with either "strongly agree," "agree," "disagree," or "strongly disagree." The rating criteria yield a score from 0 to 6 with higher scores indicating lower self-esteem. The Rosenberg's coefficient of reproducibility is $92 \%$ and its coefficient of scalability is $72 \%$. These coefficients meet the criteria for Guttman scales indicating that the scale has good internal consistency, or that the items appropriately assess a unidimensional, cumulative response pattern.

The test-retest reliability (over a two-week period) ranges from .85 to .88 . The scale's construct validity is supported by its empirical relationship with conceptually relevant variables. Scores on the Rosenberg were positively related to scores of depression and anxiety but negatively related to peer sociometric ratings. Its convergent validity was supported by its moderate relationship with other measures of self-esteen (e.g. psychiatric ratings, self-Image Questionnaire). In summary, the Rosenberg represents a concise, valid 
and reliable measure of self-esteem.

G. Self-report symptom Inventory - SCI-90.

The Self-report symptom Inventory - ScL-90 (Derogatis, 1977; Derogatis \& Cleary, 1977) is a 90 item self-report symptom inventory. It consists of nine primary symptom classifications: somatization, obsessive-compulsiveness, interpersonal sensitivity, depression, anxiety, hostility, phobic anxiety, paranoid ideation and psychoticism. It was included as a comprehensive measure of psychiatric. symptomatology. In addition, the measure has been shown to ve a reliable and valid measure for use with psychiatric outpatient populations.

The internal reliability coefficients of the nine dimensions assessed by the SCI-90 have been reported as ranging from .77 to .90 (Derogatis, Richels \& Rock, 1976). This was based on a heterogenous group of 565 psychiatric outpatients. With respect to its validity, the nine dimensions have been shown to reach peak correlations with analogous scales from among the clinical scales of the Minnesota Multiphasic Personality Irventory (MMPI) (Derogatis et al., 1976). This was based on 209 symptomatic volunteers for therapeutic drug trials. In addition, the $S C I-90$ has been shown to be a sensitive measure of a variety of treatment effects including brief therapy (Green, Gleser, Stone, \& Seifert, 1975). 
H. Beck Depression Inveritory

The Beck Depression Inventory (Beck and Steer, 1987) is a 21-item, mutiple choice questionnaire that measures the severity of depressive symptomatology. A major strength of this scale is the extensive available data concerning its psychometric properties, including normative data. Another strength is that the items reflect the affective, cognitive, and physiological components of depression. The Beck is particularly suited for use with outpatient populations given its sensitivity to mild and moderate levels of depression (Golin \& Hartz, 1979). In addition, it has been shown to be sensitive to psychotherapy treatment effects. Besides having good predictive validity, the Beck's concurrent validity is supported by its strong correlation with the Zung Self-Rating Depression Scale (Reynolds \& Gould, 1981) and clinicians' ratings of depression (Beck \& Beamesderfer, 1974). Its construct validi_ $s$ also suggested by the concordance between its items and six of the nine criteria for a depressive episode presented in the Diagnostic and Statistical Manual of the American Psychiatric Association (DSM-III). With respect to reliability data, the split-half correlation coefficient has been reported to be .86 with an internal alpha of .93 (Beck \& Bearmesderfer, 1974) •

For reasons of parsimony, the 13-iten version of the Beck was utilized in the present study. This shorter version has been shown to be highly correlated with the original scale $(\underline{r}=.93)$ and has a similarly strong internal consistency ( $\underline{r}=.83$; Reynolds \& Gould, 1981). 


\section{Global Ratings of outcome}

Four measures assessed global ratings of outcome. Iambert, Shapiro, and Bergin, (1986) advocated the inclusion of global outcome ratings, arguing that they have high face validity. The first was a global rating scale of "Life Satisfaction". The scale ranges from completely dissatisfied (1) to completely satisfied (7). The scale was completed by the patient before and after therapy and at the six-month follow-up period.

Three additional outcome measures were obtained at the posttherapy assessment. The patient and therapist were asked to provide ratings of the "Overall Usefulness of Therapy." The rating scale ranges from very little (1) to very much (7). In addition, the patient evaluated his/her satisfaction with the services received. The "Service Evaluation questionnaire" consists of seven items with a 4-point rating scale reflecting dissatistaction (1) to satisfaction (4) with the quality of care provided. The mean of the seven items provided one measure of service evaluation.

\section{Procedure}

Patients were initially assessed by a staff therapist and a supervising psychiatrist who referred patients for STG. The staff therapist explained the procedures and obtained the patient's informed consent. The patient was then assessed for psychological mindedness (PMAP) and classified as highly or marginally suitable. Next, a second 
blind independent assessor adninistered the outcome battery. The principal investigator randomly assigned pairs of patients to either immediate therapy or delayed therapy and to a particular therapist and group. Delayed therapy patients waited 12 weeks before being re-assessed with the outoome battery. Therapy lasted 12 weeks. All therapy sessions were taperecorded for subsequent process analyses. The therapist provided ratings concerning his/her perception of the therapy process after each session. Soon after therapy ended the patient met with the independent assessors who artiunistered the PMAP and outcome battery. Six months after the end of therapy the independent assessors met with the patient for a final, follow-up administration of the PMAP and outcome battery. All dropouts were requested to participate in a final interview with the independent assessors who admininstered the outcome battery and investigated the patient's reasons for leaving therapy. 


\section{Results}

The results are presented in four sections that focus on the patient sarmples available for various analyses, the analyses of pretest scores, the tests of the major hypotheses, and additional analyses. Results presented in the first and second sections primarily involve frequency data. These data were subjected to chi-square tests of independence and, where appropriate, t-tests. Results presented in the third and fourth sections involved several statistical techniques. The pretest-posttest, control group design (Campbell \& Stanley, 1963) involved a repeated measurement of outcome variables. Changes occurring over the pretest to posttest period (prescore-postscore data) were analyzed by means of multivariate and univariate statistical procedures. Several of the procedures were implemented to adjust for the common occurrence of significant linear relationships between prescores and postscores. A description of each of these statistical procedures preceeds the sections concerning findings resulting fiom their implementation.

When required, multiple comparison tests were condiuted between individual cell means using the scheffé methra which utilizes the $\underline{F}$ statistic. The level of significance crosen was .05. Marginal sjynificance was defined as an alpha level between .05 and .10. Two-tailed tests of significance were chosen for all correlation coeffirients and t-tests. The analyses were conducted using computer software packages from SAS (SAS Institute, 1985) and SPSS $^{\mathrm{X}}$ (SPSS Incorporated, 1988). 


\section{Patient Samples Available for Analyses}

A total of 79 patients participated in the study providing, at minimum, initial assessment data. There were 33 patients ranc mú assigned to the immediate treatment condition and 33 patients randomly assigried to the control condition. of the 33 patients within each condition, 19 had scored high and 14 had scored low on fsychological mindedness (PM). After the initial condition assignment, another 13 patients were added to the control condition. Five of these additional patients ( 3 highly suitable and 2 margirally suitable) replaced patients who had dropped out early in the delay period. The decision to use these patients as replacements was based on the fact that their PM score, gender and age were comparable. The other eight additional patients ( 3 highly suitable and 5 marginally suitable) were added just before the first therapy session. (On one occasion, a patient was added to the group at the second session.) They replaced patients who elected not to receive treatment after the delay period. They were accepted into the study to ensure that a sufficient number of patients were avaiiable for the control patients' therapy groups.

A number of patients dropped out at different times throughout the study. The attrition figures are presented in Table 4 . In terms of attrition according to condition assigrment, the rates are quite similar. The immediate treatment condition suffered 15 dropouts: five failed to attend one session (decliners), eight dropped out after attending less than half the sessions (early dropouts) and two terminated prematurely after attending more than half the sessions 
Table 4

Summary of Patient Attrition

\begin{tabular}{|c|c|c|c|c|c|c|c|c|c|c|c|}
\hline $\begin{array}{l}\text { Group } \\
\text { I\#|C }\end{array}$ & & $\begin{array}{c}\# \\
\text { Initially } \\
\text { Assigned }\end{array}$ & $\mid \begin{array}{c}\# \\
\text { Added } \\
\text { to Wait }\end{array}$ & $\begin{array}{c}\# \\
\text { Added } \\
\text { to Grour }\end{array}$ & $\left\{\begin{array}{c}\# \\
\text { Total } \\
\text { Assigned }\end{array}\right.$ & $\begin{array}{l}\text { \# } \\
\text { Declined } \\
\text { Therapy }\end{array}$ & $\begin{array}{c}\# \\
\text { Began } \\
\text { Therapy }\end{array}$ & $\begin{array}{l}\quad \# \\
\text { Early } \\
\text { Dropout }\end{array}$ & $\begin{array}{l}\# \\
\text { Late } \\
\text { Dropout }\end{array}$ & $\begin{array}{c}\# \\
\text { Remainers }\end{array}$ & \begin{tabular}{|l|}
\multicolumn{1}{c|}{$\#$} \\
Returned for \\
Follow-up
\end{tabular} \\
\hline 1 & 11 & & 1 & & | & & & 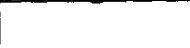 & & & \\
\hline $1 \mid I$ & H) & 6 & $V / A$ & 0 & 6 & 2 & 4 & 1 & 0 & 3 & 3 \\
\hline 1 & $\mathbf{M}$ & 3 & $\mathrm{~N} / \mathrm{A}$ & 0 & 3 & 0 & 3 & 2 & 0 & 1 & 1 \\
\hline 210 & $\mathrm{H}$ & 5 & 0 & 1 & 6 & 2 & 4 & 0 & 0 & 4 & 4 \\
\hline 1 & $\mathbf{M}$ & 4 & 0 & 2 & 6 & 4 & 2 & 1 & 1 & 0 & 0 \\
\hline $3 \mid I$ & $\mathrm{Hi}$ & 5 & $\mathrm{~N} / \mathrm{A}$ & 0 & 5 & 0 & 5 & 1 & 0 & 4 & 3 \\
\hline$i$ & $\mathbf{M}$ & 3 & $N / A$ & 0 & 3 & 1 & 2 & 0 & 2 & 0 & 0 \\
\hline $4 \mid D$ & $\mathrm{H}$ & 4 & 2 & 1 & 7 & 2 & 5 & 0 & 0 & 5 & 5 \\
\hline 1 & $\mathbf{M} \mid$ & 3 & 0 & 1 & 4 & 2 & 2 & 1 & 0 & 1 & 1 \\
\hline $5 \mid I$ & $\mathrm{H}$ & 4 & $\mathrm{~N} / \mathrm{A}$ & 0 & 4 & 0 & 4 & 0 & 0 & 4 & 4 \\
\hline 1 & $\mathbf{M}$ & 3 & $N / A$ & 0 & 3 & 0 & 3 & 3 & 0 & 0 & 0 \\
\hline 610 & $\mathrm{Hj}$ & 4 & 1 & 0 & 5 & 1 & 4 & 0 & 0 & 4 & 4 \\
\hline & $\mathbf{M} \mid$ & 3 & 2 & 0 & 5 & 2 & 3 & 0 & 1 & 2 & 1 \\
\hline $7 \mid I$ & $\mathrm{Hi}$ & 4 & N/A & 0 & 4 & 0 & 4 & 0 & 0 & 4 & 4 \\
\hline$i$ & Mi & 5 & $N / A$ & 0 & 5 & 2 & 3 & 0 & 1 & 2 & 2 \\
\hline 8iD & $\mathrm{HI}$ & 6 & 0 & 1 & 7 & 4 & 3 & 0 & 0 & 3 & 2 \\
\hline$\frac{1}{\mid \text { Total }}$ & $\frac{\mathrm{MI}}{\mathrm{HI}}$ & $\frac{4}{38}$ & $\frac{0}{3}$ & $\frac{2}{3}$ & 44 & 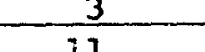 & $\frac{3}{32}$ & $\frac{1}{2}$ & $\frac{0}{0}$ & $\frac{2}{31}$ & 29 \\
\hline & MI & 28 & 2 & 5 & $\begin{array}{l}44 \\
35\end{array}$ & $\begin{array}{l}11 \\
14\end{array}$ & 21 & 8 & 5 & 8 & 7 \\
\hline
\end{tabular}

Note: C - Condition: I - Immediate treatment; D - Delayed treatment condition

PM - Psychological mindedness: H - High PM; M - Marginal PM. N/A - ivon-applicable 
(late dropouts). The control condition suffered 20 dropouts (i.e. during the delay period). The dropout rate for the immediate condition was 45.4 percent (15 of 33 patients) while the dropout rate for the control condition was 52.6 percent (20 of 38 patients). A chi-square analysis was conducted on the frequency of remainers and dropouts between the two conditions. The result of the chi-square was nonsignificant, indicating that attrition was not related to condition assigmment.

Fifty-four patients began the immediate or delayed treatment phases of the study, attending at least one of the twelve group therapy sessions. From this set of patients, there were 15 dropouts, ten early and five late. Thus, the overall dropout rate during the treatment phases was 27.8 percent. While 62 percent of the marginal PM patients dropped out, only 6 percent of the high FM patients dropped out. Table 5 summarizes the attrition rates curing the treatment phases according to the patients' level of psychological mindedness. Of the 15 dropouts, two patients were highly psychological minded while 1? were in the marginal range. The result of a chi-square analysis on these frequency counts wels highly significant, $\underline{x}^{2}(1)=17.46, p<.001$. The proportion of expliined va:iance attributable to PM was calculated (Hudson, Thyer, \& Stocks, 1985). The proportion was found to be .32 indicating a strong association between level of psychological mindedness and attrition from therapy.

The relationship between psychological mindedness and dropping out was an informative although disnutive finding. The implications of this finding will be presented in the Discussion section. It is 


\section{Table 5}

Psychological Mindedness and Attrition During Treatment Phase

\begin{tabular}{|c|c|c|}
\hline $\begin{array}{c}\text { Ier el of } \\
\text { Psychol ggica } \text { Mindedness }\end{array}$ & $\begin{array}{l}\text { Number who } \\
\text { Lemained }\end{array}$ & $\begin{array}{l}\text { Number who } \\
\text { Dropped out }\end{array}$ \\
\hline HTG 1 & 31 & 2 \\
\hline MARGINAL & 8 & 13 \\
\hline
\end{tabular}

important at this point, however, to discuss the disruptive implications that this association had for the analyses of the data. Only three of the marginally suitable patients in the immediate treatment condition actually completed treatment. Hence, the number of patients available to test the interaction effects of treatment and suitability was remarkably reduced. It was decided that the two patients who had attended the majority of their sessions before prematurely teminating (the late dropouts) would be included as "treated." One patient had dropped out after session eight and the other had dropped out after the tenth session. This decision increased the number of marginally suitable patients in the immediate treatment condition to five. The few number of treated marginally suitable patients barely permitted testing for the main effect of PM and the interaction effect of the study's two primary independent variables. A potential third independent variable was therapist, and a potential fourth, partiaily-nested, independent variable was groups. Investigations of the three-way and four-way interaction effects were, however, pre-empted given the small muber of patients per cell. While 
the three-way interaction between suitability, therapist, and group could have been investigated, the results would have been misleading. The immediately ireated and control patients could not be combined for this analysis since the wait list patients had yet to experience the group and its therapist. Investigating this three-way interaction using the data from the immediately treated patients was again prevented by the few treated marginal PM patients. Hence, the data analyses focused on the effects of the two primary independent variables, treatment and suitability.

Forty-eight patients contributed data for comparing the outcomes of the immediate treatment versus control conditions. The immediate treatment patients were included in these analyses if they had attended over half the therapy sessions, and provided pre and posttreatment data. The mean number of sessions attended by the immediately treated patients was 11 with a standard deviation of 2.7. Twenty immediately treated patients provided data for these analyses (15 high PM, 5 marginal PM). control patients were included in the data analyses if they provided prewait and postwait data. Inclusion did not require their continuation into the treatment phase of the study. Twenty-eight control patients provided data for these analyses ( 16 high PM, 12 marginal PM). Four cases had incomplete data due to the patients' failure to return for the postwait interview, or to complete their questionnaire packet.

Seventeen control patients proceeded from the wait list through the treatment phase of the study (13 high PM, 4 marginal PM), providing data for the "own control" or the "within group" comparison of 
treatment effects. (One marginal PM patient who proceeded from the wait list to the treatment phase dropped out early in therapy. Hence, his posttreatment data was inappropriate for these analyses.) In terms of follow-up data, of the 39 patients who completed treatment, 36 (92\%) returned for the follow-up assessment (29 high PM, 7 marginal PM) .

Investigations of the relationships between process and outcome were based on the data provided by 43 treated patients ( 31 high PM, 12 marginal PM). One of the treated patients (a late dropout) failed to provide posttreatment data. The data from the early sessions of the early dropouts were deleted since they were based on only a small sampling of behavior. Furthermore, any relationships that might have been found between their process and outcome data would have been confounded by the fact that they dropped out before being treated. Investigations of relationships between psychological mindedness and therapy process imvolved the data from the 43 treated patients.

\section{Pretest Score Analyses}

Statistical analyses were conducted to verify that immediately treated patients were comparable to control patients, and that high PM patients were comparable to marginal PM patients with respect to possible confounding variables. The variables included demographic characteristics, pretest oricome levels, medications used, and factors relating to their person loss(es).

The demographic variables were age, sex, marital status, level of education and enployment status. Chi-square analyses and where 
appropriate, t-tests, indicated no significant differences between the inmediate treatment and control conditions or between the high FM and marginal PM patients on these variables.

In order to rule out the possibility of significant pretest outcome differences between the treated and control patients, and the high and the marginal PM patients, two-by-two analyses of variance were conducted on the prescores of all outcome variables. No significant differences were found. This indicated that patients in the different conditions were initially comparable on the outcome variables.

In order to ascertain whether the use of medication was confounding the impact of the independent variables, chi-square analyses were conducted on the use of medications among the wait list (43\%) versus immediately treated patients (45\%) and among the highly (48\%) versus marginally suitable patients (35\%). The medications were almost entirely antidepressants. A separate two (high versus marginal PM patients) by two (wait list versus delayed treatment phase) chi-square analysis was conducted for medication use among the 17 control patients who entered treatment following the delay period. The results of the chi-squares conducted on each of these frequency counts wire nonsignificant. Hence, the use of medication was comparable for patients regardless of treatment condition or level of suitability indicating that subsequent findings of a significant treatment effect for STG would not be confounded by the differential use of medication.

Whether or not patients differed significantly on aspects associated with the loss(es) was also explored. The aspects that were investigated included the incidence of loss by death as opposed to loss 
by separation, the incidence of single versus multiple losses, the incidence of the death of a parent as opposed to another person, the length of time since the loss, and the age of the patient at which he/she experienced the Ioss. None of the (chi-square or t-test) analyses indicated a significant difference between the immediately treated versus control patients or between the high versus marginal PM patients with respect to these aspects of the loss(es).

In summary, results of the the pre-group analyses confimed that patients in each treatment condition and at both levels of suitability were comparable with respect to demographic variables and other possible confounding variables.

\section{Tests of Major Hypotheses}

Hypotineses 1,2 , and 3 concern the independent and additive effects of the treatment variable (STG) and the suitability variable (Psychological Mindedness; PM) on the dependent variables (outcome indices). Since these hypotheses were initially addressed by the same series of analyses, they will be presented in the same section.

Hypotheses 1,2 and 3.

The first hypothesis predicted a main effect for STG and the second hypothesis predicted a main effect for PM. Hypothesis 3 predicted that there would be an additive effect between treatment and suitability. The consequence of this additive effect would result in the treated high PM patients achieving the best outoome, the untreated marginal PM 
patients achieving the worst outcome, and patients in the remaining two groups (i.e. the treated marginal FM patients and the untreated high PM patients) achieving outcomes in between.

Tr test these hypotheses, an initial two factor (treatment, suitability) MANOVA was conducted on the outcome variables. The MANOVA was conducted instead of a MANCOVA since the latter technique was not feasibie. The MANOOVA program demands that the same covariate or set of covariates be used for all dependent variables. Since the covariate was each dependent variable's coinciding prescore, a procedure was needed that would allow more flexibility. Utilizing the MANOVA procedure with residual gain scores offered this flexibility while correcting for the influence of the correlation between the prescores and postscores on the raw postscores. Hence, the outcome data were converted to residual gain scores (RGS). FGS equals the Z-score of the postscore minus the product of the $\mathrm{z}$-score of the prescore times the correlation between the prescorres and postscores [i.e. RGS =

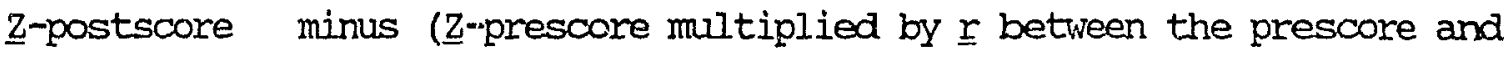
postscore) ]

The set of outcome variables available for this analysis consisted of the 17 outcome variables which were assessed both before and after therapy for the patients in the immediate treatment condition and both before and after the wait list period for the patients in the delayed treatment condition. Variables that assessed overall benefit from therapy (e.g. patient-rated Overall Usefulness of Therapy) were not appropriate for inclusion since they were only relevant for patients in the immediate treatment condition. Four of this set of outcome 
variables were further deleted from the MANOVA due to the relatively small n's for these variables. (The MANOVA procedure demands that all included patients provide complete data on all variables. A patient who has not provided complete data is automatically deleted from the analysis.) Three variables chosen for deletion were those that were nonapplicable to many patients (i.e. the Work, Partner, and Children subscales of the Modified Social Adjustment Scale). Due to occasional incomplete subscales interspersed in the data, the entire set of data for these 14 variables was available for 32 of the 48 participitating patients. In addition, the scI-90 was the most often incomplete or erroneously completed questionaire. It also was deleted from the MANOVA. This left the ratio of patients to variables at $37: 13$, which approaches the ratio of $3: 1$.

The MANOVA yielded a significant main effect for treatment, $\underline{F}(13,21)=2.8, \underline{p} \times .02$. The main effect for suitability was nonsignificant, $\underline{E}(13,21)=1.8, \underline{p} \times 11$, and the interaction effect approached significance, $\underline{F}(13,21)=1.9, \underline{p} \times 10)$. The results of the MANOVA indicated that significant findings of a treatment effect and an interaction effect from subsequent univariate analyses would not be merely a statistical artefact. To determine which outcome variables were evidencing significant effects, a two (immediate versus delayed treatment) by two (high versus manginal suitability) analysis of covariance (ANCOVA) was conducted for each of the 17 outcome variables that involved pre and postscores. The ANCOVA removes any possible confounding effects resulting from the relationship between pre and post outcome scores. In each case the dependent variable was the 
postscore and the covariate was the prescore. Each ANCOVA could provide evidence of a main effect for treatment, a main effect for suitability, and an interaction effect. Given that the MaNOVA failed to reveal a main effect for suitability, only results concerning main effects for treatment and interaction effects were interpreted from the series of ANCOVA's. Interaction effects were given priority in relation to treatment main effects. Table 6 presents a summary of these analyses. (The ANCOVA tables are reproduced in Appendix 0) .

Eight significant and one near significant main effects for treatment (in the absence of significant interaction effects) were found. The variables included: the Family of Origin, $F(1,38)=4.91$, p<.05, Partner, $\underline{F}(1,9)=3.43, \underline{p}<.10$, and Sexual, $\underline{F}(1,39)=10.11$, p<.01, subscales of the Modified Sccial Adjustment Scale (SAS-M), the Target Severity as rated by the independent assessor (TSIA), $\underline{F}(1,39)=$ 9.95, pr.01, the Avoidance subscale of the Imoact of Events Scale $($ IES-A) $, \underline{E}(I, 39)=6.74, \underline{p} \times .05$, the Autonomy subscale of the Interpersonal Dependency Inventory (IDI-Au), $\underline{\underline{F}}(1,38)=4.74, \underline{p}<.05$, the Beck Depression Inventory (Beck), $\underline{F}(1,39)=10.32, \underline{p}<.01$, the Rosenberg Self-esteem Scale (Rosenberg), $\underline{E}(1,36)=16.89, \underline{p}<.001$, and the Iife Satisfaction scale (Life Sat), $\underline{E}(1,36)=9.85, \underline{p} \times .01$. In each case, a comparison of the adjusted postscore means for the two groups indicated a more favourable outcome for the treated patients than the control patients.

A significant interaction effect for three of the variables was indicated by the results of the ANCOVA's. These variables were the Social subscale of the SAS-M, $F(1,39)=7.06, \underline{p}<.05$, the Interpersonal 
Table 6

\section{F-Ratios From Two by Two Analyses of Covariance (ANCOVA) Indicating Main and Interaction Effects of Treatment and Suitability}

\begin{tabular}{|c|c|c|c|c|}
\hline Outcome Variables & $\underline{n}$ & $\begin{array}{l}\text { Treatment } \\
\text { Effect }\end{array}$ & $\begin{array}{c}\text { PM } \\
\text { Effect }\end{array}$ & $\begin{array}{c}\text { Interactior } \\
\text { Effect }\end{array}$ \\
\hline \multicolumn{5}{|c|}{ Modified Social Adjustment Scale } \\
\hline Work & 37 & 0.23 & 0.67 & 0.12 \\
\hline Social & 44 & $3.31^{\mathrm{a}}$ & 0.14 & $7.06 *$ \\
\hline Family of Origin & 43 & $4.91 *$ & 0.14 & 0.10 \\
\hline Partner & 14 & $3.43^{\mathrm{a}}$ & 0.03 & 0.02 \\
\hline Children & 31 & 0.42 & 0.00 & 1.04 \\
\hline Sexual & 44 & $10.11 * \star$ & 2.38 & 1.01 \\
\hline \multicolumn{5}{|l|}{ Target Severity } \\
\hline Independentiy rated & 44 & $9.95 * *$ & $3.27^{\mathrm{a}}$ & 2.44 \\
\hline Patient-rated & 44 & 2.31 & 0.16 & 1.36 \\
\hline \multicolumn{5}{|l|}{ Impact of Events Scale } \\
\hline Intrusion & 44 & 1.73 & 1.38 & 0.20 \\
\hline Avoidance & 44 & $6.74 *$ & $3.57^{\mathrm{a}}$ & 0.00 \\
\hline \multicolumn{5}{|l|}{ Interperscnal Behavior Scale } \\
\hline Present Functioning & 44 & $5.13 *$ & 0.05 & $5.72 *$ \\
\hline \multicolumn{5}{|c|}{ Interpersonal Dependency Inventory } \\
\hline Emotional Reliance & 43 & 0.17 & 2.22 & 0.10 \\
\hline Autonomy & 43 & $4.74 *$ & 0.52 & 0.90 \\
\hline scL-90 Total Score & 38 & $10.40 * *$ & 0.05 & $5.02 *$ \\
\hline Beck Depression Inventory & 44 & $10.32 * *$ & 1.02 & 0.65 \\
\hline Rosenberg Self-Esteem Scale & 41 & $16.89 * * *$ & $4.89 *$ & $3.57^{\mathrm{a}}$ \\
\hline Iife Satisfaction Scale & 41 & $9.85 * *$ & $3.45^{\mathrm{a}}$ & 1.21 \\
\hline
\end{tabular}

Note: PM - Psychological Mindedness

$$
\begin{aligned}
a-p & <.10 \\
\star-p & <.05 \\
\star *-p & <.01 \\
* * *-p & <.001
\end{aligned}
$$


Behavior Scale - Present Functioning (IBSP), $\underline{F}(1,39)=5.72, \underline{p}<.05$, and the SCI-90 Total score $(S C L-90), \underline{F}(I, 33)=5.02, \underline{p}<.05$. Multiple comparison tests (Scheffé) among the four cells of the design indicated that for all three variables, the marginal PM patients in the immediately treated condition had a more favourable outcome than marginal PM patients in the control condition: Social, $F(3,39)=2.54$, $\underline{p}<.10 ; \operatorname{IBSP}, \underline{E}(3,39)=2.63, \underline{p}<.10 ; \operatorname{SCI}-90, \underline{F}(3,33)=3.47, \underline{p}<.05 .$. No other significant differences existed.

Hypothesis 1 - surpplementary Analyses. The series of ANCOVA's indicated that the immediately treated patients were reporting more improvement on outcome measures compared to patients in the control condition. In order to determine whether the improvements evidenced by the inmediately treated patients actually represented significant treatment benefits (i.e. significant improvement over the pretreatment to posttreatment period) a series of correlated t-tests were next conducted. Table 7 presents the mean and standard deviation for each of the relevant outcome variable's prescores and postscores. The $t$-value associated with the change from the prescores to postscores is also presented. For those variables that evidenced more improvement compared to the control condition (main effect for treatment revealed in the ANCOVA's), the following also evidenced a significant treatment effect (i.e. significant pre to posttreatment change): the three subscales (Family of Origin, Partner, and Sexual) of the SAS-M, TSIA, IFS-A, the Beck, and Iife Sat. Hence, the improvements on outcome variables for the inmediately treated patients represented a significant treatment effect which was also greater than the 
Table 7

Mean and $t$-Value of

Prescore to Postscore Change on Relevant outcome Variables for Patients in the Immediate Treatmant Condition

\begin{tabular}{|c|c|c|c|c|c|c|}
\hline \multirow{2}{*}{ outcome Variables } & \multicolumn{2}{|c|}{ Prescore } & \multicolumn{2}{|c|}{ Postscore } & \multirow[b]{2}{*}{$\underline{n}$} & \multirow[b]{2}{*}{$t$} \\
\hline & $\underline{\mathbf{M}}$ & $\underline{\mathrm{SD}}$ & $\underline{M}$ & $\underline{S D}$ & & \\
\hline \multicolumn{7}{|l|}{ SAS-M } \\
\hline Family of origin & 4.5 & 1.7 & 3.6 & 1.3 & 19 & $-3.08 * *$ \\
\hline Partner & 4.9 & 1.7 & 3.7 & 1.4 & 07 & $-4.53 * *$ \\
\hline Sexual & 6.7 & 4.3 & 4.4 & 3.6 & 19 & $-2.46 *$ \\
\hline TSIA & 4.0 & 0.8 & 2.5 & 1.2 & 20 & $-6.15 * * *$ \\
\hline IES-A & 16.5 & 8.2 & 9.0 & 7.5 & 20 & $-3.40 * *$ \\
\hline $\mathrm{IDI}-\mathrm{Au} \mathrm{u}^{\wedge}$ & 2.0 & 0.4 & 2.0 & 0.4 & 20 & 1.07 \\
\hline Beck & 11.2 & 8.0 & 5.4 & 4.4 & 20 & $-4.20 * * *$ \\
\hline Rosenberg & 3.4 & 1.4 & 2.4 & 1.9 & 19 & $-1.91^{\mathrm{a}}$ \\
\hline Life Sat^ & 3.6 & 1.7 & 4.7 & 1.3 & 20 & $3.29 * *$ \\
\hline
\end{tabular}

Note: ^ Lower scores reflect more disturbance on these measures

$$
\begin{aligned}
a-p & <.10 \\
*-p & <.05 \\
* *-p & <.01 \\
* * *-p & <.001
\end{aligned}
$$

SAS-MEModified Social Adjustment Scale; TSIA=Independently-Rated Target Severity; IES-A=Avoidance subscale of Impact of Event Scale, IDI-Au = Autonomy subscale of Interpersonal Dependency Inventory, Beck=Beck Depression Inventory, Rosenberg=Rosenberg Self-esteem Scale, Life Sat $=$ Life Satisfaction Scale. 
improvements reported by patients in the wait list control condition.

It was possible, however, that the control group had also experienced significant improvements over the wait period but that their improvements were smaller than those of the inmediately treated patients. To explore this possibility, a second series of correlated t-tests were conducted on the same variables for the control group patients. Table 8 presents the mean and standard deviation for each outcome variable's prescores and postscores, and the t-value associated with the change over the wait period. The results indicated that for the control patients, only the Beck and ISIA showed significant improvement while the Rosenberg showed significant worsening. Hence, the control patients evidenced few significant improvements over the wait period.

Magnitude of Effect. It has been asserted that statistical significance confounds the magnitude of the effect produced by the treatment with the size of the sample, and other technical features of the experiment that are independent of the treatment fifect (Smith, Glass \& Miller, 1980). To control for these confounding influences, Smith et al. (1980) proposed the calculation of a treatment effect size. They defined the effect size (ES) as the mean difference between the treated and control subjects' scores divided by the standard deviation of the control group. The ES represents, therefore, the difference in standard deviation units between the means of the treated and control groups. By converting the standand deviation units into area percentage of the normal curve, the ES permits a comparison between the average treated patient and the control group on each 
Table 8

Mean and $t$-Value of

Prescore to Postscore change on Relevant Outcome Variables for Patients in the control condition

\begin{tabular}{|c|c|c|c|c|c|c|}
\hline & Pr & core & POS & ore & & \\
\hline outcome Variables & $\underline{M}$ & $\underline{S D}$ & $\underline{M}$ & $\underline{\mathrm{SD}}$ & $\underline{n}$ & $t$ \\
\hline$A S-M$ & & & & & & \\
\hline $\begin{array}{l}\text { Family of Origin } \\
\text { Partner } \\
\text { Sexual }\end{array}$ & $\begin{array}{l}4.4 \\
5.3 \\
6.2\end{array}$ & $\begin{array}{l}1.2 \\
2.1 \\
4.5\end{array}$ & $\begin{array}{l}4.3 \\
5.5 \\
6.9\end{array}$ & $\begin{array}{l}1.6 \\
2.7 \\
4.1\end{array}$ & $\begin{array}{l}24 \\
07 \\
25\end{array}$ & $\begin{array}{r}-0.22 \\
0.41 \\
1.23\end{array}$ \\
\hline TSIA & 4.2 & 0.5 & 3.7 & 1.2 & 24 & $-2.74 *$ \\
\hline IES-A & 13.2 & 7.3 & 13.2 & 9.4 & 24 & -0.01 \\
\hline IDI-Au & 1.8 & 0.4 & 1.8 & 0.4 & 23 & -0.94 \\
\hline Beck & 12.1 & 7.6 & 9.7 & 6.5 & 24 & $-2.91 * *$ \\
\hline Rosenberg & 3.0 & 2.1 & 3.7 & 2.0 & 22 & $4.95 * * *$ \\
\hline Iife sat & 3.0 & 1.5 & 3.4 & 1.4 & 21 & 1.09 \\
\hline
\end{tabular}

Note: $a-p<.10$

$*-\underline{p}<.05$

$* *-\underline{p}<.01$

$* * *-\underline{p}<.001$

SAS-MFNodified Social Adjustment Scale; TSIA=Independently-Rated Target Severity; IES-A=Avoidance subscale of Impact of Event Scale, IDI-Au = Autonomy subscale of Interpersonal Dependency Inventory, Beck=Beck Depression Inventory, Rosenberg=Rosenberg Self-esteem Scale, Life Sat=Iife Satisfaction Scale. 
outcome variable. For example, an ES of 1.0 represents a difference of one standard deviation unit between the the mean of the treatment group and the mean of the control group or that the average treated patient is better off than 84 percent of the control group (a standard score of I in the normal curve provides the upper bound to $84 \%$ of the area). Utilizing the adjusted postscore means, the treatment effect size was calculated for each outcome variable in the present study (ES = adjusted postscore mean of the treated group minus the adjusted postscore mean of the control group, divided by the square root of the adjusted mean square error). In addition, the aggregated effect size for STG was computed by averaging tine effect size for all outcome variables. Finally, the percentage of area of the nomal curve that corresponded with the effect size was also determined. As presented in Table 9, the effect size was above .70 for 11 of the 17 outcome variables. These variables consistod of the Family, Partner, and sexual subscales of the SAS-M, the TSIA, the IES-A, the IBSP, the IDI-Au, the SCI-90, the Beck, the Rosenberg, and the life Sat. The largest ES, 1.50, was for the Rosenberg, indicating that the average treated patient was better off than 93 percent of the control patients in terms of self-esteem. The smallest ES, .14, was for the IDI-ER, indicating that the average treated patient was better off than 56 percent of the control patients in terms of being emotionally reliant. The aggregated effect size was .79, indicating that, overall, the average treated patient was better off than 78 percent of the control patients.

Clinical Significance. The pre to post improvements evidenced by 


\section{Table 9}

Specific and Agrregated Effect Size

$\frac{\text { and corresponding Area Percentage of Normal curve }}{\text { for Treated Versus Control Patients }}$

\begin{tabular}{|c|c|c|}
\hline outcome variabies & Effect Size & of of Normal curve \\
\hline $\begin{array}{l}\text { SAS-M } \\
\text { Work } \\
\text { Social } \\
\text { Family of Origin } \\
\text { Partner } \\
\text { Children } \\
\text { Sexual }\end{array}$ & $\begin{array}{r}.17 \\
.61 \\
.75 \\
1.01 \\
.24 \\
1.06\end{array}$ & $\begin{array}{l}56.8 \\
72.9 \\
77.3 \\
84.4 \\
59.5 \\
85.5\end{array}$ \\
\hline $\begin{array}{l}\text { TSIA } \\
\text { TSPI }\end{array}$ & $\begin{array}{r}1.10 \\
.51\end{array}$ & $\begin{array}{l}86.4 \\
69.5\end{array}$ \\
\hline $\begin{array}{l}\text { IES-I } \\
\text { IES-A }\end{array}$ & $\begin{array}{l}.45 \\
.88\end{array}$ & $\begin{array}{l}67.4 \\
81.1\end{array}$ \\
\hline IBSP & .78 & 78.2 \\
\hline $\begin{array}{l}\text { IDI-ER } \\
\text { IDI-AlI }\end{array}$ &. .74 & $\begin{array}{l}55.6 \\
77.3\end{array}$ \\
\hline SCI-90 & 1.21 & 88.7 \\
\hline Beck & 1.12 & 86.9 \\
\hline Rosenberg & 1.50 & 93.3 \\
\hline Life Sat & 1.16 & 87.7 \\
\hline Aggregated Effect Siz & .79 & $\overline{78.5}$ \\
\hline
\end{tabular}

SAS-M $=$ Modified Social Adjustment Scale; TSIA=IndependentIy-Rated Target Severity; TSPT=Patient-Rated Target Severity, IES-I=Intrusion subscale of Impact of Event Scale, IFS-A=Avoidance subscale of Imqact of Event Scale, IBSP=Present Functioning subscore of Interpersonal Behavior Scale, IDI-ER=Emotional Reliance subscale of Interpersonal Dependency Inventory, IDI-Au=Autonony subscale of Interpersonal Dependency Inventory, SCI-90=Total Score of $S C I-90$, Beck=Beck Depression Inventory, Rosenberg=Rosenberg Self-esteem Scale, Iife Sat=Life Satisfaction Scale. 
the inmediately treated patients were next considered from the point of view of clinical significance. Clinical significance refers to the meaningfulness of the treatment effect in terms of substantially improving the patient's quality of life, or rendering him/her within the normal range of functioning. In terms of the scales' anchor points, scores on the Family and Partner subscales of the SAS-M had decreased from the "somewhat" disturbed range to the "slightly" disturbed range. Sexual (SAS-M) disturbance had diminished from "moderate" to "somewhat". Similarly, ISIA had improved from the "considerable" severity range to between the "minor" and "moderate" severity range. With respect to IES-A, the immediately treated patıents were reporting that their conscious avoidance of themes associated with the loss(es) had also decreased. The average item frequency ratings had improved from between the "rarely" and "sometimes" range to between the "not-at-all" and "rarely" range. According to the Beck scores, patients' level of depression had been reduced from "moderate" to "mild." Globally, on the Life sat patients were endorsing digits on the "more satisfied" end of the scale than the pretreatment endorsements of digits on the "more dissatisfied" end of the scale. The change in the average anchor point endorsements suggested that the immediately treated patients were clinically less disturbed in their functioning. It was still debatable, however, whether these changes signified an improved quality of life.

Jacobson and Revenstorf (1988) have developed three methods to quantitatively determine the clinical significance of treatment effects. The first method involves calculating a cut-off point at 
which a patient is equally likely to be a member of either the functional or dysfunctional distributions. It is a point between the means of these two populations. The formula for calculating the cut-off point has in the mumerator the standard deviation of the normative population multiplied by the mean of the dysfunctional population (prescore) which is added to the product of the standard deviation of the dysfunctional population (prescore) times the mean of the normative population. In the denominator is the sum of the two standard deviations. The formula is, therefore, $\left(S_{0} \underline{M}_{t}\right)+\left(S_{t}\right.$ $\left.\underline{M}_{0}\right) / S_{0}+s_{t}$. A postscore that surpasses this cut-off point meets the cut-off criterion of clinical significance.

As a second method a t-test can be conducted between the postscores of the immediately treated patients and the scores of the normative group. In this case a nonsignificant t-test indicates clinical significance.

When norms are not availabie, a third method for determining clinical significance can be utilized. The third criterion demands that the treated patients' postscores be two standard deviations (in the direction of functionality) beyond the mean of the treated patients' prescores. In other words, the mean of the prescores minus the mean of the postscores divided by the standard deviation of the prescores must equal or be greater than two standard deviation units in order to meet this stringent criterion of clinically significant change.

Clinical significance was calculated for each cutcome variable for the immediately treated patients. When norms were available, the 
appropriate cut-off point was determined and then compared with the mean postscore. In addition to the cut-off criterion, the t-test criterion was also utilized. Normative data were available for the IES (2ilberg, Weiss, \& Horowitz, 1982), the IDI (Hirschfeld et al., 1977), the SCI-90 (Derogatis et al., 1977), the Beck (Beck \& Steer, 1987), and the Rosenberg (Kernaleguen \& Conrad, 1980). When nomative data were unavailable, the two standand deviation criterion was implemented.

For those measures for which norms were available, the results indicated (Table 10) that changes evidenced on the IFS-A and the IDI-Au reflected clinically significant improvement. The t-tests conducted on these two measures supported the finding that by the end of treatment, the treated patients were not significantly different from the normative group. In adaition, a t-test conducted for the IES-I indicated that while the mean postscore did not surpass the cut-off point, the irmediately treated group was not significantly different from the normative group by the end of treatment. On the IDI-ER, the SCI-90, the Beck and the Rosenberg, the treated patients remained significantly more disturbed than the normative group.

It was possible that the lack of clinically significant changes on these latter measures was due to the prescores being already within the normative range. However, for the IDI-ER, the mean prescore for this sample (47.16) was comparative to the mean score of two samples of mixed psychiatric patients $(48.7$ and 43.3$)$ reported by Hirschfeld et al. (1977). With respect to the SCI-90, scores of this study's sample (1.44) exceeded scores reported by Derogatis et al. (1977) for psychiatric outpatients (1.26). On the Beck, the mean prescore (18.17) 
Table 10

Clinical Significance of Treatment Effects

Utilizing Normative Comparisons

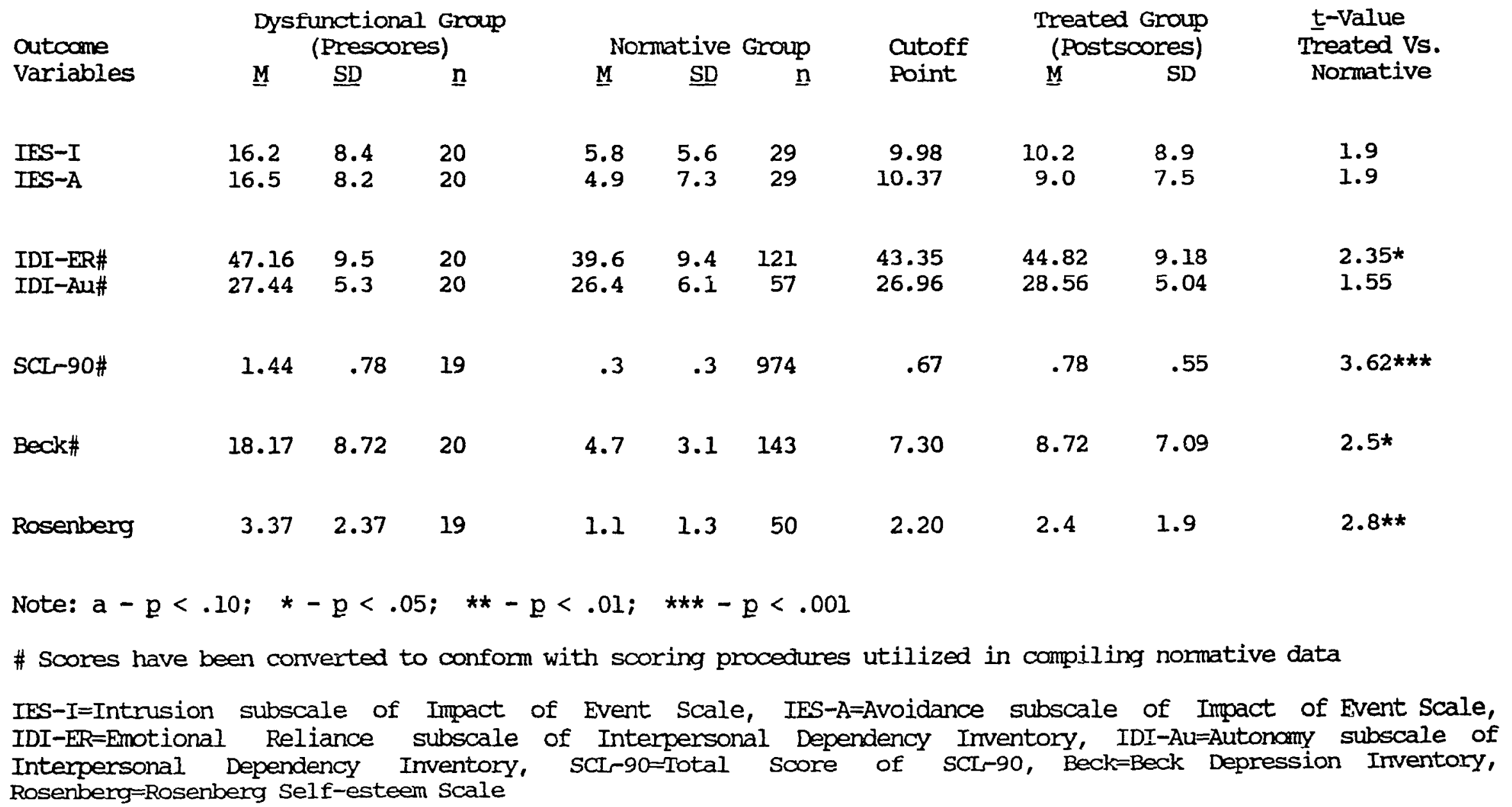


was consistent with that of patients who had received a diagnosis of dysthymic disorder (17.5) (Beck \& Steer, 1987). Finally, on the Rosenberg, this study's sample (3.37) had more problems of self-esteem than bereaved women who had sought psychotherapy (1.14) (T,jeberman \& Videka-Sherman, 1986). It should be noted the patients in this study were also comparable to two samples of psychiatric patients of mixed diagnoses on the IDI-Au (27.4 versus 27.0 and 29.2 ; Hirschfeld et al., 1977). On the Impact of Event Scale, their scores exceeded those of adult offspring of deceased parents two months after the death (16.2 versus 13.1 for Intrusion, and 16.5 versus 8.3 for Avoidance) (Zilberg, Weiss, \& Horowitz, 1982). In sumary, the patients in this sample represented a clinical population on these measures. The failure of the treatment to render the immediately treated patients within the nomative range was not a statistical artefact.

For those measures for which normative data were not available, the results indicated (Table 11) that only improvements on the TSIA and TSPT exceeded one standard deviation unit, approacining clinical significance. The changes evidenced on all subscales of the SAS-M, the IBSP and the Iife Sat did not reflect significant clinical improvement utilizing the two standard deviation criterion of change.

To summarize, the results of the statistical analyses strongly supported hypothesis 1. A significant main effect for treatment was revealed by the MANOVA. The series of univariate analyses (ANCOVA) revealed that eight of the set of 17 outcome variables evidenced a significant main effect for treatment, and a ninth variable approachea significance. Hence, the immediately treated patients were evidencing 
Table 11

Clinical Significance of Treatment Effects utilizing the Two Standard Deviation Criterion

Since Nonms were Unavailable

\begin{tabular}{|c|c|c|c|c|c|}
\hline \multirow{2}{*}{ outcome Variables } & \multicolumn{3}{|c|}{ Prescore } & \multirow{2}{*}{$\begin{array}{c}\text { Postscore } \\
M\end{array}$} & \multirow[b]{2}{*}{ SD Units } \\
\hline & $\underline{\underline{n}}$ & $\underline{M}$ & $\underline{S D}$ & & \\
\hline \multicolumn{6}{|l|}{ SAS-M } \\
\hline Work & 16 & 4.8 & 1.9 & 3.7 & .56 \\
\hline Social & 19 & 4.9 & 2.2 & 4.2 & .33 \\
\hline Family of origin & 19 & 4.5 & 1.7 & 3.6 & .53 \\
\hline Partner & 07 & 4.9 & 1.7 & 3.7 & .67 \\
\hline Children & 13 & 3.5 & 1.4 & 3.2 & .22 \\
\hline Sexual & 19 & 6.7 & 4.3 & 4.4 & .54 \\
\hline TSIA & 20 & 3.9 & 0.8 & 2.5 & $1.9^{a}$ \\
\hline TSPT & 20 & 4.0 & 0.7 & 2.9 & $1.4^{\mathrm{a}}$ \\
\hline IBSP & 20 & 126.4 & 18.2 & 130.6 & -.23 \\
\hline Iife Sat & 20 & 3.0 & 1.7 & 4.7 & -.69 \\
\hline
\end{tabular}

Note: a approached clinical significance

SAS-M=Modified Social Adjustment Scale; TSIA=Independently-Rated Target Severity; TSPI=Patient-Rated Tanget Severity, IBSP=Present Functioning subscore of Interpersonal Behavior scale, Life Sat=Life Satisfaction Scale. 
greater pre to post improvements than were the control patients. In addition to evidencing better outcomes relative to the control patients, a series of correlated t-tests revealed that the immediately treated patients were evidencing significant pre to posttherapy changes on seven of these nine targeted variables with an eighth variable approaching significance. The improvements of the inmediately treated patients were not parallelled by the control patients. Rather, the control patients evidenced significant pre to postwait improvement on only one variable, the Beck, while evidencing significant pre to post deterioration on the Rosenberg. The overall effect size of STG was .79. While statistical analyses of the pre to posttreatment improvements of the immediately treated patients confimed their significance, the clinical significance of these changes was substantiated quantitatively for only the IFS-I, IES-A, IDI-Au, TSIA and TSPT.

Delayed Treatment Phase Versus Wait List Phase. The outcome of the delayed patients after the treatment phase relative to improvements after the wait period was also investigated. This imvestigation involved conducting a series of correlated t-tests on two sets of resicual gain scores for the 17 outcome variables that were assessed before and after the delay period and after treatment. The first set of residual gain scores represented the changes that had occurred over the wait period (i.e. prewait to postwait). The second set represented the changes that had occurred over the treatment phase (i.e. postwait/pretreatment to posttreatment). The data for these analyses were provided by the 17 patients who had proceeded from the wait list to the end of the treatment phase. 
Table 12

Mean and $t$-Value from

correlated t-tests on outcome

at Three Temporal stages

for Delayed Treatment Patients

\begin{tabular}{|c|c|c|c|c|c|c|c|}
\hline outcome & $\underline{n}$ & $\underset{M}{\text { Time }}$ & $1 \underset{M}{\text { Timez }}$ & $\underset{M}{\text { Time3 }}$ & $t$-wait $t$ & $\begin{array}{c}\text { Change } \\
\text { C-treatment }\end{array}$ & t-residual \\
\hline \multicolumn{8}{|l|}{ SAS-M } \\
\hline Work & 13 & 4.6 & 4.5 & 3.4 & .16 & 1.70 & 1.74 \\
\hline Social & 17 & 6.5 & 5.1 & 5.2 & $3.10 * *$ & -.24 & $-1.76^{\mathrm{a}}$ \\
\hline Family & 16 & 4.7 & 4.6 & 3.9 & .63 & 1.61 & 1.19 \\
\hline Partner & 03 & 6.4 & 7.0 & 3.6 & $-3.21^{\mathrm{a}}$ & 2.88 & $3.44^{\mathrm{a}}$ \\
\hline Children & 12 & 4.2 & 4.7 & 3.8 & -1.05 & 2.22 & $2.81 *$ \\
\hline Sexual & 17 & 7.2 & 7.6 & 6.7 & -.55 & .87 & .94 \\
\hline TSIA & 16 & 4.2 & 3.7 & 2.7 & $1.90^{\mathrm{a}}$ & $3.11 * *$ & 1.54 \\
\hline TSPT & 17 & 4.0 & 3.4 & 2.8 & $2.06^{\mathrm{a}}$ & $2.99 * *$ & .94 \\
\hline IES-I & 17 & 17.6 & 15.3 & 14.9 & 1.37 & -.18 & -.41 \\
\hline IES-A & 17 & 13.3 & 12.8 & 12.4 & .42 & .16 & -.01 \\
\hline IBSPn & 17 & 119.1 & 115.6 & 120.6 & 1.03 & $-1.85^{a}$ & -1.61 \\
\hline IDI-ER & 17 & 2.5 & 2.5 & 2.5 & -0.08 & .45 & .37 \\
\hline IDI-Au^ & 17 & 1.9 & 1.8 & 1.7 & 1.34 & 1.12 & .45 \\
\hline$S C L-90$ & 14 & 125.9 & 113.2 & 94.9 & .75 & 1.44 & .48 \\
\hline Beck & 17 & 13.6 & 10.6 & 8.2 & $2.92 *$ & $2.12 *$ & .11 \\
\hline Rosenberg & 17 & 3.5 & 4.1 & 2.5 & $-4.40 * \star \star *$ & $3.86 * *$ & $4.71 * \star *$ \\
\hline Iife Sat^ & 16 & 2.6 & 3.0 & 4.4 & -.98 & $-4 \cdot 74 * \star \star *$ & $-2.96 * *$ \\
\hline
\end{tabular}

Note: ^ Lower scores reflect more disturbance

$\mathrm{a}-\mathrm{p}<.10, *-\mathrm{p}<.05, * *-\mathrm{p}<.01, * * *-\mathrm{p}<.001$

Timel - prewait, Time2 - postwait/pretreatment, Time3 - posttreatment

SAS-M=Modified Social Adjustment Scale; TSIA=Independently-Rated Target Severity; TSPI=Patient-Rated Target Severity, IES-I-Intrusion subscale of Impact of Event Scale, IES-A=Avoidance subscale of Impact of Event Scale, IBSP=Present Functioning subscore of Interpersonal Behavior Scale, IDI-ER=Emotional Reliance subscale of Interpersonal Dependency Inventory, IDI-Au=Autonomy subscale of Interpersonal Dependency Inventory, SCI-90=Total Score of $S C I-90$, Beck=Beck Depression Inventory, Rosenberg=Rosenberg Self-esteem Scale, Life Sat=Iife Satisfaciion Scale. 
Table 12 presents the mean for each outcome score at each of the three aforementioned assessment periods. Three types of $t$-values are also presented: the t-value associated with changes evidenced over the delay period ( $t$-wait), over the treatment period ( $t$-treatment) and over the treatment phase relative to over the wait period (t-residual). The results indicated three significant and one near significant treatment effects. The variables consisted of the Partner, $t(1)=3.44, p<.10$, and Children, $\underline{t}(10)=2.81$, pr.05, subscales of the SAS-M, the Rosenberg, $\underline{t}(15)=4.71, \underline{p}<.001$, and Life Sat, $t(14)=-2.96, \underline{p}<.01$. For 12 of the variables, the change was in the predicted direction. The Social subscale of the SAS-M evidenced a deterioration after treatment, $\underline{t}(15)=-1.76, \mathrm{p} \times .10$. It should be noted, however, that this was one of the variables which evidenced significant improvement over the wait period, $\underline{t}(15)=3.10, \mathrm{p}<.01$.

In sumary, the results of the "own control" comparison of outcome, indicated that patients who proceeded from the wait list through the treatment phase evidenced contimual irmorovement on 16 of the 17 outcome variables. Three variables evidenced significant benefits after treatment relative to changes cocurring over the wait period. These results represented some support for a treatment effect.

Hypothesis 2 - Supplementary Analyses. The findings did not support hypothesis 2 . While the suitability variable, psychological mindedness significantly predicted attrition from therapy, it was not related to outcome. The results of the MANOVA failed to find a main effect for PM.

To investigate the strength of the association between the other 
patient predictor variable, Interpretation comprehension (IC), and outcome, partial correlation coefficients were calculated for the two sets of variables. This statistical procecure controls for the effect of the relationship between the outcome variables' pre and postscores by partialling out the effect of the prescore for both the IC ratings and the outcome postscores before currelating the two sets of variables. Data for this analysis were provided by the 43 treated patients. The calculations involved the three IC variables from the first scenario of the PMAP and the entire set of outcome variables. As described in the Method section, the IC variables assessed the patients' ability to understand the actor-therapist on the PMAP videotape when he interpretated the actress-patient's problem. They consisted of Number of Dynamics, Speed of Dynamics, and Speed of Transference. The outcome variables consisted of the previously described set of 17 outcome variables that were assessed pre and posttreatment plus four variables that were only administered posttreatment. These additional four variables assessed the patient's overall benefit from treatment. They were appropriate for inclusion since this analysis addressed data from all treated patients. These latter variables included the overall Usefulness of Therapy as rated both by the patient (OUP), and by the therapist (OUT), the patient-rated Change in Target Severity (TSPC), and the patient-rated Service Evaluation Questionnaire (Serv Eval). Pearson, rather than partial, correlation coefficients were calculated for these latter four variables.

The correlation coefficients (Table 13) revealed a weak pattern of 
Table 13

Correlation coefficients for Interpretation comprehension and outcome variables

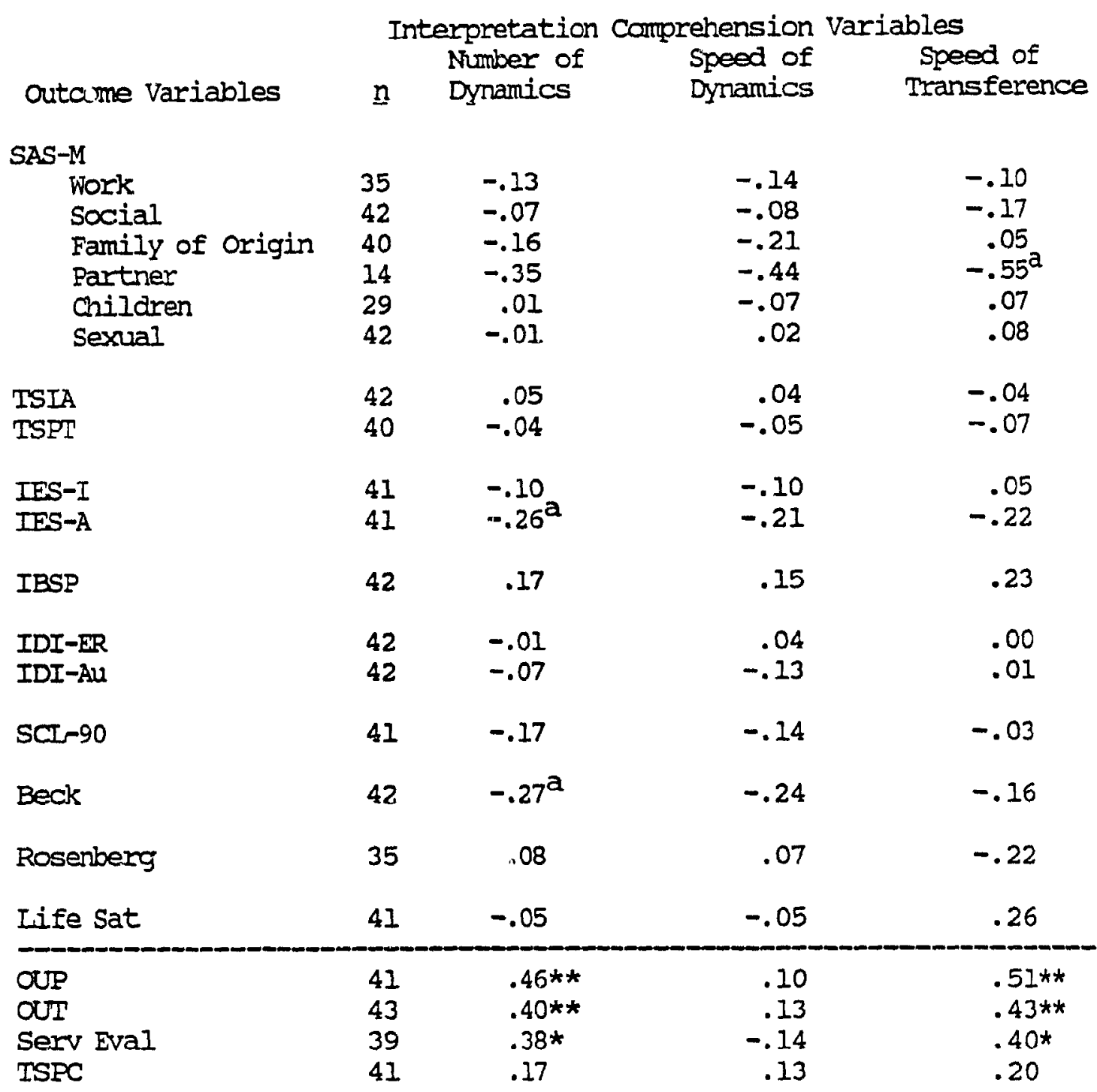

Note: $\mathrm{a}-\mathrm{p}<.10, *-\mathrm{p}<.05, * *-\mathrm{p}<.01, * * *-\mathrm{p}<.001$

SAS-M=Modified Social Adjustment Scale; TSIA=Indeperdifitly-Rated Target Severity; TSPI=Patient-Rated Target Severity, IES-I=Intrusion subscale of Impact of Event Scale, IES-A=Avoidance subscale of Impact of Event Scale, IBSF=Present Functioning subscore of Interpersonal Behavior Scale, IDI-ER=Emotional Reliance subscale of Interpersonal Dependency Inventory, IDI-Au=Autonomy subscale of Interpersonal Dependency Inventory, SCI-90=Total Score of SCI-90, Beck=Beck Depression Inventory, Rosenberg-Rosenberg self-esteem Scale, Iife Sat=Life Satisfaction Scale, oup-Patient-rated overall Usefulness of Therapy, OUT-Therapist-rated overall Usefulness of Therapy, Ser Eval-Service Evallation, TSPC-Patient-ra'ed Change in Target Severity. 
associations between the Interpretation comprehension variables and the pre-post outcome variables with none of the variables reaching statistical significance. There were, however, siginificant relationships between If and the global outcome variables. The results indicated that the more interpreted dynamics that the patient understood (Number of Dynamics), the more the patient $[\underline{r}(39)=.45$, $\underline{p}<.01]$ and the therapist $[\underline{r}(41)=.40, \underline{p}<.01]$ rated the therapy as being useful for the patient and the more satisfied the patient was with the services he/she had received $[\underline{r}(37)=.38, \underline{p}<.05]$. Similarly, the ease with which the patient had understood the actor-therapist's interpretation of transference phenomena (Speed of Transference) was directly related to those same globa] appraisals of outcome [OUP: $\underline{r}$ (39) $=.51, \underline{p}<.01 ;$ OUT: $\underline{r}(41)=.43, \mathrm{p}<.01$; Serv Eval: $\underline{r}(37)=.40 . \underline{p}<.05]$. In summary, whi'e the relationship between the IC and pre-post outcome variables was generally weak, they were associated with positive, global impressions of the therapy experience.

Hypothesis 3 - Supplementary Analyses. Hypothesis I had predicted an additive effect between the treatment and suitability variables. The analyses reported thus far partially addressed the third hypothesis. The series of ANCOVA's revealed that three variables had evidenced a significant interaction effect (Social subscale of the SAS-M, IBSP, and SCI-90). The rank order of the outcomes for these variables placed the immediately treated marginal PM patients as the best and the wait list marginal PM patients as the worst with the other two groups (the immediately treated high PM patients and the wait Iist high PM patients) in between. The only significant difference 
occurred, therefore, between the outcomes of the immediately treated versus the wait list marginal PM patients. Hypothesis 3 had onrrectly predicted that the marginal PM in the control condition would evidence the worst outcomes relative to the other three cells. This was clearly the case. Incieed, these results indicated that the marginal PM patients deteriorated somewhat over a wait list control period. Contrary to the prediction, however, these findings indicated that when the marginal FM patients were treated irmediately, they were able to benefit from the experience.

To investigate the rank ondering of each cell on the remaining 14 pre-post outcome variables, a series of scheffé planned comparison tests were conducted on the means of the adjusted postscores for the immediately treated high PM patients and the marginal PM patients in the delay condition. Table 14 presents the cell means ror the adjusted postscores of each outcome variable. Table 15 sunmarizes the rank ordering of each of the four cells on each of the 17 outcome variables. For four variables, two of the cells evidenced equal means. These variables consisted of the Family and Partner subscales of the SAS-M, and both scales of the IDI. When this equality interfered with detemining first or last rank order, the variables were deleted from consideration.

Tables 14 and 15 indicate that the best outcomes were evidenced by the immediately treated, high PM patients on six of the outcome variables (Partner, Children, and Sexual subscales of the SAS-M, the IES-I and the IES-A, and the Beck). Results of the series of Scheffe planned comparison tests indicated that for two of these variables, the 
Table 14

Cell Means of Adjusted Postscores of all outcome Variables

\begin{tabular}{|c|c|c|c|c|c|c|c|c|}
\hline \multirow[b]{4}{*}{ outcome Variables } & \multicolumn{8}{|c|}{ Treatment and suitability condition } \\
\hline & Im & dia & Treat & ent & \multicolumn{4}{|c|}{ Delayed Treatment } \\
\hline & \multicolumn{2}{|c|}{ High PM } & \multicolumn{2}{|c|}{ Marginal PM } & \multirow{2}{*}{\multicolumn{2}{|c|}{ High $\mathrm{PM}$}} & \multicolumn{2}{|c|}{ Marginal PM } \\
\hline & $M$ & $n$ & $\mathrm{M}$ & $n$ & & & $\mathrm{M}$ & $n$ \\
\hline SAS-M & & & & & & & & \\
\hline Work & 3.6 & 11 & 3.3 & 5 & 4.1 & 15 & 3.4 & 6 \\
\hline Social & 4.6 & 14 & 3.1 & 5 & 4.2 & 16 & 5.4 & 9 \\
\hline Family of Origin & 3.7 & 14 & 3.4 & 5 & 4.4 & 15 & 4.4 & 9 \\
\hline Fartner & 3.8 & 04 & 4.0 & 3 & 5.3 & 04 & 5.3 & 3 \\
\hline Children & 3.1 & 08 & 3.6 & 5 & 4.0 & 11 & 3.4 & 7 \\
\hline Sexual & 3.8 & 14 & 4.4 & 5 & 5.9 & 16 & 8.3 & 9 \\
\hline ISIA & 2.8 & 15 & 1.6 & 5 & 3.4 & 26 & 3.3 & 8 \\
\hline TSPI & 3.0 & 15 & 2.4 & 5 & 3.1 & 16 & 3.4 & 8 \\
\hline IES-I & 9.6 & 15 & 13.6 & 5 & 14.0 & 16 & 15.8 & 8 \\
\hline IES-A & 6.2 & 15 & 10.8 & 5 & 12.4 & 16 & 16.7 & 8 \\
\hline IBSP & 127.7 & 15 & 139.3 & 5 & 128.4 & 16 & 114.3 & 8 \\
\hline IDI-ER & 2.4 & 15 & 2.6 & 5 & 2.4 & 16 & 2.6 & 7 \\
\hline$I D I-A u$ & 2.0 & 15 & 2.1 & 5 & 1.8 & 16 & 1.8 & 7 \\
\hline SCI-90 & 77.0 & 15 & 34.4 & 4 & 91.0 & 13 & 127.2 & 6 \\
\hline Beck & 5.1 & 15 & 5.3 & 5 & 8.2 & 16 & 10.6 & 8 \\
\hline Rosenberg & 2.7 & 15 & 0.5 & 4 & 3.9 & 15 & 3.3 & 7 \\
\hline Life Sat & 4.3 & 15 & 5.6 & 5 & 3.4 & 15 & 3.7 & 6 \\
\hline
\end{tabular}

SAS-M=Modified Social Adjustment Scale; TSIA=Independently-Rated Target Severity; TSPI=Patient-Rated Target Severity, IES-I=Intrusion subscale of Impact of Event Scale, IES-A=Avoidance subscale of Impact of Event Scale, IBSP=Present Functioning subscore of Interpersonal Behavior Scale, IDI-ER=Emotional Reliance subscale of Interpersonal Dependency Inventory, IDI-Au=Autonamy subscale of Interpersonal Dependency Inventory, SCI-90=Total Score of SCI-90, Reck=Beck Depression Inventory, Rosenberg=Rosenberg Self-esteem Scale, Life Sat=Iife Satisfaction Scale 
Table 15

Summary of Rank Ordering on outcome Variables

Based on the Adjusted Postscore Cell Means

\begin{tabular}{lcccc} 
& & \multicolumn{3}{c}{ Rank } \\
Treatment X Suitability Conditions & Ist & 2nd & 3nd & 4th \\
Immediately Treated High PM & 6 & 7 & 3 & 0 \\
Immediately Treated Marginal FM & 10 & 5 & 1 & 0 \\
Delayed Treatment High PM & 0 & 2 & 6 & 5 \\
Delayed Treatment Marginal PM & 0 & 2 & 3 & 8
\end{tabular}

benefits of the immediately treated high patients were significantly greater than those of the wait list marginally suitable patients while the outcomes of the other two groups (treated marginally PM patients and delayed high FM patients) fell in between. A third variable approached significance. The three variables that conformed to the rank order predicted by hypothesis 3 were the Sexual subscale of the SAS-M, $\underline{F}(3,39)=4.4, p<.01$, the IES-A, $\underline{E}(3,36)=2.3, p<.10$, and the Beck $\underline{E}(3,39)=3.7, \underline{p} \times .05$. In general, however, the results did not support the prediction that the best outcomes would be experienced by the immediately treated, high patients.

\section{Hypothesis 4.}

Hypothesis 4 predicted that for patients in the immediate treatment condition, the high PM patients would achieve better outcomes than the manginal PM patients. The results of a series of scheffe planned comparison tests conducted on the paired means of the adjusted postscores for the immediately treated high PM versus marginal PM patients (presented in Table 14) did not support this hypothesis. The 
outcomes of these two groups of patients were comparable on all variables except the Rosenberg, $\underline{F}(3,36)=2.4$, $p<.10$ (favouring the marginal PM patients). In summary, hypothesis 4 was not supported.

\section{Hypothesis 5.}

Hypothesis 5 predicted that for patients in the control condition, the benefits for the high PM patients would surpass those of the manginal FM patients. The results of a series of Scheffé planned comparison tests conducted on the paired means of the adjusted postscores for the high PM versus marginal PM patients in the control condition (presented in Table 14) did not support this hypothesis. The outcomes of these two groups of patients were comparable on all variables. In summary, hypothesis 5 was not supported.

\section{Hypothesis 6.}

Hypothesis 6 predicted that pretherapy level of psychological mindedness (as assessed by the PMAP) would be positively related to process ratings of psychodymamic work (PWORS). To test this hypothesis, Pearson product-moment correlations were calculated between the four variables derived from the first scenario of the PMAP, and each of the five Frors ratios. As previously described, the four PMAP variables consisted of level of $\mathrm{PM}$, and the three Interpretation comprehension variables: Number of Dynamics, Speed of Dymamics, and Speed of Transference. The five PwoRs ratios consisted of the frequency of Patient Participation, Self-based Work, High Level Self-Based Work, Group-based Work, and High Level Group-Based Work. 
Table 16

Pearson correlation coefficients for PMAP with PWORS $(n=43)$

PMAP VARIABLES

PWORS RATIOS

Participation

Self-Based Work

High-level SBW

Group-Based Work

High-level GBW
Psychological Mindedness

.24

$.42 * *$

.37 *

$.28^{\mathrm{a}}$

$.34 *$
Number of Dynamics

$.41 * \star$

$.26^{\mathrm{a}}$

.10

$.43 * *$

$.40 * *$

speed of

Dymamics
Speed of Transference

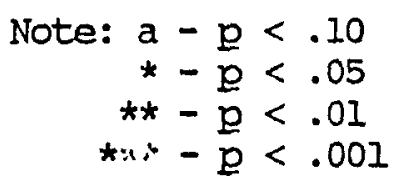

(The means and standard deviations for each PWORS ratio is presented in Appendix P). Table 16 summarizes the results of these correlations.

The correlation coefficients revealed that while PM was not significantly associated with Participation, it was associated with Self-based Work, $\underline{r}(41)=.42, \underline{p}<.01$, High-level Self-based Work, $\underline{r}(41)$ $=.37, \underline{p}<.05$, and High-level Group Based Work, $\underline{r}(41)=.34, \mathrm{p}<.05$. Hence, while psychological mindedness was not associated with speaking in the groups, it was significantly associated with engaging in psychodymamic work. Specifically, the more psychologically minded the patient, the more his/her statements reflected psychodynamic work relative to nomwork statements. Furthermore, the more psychologically minded the patient, the more he/she engaged in psychodynamic work relative to the other group members. Hypothesis 6 had been supported. 
The results of these correlation coefficients also indicated that the Interpretation comprehension variables were also related to group activity. The Interpretation comprehension variables assessed the patients' ability to understand the actor-therapist on the PMAP videotape when he interpretated the actress-patient's problem. The results indicated that the more interpreted dynamics the patient understood, (Number of Dynamics) and the ease with which he/she understood them (Speed of Dymamics) the more he/she participated in the group (Participation), and the more he/she engaged in psychodynamic work compared with other group members (Group-based Work and High-level Group-based Work). The nonsignificant correlations between the Speed of Transference and the Pwors ratios indicated that the ease with which the patient had understood the actor-therapist's interpretation of transference phenomena was not associated with group therapy behavior.

\section{Hypothesis 7 .}

The seventh hypothesis predicted that ratings of psychodynamic work would pasitively correlate with outcome. To test this hypothesis, a series of comelation coefficients were calculated for the Fwors ratios and outcome variables. The PWORS ratios were the same set that were utilized to test hypothesis 6: Patient Participation (PP), Self-based Work (SBW), High-level Self-Based Work (HISBW), Group-Based Work (GBW), and High-level Group-Based Work (HIGBW). The outcome variables consisted of the entire set of pre-post outcome variables plus the four variables that assessed the overall benefit from therapy. The entire set of outcome variables were appropriate for inclusion since this 
analysis addressed data from all. treated patients. Partial correlation coefficients were calculated for the pre-post outcome variables while Pearson correlation coefficients were calculated for the four global outcome variables. Table 17 presents the correlation coefficients between these two sets of variables.

The results of the partial correlation coefficients revealed a minimal pattern of associations between the process and pre-post outcome variables. The two significant correlation coefficie ts that were found were not interpreted since they were quite possibly due to chance.

The correlation coefficients between the global outcome ratings and the Pwors ratios were stronger. The overall Usefulness of Therapy (OUT) was positively related to participation [PP; $\underline{\underline{r}}(41)=.43, \mathrm{p}<.01$ ] and working hard relative to the other group members [GBN; $\underline{r}(41)=.45$, $\underline{p}<.01 ;$ and HLGBN; $\underline{r}(41)=.52, \underline{p}<.001]$. These associations suggest that the more the patient participated, and engaged in psychodynamic work relative to the other group members, the more the therapist rated the therapy as being useful for the patient. A similar but weaker pattern of results were revealed for the patients' global ratings of outcome. QP correlated with Participation $[\underline{I}(40)=.30, p<.10]$ and Group-based Work $[\underline{r}(40)=.30, \underline{p}<.10]$. This pattern of comrelations suggests that the more a patient participated, and engaged in psychodynamic work relative to the other group members, the more useful the he/she rated the therapy experience. This pattem was repeated for Serv Eval. A patient was more likely to positively rate the services he/she had received, if he/she had participated in the groups $[\underline{r}(37)=$ 
Table 17

Correlation coefficients for PWORS Ratios and outcome Variables

\begin{tabular}{|c|c|c|c|c|c|c|}
\hline outcome Variables & $\underline{\underline{n}}$ & PP & SBN & $\begin{array}{l}\text { PWTORS } \\
\text { HLSBW }\end{array}$ & $\begin{array}{r}\text { tios } \\
\text { GBW }\end{array}$ & HLGBW \\
\hline \multicolumn{7}{|l|}{ SAS-M } \\
\hline Work & 35 & .00 & -.04 & .02 & -.11 & -.18 \\
\hline Social & 42 & .07 & .11 & .11 & .08 & .04 \\
\hline Family & 40 & -.10 & $-.36 *$ & $-.36 *$ & -.12 & -.24 \\
\hline Partner & 14 & -.07 & -.05 & -.10 & .01 & .00 \\
\hline Children & 29 & .08 & -.25 & -.15 & .00 & -.09 \\
\hline Sexual & 42 & -.04 & -.04 & -.11 & -.05 & -.05 \\
\hline TSIA & 42 & -.04 & -.05 & -.11 & -.04 & -.12 \\
\hline TSPT & 40 & -.13 & .11 & .15 & -.13 & -.11 \\
\hline IES-I & $4 I$ & -.06 & $-.27^{\mathrm{a}}$ & $-.29^{a}$ & -.11 & -.08 \\
\hline IES-A & 41 & -.00 & -.11 & -.15 & -.06 & -.02 \\
\hline IBSP & 42 & .00 & -.09 & -.23 & -.04 & -.16 \\
\hline IDI-ER & 42 & -.03 & $-.28^{a}$ & -.20 & -.05 & -.10 \\
\hline IDI-Au & 42 & .02 & .20 & .25 & .03 & .18 \\
\hline SCI -90 & 41 & -.04 & -.14 & -.09 & -.01 & -.03 \\
\hline Beck & 42 & -.10 & .05 & .05 & -.09 & -.05 \\
\hline Rosenberg & 40 & .18 & .11 & .08 & .17 & .11 \\
\hline Iife Sat & 41 & -.05 & -.05 & .06 & -.05 & -.01 \\
\hline$\alpha u$ & 42 & $.30^{\mathrm{a}}$ & .18 & .03 & $.30^{\mathrm{a}}$ & .24 \\
\hline OUT & 43 & $.43 * *$ & .25 & .20 & $.45 * *$ & $.52 * * *$ \\
\hline Serv Eval & 39 & $.42 * *$ & .22 & .08 & $.36 *$ & $.30^{\mathrm{a}}$ \\
\hline TSPC & 41 & .18 & .10 & -.01 & .13 & .10 \\
\hline
\end{tabular}

Note: $\mathrm{a}-\mathrm{p}<.10, *-\mathrm{p}<.05, * *-\mathrm{p}<.01, * * *-\mathrm{p}<.001$ SAS-M=Modified Social Adjustment Scale; ISIA=Independently-Rated Target Severity; TSPI=Patient-Rated Target Severity, IES-I=Intrusion subscale of Impact of Event Scale, IES-A=Avoidance subscale of Impact of Event Scale, IBSP=Present Functioning subscore of Interpersonal Behavior Scale, IDI-ER=Emotional Reliance subscale of Interpersonal Dependency Inventory, IDI-Au=Autonomy subscale of Interpersonal Dependency Inventory, SCI-90=Total Score of $S C L-90$, Becks=Beck Depression Inventory, Rosenberg=Rosenberg Self-esteem Scale, Life Sat=Iife Satisfaction Scale, oup-Patient-rated overall Usefulness of Therapy, ouT-Therapist-rated overall Usefulness of Therapy, Ser Eval-service Evaulation, TSPC-Patient-rated Change in Target Severity. 
$.41, p<.01$ ] and engaged in more psychodynamic work relative to the other group members [GBN: $\underline{r}(37)=.36, \underline{p}<.05 ;$ HLGBW: $\underline{r}(37)=.30$, p<.10].

In summary, the support for hypothesis 7 was modest. There was a weak pattern of correlations revealed between the PWORS ratios and the pre-post outcome variables. A stronger pattern was found for the global ratings of outcome. Specifically, the more a patient engaged in psychodynamic work (relative to the other group members), the more useful the therapy was rated as being by both the therapist and the patient and the more satisfied the patient was with the services he/she had received.

Hypothesis 8 .

The eighth hypothesis predicted that treatment benefits would be maintained at the six-month follow-up assessment. To test this hypothesis, a series of correlated t-tests were conducted on the posttreatment and follow-up outcome scores. Data for this analysis were provided by the 36 patients who completed treatment and returned for the follow-up assessment. The outcome variables consisted of the 17 variables that were assessed pre and posttreatment plus three variables that the patient completed posttreatment.

Table 18 presents the mean for each outcome variable at the two assessment periods. The t-value associated with the change (improvement or deterioration) occurring over the follow-up period is also presented. The results indicated that for three variables, patients evidenced additional significant improvement and two variables 
Table 18

Mean and $t$-Value of

Fosttreatment to Follow-up Change on outcome Variables

for Treated Patients $(n=36)$

outcame Variables

SAS-M

Work

Social

Family of origin

Partner

Children

Sexual

\section{$\underline{n}$}

30

33

33

14

24

33

TSIA

TSPI

IES-I

IES-A

IBSP

IDI-ER

IDI-AM

SCI-90

Beck

Rosenberg

Life Sat

OUP

Serv Eval

TSPC

35

36

36

33

$\underset{\underline{M}}{\text { Posttreatment }}$

3.5

4.6

3.4

4.1

3.3

4.2

3.0

2.8

11.4

9.7

125.1

\section{5}

1.8

73.0

6.3

2.2

4.6

5.3

3.2

8.4

$\underset{\underline{M}}{\text { Follow-up }}$

3.0

4.1

3.2

3.4

3.1

4.0

2.6

2.4

8.3

8.2

.123 .3

0.56

2.4

1.9

$2.55 *$

$-0.59$

68.4

0.61

6.8

$-0.49$
2.0

0.84

4.2

1.60

5.3

0.18

3.1

9.9

0.65

$-0.92$

Note: $\mathrm{a}-\mathrm{p}>.10, *-\mathrm{p}<.05, * *-\mathrm{p}<.01, * * *-\mathrm{p}<.001$

SAS-M $=$ Modified Social Adjustment Scale; TSIA=Independently-Rated Target Severity; TSPI=Patient-Rated Target Severity, IFS-I=Intrusion subscale of Inpact of Event Scale, IFS-A=Avoidance subscale of Impact of Event Scale, IBSP=Present Functioning subscore of Interpersonal Behavior Scale, IDI-ER=Emotional Reliance subscale of Interpersonal Dependency Inventory, IDI-Au=Autonomy subscale of Interpersonal Dependency Inventory, SCL-90=Total Score of SCL-90, Beck=Beck Depression Inventory, Rosenberg=Rosenberg Self-esteem Scale, Iife Sat=Life Satisfaction Scale, oup-Patient-rated overall Usefulness of Therapy, ouT-Therapist-rated overall Usefulness of Therapy, Ser Eval-Service Evaulation, TSPC-Patient--rated Change in Target Severity. 
approached significance. These variables included the Work subscale of the SAS-M $[\underline{t}(28)=2.27, \mathrm{p}<.05]$, TSPT $[t(32)=1.89, \mathrm{p}<.10]$, IES-I $[\underline{t}(28)=3.34, \underline{p}<.01], \operatorname{IES}-\mathrm{A}[t(28)=1.72, \underline{p}<.10]$ and $\operatorname{IDI}-\operatorname{ER}[t(34)=$ 2.55, $[<.05]$. Ten of the outcame variables evidenced continued improvement although the changes were not statistically significant. one variable remained the same (OUP). None of the variables evidenced significant deterioration over the follow-up period. Four variables (IBSP, the Beck, Iife Sat and Serv Eval) evidenced a slight decrease in benefits over the period but the follow-up means were still within the normal range. In sumary, hypothesis 8 received strong support. For all outcome variables the treatment benefits were maintained over the follow-up period with five variables evidencing significant or near significant improvement.

Calculations of the clinical significance of the treatment effect were repeated for the follow-up data. These calculations addressed the possible accumulated benefits from therapy that had continued after temination. As descrihod earlier, Jacobson and Revenstorf's (1988) cut-off point and t-test methods were utilized for measures with available nomative data and the two standard deviation criterion was utilized for measures without available normative data. For those measures for which norms were available, the results indicated (Table 19) that changes evidenced by treated patients between their first and last assesment on the IFS-I, the IFS-A, the IDI-ER, and the Beck reflected clinically significant improvement. The t-tests conducted on all measures indicated that by the six-month follow-up interview, treated patients were not significartly different from the normative 
Table 19

\section{Clinical Significance of Treatment Effects At six-Month Follow-up Utilizing Normative Comparisons}

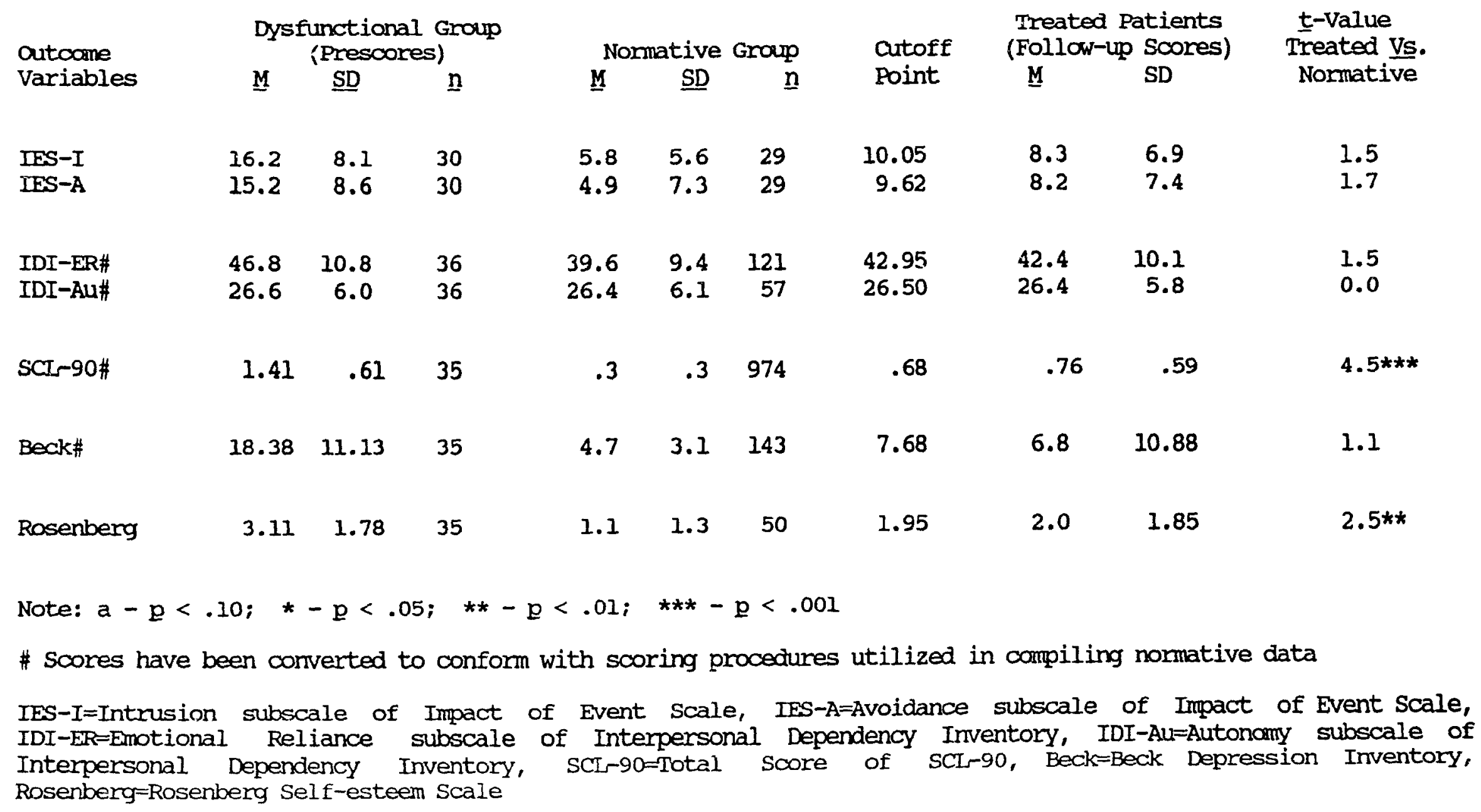


Table 20

\section{Clinical Sigmificance of Treatment Effects At Six-Month Follow-up Utilizing the Two standard Deviation criterion Since Norms were Unavailable}

\begin{tabular}{|c|c|c|c|c|c|}
\hline \multirow{2}{*}{ outcome Variables } & \multirow[b]{2}{*}{$\underline{n}$} & \multicolumn{2}{|c|}{ Prescore } & \multicolumn{2}{|c|}{ Postscore } \\
\hline & & $\underline{M}$ & $\underline{S D}$ & $\underline{M}$ & SD Units \\
\hline \multicolumn{6}{|l|}{ SAS-M } \\
\hline Work & 30 & 4.7 & 1.7 & 3.0 & .97 \\
\hline Social & 33 & 5.4 & 2.3 & 4.1 & .56 \\
\hline Family of Origin & 33 & 4.4 & 1.4 & 3.2 & .89 \\
\hline Partner & 14 & 6.2 & 2.8 & 3.4 & .99 \\
\hline Children & 24 & 4.1 & 1.7 & 3.1 & .81 \\
\hline Sexual & 33 & 7.3 & 4.1 & 4.0 & .57 \\
\hline TSIA & 30 & 4.0 & 0.6 & 2.6 & $2.2 \star$ \\
\hline TSPT & 34 & 3.9 & 0.7 & 2.4 & $2.3 *$ \\
\hline IBSP & 36 & 121.3 & 17.8 & 123.3 & -.11 \\
\hline Life Sat & 36 & 3.2 & 1.5 & 4.2 & -.66 \\
\hline
\end{tabular}

Note: * surpasses the 2 SD criterion of clinical significance

SAS-MEModified Social Adjustment Scale; TSIA=Independently-Rated Target Severity; TSPI=Patient-Rated Target Severity, IBSP=Present Functioning subscore of Interpersonal Behavior Scale, Life Sat=Life Satisfaction Scale. 
sample on the IFS-I, the IFS-A, the IDI-ER, the IDI-Au, and the Beck. Conversely, on the SCI-90 and the Rosenberg, the treated patients remained significantly more disturbed than the norm.

For those measures for which normative data were unavailable the results indicated (Table 20) that the clinical significance had increased for eight of the ten measures, compared to the posttreatment results. Changes evidenced on two of the measures, the TSIA and ISPT, now surpassed the two standard deviations criterion of clinical significance. In summary, the clinical significance of treatment benefits was stronger six months after therapy than immediately after termination (compare with Tables 10 and 11). The patients were not significantly different from ... umative samples in terms of the intrusiveness or avoidance of themes associated with the loss(es), their interpersonal autonomy and emotional reliance o.: another person, and reports of depressive symptomatology. In addition, their target objectives for entering therapy had evidenced clinically significant improvement.

\section{Additional Analyses}

\section{Therapist Effects}

It was important to investigate whether differences between the therapists had influenced the treatment effect. It was possible, for example, that the better outcomes of the inmediately treated patients were attributable to only one of the therapists. For each of the 17 outcome variables that provided pre-post data, a one-way ANCOVA 
(therapist A versus therapist B) was conducted for the immediately treated patients. For the four outcome variables that were assessed posttreatment only, independent t-tests were conducted between those patients who had been treated by therapist $A$ and those wto had been treated by therapist $B$. The results indicated only one variable evidenced a significant therapist effect. This sole finding was not interpreted given that it was quite possibly due to chance. Hence, the greater benefits of the inmediately treated patitnts relative to the control patients were not attributable to the effectiveness of only one of the therapists.

Evidence for a therapist effect was also investigated with all treated patients. Of the 21 outcome variables subjected to analysis, only one provided evidence for a therapist effect. This sole finding was also quite possibly due to chance. In summary, differences between therapists were not found to have significantly confounded the treatment effect.

\section{Therapist Ratings}

Analyses which involved therapist ratings addressed the clinical validation of the research instruments. First, the associations between therapist ratings of patient characteristics and the PMAP variables were investigated. The associations between the therapist ratings of therapy process and the FWORS ratios were also explored. The predictive ability (in terms of outcome) of the therapist ratings compared to the research instruments was also investigated.

First, the relationship between clinical impressions of the patient 
Table 21

Correlation coefficients for Therapist-rated Patient Characteristics and the PMAP ( $n=42)$

PMAP Variables Therapist-rated Patient char
Psychological Response to
Mindedness Interpretation Mindedness Interpretation Iikeability

Psychological Mindedness

$.30 *$

$.46 * *$

$.46 * \star$

$.37 *$

$$
.40 *
$$

$.46 * *$

$.50 * *$

$.41 * *$ $.30^{\mathrm{a}}$

$.36 *$

$.34 *$

$.42 \star *$

Note: $a-p<.10 ; *-p<.05 ; \quad * *-p<.01 ; \quad * * *-p<.001$

and the PMAP ratings was determined. Clinical impressions of the patient's Psychological Mindedness, Response to Interpretation and Iikeability were provided at sessions 4, 8, and 12. Pearson correlation coefficients were calculated for the mean rating of each therapist-rated variable and the PMAP variables. The correlation coefficients are presented in Table 21. Therapist ratings of Psychological Mindedness were significantly correlated with the PMAP assessment of $\mathrm{BM}[\underline{I}(40)=.30, \underline{p} \times .05]$. The therapist ratings of Response to Interpretation were significantly correlated with the PMAP assessment of IC [Number of Dynamics: $\underline{r}(40)=.46, p<.01$; Speed of Dynamics: $\underline{r}(40)=.50, \underline{p}<.01$; Speed of Transference: $\underline{r}(40)=.41$, p<.01]. The strength of these associations offered clinical val idation of the PMAP variables.

The results also indicated significant correlations between the 
therapist ratings of Iikeability and the PMAP variables, especially the IC variables [Number of Dynamics: $\underline{r}(40)=.36, \underline{p}<.05$; Speed of Dymamics: $\underline{r}(40)=.34, p<.05 ;$ Speed of Transference: $\underline{r}(40)=.42$, p<.01]. This pattern of result's indicates that the therapist liked those patients who understood the concepts of conflictual dynamic components and transference and who were psychologically minded $[\underline{r}(40)$ $.30, \mathrm{p}<.10)$.

To investigate the relationship between the therapist-rated impressions of the patient and outcome, partial and Pearson correlation coefficients were calculated. The results of these correlations (Table 22) indicated a weak pattern of associations between the clinical impressions and the pre-post outcome variables. Only two significant correlations were revealed. They were not interpreted since they are quite possibly due to chance. A stronger pattern was found for the global outcome measures, especially for the therapist's global ratings of therapy outcome (OUT). Specifically, the more psychologically minded $[\underline{r}(41)=.58, \underline{p}<.001]$, responsive to interpretations $[\underline{r}(41)=$ .51, $p<.001]$, and likeable $[I(41)=.57, p<.001]$ the patient was, the more useful the therapist raied the therapy. A similar, but weaker pattern was revealed for the patient's global ratings of therapy outcome (OUP). Hence, therapist ratings of these three patient dimensions were positively related to both the patient's and the therapist's global appraisals of therapy outoome.

In sumary, therapist ratings of patient characteristics provided after each third of therapy were found to be minimally related to therapy outcome. corversely, positive global appraisals of therapy 
Table 22

Correlation coefficients for
Therapist-rated Patient characteristics and outcome

Therapist-rated Patient Characteristics Psychological Response to

outcome Variables

n Mindedness

Interpretation Iikeability

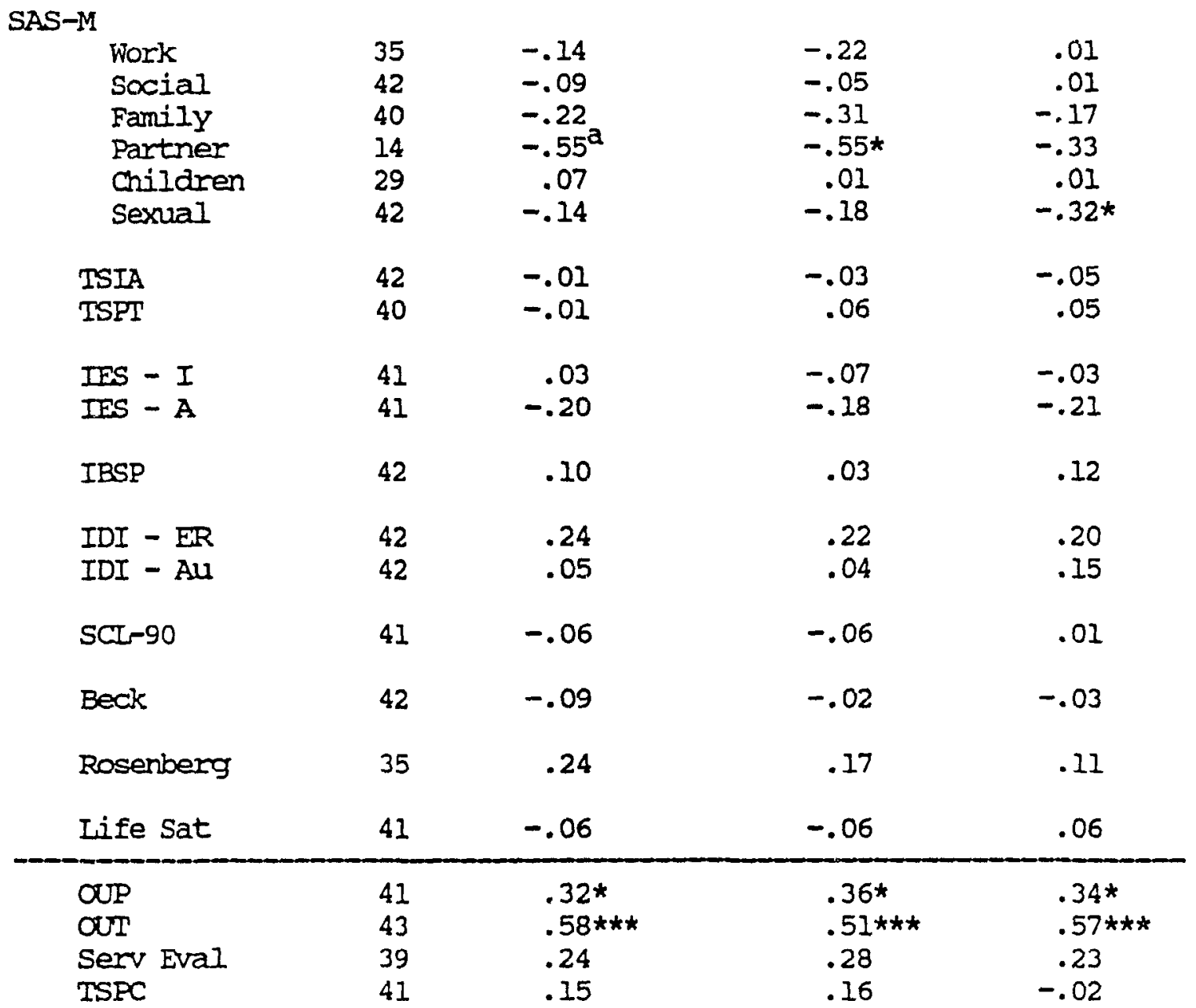

Note: $\mathrm{a}-\mathrm{p}<.10, *-\mathrm{p}<.05, * \star-\mathrm{p}<.01, * \star \star-\mathrm{p}<.001$

SAS-M Modified Social Adjustment Scale; TSIA=Independently-Rated Target Severity; TSPT=Patient-Rated Target Severity, IES-I=Intrusion subscale of Impact of Event Scale, IES-A=Avoidance subscale of Impact of Event Scale, IBSP=Present Functioning subscore of Interpersonal Behavior Scale, IDI-ER=Emotional Reliance subscale of Interpersonal Dependency Inventory, IDI-Au=Autonomy subscale of Interpersonal Dependency Inventory, SCI-90=Iotal Score of SCI-90, Beck=Beck Depression Inventory, Rosenberg=Rosenberg self-esteem Scale, Iife Sat=Life Satisfaction Scale, oup-Patient-rated Overall Usefulness of Therapy, OT-Therapist-rated Overall Usefulness of Therapy, Ser Eval-Service Evaulation, TSPC-Patient-rated Change in Target Severity. 
Table 23

\section{Correlation coefficients for \\ Therapist-rated Process \\ and Fwors variables $(n-43)$}

PWORS ratios

Therapist Process Ratings Participation Work

Participation

Self-based Work

High-level Self-based Work

Group-based Work

High-level Group-based Work

$$
\begin{aligned}
& .86 \star \star \star \\
& .52 \star \star \star \\
& .29^{a}
\end{aligned}
$$$$
.87 \star \star \star
$$$$
.78 \star \star \star
$$

$.53 \star \star \star$

$.57 \star \star \star$

$.44 \star \star$

$.56 * \star \star$

$.61 \star \star \star$

Note: $\mathrm{a}-\mathrm{p}<.10$

$\star-\mathrm{p}<.05$

$\star \star-\mathrm{p}<.01$

$\star * *-\mathrm{p}<.001$

outcome were significantly related to high psychological mindedness, being responsive to interpretations and being likeable.

A final series of analyses addressed the relationship between clinical ratings of process and the pwors. Clinical ratings of process consisted of (the mean of) therapist postsessional ratings of Participation and work. As previously described, the pwors ratios consisted of Participation, Self-Based Work, High-level Self-based Work, Group Based Work, and High-level Group-based Work. Pearson correlation coefficients were calculated for the two sets of variables. The results (Table 23) indicated that the therapist rated process ratings were significantly related to process ratings provided by the PWORS. Specifically, therapist ratings of Participation significantly comelated with four of the Pwors variables 
[Participation: $\underline{r}(41)=.86, \underline{p}<.001$; Self-based Work: $\underline{r}(41)=.52$, p<.001; Group-based Work: $\underline{r}(4.1)=.87, \underline{p} . .001 ;$ High-level Group-based Work: $\underline{r}(41)=.78, \underline{p}<.001]$. The fifth FwoRs variable, High-level

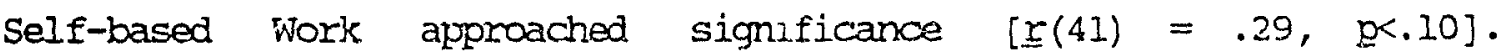
Therapist ratings of work significantly correlated with the FWoRS work variables: Self-based Work $-\underline{I}(41)=.57, \underline{1}<.001$; High-level Self-based Work $-\underline{r}(41)=.44, \underline{p}<.01$; Group-based Work $-\underline{r}(41)=.56, \underline{p}<.001$, and High-level Group-based Work $-\underline{r}(41)=.61$, $\underline{p}<.001$. The strength of these associations offered clinical validation of the Pwors.

It was important to detemine whether the therapist ratings of process were better predictors of outccme than the PWORS ratings. This issue was addressed by calculating Partial and pearson correlation coefficients between the therapist ratings of process and the outcome variables. The results (Table 24) revealed a generally weak pattern of associations between the clinical ratings and the pre-post outcome variables with no significant associations being found. The relationship was stronger for the global outcome ratings. Specifically, therapist ratings of Participation significantly comelated with the overall [isefulness of Therapy as rated by both the patient $[\underline{\underline{r}}(40)=.30, \underline{p} \times .05]$ and by the therapist $[\underline{r}(41)=.58$, p<.001]. Participation was also significantly correlated with Serv Eval $[\underline{\underline{r}}(37)=.38, \underline{p} .05]$. In addition, therapist ratings of work significantly correlated with the therapist's appraisal of the overall Usefulness of Therapy $[\underline{r}(41)=.51, \underline{p}<.001]$. This pattern of associations suggests that with the exception of glabal appraisals of therapy usefulness, therapist impressions of process were not strongly 
Table 24

Correlation coefficients for Therapist-rated Process and autoome Variables

\begin{tabular}{|c|c|c|c|}
\hline & & Therapist Process & Ratings \\
\hline outcome Variables & $\underline{n}$ & Participation & Work \\
\hline SAS-M & & & \\
\hline Work & 35 & .05 & -.10 \\
\hline Social & 42 & .07 & -.07 \\
\hline Family of origin & 40 & -.18 & -.15 \\
\hline Partner & 14 & .05 & .12 \\
\hline children & 29 & .10 & -.06 \\
\hline Sexual & 42 & -.06 & -.06 \\
\hline TSIA & 42 & .03 & -.03 \\
\hline TSPT & 40 & -.03 & .11 \\
\hline IES - I & 41 & -.10 & -.16 \\
\hline IES - $A$ & 41 & .03 & -.05 \\
\hline IBSP & 42 & -.13 & -.03 \\
\hline $\begin{array}{r}I D I \text { - ER } \\
-A U\end{array}$ & 42 & .04 & .13 \\
\hline$-A \mu$ & 42 & .03 & .04 \\
\hline$S C L-90$ & 41 & .00 & .10 \\
\hline Beck & 42 & -.09 & .02 \\
\hline Rosenberg & 40 & .25 & $.30^{\mathrm{a}}$ \\
\hline Iife Sat & 41 & -.03 & -.16 \\
\hline oup & 42 & $.30 \star$ & .24 \\
\hline orr & 43 & $.58 * \star \star$ & $.51 * \star \star *$ \\
\hline Serv Eval & 39 & $.38 \star$ & .11 \\
\hline TSCP & 41 & .20 & .00 \\
\hline
\end{tabular}

Note: $\mathrm{a}-\mathrm{p}<.10, \star-\mathrm{p}<.05, \star \star-\mathrm{p}<.01, \star \star \star-\mathrm{p}<.001$

SAS-M=Modified Social Adjustment Scale; TSIA=Independently-Rated Target Severity; TSPI=Patient-Rated Target Severity, IES-I=Intrusion subscale

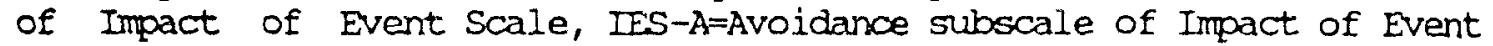
Scale, IBSP=Presert Functioning subscone of Interpersonal Behavior Scale, IDI-ER=Emotional Reliance subscale of Interpersonal Dependency Inventory, IDI-Au=Autononty subscale of Interpersonal Dependency Inventory, SCI-90=Total Soore of SCI-90, Beck=Beck Depression Irventory, Rosenberg=Rosenberg Self-esteem Scale, Life Sat=Life Satisfaction Scale, oup-patient-rated Overall Usefulness of Therapy, aT-Therapist-rated overall Usefulness of Therapy, Ser Eval-Service Evaulation, ISPC-Patient-rated change in Target Severity. 
related to therapy outcome. A comparison of the pattem of associations between the clinical impressions of therapy process and outcome (Table 24) versus the ProRs ratings and outcome (Table 17) revealed that the predictive power of the therapist is very similar to that of the PwORS. 


\section{Disarsian}

The discussion is organized around three major areas: the outcome (section one), therapeutic process (section two) and patient suitability (section three) for short-term psychoanalytically oriented group psychotherapy as conducted and irvestigated in this study. The first section is divided into two subsections: the treatment effect and the clinical significance of the treatment effect. The section addressing the therapeutic process of the groups consists of five subsections. The first one describes the possible curative factors inherent in STG. This description is followed by a discussion of whether STG therapeutic process constituted psychoanalytic work. The thind subsection concerns the degree to which psychodynamic work was found to be a curative factor in the groups. To provide a context for evaluating the success of STG therapeutic process, a brief review of the success of alternative group treatments evoking other therapeutic processes with loss patients constitutes the fourth subsection. The final subsection in the discussion of therapeutic process concerns patient attrition. The thind section evaluates the success of psychological mindedness as a selection variable. The predictive ability of the Interpretation comprehension variables is also discussed in this section. This is followed by the clinical implications of the relationships between psychological mindedness and STG process and outcome. Specifically, alternative strategies in the treatment of marginally psychologically minded loss patients are presented. A fourth section considers results irvolving the therapists in the 
study. The discussion of the major findings is followed by a presentation of limitations associated with the present study, a sunmary of the strengths and implications of the study and a presentation of the original contribution of this research.

\section{The outcome of STG}

\section{The Treatment Effect}

The results indicated that patients who were inmediately treated with STG improved significantly more on outcome measures than their matched counterparts on the wait list (Hypothesis 1). In addition to evidencing better outcome relative to the control patients, the benefits evidenced by the immediately treated patients were found to represent (statistically) significant improvements. The benefits included areas of interpersonal functioning, psychiatric symptomatology, self-esteem, and general life satisfaction. Conversely, the control patients evidenced slight improvement over the wait period, deteriorating in some cases. The mean effect size for all outcome variables utilized in this study (.79) was comparable to the mean effect size of all psychotherapy (.85) derived from a meta-analysis of all outcome studies (Smith, Glass, \& Miller 1980). The range of the effect size in this study suggests that STG was particularly effective for some areas of disturbance (e.g. self-esteem) but not for others (e.g. occupational functioning). These results offer stronger support for the efficacy of STG than the previous empirical. studies of STG conducted by Budman et al., 1984, Piper et 
al., 1984 and Lapointe and Rinm, 1980 (reviewed in the Introduction). The effectiveness of STG was found to be less impressive for - patients who proceeded from the wait list through the treatment phase. The pretherapy to posttherapy outoome scores indicated beneficial treatment effects, but only a few reached criterla of statistical significance. The diminished efficacy of STG for the delayed treatment patients relative to the immodiately treated patients may have been cue to the rather small mumber of patients (17) available for this analysis. The lower $n$ resulted in reduced power for the own-control analyses. Conceptual explanations involve possible interfering effects of the wait period. For example, they could have interpretod the delay as confinmation of their feelings of worthlessness. This speculation is supported by the finding that self-esteem was the one variable which evidenced significant deterioration over the wait period. It is also possible that the delay evoked anger with respect to experiencing further deprivation. This heightened anger could have been displaced from the research staff onto the group therapist creating obstacles in the formation of a cohesive working group. Finally, the improvements evidenced over the delay period, although slight, could have made their lives more tolerable. Hence, patients' motivation to take risks and change might have diminished as their complaints became less debilitating. This finding suggests that there is an optimal time for offering ary patient therapy and that a wait period may inadvertently interfere with their motivation for and their ability to benefit from subsequent therapy. 
Quantitatively Determined Clinical simificance of outcome

The determination of clinical significance based on the posttherapy data was less impressive than the statistical tests of treatment efficacy. For those measures where normative data were available, the results indicated that the average treated patient's level of functioning remained within the disturbed (pretherapy) range. similarly, for those measures where normative data were not available, only two scores approached a change of two standard deviation units. However, those two scores representod changes in the patient's target objectives. In addition, the mean postscore on the Autonomy subscale of the Interpersonal Dependency Inventory was not significantly different from the normal population. On the Impact of Event Scale, patients' reports of avoidance and intrusion of themes associated with the loss(es) were not significantly different from reports of a nomal population 13 months after a traumatic event.

Eefore considering the implications of these findings, it should be noted that the quantitative determination of the clinical meaningfulness of a treatment's outoome remains a controversial issue. For example, Saunders, Howard, and Newman (1988) presented the philosophical objection chat the utilization of norms to determine treatment efficacy is to equate confomity or social adjustment with the goal of psychotherapy. They also pointed out the arbitrariness and cultural relativism inherent in utilizing normative criteria to Indicate treatment effectiveness. Related to this issue, Strupp and Hadley (1977) have pointed out that social adjustment is one aspect of therapy outcome with the patient and therapist's subjective evaluations 
representing two equally important but not necessarily congruent aspects. For example, the patient's evaluation of therapy outcome may be based on his/her self-contentment while the therapist's evaluation may reflect his/her theoretical biases such as the maturity of the prevalent defense mechanisms utilized. Other objections raised in the literature included the argument that change resulting from treatment may be interesting and meaningful despite falling short of normalcy (Hollon \& Flick, 1988). Hence, reviewers of Jacobson and Revenstorf's (1988) proposed method of calculating clinical significance have advocated the use of relative criteria of functioning rather than demanding absolute normalcy (Hollon \& Flick, 1988; Nietzel \& Trull, 1988).

Despite the controversy and obstacles regarding the methods and meaning of quantitatively determined clinical significance, the issue is a crucial one and was addressed in the present study. There were obstacles to the quantitative determination of the clinical meaningfulness of changes evidenced in the present study. For example, there were problems presented by the utilization of multiple measures. This raised the issue of how to evaluate the differential effect of STG on areas of functioning. There was also the issue of whether to include areas of functioning that had not been identified as problematic by the patients.

The major obstacle, however, was the absence of normative data for many of the outcome variables. The few measures for which normative data were available were utilized despite doubts concerning the appropriatess of the normative sample. These doubts were based on the 
theoretical issue of whether any mourning process was ever "resolved." This issue involves debating whether patients who had experienced multiple losses could ever become "normal." It became obvious tirat an evaluation of clinical significance had to be considrared within the context of the patient population. For exarple, a treatment for schizophrenics can be effective (in some areas) despite its failure to render the patients within the normal range of functioning (Nietzel \& Trull, 1988). In the case of the present study, therefore, the evaluation of clinical significance begins with a theoretical understanding of the pathology exhibited by this population. This understanding is based on a synthesis of the writings of various psychoanalytic and object relations theorists in addition to the present author's understanding of themes and confilicts that emerged in the groups throughout the study. (The following description also serves as the basis for a future discussion regarding the therapeutic process and curative factors of the groups.) While the psychcunalytic and object relations theoretical understandings of loss primaril; concern bereavement, the issues and processes involved in mouring any loss, be it by death or separation (divorce), are considered to be universal. The nature of the presenting complaints may differ, however, depending on features of the loss.

The Nature and Etiology of Pathological Grief. The task of mourning involves accepting the reality that the relationship to the object (significant person) no longer exists. Hence, the emotional investment in or attachment to the lost abject has to be withdrawn. 
Since this process of detachment is gradual, the existence of the lost abject needs to be contimued in the mind until the process is complete (Freud, 1917). The subject must ficst form an introject (internalized representation) of the lost person and then interact and negotiate with this introject until the object belongs to the past where nothing more can be expected from it (Tahka, 1984).

When a person resists accepting the significance or the impact of the loss such that the self-limiting process of mourning is arrested, pathological grief is said to be ocourring. While pathological grief may appear as a prolonged mourning process it may also appear as a grief reaction whose onset has been umusually delayed. The actual features of normal mouming and pathological grief may be identical with one notable distinction. As Freud (1917) first noted, while normal mourning involves an attitude of loss of an object, pathological grief (which he termed melancholia) involves an attitude of loss of self. Hence, a loss of self-esteem is a characteristic feature of pathological grief.

By understanding the development of self-esteem one begins to understand the nature and etiology of pathological grief. positive self-esteem is closely linked with the primary caretaker's (usually mother) loving and concerned accessible presence, and with the pride with which she invests her child (Tyson, 1983). If a loss, separation, or disappointment occurs priur to the establishment of internalized ideals, values, and self-worth, the subject will remain dependent on the presence of extemal abjects to perform the praising and punishing functions. Ultimately, a person's vulnerability to pathological grief 
corresponds with the degree to which the psychic structure (personality) is dependent on the presence of an extermal object. Freud (1914) referred to these types of object relationships as narcissistic, while Tahka (1984) referred to them as prestructual. These types of abject relat onships fail to engender autonomy from the sustaining abject or a constant sense of self and self-worth. Klein attributed them to a failure to adaquately negotiate the depressive position (Klein, 1948). In addition to reflecting arrested development, these types of object relationships are considered to be fraught with hostility. Hence, hostile dependency, inadaquate reparation, or ambivalence characterizes the relationships of those people who are predisposed to pathological grief (Tahka, 1984; Tyson, 1983; Lerner \& Lemer, 1987; Klein, 1948; Horowitz, Wilner, Marmar \& Krupnick, 1980)

Given that the lost object served: unction for the person, the loss must be resisted (denied) until the function has been replaced. The person may deny the loss in the hope of completing the developmental process of separation and autonomy with the now introjected object (Lemer \& Lemer, 1987). The denial of the loss imvolves maladaptive consequences, however, which may bring these people to the attention of mental health professionals. The following section describes several consequences of pathological grief which constituted the conflicts and presenting complaints of this study's patient population.

The ambivalence which characterized their relationships gave rise to the fear that their hostility towards the abject was responsible for 
the loss. At the very least, they were afraid that the object left because of defects in themselves, or because they were not lovable enough. These fears may have been supported by tautological arguments such as: 'since I failed to prevent the loss, I am weak, incompetent, and uncaring' (Horowitz et al, 1980). In cases where the loss was preventable (deaths related to car accidents, cigarette smoking, alcohol abuse and all separations/divorces) failure to rescue the object was tantamount to being responsible for the loss. The feelings of responsibility and guilt with respect to the loss may have reflected, therefore, the unconscious fear that their anger had magically killed or barished the lost abject. The patients may have denied the potency of their hostility by presenting as helpless and depressed. It is noteworthy that ambivalence with respect to the lost object was typically denied. The lost object was either idealized (particularly in the case of death) or vilified (particularly in the case of separation, divorce). Hence, while the "negative" side of the ambivalent feelings was commonly denied after a death, it was the "positive" side of the ambivalent feelings that was commonly denied after a separation/divorce.

A typical reaction to the loss of the object was the attempt to replace himpher wi hh another person. Such replacements often lead to transient relationships which resulted in yet more losses. Additional losses served to confirm the patients' fears that they were despicable and responsible for former losses. If the substituted object relationship was maintained the expectations and anger that were then displaced onto this second person often resulted in marital or 
interpersonal conflicts. Relationships which offered the patients the opportunity to learn to perform these functions for themselves and hence, complete the developmental sequence towards autonomy were resisted. This resistance seemed to reflect their need to punish themselves for the former loss. In addition it seemed that new relationships threatened to dissolve even the memory of the fomer relationship. The threat of absolute dissolution rendered the patient feeling disloyal and guilty while confirming their fears that they did have the power to destroy another.

The confusion wrought by loss(es) often brought fears of repetition if another relationship was attempted. Hence, the patients remained isolated despite feeling lonely and incomplete. They needed yet feared another relationship which made intimacy, with anyone, a struggle. often patients attempted to substitute alcohol, food, or work for the original murturing (lost) abject. These substitutions tended to only perpetuate the depression, shame, low self-esteem, interpersonal difficulties as well as creating and/or exacerbating somatic complaints.

The diverse symptoms and conflicts that characterized the presenting complaints of this patient population were understood as manifestations and consequences of pathological grief. In this respect it is perhaps not surprising that two-thirds of the patients who had warranted an axis II diagnosis had received dependent personality disorder. The criteria for this personality disorder are: "passively allows others to assume responsibility ... because of an inability to function; subordinates own needs to those of person on whom he or she 
depends ...; sees self as helpless" (DSM-III; American Psychiatric Association, 1980, p.326). Theoretically, at the base of these difficulties were the patients' inability to tolerate the existence of ambivalent teelings towards the lost abject, the hostile dependent nature of their relationships and their developmental failure to separate. In addition to addressing the presenting complaints of these patients, therefore, the therapeutic goal included the exploration of the common uncorscious features and their connection with the patients' conscious difficulties. The nature and etiology of pathological grief forms, therefore, the context within which one considers the clinical significance of the results.

The CIinical Significance of STG. The results indicated that there were only a few areas where STG had changed the patients in a clinically meaningful way. These zreas represented, however, the personalized problens for which they had sougint treatment (the target objectives). By the end of treatment, the patients were as autonomous interpersonally as the normal population. In addition, a roduction in the disturbing impact of the loss(es) had been accomplished. This reduction had represented the focus of STG. These results are interpreted with restrained optimism regarding the efficacy of STG. While the groups, by their very nature were not expected to explore the entire range of conflicts existing in the patients, a thorough exploration and understanding of the particular conflicts associated with loss had been postulated to benefit the patients by offering them an important model for understanding subsequent and concurrent problems 
and conflicts in their lives. The treatment focused on the issue of loss and the nature of their interpersonal interactions (i.e. their lack of autonomy interpersonally). While the results of the statistical analyses had determined that the treatment had sionificantily affected this and other aspects of functioning, the impact. was not, as yet, clinically significant. Many areas of finctioning remained problematic. The groups did not deliver a "quick fix." Nevertheless, the patients' and therapist's goals for treatment had been reached. The process of recovery had been set in motion. considering the rather primitive (i.e. prestructural, depressive, dependent, narcissistic) nature of the patients' personality structure and/or interpersonal relationships, and the imvestment of time by the patients and therapist, the impact of STG is considered to be clinically significant and no small accomplistment. The working through process would have to contime beyond the termination of therapy. Theoretically, gains in the targetted areas of functioning would cont imue and eventually influence the patients' entire lives.

These conclusions are similar to those of Budman et al. (1984):

Such groups may not "cure" prablems in living nor dramatically alter character structure, if indeed such changes are ever possible under any circumstances. They may, however, provide patients with the opportunity to begin to change aspects of their lives about which they feel dissatisfaction or demoralization. Furthermore, even moderate change may carry with it the seeds of mastery and hope. These factors in turn may facilitate more effective problem-solving when future stresses emerge (p.601).

Analyses of the follow-up data reaffinmed the (statistically determined) treatment effectiveness (Hypothesis 8). Treatment gains 
had been maintained six months after the termination of therapy. In some areas the patients had continued to improve. Calculations of the clinical significance of these benefits revealed that improvement in the patients' goals for therapy (target objectives) had now surpassed the two st undard deviations criterion of significance. The patients were also as autonomous and emotionally reliant as the normal population. Their experience of depressive symptomatology was now indistinguishable from the norm. Finally, the dismptive impact of the loss(es) was now below the reports of a nomal population thirteen months after a traumatic event. In summary, the benefits of treatment had continued after therapy had ended becoming more pronounced in some areas. The areas which evidenced contimued improvement represented those same areas considered to be particularly problematic for this patient population. The follow-up results indicated that the benefits of treatment for these areas of difficulty were now clinically significant.

\section{The Therapeutic Process of STG}

\section{curative Factors in STG}

The efficacy of STG may have reflected a particularly good match between a treatment approach (STG) and a patient population (loss patients). Hence, the success of STG may have reflected the resonance between the groups' therapeutic approach and the patients' concerns and conflicts. The following section summarizes the manner in which the therapeutic process is believed to have addressed typical processes and 
thiemes that evolved in the groups.

With the first session, conflicts of intimacy immediately came to the fore as patients struggled to coalese into a working group, cisclose personal information and overoome their fear of vulnerability. Their vulnerability was particularly elicited by the knowledge that involvement would be followed by loss (temination) after only eleven more sessions. Predictably, patients attempted to comfort themselves (and avoid intimacy with each other) by substituting the therapist for their lost gratifying object. Inis was reflected in patients' demards for advice, structure and explanations from the therapist. Despite the substantzal activity level of the therapist, inevitably, he/she was perceived as being passive, judgemental and deliberately depriving them of the "secret formula" necessary to resolve their difficulties. This mispercoption of the therapist may have been due to the the type of therapist activity which was predominantly interpretive and clarifying rather than directive and supportive. Since the patients were not obtainung what they felt they needed from the therapist, it was as if they were receiving nothing at all. The therapist interpreted the frustrations and disappointments witis him/her and the patients' fear of the same. By interpreting the "unspeakable" the patients were indirectly encouraged by the therapist to express their "negative" feelings. The therapist's acceptance of these feelings were seen to promote an atmosphere of safety.

The homogeneous composition of the groups facilitated this anxiety-laden beginning stage of the groups as members recognized and identif.ed with the similar compiaints and concerns of each other. The 
curative benefits from identifying with similarities of other patients has been referred to by Yalom (1985) as universality. Simultaneously, the group modality allowed patients to develop multiple relationships which gratified some of their dependency needs, thus substituting for the lost object. It is noteworthy that many theoretical papers have compared any group to the "good mother". Elbirlik (1983), for example, postulated that group members will evoke memories of early figures and pathological attachments. Hence, there is a parallel between mourning and group process since both situations require the patient to abandon a familiar way of interacting and attempt something different and frightening (Elbirljk, 1983). In this study, it was perthaps the combination of a homogeneous group who shared the same conflicts and fears with respect to relationships, who offered support, reassurance and acceptance to each other, and a therapist who encouraged the patients to express their ambivalence with respect to him/her that enabled the patients to begin to explore their ambivalence with respect to past depriving objects, who typically included the lost object(s).

The homogeneous composition of the group was therapeutic in another, ironic way. As every member sought to have the group satisfy his/her dependency needs, each member was the recipient of other members' demands. By experiencing first hand, therefore, the demanding and overwhelming aspects of other patients' interactions, the patients began to realize their own contribution to failed relationships. The possibility of homogeneous groups becoming volatile due to each member's negative aspects being reflected in other members has been documented in the literature (Stava \& Bednar, 1979; Furst, 1975). For 
this population, the expression of hostility, especially in reaction to their own maladaptive patterns of interaction, was considered therapeutic.

While the groups were homogeneous with respect to loss, the kind of losses were heterogeneous. The majority of the patients had experienced multiple losses, both by death and by separation. The combination of both types of losses in the same group had a serendipitous effect. It was noted that patients who focused on a death tended to deny anger towards the lost object. Conversely, patients who focused on a separation/divorce tended to deny sadness towards the lost object. By having both types of losses in the same group, and indeed, often within the same patient, the expression of all types of emotion was facilitated in the groups.

Another unexpected therapeutic factor soon appeared in the groups. This was the group's reaction to the dropout. Initially, absent members tended to be protected and defended. This was interpreted as the patients' fears of "speaking ill of the dead." Eventually, the group began to acknowledge experiencing a variety of feelings towards the "empty chair." These feelings included concern, sadness, rejection, regret, guilt and the less spontaneously acknowledged affects of anger, envy or relief. The group's ambivalent feelings toward the lost member were interpreted as being reminiscent of similarly ambivalent feelings toward past losses in their lives. In addition, acknowledging feelings of envy towards the departed member led to members' appreciation of their ambivalence towards the group and other relationships in their lives. Thus, patients' reactions to the 
loss in the here-and-now life of the group were interpreted by the therapist as being similar to their reactions to past losses. While dropouts are generally considered to have a demoralizing effect on psychotherapy groups, in this study, their departure tended to consolidate the bond between the remaining members. Perhaps once the patients' (worst) fears of abandomment had been realized they felt somewhat freer to become involved in the group. This tendency of dropouts to facilitate cohesion among remaining members has been noted in the literature by Lothstein (1978).

The time-limit of the groups was seen as being particularly therapeutic for these loss patients. Temination represented a unique opportunity for patients to explore and re-experience their idiosyncratic reactions to loss which had created so much difficulty for them in the past. By understanding the relationship between their current feelings with respect to termination and their past reactions to losses, they could experiment with different and more adaptive reactions to loss. Hence, the loss of the group could also serve as a rehearsal for future losses.

Initially, for these patients who were afraid of losing control of their aggressive impulses, and who feared the destructive ability of their dependency needs, the clearly defined boundaries imposed an external control which tended to contribute to the safety of the group. Eventually, the members commonly expressed the wish that the group contimue past its predetermined termination date. Similarly, members wished to socialize with each other between and beyond the sessions. These wishes were interpretod as attempts to deny the 
inevitability of the loss of the group and the related fears of isolation and abandonment which were reminiscent of their conflict with respect to past losses. Similarly, frustrations that the group had not resolved all the patients' problems and that the termination was premature were interpreted as being reminiscent of past, premature 10sses. Discomfort with the variablity among members' satisfaction with the group was interpreted as the patients' fear of indivicuality and survivor guilt. Survivor guilt referred to the tendency of the patients to feel guilty living while others close to them had died.

In addition to these shared themes in reaction to termination, each member's idiosyncratic reaction to the loss of the group was interpreted as being a familiar pattern originating in the past. The inherent difficulties consequent of these patterns were identified. These idiosyncratic reactions to the loss of the group included: devaluing the group experience to avoid the sadness; becoming helpiess or in crisis to prolong the group sessions; talking of dropping out to avoid a more direct expression of anger. Examples of experimenting with different, more adaptive ways of addressing loss included confronting or supporting another nember rather than regretting yet another missed opporturity. The ease with which patients were more accepting of ambivalent feelings with respect to the therapist, the group members, the group experience, the end of the group, and the memory of past lost objects were speculated to indicate the diminished impact of past (and future) losses.

Support for the aforementioned explanations of the groups' efficacy with this population can be found in the clinical literature. It has 
been argued, for example, that psychoanalytically oriented group therapy is the treatment of choice for patients who: are overdependent and overdemanding, tend to withdraw from relationships, become involved in multiple transient relationships, fear the amipotence of their anger, fear and avold the dyadic situation of indivictual therapy, experience difficulties of adjustment and functioning in social situations, and complain of a life situation of loneliness, dreariness and a lack of stimulation (Neumann \& Gaoni, 1974; Grunebaum \& Kates, 1977). For these patients the group can represent a corrective emotjonal experience (Grunebaum \& Kates, 1977). This term signifies the opportunity for patients to experiment with different ways of interacting within an emotionally intense yet safe enviroment. In addition, patients' dependency needs are more likely to be gratified and tolerated in a group where they are divided among many people (Neumann \& Gaoni, 1974).

There are remarkabla similarities between patients believed to be particularly suited to group therapy and the characteristics of the loss patients. While group therapy may be the treatment of choice for loss patients, the success of the short-tem modality remains impressive. As noted earlier, however, the time-1imitud nature of the groups seemed to resonate with the conflicts inherent in loss. In this respect, the success of STG is consistent with Mann's (1973) model of time-limited indivicual psychotherapy, (described in the Introcuction). Patients were offered the opportunity to understand and utilize their reactions to the loss of the group as a model for beginning to work through the unresolved conflicts that were associated 
with their previous losses. In addition to the the time limit of the group, factors believed to have facilitated the efficacy of STG included the group modality, the homogeneous composition, the therapist focus on here-and-now interpersonal interactions and the linking of the same with past relationships, especially the lost relationship, and the exploration of member-member and therapist transference phenomena. In conclusion, the interaction between the STG therapeutic process and the patient population is believed to have been responsible for the efficacy of STG.

\section{The Integrity of the Psychoanalytic Process}

A major issue addressed by the present study was the feasability of conducting psychoanalytic work on an on-going basis in the STC format. As reviewed in the Introduction, psychoanalytic work has been defined as entailing a regressive process, a technical process and a progressive process (Bienvem et al., 1986). In response to the anxiety of the beginning stages of the groux, it has previously been mentioned that the patients attempted to substitute the therapist for the lost gratifying abject. Hence, they seemed to regress to a dependent mode of functioning where they attempted to procure the saretaking function from the therapist. This dependent mode of functioning was manifested in the patients' demands for advice, structure, explanations and encouragement from the therapist. When the therapist did not collude with their dependency, he/she was perceived as passive, judgemental, and deliberately depriving of their wishes. Their perceptions of the therapist seemed to elicit further regression 
as the patierits projected their anger onto the therapist accusing him/her of being rejecting and punitive. Hence, it was concluded that the groups had elicited the regressive process of psychoanalytic work.

Clinical appraisals of the groups confimed that the therapists had succeeded in carrying out an analytic technical process. They had maintained a neutral position and interpreted transference and unconscious conflicts as they manifested themselves in the here-and-now events of the group. The integrity of the technical process was empirically investigated by monitoring the frequency and type of therapist interventions, utilizing the PwORS. This investigation revealed that the average number of therapist interventions per session was 17 which represented 16 percent of all verbal productions in the group. Sixty-three percent of all theraplst interventions were interpretations with one-fifth involving mulitiple conflictual dynamic components. The empirical evidence supported the clinical appraisals of a successfully conducted analytic technical process.

The third component of psychoanalytic work, the progressive process was clinically reflected in the patients' efforts to understand and work with the therapist's interpretations. 'Ine patients' ability to work within the analytic approach was empirically investigated by monitoring the quantity and quality of psychodynamic work evidencerl in the sessions, utilizing the pwors. This investigation revealed that almost half of all patient speaking time involved the identification of dynamic componesits. Statements that focused on multiple, conflictual dymamic components represented slightly over one-fifth of all their speaking tirne. The empirical evidence supportat the clinical 
appraisals of a progressive process in the groups. In addition, the amount of time that the patients engaged in psychodynamic work was comparable to that of the therapist. The work levels of STG patients were also comparable with those of patients engaged in long-term psychoanalytically oriented group therapy. Piper, Doan, Edwards and Jones (1979) utilized a modified version of the Hill Interaction Matrix to irvestigate the work levels in their groups. They reported that Iong-term group patients engaged in work approximately 53 percent of the time. In conclusion, given the evidence that regressive, technical and progressive processes had occurred in STG, the transition of psychoanalytic group psychotherapy to the short-term format was considered a success.

\section{Psychodynamic Work as a Curative Factor in STG}

It had been hypothesized that the more a patient engaged in the therapeutic process, as reflected in his/her levels of psychodynamic work, the more he/she would benefit from the group (Hypothesis 7). The patient's engagement in psychodynamic work (as rated by the Frors) represented, therefore, the curative factor that was empirically irvestigated in the present study. The results indicated a generally weak pattern of direct associations between levels of work and pre-post measures of outcome. The two significant correlations that were found were attributed to chance considering the mumber of variables involved in the correlation matrix.

The lack of significant direct relationships between levels of psychodynamic work and the majority of the outcome variables may be 
partially che to the lack of variability and range in outcome scores. The patients improved regardless of their work level in the group. Those patients who failed to benefit from the group dropped out. Hence, they did not contribute to the data set. Another explanation involves the limitations of the FWCRS measure. The system did not monitor the incidence of work occurring covertly within the session, or work that occurred between sessions. In addition, the system failed to evaluate the emotional irpact or integration of the work being verbalized within the sessions. These other aspects of work may have played a crucial mle in the relationship between work and outcome in the groups. Other explanations for the rather meagre relationships found between work and outcome in the present study involve the complexity of therapeutic process. It is possible, for example, that the relationship between work and outoome differs between patients. Some patients may need to connect a conflict with an undesirable consequence only once in order to facilitate change while other patients may need to ponder the same connection several times before it resonates with them and results in change. It is also possible that other therapeutic conditions or factors inherent in the groups mediated or influenced the relationship between work and outcome. Related to this issue, is the point raised by stiles (1988) concerning the possibility that patients may require different amounts and combinations of therapeutic factors in order to obtain positive outcomes. It is evident that the recipe for outcome remains obscure with either the ingredients and/or the quantities of each ingredient requiring further irvestigation. 


\section{Success of Alternative Treatments for Ioss Patients}

The present study did not address the efficacy of STG relative to other therapeutic approaches or modalities for this population. There are reports in the literature which attest to the efficacy of other treatment approaches with a similar population, the conjugally bereft. For example, Lieberman and Videka-Sheman (1986) evaluated the efficacy of self-help groups with this population. They reported improved functioning for widows who had elected to participate in a seif-help group program for one year compared to those who had declined. These authors also reported that the 25 percent of their population who had reported exposure to adjunct psychotherapy did not. significantly benefit from that exposure. These latter findings are rather ambiguous, however, since 42 percent of the psychotherapy contacts were of less than six sessions duration and the authors relied on the participants' report of the type and usefulness of the contact. The study does attest, however, to the usefulness of self-help groups with a population believed to be highly susceptible to the development of psychological/emotional problems.

Barrett (1978) compared the outcomes of 70 widows who had been recruited from the community and then randomly assigned to one of three group treatments or a (delayed treatment) control group. The treatments consisted of a self-help group, a confidant group or a consciousness-raising group. The two groups conducted within each condition met for seven weekly sessions of two hours duration. The format of the self-help group consisted of members sharing solutions to practical concerns while the therapist acted as a facilitator. The 
confidant group involved each member pairing with one other person to engage in intimacy-building exercises while the therapist moved from pair to pair "as needed." A discussion around a list of possible sex role topics considered to be of particular relevance to widows constituted the fornat of the consciousness-raising group. Barrett reported that all groups (including the control group) evidenced substantial changes. On two of the twelve cutcome measures, the treatment groups were somewhat more effective than the control group. There were no significant differences between the outcomes of the three treatment approaches. There was a trend, however, in favour of the consciousness-raising group. The author attributed the effectiveness of this group approach to the facilitation of expressed anger.

The outcome studity conducted by Marmar, Horowitz, Weiss, Wilner and Kaltreider (1988) involved a clinical population. These researchers compared the efficacy of individual versus group treatment of conjugal bereavement. In that study, 61 widows were randomly assigned to twelve sessions of either psychodynamically oriented individual therapy (STI) or a self-help group (SHG). The individual therapy sessions were one hour in length while the group treatment sessions were one and one-half hours in length. Their results indicated that patients in both conditions improved over the course of the study and that the two treatments were equally effective. As with the present study, these authors also reported that the treatment was very effective for some areas of disturbance (symptom reduction) but not for others (interpersonal and cocupational functioning). They attributed this latter finding to "the long-term process of role transition 
necessitated by the death of a spouse" (p.208).

In summary, there have been few controlled outcome studies that have investigated the efficacy of group treatment approaches with loss patients. Intervention has often represented secondary prevention strategies. Self-help graps have been the preferred treatment approach. While their general helpfulness has been concluded, controlled outcome studies of the efficacy of group treatments for conjugally bereaved women have offered ambiguous results. No form of treatment for this patient population has been found to be superior to another.

\section{Attrition}

A number of patients dropped out at various times throughout the present study. The dropouts represent a subgroup of patients with whom the STG therapeutic process did not resonate. Dropping out was not found to be related to condition assignment. This finding should ease ethical concerns over utilizing a delayed treatment control group paradigm. Despite the counterarguments that it is unethical to provide treatment without verifying its efficacy, delaying treatment for a randomly selected portion of patients is always difficult. condition assignment may not have affected attrition in this study since the patient was informed that his place in the subsequent therapy group was reserved. He/she was informed of the starting date of the group. Patients vere also made aware that if their circumstances deteriorated, they could recontact their intake therapist and procure support or arrange an alternate treatment plan. 
The dropout rate curing the treatment phase was 28 percent. Io be considered a dropout, the patient had to attend at least one session but not complete treatment. This study's dropout figure was actually at the average level of usual dropout rates for group therapy. Lothstein (1978) for example, quoted a dropout rate for long-term group therapy that ranged from 25 to 50 percent. com srsely, Piper et al. (1984) reported a dropout rate of 17 percent from their short-term groups. Those authors found no differences between those patients who dropped out and those who remained in their groups which were conducted according to traditional psychoanalytic principles. The controlled outcome study conducted by Budman et al. (1984) did not experience any dropouts among their 36 participants. The authors attributed this impressive finding to the success of their (single, one and one-half hours) screening workshop. (Four patients were excluded from their study clue to their presentation during the workshop.) In an earlier paper, however, Bucman et al. (1980) reported a dropout rate of 17 percent from 22 short-term groups that they had been monitoring. As described in the Introcuction, Budman and his colleagues conducted psychodynamically oriented short-term groups that were based on a model of adult development. They characterized their dropouts from the groups as distant and insensitive towards others, distrustful of the group experience but at the same time holding unrealistic hopeful expectations of the grorp. These authors implied that their dropouts lacked a basic liking and trust for people adding that to succeed in SIG patients needed "to be able to 'speak the same language' or at least be able to hear the words" (Budman et al., 1980, p.15). 
While Budman et al. (1980) were referring to interpersonal comunication by the phrase "speak the same language", the drrpouts in the present study where similarly unable to 'speak the same language' as the other members. Dropping out of treatment in the present study was found to be significantly related to the paitent's level of psychological mindedness (PM). While 62 percent of marginal PM patients dropped out only six percent of high PM patients failed to complete therapy. The differential attrition rates between the patients indicated that the majority of the marginal PM patients could not tolerate the groups. It is highly probable, therefore, that the marginal PM patients dropped out of therapy because they felt confused and dissatisfied with the psychoanalytically oriented therapy process.

The following description typifies the experience of marginal PM patients who dropped out of STG. Often they came late to the first session with their attendance contimuing to be erratic thereafter, with absenteeism, lateness or early departures. Typically, they offered reasonable excuses such us work schedules or ilinesses as being responsible for their poor attendance while denying any ambivalence with respect to their membership in the groups. When in attendance, they often sat silently throughout the sessions. When invited by another group member to participate, they rarely added to the discussion stating that they agreed with most of what had already been said. When they did participate in the group discussion, they complained about their physical condition, the incompetency of the medical profession to correctly treat them, their doubt regarding the connection between their current concerns and "scmething that happened 
years ago and that happens to everyone." Their confusion with the therapist role was revealed by such statemants as "when I call a plumber I expect hin to know how to fix things." Hence, these patients interpreted the therapist's lack of advice and direction as reflecting incompetence. They could not explure the possibility that their resistance to the therapist was being exacerbated by associating himpher with the medical doctors who had failed to save their lost one. For them, the group was experienced as "the blind leading the blind."

The speculation that the marginal PM patients felt lost in the groups is supported by the fact that two thirds of the dropouts left the group churing the early sessions. The early sessions have been reported elsewhere as being the period of high risk for any group therapy (Klein \& Carroll, 1986; Budman et al., 1980). Mackenzie and Iivesley (1983) speculated that early dropouts in their short--tem therapy groups failed to engage due to fears of being found unaoseptable by the other members. Given the level of guilt and "unacceptable" anger experienced by loss patients, it is perhaps not surprising that since they were unable to understand the connection between their symptoms and their feelings with respect to the loss(es), their feelings of helplessness and confusion escalated to the point where they dropped out.

It is equally possible that these patients had interpreted their Ieferral to the groups as yet anotner abanaiorment by either their general practitioner or by their intake therapist. Typically these patients had jeen referred by their general practitioner who had 
suspected that their chronic complaints of vague aches and pains, imitability and insomia were actually manifestations of a low level type of depression or dysthymia. Although these patients were adamant that their symptoms were no "all in their head" there were many possible reasons for their accepting referral to the groups. For example, they had to agree that talking about the loss(es) still brought tears to their eyes or that the onset of their aches and pains had coincided with a loss or an anniversary of a loss. They also may have realized that what they had attempted thus far had been ineffectual and that the mmerous medical tests that they had undergone had failed to reveal any physical ailment. Their failure to engage with the other group members may have represented, therefore, their anger with respect to being referred to a treatment they considered to be second best (indivichal therapy being their first choice). Their difficulty overcoming their apprehension in the group probably represented their difsiculty identifying with the highly psychologically minded patients, not understanding the therapist's role or interventions and their prohibitive fear of intimacy.

The one-third of the dropouts who left the group during the terminating sessions were considered to have had difficulty with the loss of the group. While the psychoanalytic process may or may not have resonated with these patients, they nevertheless had persisted with the group, However, when these patients were confronted with yet another loss, they fajled to understand and/or work with the connection between their reaction to the loss of the group and their reactions to past losses. Faced with the repetition of loss, these patients coped 
with their feelings of helplessness and anger by abandoning the group rather than waiting to be abandoned. It was as if the group's termination pre-empted any encuring positive evaluation of the experience. Mackenzie and Livesley (1983) have similarly postulated that dropping out late in treatment reflects difficulties resolving the existential problem of one's essential isolation and responsibility for oneself.

Marmar et al. (1988) also cited the painful nature of the termination phase as precipitating dropping out for their patients who were already sensitized by a recent major loss (i.e. death of spouse). In the Marmar et al. (1988) study, the authors reported that while 29 percent of patients in the short-term individual therapy condition dropped out, 77 percent of patients in the mutual self-help group terminated prematurely. To explain the differential attrition rates between the two conditions, the authors cited variable individual responses to stressful events which acted to disrupt the formation of early cohesion. Specifically, these authors speculated that the dropouts were "threatened by witnessing powerful abreactive expressions" of emotions that they, themselves, were trying to disavow (p.208). It is unlikely, however, that it was the nature of the patients difficulties (i.e. bereavement) that solely precipitated the high attrition rate in that study. For example, Yalom and vinogradov (1988) reported only a five percent dropout rate in their paper describing the techniques and themes of their support groups for the bereft. It is conceivable that the particularly high attrition rate in the Marmar er al. (1988) study is attributable to their use of 
nonclinicians as the groups' leaders. It is possible that they were unable to manage the debilitating anxiety of those early sessions. The low dropout rate reported by Yalom and Vinogradov (1988) may be attributable to the fact that experienced group psychotherapists led the groups. It may also be attributable, in part, to the selection of a non-clinical pcpulation. Hence the members may have been less vilnerable to the abreactive experiences of other members.

In summary, level of psychological mindedness was found to be strongly related to dropping out in the present study. The nature of the patient population and the psychodymamic therapeutic approach may have contributed to the relationship betweer M and attrition. These results imply that marginally psychologically minded patients should be referred to another form of therapy. Marginal PM patients are usually considered "a better prospect for chemotherapy (or behavioral or supportive therapy) than for unoovering therapy" (Abramowitz \& Abramowitz, 1974, p.610) The decision to refer marginal PM patients to altermative treatment in the future imvlves considering psychological mindedness as a selection variable. The next section discusses this issue.

III. Patient Suitability for STG

\section{Psychological Mindedness as a Selection Variable}

As previously mentioned, psychological nindedness was found to be stongly related to remaining or dropping out of therapy. For those patients who remained in the groups, the results indicated that 
psychological mindedness was related to therapy process. Specifically, while $\mathrm{M}$ was not related to talkativeness, it was associated with engaging in psychodynamic work (Hyputhesis 6). Psychological mindedness was not found to be a prognostic variable since it was not strongly related to "spontaneous" remission rates of patients on the wait list (Hypothesis 5). Similarly, it was not found to be a curative factor since it was not strongly related to general improvement (Hypothesis 2). To be considered a selection variable, however, psychological mirdedness had to have influenced the response to treatment.

The influence of psychological mindedness on outcome was generally weak. The finding that was revealed was unexpected (Hypothesis 3). The results indicated that those marginal BM patients who compieted the group benefited as much as or more than the high PM patients. Explanations of this finding are tentative due to the small rumber of patients involved. Rather than high levels of psychological mindedness being a selection criterion, the results suggest that it is the marginal PM patients who should be offered STG. Given that the majority of the marginal PM patierits could not tolerate the groups, it is interesting to speculate how this "high risk, high gain" population did benefit from the grouns.

While treated patients improved regardless of the level of psychological. mindedness, it is unlikely that the marginal pM patients benefited fxcm the same process as the high PM patients. While psychodynamic work and the psychnanalytically oriented process may have been the high PM patients' route to success, it is likely that the 
marginal PM patients benefited frm other curative aspects present in the groups. Yalom (1985) has identified several curative factors that he believed occur in any therapy group. They included universality, corrective recapitulation of the primary family group, instillation of hope, and the development of socializing techniques. Hence, the high and marginal PM patients may have followed different routes but arrived at the same destination. This explanation is consistent with the minimal influence of psychological mindedness on outcome (Hypotheses 2, 4, and 5), the aforementioned strong pattern of associations between PM and psychodynamic work (Hypothesis 6) and the weak pattern of associations between psychodynamic work and outcome (Hypothesis 7).

In support of this explantion is the study reported by connelly and Piper (1989) wherein patients who engaged in different therapeutic processes were still able to derive benefit from psychodynamically oriented long-term group therapy. They speculated, therefore, that the patients engaged in a process which was most suited to and most beneficial for them. However, they also found that the therapists rated patients who had engaged in low levels of psychodynamically oriented group process as having benefited more from insight oriented therapy than their counterparts who had engaged in high levels. This finding suggests that even though the patients failed to engage in high levels of psychodynamically oriented therapy process, their exposure to this process was, nevertheless the curative factor in their treatment, according to the therapist.

It is possible that the psychoanalytically oriented process as evidenced in STG helped marginal PM patients by offering them ways of 
viewing problems that was most novel for them. Being exposed to a philosophy which espoused responsibility and hence, control for one's lot in life (the verbalizations of the high group members and the therapist interventions) may have combatted the patients' feelings of helplessness and hopelessness, encouraging them that they could change their lives. Iearning through exposure to other people's behavior is called vicarious learning. This phencmena has been also conceptualized as a curative factor in group psychotherapy (Yalom, 1985). In addition, it was identified as a curative factor by impatients engaged in short-term graups (Brabender, Albrecht, Sillitti, Cooper \& Kramer, 1983). The view that marginal psychological minded patients can benefit fram psychootyamically oriented therapy has been espoused in the clinical literature (Neumann and Gaoni, 1974).

To summarize, the majority of manginal PM patients in the groups aropped out. Those who did persist in the groups benefited from the experience. Psychological mindedness was not particularly related to patients' benefit from therapy. Treated patients improved regardless of the levei of psychological mindedness. It is unlikely, however, that the marginal FM patients berefited from the same process as the high PM patients. They engaged in less psychodynamic work than the high PM patients. Factors that may have differentiated the marginal PM patients who remained in the group from those who left and that subsequentlv, allowed them to benefit from the group may have been their ability to benefit vicariously from the process of the high $\mathrm{MM}$ members and/or other nonspecific factors occurring in the groups. The marginal FM patients who remained in the group long enough to take 
advantage of its curative factors, might have been better able to interact interpersonally than those who dropped out. Their interpersonal ability seemed to have compensated for their lack of psychological mindedness. The importance of interpersonal factors for remaining in STG has befan documented in the literature (Budman et al., 1980).

While psychological mindedness did not prove itself as a seiection variable, the results of the present study do have implications for the preparation and/or selection of marginal PM patients for future STG loss groups. In addition to the patients' level of psychological mindedness, their ability to understand therapist interpretations of conflict between dymamic components and of transference phenomena was also assessed by the PMAP. The predictive ability of these variables is discussed in the next subsection.

\section{Interpretation comprehension as a Predictor of STG Process and outcome}

similar to psychological mindedness, the pattern of associations between the Interpretation comprehension variables and the pre-post measures of outcome was generally weak. In terms of therapy process, the pattern of associations between the Interpretation comprehension variables and the PWORS process variables was stronger. Patients who were receptive to interpretations of conflictua' dynamic components participated more in the groups and engaged in more psychodynamic work than other members. These associations suggest that they felt more comfortable in the groups, talking and working regardless of the other members. They were not inhibited by the other members. 
These process measures and the both types of the Interpretation comprehension variables (dymamics and transference) were found to be related to therapist and patient ratings of the overall usefulness of therapy and patient satisfaction with services received. Hence, the valie ascribed to the STG experience, by both the therapist and patient, varied depending on the patient's involvement in the group and receptivity to the fundamental psychoanalytic concepts of conflictual dynamic components and transference phonomena. In sumary, the Interpretation Comprehension variables were minimally related to outcome but modestly related to process measures and global impressions of therapy outcome. This pattern of associations suggests that receptivity to therapist interpretations may influence patient satisfaction with a therapy technique that emphasizes interpretation. Psychological mindedness remained, however, the more powerful. predictor variable given its association with attrition and process. The implications of these associations are discussed in the next subsection.

\section{Treating Marginally Psychologically Minded Loss Patients}

Two strategies for the future treatment of marginal PM loss patients involve offering an alternative therapeutic approach or modifying STG. The first strategy assumes that marginal PM patients would do equally well or better in another form of treatment but that they would be less likely to abandon this alterate treatment. To lower the risk of dropping out, they could be offered a therapy that was more directive, structured and supportive in form. By maintaining the group 
format, the patients could contimue to benefit from the curative factors inherent in all groups.

It is possible, however, that vicarious learning resulting from their exposure to novel ways of viewing their problems (i.e. the psychoanalytic process), was a salient curative factor for this type of patient. If this was the case, then they should contimue to be offered STG. Certain modification would need to be implemented, however, in order to reduce the high dropout rate amoing the marginal PM patients. Iothstein (1978) suggested that dropouts could be prevented by reducing the ambiguity of the early sessions. Rather than utilizing the early sessions, Budman et al. (1984) utilizated a single pretherapy workshop session. As previously mentioned, those authors attributed their zero dropout rate from their controlled outcome study to this procecture. Similarly, Piper et al. (1984) have found that pretraining or pretherapy preparation sessions do successfully lower dropout rates in Iong-term group therapy. With respect to the format of a pretherapy workshop, Piper et al. (1982) reported that it was the experiential (interpersonal) aspects of the pretraining exercises rather than the cognitive information conveyed that influenced the subsequent dropout rate. Consistent with this finding, Iothstein (7.978) reported that the dropouts he surveyed did not attribute their departure from group to a lack of preparation. Hence, a pretraining session that facilitated interpersonal interactions between group members, conducted in a structured and supportive way could be implemented with future loss patients. The high PM patients would also attend this pretraining session since recomposing groups after the pretraining experience has 
been found to dismpt the positive influences of pretraining on remaining (Connelly \& Piper, 1989).

A third consideration raised by the results of this stui. involves composition strategies for STG. It is possible that the marginal PM patients who remained in the groups were able to benefit only after the other marginal FM patients dropped out leaving the group composed of a majority of high FM patients. With the departure of most of the marginal FM patients, the psychcanalytic process would not be detoured. Hence, the marginal PM patient may need to be surrounded by rather than merely exposed to highly psychologically minded patients to benefit. This speculation is supported by the work of connelly and Piper (1989). They found that patients scoring high on pretherapy levels of work (high-level patients) evidenced higher therapy levels of work when they were in groups composed of other high-level patients than with a mixture of high-level and low-level patients. This phenomenon can be called a context effect. Hence, the future compositior. of STG may need to vary the ratio of high to marginal PM patients to optimize the contert eftut.

There is clearly a need to research the aforementioned strategies for modifying the future treatment of marginal PM patients. First, there needs to be a comparative study that investigates whether or not marginal FM patients benefit more from a supportive therapeutic approach than from a psychoanalytically oriented one. A comparative outcome study would contime the investigation of possible matches between a patient population and a treatment approach. As the design of the comparative study would involve referring manginal PM patients 
to STG, pretraining procedures would need to be implemented to increase the probability that they would remain in the groups (in order to compare their outcomes with their counterparts in the support group). Referring high PI patients to a supportive treatment involves ethical considerations. It is possible that the high PM patients would not benefit as much from a supportive approach as they would from STG. Abramowitz and Abramowitz (1974) reported, for example, that highly insightful college students benefited more from insight-oriented therapy than from a supportive approach. In addition, they reported that the highly insightful students did no better than their minimally insightful counterparts when both experienced the supportive therapy. It may be necessary, therefore, to include a cross-over phase where each group of patients experienced both types of therapeutic intervention.

IV. Therapist Effects.

There was little evidence for a therapist effect on outcome. Patients improved regardless of the particular therapist conducting the group. Treatment effects were not, therefore, significantly confounded by differences between the therapists. Therapist ratings of patient characteristics (psychological mindedness, response to interpretation, likeability), and processs variables (participation, work) offered clinical validation of the research instruments developed to assess patient predictor variables (PMAP) and therapy process (PWORS). The therapist ratings were not superior to the research instruments in 
terms of predicting outoame despite the fact that they were based on the patients' presentation throughout the course of therapy. conversely, the PMAP ratings were based on a thirty-mimute pretherapy administration of the device.

Therapist ratings of patient characteristics were minimally related to pre-post outcome measures but positively related to both patient and therapist appraisals of the overall usefulness of therapy. This finding suggests that the more the therapist perceived the process resonating with the patient (in terms of his/her display of psychological mindedness and response to interpretations), the more useful the therapist judged the therapy to have been for the patient. The patient's agreement that the therapy was useful validated the therapist's appraisals.

\section{Iimitations and suggestions for Future Research}

The methodological limitations associated with the current study have been identified in previous sections. They are sumarized in this section.

The major weakness of the study was the few immediately treated marginally psychologically minded patients who actually completed treatment. The high rate of attrition among this cell in the study severely recluced the power of the tests of interaction effects. The high dropout rate also prevented the investigation of the three-way and four-way interaction effects between treatment, suitability, therapist, and groups. It is conceivable that by focusing on the effects of the two manipulated independent variables, more complex interactions were 
masked. Generalizing from results based on the data of so few patients must be done cautiously due to possibility that the marginal PM patient; in this study are not representative of typical marginal PM patients. Future research must devise strategies to better retain these patients. These strategies may involve pretherapy exercises that focus on increasing cohesion or modifications to the therapeutic technique that address the anxiety of the initial sessions.

A second limitation concermed the criteria for psychodymamic work included in the PWORS. The system did not monitor the incidence of work occuring covertly within the session, or work that occurred between sessions. In addition, the system failed to evaluate the emotional impact or integration of the work being verbalized within the sessions. It is possible that these other components of therapeutic process contribute to the relationship between psychodynamic work and outcome. Perhaps the development of a measure of emotional involvement or engagement could be devised or ut: $\because d$ to complement the Fwors. Another limitation of the PWORS was its $r$ : ince on frequency data. It is possible that the impact of psycinociymic work is independent of frequency and that an insight or connection need only be realized once to have impact. In addition to utilizing adjunct or more comprehensive measures of work, future measures could reflect complex and interactive models of therapeutic process. In this way, future researchers may discover variables that moderate the relationship between psychodynamic work and therapy outcome.

With respect to the outcome battery, this study primarily relied on the patient's self-report. The inclusion of a measure completed by someone who knew the patient might have enhanced the objectivity of the 
outcome assessment. Since the goal of psychodynamic therapy is to foster insight, a measure of each patient's understanding of his/her idiosyncratic confiicts would have complemented this study's outcome battery. It would be useful if future research endeavours addressed the construction of a measure of psychoctynamic change.

This study did not address the efficacy of STG compared to alternate treatment approaches. It is possible that supportive interventions may be more efficacious with the marginal PM patients. Future research studies would do well to compare the benefits of STG with other therapies.

Generalizing the efficacy of STG as practised in the current study to wher populations should be done cautiously. It is possible, that the benefits evidenced in this study represented a particularly good match between a patient population and a therapeutic approach. Future studies could investigate the efficacy of SIG with another equaliy suitable population. A comparative study of the efficacy of STG with two different populations would also be informative.

\section{Strengths and Implications}

This study represents the largest controlled, clinical trial investigation of STr to date. Its major strengths, therefore, include the number of groups involved, the random assigmment of patients to the inmediate treatment versus the control condition, repeated administration of the outcome battery, the utilization of experienced therapists, the selection of a clinical population and the inclusion of a six-month follow-up period. The fact that the outcome battery 
included measures representing a variety of areas of functioning and different sources of evaluation was also a strength. Requiring each therapist to conduct equivalent mubers of groups in each condition controlled for therapist effects across conditions which increased the likelihood that the treatment effects were due to the independent variable (Dies, 1979). The major clinical implication of this study is that the enthusiasm of mary clinicians for STG has been empirically substantiated. The practice of STG constitutes a valid treatment option for loss patients.

The deveiopment of the PMAP addressed the recommendation that research instruments reflect congruence between operational and conceptual definitions of relevant variables (Bednar \& Moeschl, 1981). The PMAP is an efficient, easily administered measure of two clinically relevant variables. Its psychometric properties were initially established in a pilot study and the results of this study supported its reliability and validity with a clinical population. The strong association between level of FM as assessed by the PMAP and attrition from therapy suggests that it is possible to identify probable dropouts before the first group session. This identification has implications for the selection and/or preparation procedures for manginal PM patients with respect to this type of therapy. Finding that marginal FM patients who persisted with STG derived benefits from the experience implies that other patient characteristics (e.g. interpersonal abilities) may be able to compensate for psychological mindedness in these groups.

The strong associations between the PMAP variables and 
psychodynamic work in the groups suggest that it is possible to identify patients who are amenable to working within this orientation during the assessment process. This identification has implications for the selection ard/or composition procedures for psychodynamicilly oriented therapy groups. The rather weak pattern of direct relationships between the PMAP variables and the outcome indires suggests that there are patient variables and/or process variables mediating between the predictor and outcome variables.

The development of the pwors reflects a congruence between operational and conceptual definitions of a relevant clinical concept, psychodynamic work. The criteria of work utilized in the PwORS are based on groum phenomena, for example, the impact of one member on another. Hence, the work variables reflect primary and unique dimensions of group treatments (Bednar \& Moeschl, 1981). The PWORS proved itself as a reliable and valid measure of a component of therapy process. Psychodynamic work was significantly related to pretherapy measurements of patient psychological mindedness and modestly related to global appraisals of outcome. Utilizing a process analysis systern rather than relying on therapist reports of process was a more objective measure of the therapeutic process of STG. The possibility of experimenter bias was significantly reduced.

This study investigated the interaction between patient characteristics, therapy process and outcome. This investigation acknowledged the warning that particular treatment methodologies may interact with specific patient characteristics to determine psychotherapeutic outcome (Parloff \& Dies, 1977). The manipulation of 
a patient characteristic addressed the necessity of matching a treatment modality with suitable patients (Beutler, 1979).

The two familiar criticisms of group poychotherapy research were addressed by the current study. The first criticism involves the lack of integration between research and theory (Kaul \& Bednar, 1986). The therapy mamual constructed for STG attempted to specify and describe the components of the therapeutic process. There was also an attempt to identify the possible curative factors inherent in STG. This identification involved a conceptual discussion of the rature and etiology of this patient population and the manner in which this may have been addressed by therapy.

The second criticism typically levied against group psychotherapy research involves its lack of clinical relevance (Dies, 1983a). This study has addressed the issue of clinical relevance by discussing the clinical significance of the results. In addition, an attempt was made to integrate the process and outcome of STG such that clinicians could implement the tiserapeutic techniques in their own practice. The study of short-term therapy represented a particularly relevant choice of treatment given the necessity of developing cost-effective treatments (Parloff \& Dies, 1977). The choice of loss patients as this study's population represented the decision to address a prevalent patiert population. Death and separation are parts of life. As every change can be considered a loss, the relevance of the treatment of pathological reactions to loss cannot be overstated. 


\section{Original contribution}

The present study represents the largest controlled, clinical trial investigation of STG to date and contributes to the literature concerning the efficacy of this treatment modality. The successful transition of psychoanalytically oriented group therapy process to the short-term format has been empirically demonstrated. The development of the PMAP represents an efficient, standardized, reliable and valid measure of two cilinically relevant variables that is based on behavioral referents. In particular, the operationalization of the variable, psychological mindedness, represents an important contribution to the mental health field. This study is the only one in the STG literature to date that investigated the interaction between a clinically relevant patient characteristic (psychological mindedness), therapeutic process (psychodynamic work), and outcome. Investigations that address the complex interactions between these three aspects of psychotherapy have been recommended by reviewers of the group psychotherapy research literature (Kaul \& Bednar, 1986; Parloff \& Dies; 1977; Woods \& Melnick, 1979). 


\section{Bibliography}

Alexander, F., \& French, T. (1946). Psychoanalytic therapy: Principles and applications. New York: Ronald Press.

Abramowitz, S.I., \& Abramowitz, C.V. (1974). Psychological-mindedness and benefit from insight-oriented group therapy, Archives of General Psychiatry, 30, 610-615.

American Psychiatric Association. (1980). Diagnostic and statistical manual of mental disorders (3rd ed.). Washington, D.C.: Author.

Ammons, R.B., \& Ammons, C.H. (1962). The quick Test: Provisionai manual, Psychological Reports.

Appelbaum, S.A. (1973). Psychological mindedness: Word, concept, and essence. International Journal of Psycho-Analysis, 54, 35-46.

Appelbaum, S.A. (1977). The anatomy of change. New York: Plenum Press.

Bachrach, H.M. (1980). Analyzability: A clinical-research perspective. Psychoanalysis and Contempory Thought, 3 , 85-116.

Baekeland, F., \& Iundwall, L. (1975). Dropping out of treatment: A critical review. Psychological Bulletin, 82, 738-783.

Baer, D.M. (1988). If you know why you're changing a behavior, you'll know when you've changed it enough. Behavioral Assessment, 10, 219-223.

Barrett, C.J. (1978). Effectiveness of widows' groups in facilitating change. Journal of Consulting and Clinical Psycholocy, 46, 20-31.

Bauer, G.P., \& Kobos, J.C. (1987). Brief therapy: Short term psychcdymamic intervention. New Jersey, London: Jason Aronson, Inc.

Beck, A.T., \& Beamesderfer, A. (1974). Assessment of depression: The depression inventory. In P. Pichot (Ed.), Psychological measurement in psychophannacology: Modern problems in pharmacopsychiatry (Vol. 7, pp. 151-169). Paris: Karger, Basel.

Beck, A.T., \& Steer, R.A. (1987). Beck Depression Inventory manual. The Psychological Corporation, Harcourt Brace Jovanovich Inc., New York.

Bednar, R.L , \& Kaul, T.J. (1979). Experiential group research: What never happened! Journal of Applied Behavioral Science, 15, 311-319.

Bednar, R.I., \& Moeschl, M.J. (1981). Conceptual and methodological considerations in the evaluation of group psychotherapies. In $P$. Mareynolds (ed.). Advances in psychological assessment (vol. 5) (pp. 393-423) San Francisco: Jossey-Bass. 
Bergin, A.E., \& Lambert, M.J. (1978). The evaluation of therapeutic outcomes. In S.L. Garfield and A.E. Bergin (Eds.), Handbook of psychotherapy and behavior change. New York: John Wiley \& Sons.

Beutler, L.E. (1979). Towara specific psychological therapies for specific conditions. Joumal of Consulting and Clinical Psychology, 47, 882-892.

Bienvemu, J.P., Piper, W.E., Debbane, E.G., \& de Carufel, F.I. (1986). On the concept of psychoanalytic work. American Journal of Psychotherapy, 40, 277-289.

Bilodeau, C.B., \& Hackett, T.P. (1971). Issues raised in a group setting by patients recovering from myocandial infarction. American Journal of Psychiatry, 128, 73-78.

Blanchard, E.B., \& Schwarz, S.P. (1988). Clinically significant changes in behavioral medicine. Behavioral Assessment, 10, 171-188.

Bloch, S. (1979). Assessment of patients for psychotherapy. British Journal of Psychiatry, 135, 193-208.

Bloch, S., \& Crouch, E. (1985). Therapeutic factors in group psychotherapy. Oxford: Oxford University Press.

Bock, R.D., \& Haggard, E.A. (1968). The use of multivariate analysis of variance in behavioral research. In D. Whitla (Ed.), Handbook of measurement in echucation, psychology and sociology, Reading, Mass.: Addison-Wesley.

Bond, M., Gardner, S.T., Christian, J \& Sigal, J.J. (1983). Empirical study of self-rated defense styles. Archives of General Psychiatry, $40,333-338$ 。

Brabender, V., Albrecht, E., Sillitti, J., Cooper, J., \& Kramer, E. (1983). A study of curative factors in short-term group psychotherapy. Hospital and Community Psychiatry, 34, 643-644.

Budman, S.H. (Ed.). (1981a). Forms of brief therapy. New York: The Guilford press.

Buchman, S.H. (1981b). Significant treatment factors in short-term group psychotherapy. Group, 5, 25-31.

Budman, S.H., \& Bernett, M.J. (1983). In H. Kaplan \& B. Sadock (Eds.), Comprehensive group psychotherapy (pp. 138-144). Baltimore: Williams \& Wilkins.

Budman, S.H., Bennett, M.J., \& Wismeski, M.J. (1981). An adult developmental model of short-term group psychotherapy. In S.H. Budman (Ed.) Forms of Brief Therapy (pp. 305-342). New York: The Guilford Press. 
Budman, S.H., Clifford, M., Bader, L., \& Bader, B. (1981). Experiential pre-group preparation and screening. Group, 5, 19-26.

Budman, S.M., Demby, A., Feldstein, M. \& Gold, M. (1984). The effects of time-limited group psychotherapy: A controlled study. Intermational Journal of Group Psychotherapy, 34, 587-603.

Budman, S.M., Demby, A., \& Randall, M. (1980). Short-term group psychotherapy: Who succeeds, who fails? Group, 4, 3-16.

Budman, S.H., \& Gurman, A.S. (1983). The practice of brief therapy. Professional Psychology, 14, 277-292.

Budman, S.H., Randall, M., \& Demby, A. (1981). Outcame in short-term group psychotherapy. Group, 5, 37-51.

Campbell, D.T., \& Stanley, J.C. (1963). Experimental and quasiexperimental designs for research. Chicago: Rand McNall.

Charach, R. (1983). Brief interpretive group psychotherapy with early latency-age children. International Journal of Group Psychotherapy, $33,349-364$.

Cohen, J. (1960). A coefficient of agreement for nominal scales. Educational and Psycholocical Measurement, 20, 37-46.

Cohen, J. (1977). Power analysis for the social and behavioral sciences. New York: Academic Press.

Connelly, J.I. (1985). An analysis of therapeutic work behavior for selection and composition in group psychotherapy. (Doctoral Dissertation) Department of Psychology, MoGill University, Montreal.

Connelly, J.I., \& Piper, W.E. (In press). An analysis of pretraining work behavior as a composition variable in group psychotherapy. International Journal of Group Psychotherapy.

Connelly, J.I., Piper, W.E., \& Rraha, R. (1.985). The therapeutic work rating system (TWS) manual. Umpublished document.

Conneliy, J.I., Piper, W.E., de Carufel, F.I., \& Debbane, E.G. (1986). Premature terminations in group psychotherapy: Pretherapy and early therapy predictors. International Journal of Group Psychotherapy, 36, 145-152.

D'Augelli, A.R. (1973). Group composition using interpersonal skills: An analogue study on the effects of members' interpersonal skills on peer ratings and group cohesiveness. Journal of counselling Psychology, 20, 531-534.

Davanloo, H. (1978). Principles and techniques of short-term dymamic psychotherapy. New York: Spectrum Press. 
Debbane, E.G., de Carufel, F.L., Bienvemu, J.P., \& Piper, W.E. (1986). Structures in interpretations: A group psychoanalytic perspective. International Joumal of Group Psychotherapy, 36.

Derogatis, L.R. (1977). SCL-90 administration, scoring and procedures mamual-I. Baltimore: John Hopkins University Press.

Derogatis, L.R., \& Cleary, P.A. (1977). Confirmation of the dimensional structure of the SCI-90: A study in contrast validation. Joumal of Clinical Psychology, 33,981-989.

Derogatis, I.R., Rickels, K., \& Rock, A.F. (1976). The SCI-90 and the MMPI: A step in the validation of a new self-report scale. British Joumal of Psychiatry, 128, 280-289.

Dies, R.R. (1979). Group psychotherapy: Reflections on three decades of research. Joumal of Applied Behavioral Science, 15, 361-374.

Dies, R.R. (1983a). Bridging the gap between research and practice in group psychotherapy. In R.R. Dies \& K.R. Mackenzie (Eds.), Advances in group psychotherapy Integrating research and practice (pp. 1-26). New York: International Universities Press.

Dies, R.R. (1983b). Clinical implications of research on leadership in short-term group psychotherapy. In R.R. Dies \& K.R. Meckenzie (Eds.), Advanoes in group psychotherapy Integrating research and practice (pp. 27-28). New York: International Universities Press.

Dies, R.R. (1985). Leadership in short-term group therapy: Manipulation or facilitation? International Journal of Group Psychotherapy, 35, 435-455.

Dixon, W.J. (Ed.). (1983). BMDP Statistical software 1983 printing with additions. Berkeley: University of California Press.

Dinning, W.D., \& Evans, R.G. (1977). Discriminant and convergent validity of the SCL-90 in Psychiatric Impatients. Joumal of Personality Assessment, 41, 304-309.

Drob, S., \& Bernard, H.S. (1985). Tho models of brief group psychotherapy for herpes sufferers. Group, 2, 14-20.

Drob, S., \& Bernard, H.S. (1986). Time-limited group treatment of genital herpes ratients. International Journal of Group Psychotherapy, 36, 133-144.

Elbirlik, K. (1983). The mourning process in group therapy. International Journal of Group Psychotherapy, 33, 215-227.

Erikson, E.H. (1950) Childhood and society. New York: Norton. 
Ferenczi, S., \& Rauk, O. (1925). The development of psychoanalysis. New York: Nervous and Mental Disease Publication Company.

Ferguson, G.A. (1981). Statistical analysis in psychology and education (5th ed.). U.S.: MoGraw Hill Inc.

Fisher, R.A., \& Yates, F. (1963). Statistical tables for biological, agricultural, and medical research (6th ed.). New York: Hafner Publishing Co., Inc.

Frank, J.D. (1975). Group psychotherapy research 25 years later. International Journal of Group Psychotherapy, 25, 159-162.

Freud, S. (1914). On narcissism: An introduction. In J. Stachey (Ed. and Trans.) The standard edition of the complete psychological works of Sigmund Freud (Vol. 14, pp. 69-102). Iondon: Hogarth Press, 1957.

Freud, S. (1917). Mourning and melancholia. In J. Stachey (Ed. and Trans.) The standard edition of the complete psychological works of Sigmund Freud (Vol. 14, pp. 243-258). Iondon: Hogarth Press, 1963.

Freebury, R. (1986). Psychoanalytic perspectives on object loss: Past and present. Unpublished paper.

Furst, W. (1975). Homogeneous versus heterogeneous groups. In $M$. Rosenbaum, \& M.M. Berger (Eds.), Group psychotherapy and group finction (rev. ed.) (pp. 409-412). New York: Basic Books.

Golin, S., \& Hartz, M.A. (1979). A factor analysis of the Beck depression inventory in a milaly depressed population. Journal of Clinical Psychology, 33, 322-325.

Goldberg, D.A., Shuryler, W.R., Bransfield, D. \& Savino, P. (1983). Focal group psychotherapy: A cymamic approach. International Journal of Group Psychotherapy, 33, 413-431.

Goldstein, A.P., Heller, K., \& Sechrest, L.B. (1966). Psychotherapy and the psychology of behavior change. New York: John Wiley.

Gough, H.G. (1956). Califormia Psychological Inventory. Palo Alto, Califomia: Consulting Psychological Press.

Green, B.I., Gleser, G.C., Stone. W.N., \& Seifert, R.F. (1975). Relationships among diverse measures of psychotherapy outcome. Joumal of Consulting and Clinical Psychology, 43, 689-699.

Grunebaum, H., \& Kates, W. (1977). Whom to refer for group psychotherapy. American Journal of Psychiatry, 134, 130-133.

Gurland, B.J., Yorkston, M.J., Frank, J.D., \& Fliess, J.I. (1972). The structured and scaled interview to assess maladjustment (SSIAM): I. Description, rationale and development. Archives of General 
Psychiatry, 27, 259-263.

Hayes, S.I., \& Haas, J.R. (1988). A Reevaluation of the concept of clinical significance: coals, methods, and methodology. Behavioral Assessment, 10, 189-196.

Herman, J., \& Schatzow, E. (1984). Time-limited group therapy for women with a history of incest. International Joumal of Group Psychotherapy, 34, 605-616.

Hill, W.F. (1965). Hill Interaction Matrix. Los Angeles: University of Southem California Youth Study Center.

Hirshfeld, R.M.A., Kleman, G.I., Gough, H.G., Barrett, J., Korchin, S.J., \& Chodoff, P. (1977). A measure of interpersonal dependency. Joumal of Personality Assessment, 4I, 610-618.

Hollon, S.D., \& Flick, S.N. (1988). On the meaning and methods of clinical significance. Behavioral Assessment, 10, 197-206.

Horowitz, M., Wilner, N., \& Alvarez, W., (1979). Impact of event scale: A measure of subjective stress. Psychosomatic Medicine, 4I, 209-218.

Horowitz, M.J., Krupnick, J., Kaltreider, N., Wilner, N., Ieong, M.S., \& Marmar, C. (1981). Initial psychological response to parental death. Archives of General Psychiatry, 38, 316-323.

Horowitz, M.J., Marmar, C., Weiss, D.S., DeWitt, K.N., \& Rosenbaum, R. (1984). Brief psychotherapy of bereavement reactions. Archives of General Psychiatry, 41, 438-448.

Horowitz, M.J., Wilner, N., Marmar, C., \& Krupnick, J. (1980). Pathological grief and the activation of latent self-images. The American Joumal of Psychiatry, 137, 1157-1162.

Horwitz, L. (1.976). Indications and contraindications for group psychotherapy. Bulletin of the Menringer Clinic, 40, 505-507.

Hudson, W.W., Thyer, B.A., \& Stocks, J.T. (1985). Assessing the importance of experimental cutcomes. Joumal of Social Service Research, 8, 87-98.

Imber, S.D., Lewis, P.M., \& Loiselle, R.H. (1979). Uses and abuses of the brief intervention group. International Joumal of Group Psychotherapy, 35, 309-330.

Jacobson, N.S., \& Revenstorf, D. (1988). Statistics for assessing the clinical significance of psychotherapy techniques: Issues, problems, and new developments. Behavioral Assessment, 10, 133-145.

Jackson, D.M. (1974) - Personality Research Form Mamual. Goshen, N.Y.: 
Research Psychologist's Press.

Kaul, T.J., \& Bednar, R.In (1978). Conceptualizing group research A preliminary analysis. Small Group Behavior, 9, 173-191.

Kaul, T.J., \& Bednar, R.I. (1986) Experiential gram research: Results, questions and suggestions. In S.I. Garfield and A.E. Bergin (Eds.), Handbook for psychotherapy and behavior change, (3rd ed.) (pp. 671-714). New York: John Wiley \& Sons, Inc.

Kendal1, P.C., \& Grove, W.M. (1988). Normative comparisons in therapy outcome. Behavioral Assessment, 10, $147 \cdot 158$.

Kernaleguen, A. \& Conrad, G. (1980). Analysis of five measures of self-concept. Perceptual and Motor Skil1s, 51, 855-861.

Kernberg, O.F. (1973). Sumnary and conclusions of "Psychotherapy and psychoanalysis, final report of the Menuinger Foundation's psychotherapy research project." Intemational Joumal of Psychiatry, 11, 62-77.

Kernberg, O., Burstein, E., Coyne, I., Appelbaum, H., Horwitz, I., \& Voth, H. (1972). Psychotherapy and psychoanalysis: Final report of the Menninger Foundation's psychotherapy research project. Bulletin of the Menninger Clinic, 36, 1-275.

Kein, M. (1948). Contributions to psychoanalysis 1921-1945. Iondon: Hogarth.

Klein, R.H. (1983). Some problems of patient referral for outpatient group psychotherapy. International Journal of Group Psychotherapv, $33,229-241$.

Klein, R.H. (1985) . Some principles of short-term group therapy. International Journal of Group Psychotherapy, 35, 309-330.

Klein, R.H., \& Carroll, R.A. (1986). Patient characteristics and attendance patterns in outpatient group psychotherapy. International Journal of Group Psychotherapy, 36, 115-131.

Lambert, M.J., christensen, E.R., \& DaJulio, S.S. (1983). The assessment of psychotherapy outcome. New York: John Wiley \& Sons.

Lambert, M.J., Shapiro, D.A., \& Bergin, A.E. (1986). The effectiveness of psychotherapy. In S.I. Garfield and A.E. Bergin (Eds.), Handbooks for psychotherapy and behavior change, (3xd ed.) (pp. 157-212). New York: John Wiley \& Sons, Inc.

Lapointe, K.A., \& Rimm, D.C. (1980). Cognitive, assertive, and insight-oriented group therapies in treatment of reactive depression in women. Psychotherapy: Theory, Research, and Practice, 17, $312-321$. 
Ierner, H.D., \& Lemer, P.M. (1987). Separation, depression, and object loss: Implications for nancissism and object relations. In $\mathrm{J}$. Bloom-Feshback, S. Bloom-Feshback, and Associates (Eds.), The psychology of separation and loss (pp. 375-395). San Francisco: Jossey-Bass Publishers.

Iieberman, M.A., \& Videka-Sherman, I. (1986). The impact of self-help groups on the mental health of widows and widowers. American Journal of Orthopsychiatry, 56, 435-449.

Iocke, N.M. (1961). Group Psychoanalysis. New York: University Press.

Iothstein, L.M. (1978). The group psychotherapy dropout phenomenon revisited. American Joumal of Psychiatry, 135, 1492-1495.

Iuborsky, I. (1984). Principles of psychoanalytic psychotherapy. New York: Basic Books.

Iuborsky, L., Mintz, J., Auerbach, A., Christoph, P., Bachrach, H., Todd, T., Johnson, M. , Cohen, M. , \& O'Brien, C.P. (1980). Predicting the outcome of psychotherapy. Archives of General Psychiatry, 37, 471-481.

Mackenzie, K.R., \& Livesley, W.J. (1983). A developmental model of brief group therapy. In R.R. Dies \& K.R. Mackenzie (Eds.), Advances in group psychotherapy Integrating research and practice (pp. 101-116). New York: International Universities Press.

Malan, D.H. (1976a). The frontier of brief psychotherapy. New York: Plemm Press.

Malan, D.H. (19766). Toward the Validation of Dynamic Psychotherapy. New York: Plenum Medical Book Company.

Mann, J. (1973). Time-limited psychotherapy. Cambridge, MA: Harvard University Press.

Marmar, C.R., Horowitz, M.J., Weiss, D.S., Wilner, N.R., \& Kaltreider, N.B. (1988). A controlled trial of brief psychotherapy and mutual-help group treatment of conjugal bereavement. American Journal of Psychiatry, 145, 203-209.

Mathiasen, E.H., \& Daverport, Y.B. (1988). Reciprocal depression in recovering alcoholic corples: The efficacy of the psychodynamic group treatment. Group, 12, 45-55.

Mccallum, M., \& Piper, W.E. (1987). Psychological Mindedness Assessment Procedure (PMPP). Umpublished manual.

Mccallum, M., \& Piper, W.E. (1988). Psychoanalytically oriented short-term groups for outpatients: Unsettled issues. Group, 12, 21-32. 
MoNair, D.M. (1976). Discussion: comments on the Menninger Project. In R.I. Spitzer and D.R. Klein (Eds.), Evaluation of psychological therapies, psychotherapies, behavior therapies, drug therapies and their interactions (pp. 57-61). Baltimore, Maryland: The John Hopkins University Press.

Melnick, J., \& Woods, M. (1976). Analysis of group composition research and theory for psychotherapeutic and growth-oriented groups. Journal of Applied Behavioral Science, 12, 493-512.

Miller, R.G. (1981). Simultaneous Statistical Inference (2nd ed.). New York: Springer-Verlag.

Mintz, J., Luborsky, L., \& Christoph, P. (1979). Neasuring the outcomes of psychotherapy: Findings of the Penn psychotherapy project. Joumal of Consulting and Clinical Psychology, 47, 319-334.

Mullan, H., \& Rosenbaum, M. (1975). In M. Rosenbaum, \& M.M. Berger (Eds.), Group psychotherapy and group function (rev. ed.) (pp. 415-426). New York: Basic Books.

Neumann, M., \& Gaoni, B. (1974). Types of patients especially suitable for analytically oriented group psychotherapy some clinical examples. Israel Annals of Psychiatry and Related Disciplines, 12, 203-215.

Nietzel, M.T., \& Trull, T.J. (1988). Meta-analytic approaches to social comparisons: A method of measuring clinical significance. Behavioral Assessment, 10, 159-169.

Ohlmeier, D., Karstens, R., \& Kohle, K. (1973). Psycho-analytic group interview and short-term group psychotherapy with post-myocardial infarction patients. Psychiar. Clin., $\underline{6}, 240-249$.

Parloff, M.B., \& Dies, R.R. (1977). Group psychotherapy outcome research 1966-1975. International Journal of Group Psychotherapy, 27, $281-319$.

Piper, W.E., Deboane, E.G., Biervenu, J.P., \& Garant, J. (1982). A study of group pretraining for group psychotherapy. International Journal of Group Psychotherapy, 32, 309-325.

Piper, W.E., Debbane, E.G., Biervenu, J.P., \& Garant, J. (1984). A comparative study of four forms of psychotherapy. Joumal of Consulting and Clinical Psychology, 52, 268-279.

Piper, W.E., Debbane, E.G., Bienvem, J.P., \& Garant, J. (1986). Relationships between the focus of therapist interpretations and outcome in short-term, individual psychotherapy. British Journal of Medical Psychology, 59, 1-11.

Piper, W.E., Debbane, E.G., de Carufel, F.I., \& Bienvenu, J.P. (1987). 
A system for differentiating therapist interventions from other interventions. Bullecin of the Menninger Clinic, 51, 532-550.

Piper, W.E., Debbane, E.G., \& Garant, J. (1977a). Group therapy cutcome research: problems and prospect's of a first-year project. International Journal of Group Psychotherapy, 27, 321-341.

Piper, W.E., Debbane, E.G., \& Garant, J. (1977b). An outcome study of group therapy. Arahives of General Psychiatry, 34, 1027-1032.

Piper, W.E., Debbane, E.G., Garant, J., \& Bienvemu, J.P. (1979). Pretraining for group psychotherapy: A cognitive-experiential approach. Archives of General Psychiatry, 36, 1250-1256.

Piper, W.E., Doan, B.D., Echwards, E.M., \& Jones, B.D. (1979) . Cotherapy behavior, group therapy process and treatment outcome. Journal of Consulting and Clinical Psychology, 47, 1081-1089.

Piper, W.E., Jones, B.D., Lacroix, R., Marrache, J., \& Richardisen, A.M. (1984). Pregroup interactions and bonding in small groups. Small Group Behavior, 15, 51-62.

Piper, W.E., \& Ieonoff, D.J. (1983). Patient distortion in reporting outcome: A procedure for correcting patient outcome scores. Group, 2. $33-42$.

Piper, W.E., \& Marrache, M. (1981). Selecting suitable patients: Pretraining for group therapy as a method of patient selection. Small Gioup Behavior, 12, 459-475.

Pifuer, W.E., Marrache, M., \& Koenig, A. (1986). Relationships between eurly and final reports of treatment outcome in long-term group psy whotherapy. Group, 10, 85-93.

Piper, W.E., Marrache, M., Lacroix, R., Richardsen, A.M., and Jones, B.D. (1983). Cohesion as a basic bond in groups. Hhman Relations, 36, 93-108.

Piper, W.E., \& Mocallum, M. (1988). Psychodynamic Work and Object Rating System (FWORS). Umpublished mamual.

Piper, W.E., Montvila, R.M., \& MoGihon, A.I. (1979). Process analysis in therapy grouns: A behavioral sampling technique with many potential uses. In D. Upper and S.M. Ross (Eds.), Behavioral group therapy: An ammal review, Champaign, Illinois, Research Press.

Poey, K. (1985). Guidelines for the practice of brief, dymamic group therapy. International Joumal of Group Psychotherapy, 35, 331-354.

Reynolds, W.M., \& Gould, J.W. (1981). A psychometric investigation of the standard and short form Beck Depression Inventory. Journal of Consulting and Clinical Psychology, 49, 306-307. 
Richardsen, A.M., \& Piper, W.E. (1986). Leader style, leader consistency, and participant personality effects on learning in small groups. Human Relations, 39, 817-836.

Rosenberg, M. (1979). Conceiving the self. New York: Basic Books.

Rubin, S.S. (1984). Mourning distinct from melancholia: The resolution of bereavement. British Journal of Medical Psychology, 57, 339-345.

Russakoff, I.M., \& Oldham, J.M. (1984). Group psychotherapy on a short-term treatment unit: An application of object relations theory. International Journal of Group Psychotherapy, 34, 339-353.

Saunders, S.M., Howard, K.I., \& Newman, F.I. (1988). Evaluating the clinical significance of treatment effects: Norms and rormality. Behavioral Assessment, 10, 207-218.

SAS Institute. (1985). SAS User's guide: Statistics 1985 edition. Cary, North Carolina: Author.

Sadock, B.J. (1983). preparation, selection of patients, and organization of the group. In H. Kaplan \& B. Sadock (Eds.), Comprehensive group psychotherapy (pp. 23-32). Baltimore: Williams \& wilkins.

Sethna, E.R. \& Harrington, J.A. (1971). Evaluation of group psychotherapy. British Jourmal of Psychiatry, 118, 641-658.

Sifneos, P.E. (1979). Short-term psychotherapy: Evaluation and technique. New York: Plemm Press.

Sifneos, P.E. (1981) Short-term anxiety-provoking psychotherapy: Its history, techniseu, outcomeand instruction. In S.H. Bucman (Ed.), Forms of brief therapy (pp. 45-81). New York: Guilford Press.

Sloane, R.B., Staples, F.I., Cristol, A.H., Yorkston, N.J., \& Whipple, K. (1975). Psychotherapy versus behavior therapy. Cambridge, Massachusetts: Harvard University Press.

Small, I. (1979) - The briefer psychotherapies. New York: Bruner/Mazel Inc.

SPSS Incorporated. (1988). SPSS user's guide (3rd ed.). Chicago: Author.

Smith, M.I., Glass, G.V., \& Miller, T.I. (1980). The benefits of psychotherapy. Baltimore. Maryland: The Johns Hopkins University Press.

Stava, L.J., \& Bednar, R.I. (1979). Process and outcome in encounter groups The effects of group composition. Small Group Behavior, 10, 200-213. 
Stiles, w.B. (1988). Psychotherapy process-autcome correlations may be misleading. Psychotherapy, 25, 27-35.

Stone, W.N., \& Rurtan, J.S. (1984). Duration of treatment in group psychotherapy. International Journal of Group Psychotherapy, 34, 93-109.

Strupp, H.H., \& Binder, J.L. (1984). Psychotherapy in a new key. New York: Basic Books.

Strupp, H.H., \& Hadley, S. (1977). A tripartite model of mental health and therapeutic outcomes. American Psychologist, 32, 187-196.

Stuart, M.R., \& Mackey, K.J. (1977). Defining the differences between crisis intervention and short-term therapy. Hospital and community Psychiatry, 28, 527-529.

Tahka, V. (1984). Dealing with object loss. Scandinavian Psychoanalytic Review, 7, 13-33.

Tolor, A., \& Reznikoff, M. (1960). A new approach to insight: A preliminary report. Journal of Nervous and Mental Disease, 130, 286-296.

Toseland, R.W., \& Siporin, M. (1986). When to recommend group treatment: A review of the clinical and research literature. Intermational Journal of Group Psychothyrapy, 36, 171-201.

Tyson, R.L., \& Sandler, J. (1971). problems in the selection of patients for psychoanalysis: commerts on the application of the concepts of "indications", "suicability" and "analysability". British Journal of Medical Psychology, 44, 211-228.

Tyson, R.I. (1983). Some narrissistic consequences of object loss: a developmental view. Psychoanal tic Quarterly, III, 205-224.

Vachon, M.L.S., Iyall, W.A.L., Rogers, J., Freedman-Ietoesky, \& Freeman, S.J.J. (1980). A contrilled study of self-help intervention for widows. American Journal of Psychiatry, 137, 1380-1384

Waskow, I.E., \& Parloff, M.B. (1975). Psychotherapy Change Measures. Washington, D.C.: LHEW

Weissman, M.M., Paykel, E.S., \& Siegel, R. (1972). Social adjustment scale: Rationale, reliability, validity and scoring. Umpublished document.

Winston, A. (Ed.). (1985). Clinical and research issues in short-term dynamic psychotherapy. Washington, D.C.: American Psychiatric Press, Inc.

Woods, M., \& Melnick, J. (1979). A review of group therapy selection 
criteria. Small Groum Behavior, 10, 155-175.

Yalom, I.D. (1985). The theory and practice of group psychotherapy (3rd ed.), New York: Basic Books.

Yalom, I.D., \& Vinograciov, S. (1988). Bereavement groups: Techniques and themes. International Journal of Group Psychotherapy, 38, 419-457.

Zilberg, N.J., Weiss, D.S., \& Horowitz, M.J. (1982). Impact of event scale: A cross-validation study and some empirical evidence supporting a conceptual model of stress response syndromes. Journal of Consulting and Clinical Psychology, 50, 407-414. 


\title{
APPENDIX A. Informing Referral Sources of the STG Program
}

\author{
Short-term Group Therapy for Lass
}

The External Psychiatric Services of the University of Alberta Hospitals will be conducting short-term grcup therapy for a specialized patient population. Suitable candidates for these groups are men and women, between twenty and sixty years old, who have lost either a spouse, partner, parent, family member or friend as the result of a separation, divorce, death or geographical move. It should have been at least three months since the loss occurred. Hence, although the patient will no longer be experiencing the immodiate effects of the crisis, it should be clear to the assessor that tire patient is still affected by the loss as reflected in his/her inability to resume a happy or satisfying way of life.

Each therapy group will be composed of eight members and therapy sessions will be offered once a week for twelve weeks, with each session lasting one and a half hours. Members will be expected to attend all sessions. The focus of therapy will be psychodynamic rather than supportive such that members will be encouraged to explore their conflicts with respect to the loss of the significant relationship and the (unconscious) reasons behind its contimued interference with their lives. Each group will be led by one of the EPS therapists who have extensive experience with conducting group therapy utilizing the psychodynamic approach.

As part of the EPS's commitment to the evaluation and contimual development of its services, these therapy groups will be monitered by the Research and Evaluation Unit of the clinic. This evaluation procedure includes the assessment of patients utilizing questionnaires and individual interviews in addition to audiotaping the sessions. Hence, patients should be informed of these evaluation procedures and our commitment to the protection of confidentiality. 
APPENDIX B. Therapist Mamual

Short-Term (Time-Iimited) Psychodymamic Group Psychotherapy: Technical Considerations.

\section{1) The Psychodynamic Approach}

Utilization of the psychodynamic approach to STG therapy is based on the assumption that patients' presenting complaint of their inabi?ity to resolve a particular, current problem or crisis, reflects the re-emengence or continued influence of unresolved intrapsychic conflicts. In other words, their reaction to the precipitating stressor (for which they are seeking therapy) is linked to unresolved, unconscious conflicts.

For example, adults who have recently experienced the loss of a significant person may present for therapy complaining of a variety cif disturbances. These disturbances may relate to symptoms characteristic of Affective Disorders (e.g. Major Depresssion; Dysthymic Disorder) or Anxiety Disonders (e.g. Agoraph.obia; Generalized Anxiety Disorder). As varied as the presenting complaints may be, by taking a complete case history, cormon themes may emerge. These themes possibly relate to: wanting yet fearing a close relationship with another person; wanting to be autonomous or self-sufficient yet fearing isolation or loneliness; confusing, conflictual feelings of anger, guilt, depression, in response to the loss of the other (either by separation, divorce, geographical move or death).

\section{2) The Therapeutic Goal}

The therapeutic goal in psychodynamic therapy, therefore, is to promote insight intu the unoonscious conflicts which are inhibiting the patient's ability to resolve the current crisis or problem and is not solely to alleviate the ego-dystonic symptoms. With this patient population, therefore, the therapeutic goal would not be solely to alleviate the presenting complaint of depression or anxiety but to clarify and interpret how these presenting complaints are manifestations of the underlying conflicts referred to above.

\section{3) The Short-Term Modality}

Short-term therapy, by its very nature, cannot explore the entire range of conflicts existing in patients seeking therapy. Nevertheless, advocates of short-term therapy are confident that a thorough exploration and understanding of a particular conflict will benefit patients by offering them an important model for understanding subsequent and/or concurment problems and conflicts in their lives. In addition, the short-term modality has been shown to accelerate the pace of therapy and to yield successful therapy outcomes. Short-term therapy in this project refers to therapy offered for 12 weeks with one session per week of one and a half hours duration. 
The time-limited aspect of SIG promotes the emergence of themes associated with all intrapsychic conflicts. The conmon wish of group members that the group contimue past its pre-determined termination date, represents a wish for the unattainable. This wish for the unattainable is reminiscent of the conflictual, unattainable wishes that are denied gratification by the superego. In addition, the frustrating aspects of the group in terms of not being a 'cure-all' is reminiscent of the anger and frustration that all patients feel with respect to the imperfection of themseives and the world. By accepting the limitations and frustrating aspects of STG, members hypothetically develop ego strength which helps them to resolve subsequent/concurrent non-gratifying situations outside the group. Finaliy, for members conflicted by dependency needs, termination represents a unique opportunity for them to explore the related fears of isolation and abandorment.

Offering this type of therapy to patients experiencing a significant person loss would offer them the opportunity to explore their conflicts about being in a relationship. For example, they may gain insight into how their relationship with this lost person contimues to influence their interactions with the opposite sex. Their conflicts with respect to the lost relationship may, to some extent, influence their reaction to any intimate relationship - be it with same sexed Friends or their children. They may then begin to understand their needs, fears and defenses with respect to forming an intimate relationship with anyone in their lives which would help them understand their needs, fears and defenses with respect to the loss.

\section{4) Group Homogeneity}

To enhance the effiracy of the short-term modality in group therapy, the groups are composed of a homogeneous patient population. Homogeneity refers to themes or conflicts shared by all group members. The homogeneity of the members' conflicts or themes acts to focus the group work. Homogeryeity is also assumed to promote cohesiveness among the members as they recognize and identify witl the similar complaints or concerns of the members. This similarity is elsewhere referred to as "universality" - which is assumed to be a curative factor in group psychotherapy (Yalơil).

The homogeneous nature of the crroups in this project relates to choosing adults who have recently suffered a significant person loss as the patient population. This will facilitate the cohesion of the groups as each member identifies with the problems of other members as their problems will be reminiscent of his/her own. Hence, the group themes or conflicts will quickly come to the fore and members will not feel so alone, isolated or hopeless.

\section{5) Implications for Therapist Activity}

a) Given the short-term format, the therapist focuses on the limited and realizable goal of exploring the common themes or conficts 
of the group. Hence, he/she will not pursue an idiosyncratic or unique problem of any one member but will tenaciously stay with only those conflicts common to all group members.

b) Given the insight-oriented approach, the therapist's primary task is to explore the previously unrecognized conflicts that are linked to the central themes or conflicts. He/she will interpret, therefore, the unconscious dynamics of the group. Discussions of symptoms of depression and anxiety are considered a secondary therapeutic task in this type of therapy. Discussions of medications, are not considered a therapeutic task in psychodynamic therapy.

c) Given that therapy is provided in a group format, the therapist focuses on how these unconscious conflicts are emerging (manifested) in the "here and now" behavior in the group. For example, with this patient population, the therapist may interpret the way:

1. members' fears of intimacy are being displayed in the lack of disclosure between the members or statements reflecting rationalizations such as 'why get involved if it's only for 12 weeks';

2. members' ambivalence over dependency needs is being displayed in their anger at other members' neediness, despair of 'the blind ieading the blind' and their disappointment with the therapist;

3. members' wish to be taken care of is being displayed in demands that the therapist give advice, be more active, answer their questions etc.;

4. members' anger over the loss of the relationship is being displayed in their anger over the absenteeism and dropping out of other members and of the inevitable termination of the group;

5. members' guilt over the loss of the relationship is being displayed in the protectivn (defensive) manoeurres towards absent and dropping out members.

d) Given that the therapy is short-term and insight-oriented, the therapist is active in rapidly focusing on the group defenses, transference reactions and the unconscious themes by utilizing the techniques of clarification, confrontation and interpretation. In addition, the therapist is ever-mindful of group behavior reflecting the termination issue and clarifies, confronts and interprets the conflicts associated with it. For example, the therapist would interpret fear of termination if the members began to:

1. devalue the groun as a way of avoiding the sadness (as they may have done with past losses);

2. rocome helpless or in crisis as a way of blackmailing the therapist to lengthen the duration of therapy;

3. 'fly into health' as a way of denying their need for and therefore loss of the group (as they may have done with past losses);

4. talk of dropping out of therapy as a defense against the feelings of helplessness concerning the end of the group 
("I'll reject you before you have a chance to reject me" syndrome).

6) Characteristic Stages of Process in STG*

1. Exposition - (sessions 1 \& 2) - Members introduce themselves and articulate the central themes. The therapist encourages discussion and actively interprets the group's resistance to intimacy and commitment.

2. Dependency - (sessions 2-4) - Members express anger and neediness for murturance and guidance. The therapist actively interprets the conflictual nature of their feelings and how they are disguised - especially with respect to their feelings towards the leader.

3. Conflict Elaboration - (sessions 4-10) - Members increasingly appreciate the conflictual nature of their feelings. The therapist continues to clarify and interpret the common conflicts in addition to transference and termination reactions.

4) Termination - (sessions 10 - 12) - Members experience a return of earlier behavior and fears and alternate between wishing for and fearing intimacy. The therapist recapitulates the major insights gained from the therapy while interpreting manifestations of the conflict between intimacy and alienation.

*Elaboration of process presented in Goldberg, etal., 1983 
APPENDIX C. Guidelines for Referral to STG.

REFERRAL FROCEDURE FOR SHORT-TERT GROUP PSYCHOTHIIRAPY (STG)

The intake therapist performs the initial crucial tasks of assessing and referring patients to the STG project. The following outline serves as a guide for STG referral decisions. Intake therapists are welcome to approach members of the research unit at any time to discuss patients being considered for referral.

\section{Determining patient suitability for STG}

a) Clinical Considerations.

suitable patients will meet the following criteria:

1. Adult man or woman (ages $20-60$ ) who has lost a significant person (spouse, partner, family member, friend) through separation, divorce, death or geographical move.

2. The loss will have occurred at least three months before presentation at the WIC. While the patient is no longer in crisis, the intake therapist views the loss as centrally influencing the patient's inability to enjoy a satisfying and productive life.

3. The patient agrees to a 12 session contract, prearranged group therapy times, and no concument therapy.

4. The patient is seen as capable of exploring the impact of, and feelings associated with the loss in a group situation (with 6 or 7 other people who have experienced a similar loss experience).

Note: We are interested in acopting patients for STG who span most of the range of patient suitability with respect to the capacity to work within a psychodynamic approach. However, a certain minimal level of ability and motivation to work within this approach is required. Hence, the patient would not be soleiy seeking medication or advice.

b) Evaluative considerations.

The patient must be willing to have his/her therapy evaluated. This process includes:

1. The utilization of a cape recorder and one-way mirror facility.

2. Some additional assessment interviews.

3. Some additional questionnaires and ratings.

4. The possibil:ty that the onset of therapy will be delayed for 10-12 weeks.

\section{Presenting the Clinical and Evaluative Aspects of STG.}

We recommend that you present STG first as a form of treatment that is offered in the WIC, and second as a form of treatment that is being 
evaluated. To some degree all treatments in the WIC are under evaluation, i.e. data are reconded throughout the patient's contact and the type of outcome is officially noted as part of the patient's discharge record. As with STI, STG will be evaluated more thoroughiy than some of our other treatments as yet have been. We would prefer that patients view evaluation as a routine aspect of how we work in the WIC rather than an unusual aspect. The disadvantages associated with the present evaluation are evident (extra assessment time, possible delay of therapy). The advantages are just as important to mention. Each patient will receive 12 sessions of a therapy that has been especially tailored to his/her problem. The therapists are experienced full-time members of our staff. Each patient will be followed closely for a period of $9-12$ months. STG is not "experimental" nor a particularly new form of therapy at the WIC. While the disadvantages need not be minimized, the advantages can be highlighted as part of establishing a realistic optimism about working within this form of therapy.

\section{Obtaining Informed Consent.}

A special Information and consent Form has been prepared for the patients. On the first page is the information concerning their therapy. Patients can take this (information) page home with them, together with an outline of the ground rules of the group. By taking the information and ground rules sheets home with them, we have found that patients' anxiety with respect to their expectations about the group therapy diminishes while thieir comitment increases. On the second page is a line for the patient's signature which you will witness and date. This seoond page is submitted to Fyre Bahrey (the group co-ordinator), together with your referral fon (see below).

\section{Informing the Research and Evaluation Unit (REU) of the Referral.}

As with any group referral, the intake therapist completes a referral form. A special SIG referral form has been designed for this project. It will be attached to the Information and consent form and the ground rules sheet with this package being located in the front office. As mentionsd above, the completed referral form and consent form are submitted to Fyfe Bahrey who - after verifying the appropriateness of the patient for STG - forwards the forms to the REU (via research assistant Hilary Morin's mailbox). At this point the intake therapist's treatment responsibilities are recuced to medication follow-ups (if need be) and acministrative tasks such as paperwork.

\section{The REU's Responsibilities.}

The REU contacts the patient upon receipt of the referral and consent forms. An appointment is arranged. This appointment consists of a str. ztured assessment interview and the administration of questionnaires. Patients are told curing this interview whether they will commence group therapy relatively immediately (i.e. as soon as there are enough suitable patients assembled) or after a 10-12 week 
delay. The REU contacts the group therapists and informs them of the patients' phone number. Patients will be reassessed by the REU at the completion of their therapy and six months thereafter to moniter the gains they make and maintain. Waiting list patients are assessed an additional time - at the end of their waiting period. 
APPENDIX D. Patient Ground Rulles for STG.

Short-term Group Therapy

Psychiatric Walk-in Clinic

We believe that cooperation with the following ground rules will enhance your experience and others' experience in group therapy.

\section{Commitment}

I understand that my commitment to the group is for all 12 sessions. The group will meet for ninety minutes each week. Group attendance must be a high priority and unless there is a very good reason, e.g., severe iliness, I will be there each week. In the event of such an absence, I will notify the therapist prior to the groum and at the next meeting I will share my reason with the rest of the group. I also recognize the importance of being on time since lateness interferes with the work of the group. If I am thinking about leaving the group I will let others know and should I decide to leave the group, I will come for one last session where people can say goodbye.

\section{Responsibilities in the Group}

I agree to work toward learning more about my own and others' problems. I will try to be open and self-examining. I will be as honest as possible in sharing what I am aware of in the group, - i.e. thoughts, feelings, fantasies - about myself, other group members (including the therapists) and other people in my life. I understand that I cannot come to group under the influence of alcohol or drugs. It is also not pennissible to smoke, drink, or eat in group. I also understand that physical violence will not be tolerated in the group.

\section{Responsibilities outside of the Groum}

Confidentiality is essential so that each member can feel safe enough to share. I agree that I will not repeat anything that is said in the group outside the group, unless it concerns only myself. I will not share information that might identify any menber of the group.

Extra group socializing may prevent the work in the group. I understand that contact with another member (including the therapist) outside of group may sabotage my own treatment and I agree to discuss the details of any chance outside contacts in the group. 
APPENDIX E. Informed Consent Form

Information about the Therapy and Evaluation Procedures: A Controlled Evaluation of Short-term Group Psychotherapy

\begin{abstract}
Providing therapy for patients is a central part of our work in the Walk-in Clinic. However, it is not the only part. We also continuously evaluate the services that we provide. This involves monitoring the various assessment and treatment activities and obtaining the reactions of the participants. This is accomplished through the use of interviews, questionnaires and tape recordings. The information obtained is used to improve our understanding of what techniques work best with what types of people. This is a slow process that usually takes several years before conclusions are formed.
\end{abstract}

Fatients who participate in therapy have regular contact with treatment staff, e.g., your therapist, and infrequent contact with evaluation staff, e.g., a person who conducts interviews. The two types of staff have different jobs and work rather independently. It is the job of your therapist to work with you toward achieving yoals that you have formulated. It is the job of evaluation stis. to monitor your experience and your progress. However, any prot; is $s$ or concerns that you experience curing therapy should be raised witn your therapist as he/she is the person who is in charge of your treatment. Psychotherapy is an emotionally involving experience that at times can be stressful, i.e. anxiety arousing. conoerns about therapy are usually best resolved in therapy.

Treatment staff and evaluation staff comprise a small muber of professionals who work in our clinic. Considerable care is taken to preserve the confidentiality of information about our patients. Information is coded by muber rather than by name. Taperecondings are erased after information has been obtained. Questicnnaires and tapes are never presented to classes of students for demonstration purposes. We wolld also like to use our one-way mirror facility. Observers will be a smal I number of permanent staff of our clinic who are in trainina. All observers are bound by rules of confidentiality. Given the precautions that we take we have never experienced a problem involving a breach of confidentiality.

The program of thexapy and evaluation that we are recommending consists of:

1. An initial set of interviews for assessment and orientation purposes.

2. Group therapy that will oocur once-a-week for a period of 12 weeks. The group will bogin soon after the initial interviews are completed or about 12 weeks later after a second interview. The starting time will be determined by a standard, randamized procedure.

3. A series of questionnaires that inquire about your experience 
in and outside of your group. These usually take 1-2 hours to complete.

4. An interview concucted just after therapy ends and again six months later by a member of the evaluation staff. This usually takes 1 hour.

5. Monitoring of activities by an audio tapereconder.

\section{Consent Form}

I understand the therapy and evaluation procectures and have had the opportunity to clarify them with a staff member of the Walk-in clinic. This includes the possible benefits and discomforts associated with the procecures. I know that I may ask any questions about the procectures as they progress.

I have been assured that persoral information will be kept confidential and that no information will be released or printed that would disclose personal identity without my permission.

I understand that I am free to withdraw from the evaluation procectures at any time and that my therapy would continue.

I have been given a copy of the information and consent forms.

Signature of Patient

Date

Signature of Witness

The person who may be contacted about the evaluation procedures is:

Dr. Willian Piper

Associate Director, Program Evaluation and Research

Division of External Psychiatric Services

University of Alberta Hospitals

Telephone: 432-6501 


\section{APPENDIX F. Referral Form}

Short-term Ioss-Group Referral Form

Patient's Name:

WIC \#:

Address:

WIC Therapist:

Telephone:

Home work

Age:

Consent Obtained:

Date Referred:

Hours conflicting with Group Therapy:

Axis I Diagnosis:

Axis II Diagnosis:

Current Medications:

None WIC Prescription (Indicate Drug(s)) and amount other Non-prescription (Indicate Drug(s)) and amount

Ioss(es) Experienced By Patient and Date Loss(es) occurred:

Expected Goals, Changes, Gains that You Feel the Patient Could Achieve from the Group Experience:

Reservations/Contraindications for Treatment?

Note: Please attach patient consent form to this form. 
APPENDIX G. Procecure for Independent Assessment

PROCEEDEE FOR INDEPENDENT ASSESSMENT

1 Introduce yourself as a member of the Evaluation Unit

2 (For patient \#1) Give patient a copy of the blank target sheet (which begins "By now you ..."). Say you'll return in 15 minutes.

3 Bring the patient to the office for Psychological Mindedness assessment.

4 Review the morning's events:

"I'll tell you what we'll be doing this morning:

First, I'll show you a vidiotape of simulated therapy sessions \& asik you a few questions about them.

Next, my colleague will 1 ask you a series of questions about various aspects of your life. She will also go over your reasons for entering therapy at this time so we have a clear understanding of your goals.

Then, we'll ask you to complete a few questionnaires.

5 PSYCHOLOGICAL MINDEDNESS ASSESSMENT

"I'm going to show you segments of simulated therapy sessions. A female patient will be describing to her male therapist something that happened to her recently. Then the therapist will come on and say what he thinks might be going on.

"The first time through, I want you to relax and afterwards I'II ask you for your general impression of what seems to be troubling the woman. I'll play it again and then you can feel free to stop me if you want to clarify or elaborate on what you said. But the first time I'll ask you for just a general impression".

PIAY SCENERIO \#I \& then ask: "What seems to be troubling this woman" TAPE RECORD patient's response.

then say: "Fine, I'll piay it again and feel free to stop me if you wish to clarify or elaborate on what you think is troubling her".

then say: "Now the therapist is going to come on and say what he thinks might be going on. This may be the same aspect that you picked up on or it may be a different aspect. I want you to listen and then tell me what he is referring to - what did the woman say that would lead him to thirk that; what is he getting at." 
PLAY DYNAMICS \#1. TAPE RECORD RESPONSE.

then say: "He's going to go on and elaborate. Iisten and tell me if you have arything to add."

PLAY DYNAMICS \#2 \& \#3. RECORD RESPONSE.

then say: "The therapist is now going to talk about another aspect that he sees going on. Iisten and tell me what he's driving at; what he means; where is he getting that from."

PLAY TRANSFERENCE \#1,2,3 (same procecture as dymamics)

then say: "I'm going to play a second senario. We'll follow the same procecture. First, just listen to the woman and I'll ask for a general impression of what's troubling her."

PLAY SCENAARTO \#2 - SAME PROCEDURE AS SCENARIO \#1

If patient asks: it is not the same patient; she's been in therapy a couple of weeks; we don't use a real patient for confidentiality reasons.

IF 2nd PATIENT, give copy of blank target sheet - return in 15 mins.

6 TAKE TO OFFICE FOR OUTCOME ASSESSMENT

A) SFI- "I'm going to ask you a series of qiinstions. They're the same questions I ask everyone. Think about the lust month to respond."

B) TARGEIS- "NOW I'll ask you a few questions about your goals for therapy."

C) INFORMATION- "I will be in touch within a few weeks to tell you whether you will be waiting or not.

If you begin therapy relatively immediately - I'll see you at the end (i.e. in 3 months). Or, if delay, I'll see you in 10 weeks right before you begin therapy. I'll also see you at the end of therapy and 6 months thereafter to see how you are maintaining the gains.

7. TAKE TO OFFICE WHERE QUESTIONNAIRES CAN FE COMILETED

"Now I'Il ask you to complete these quest 1onnaires. If you have any questions I'll be back in half-an-hour to see how you're doing."

"When you're finished, you can hand the forms into the front receptionist."

C) INFORMAITON- Tell them either immediate or delay If immsiate: give card with date, time and therapist on it. 
If delay: give approximate date, time and therapist and say you'll see them in 10 weeks for another appointment and that you'll call to confirm this in 6 weeks.

If this is a pre-therapy assessment, i.e., IBI for imnediate, IB2 for delay say:

"I'll be seeing you when you finish therapy to evaluate the gains you make and 6 months after that to see how you are maintaining the gains you made." 
APPENDIX H. Mamual for the Psychological Mindedness Assessment Procedure

PSYCHOLOGICAL MINDELINESS ASSESSMENT PROCEDURE (PMAP)

THE MANUAL

Subjects are asked to explain what is troubling a patient portrayed on a videotape. These explanations are rated according to their similarity to the psychodynamic explanation. This dimension of similarity is callod "Psychological Mindedness." The rationale for operationalizing "Psychological Mindedness" in this way, is that the more the explanation resembles a psychodynamic formulation, the more the subject shares the basic assumptions held by psychodynamic therapists concerning human pathology. Hence, the more psychologically minded the subjects the more amenable these subjects should be to a psychodynamic therapeutic approach. This amenability has implications for therapeutic process and outcome. 


\section{The Nine Levels of Psychological Mindedness}

Ievel I.

Criterion: The subject identifies a specific internal experience of the patient.

Rationale: A basic assumption of psychodynamic theory is that of "Psychic Determinism," whereby all human functioning results from an internal or psychic process. Hence, in explaining the patient's problem, the psychologically minded person begins by appreciating - or focusing on - the internal experience of the patient.

Ievel II.

criterion: The subject recognizes the driving force of an internal experience of the patient.

Rationale: Psychooynamic theory further postulates that problems, complaints or symptons arise when an "inpulse" is perceived as threatering to the mental apparatus. Hence, the basis of the patient's problen is an impulse or drive which is pressing for expression, or is motivating the patient.

Ievel III.

Criterion: The subject identifies a result of a drive such that a causal link is made between an intermal event and its resultant expression.

Rationale: Psychic determinisin states that the internal or psychic process is responsible for human expressions - in the form of behavior, affect or cognitions. Hence, the internal or motivating force is manifested in an extermal expression.

Ievel IV.

Criterion: The subject recognizes that the motivating force in the patient is largely out of her awareness or is unconscious.

Rationale: A second basic assumption of psychodynamic theory is that of the "Unconscious," whereby the majority of the mental apparatus' functioning is not inmediately accessible to the conscious mind. 
Level V.

Criterion: The subject identifies conflictual components of the patient's experience.

Rationale: The impulse or driving force, is threatening, according to theory, because it is contradictory or incompatible with reality - be it external reality or internal reality. Internal reality relates to superego morality needs or beliefs. The conflict can manifest itself as being between cognitions, affects, needs or any combination of the above.

LeVel VI.

Criterion: subject identifies a causal link where the conflict is presented as generating an expression.

Rationale: conflict produces tension or disequilibrium within the mental apparatus which presses for discharge in order to return the system to a state of equilibrium. Hence, conflict motivates the homeostatic mechanism. The homeostatic mechanism can manifest itself in many ways.

Level VII.

criterion: Subject identifies a causal link where tension (fear, anxiety) is presented as motivating an expression.

Rationale: It is the tension which is intolerable to the system and hence, it is the tension created by the conflict that is the ultimate motivator.

Ievel VıII.

Criterion: subject recognizes that the patient is engaging in a defensive manoeurre.

Rationale: The expression has been filtered through a self-protecting or defensive mechanism. These defense mechanisms distort and prevent the true expression of the conflict.

Ievel IX.

Criterion: Subject recognizes that despite the defensive manoeuver, the patient remains disturbed in some way by the conflict.

Rationale: Pathology results when the defense mechanisms cannot effectively rectuce the tension as no ocmpromise has been reached between the conflictual components. 
Part I: The Patient's Acoount

Scenario \#1.

Patient: I. don't... (silence). I don't know where to begin. (silence) I feel so weind lately; kind of at loose ends. (pause) It started last week and just won't go away. (long pause) I didn't know if I should even tell you this. (silence) I went shopping last Wednesday and while I was walking through Eaton's, I saw my husband - my ex-husband, I mean. There he was not ten feet in front of me at the jewlery counter. At first, I just wanted to rush up to him and say 'hi, long time no see - how about lunch or something.' But then I thought, well, he's probably burying a present for his new girlfriend and he'd just feel awkward - or maybe he'd think I was spying on him - or I don't know what. I started to feel really nervous. I felt like I just had to get out of there. I felt like I was back in high school watching this guy I used to have a terrible crush on. It was Iike I couldn't move or speak - I just stood there, watching. I watched for a long tine, daydreaming... Oh, I don't know, maybe of how nice it would be if he were burying a necklace for me. He'd come home and I'd be cooking dinner. He'd come up behind me and put it around my neck. I'd be so surprised and happy and everything would be okay again (she smiles). Sometimes I really wish he'd come back (pause, she looks very ihoughtfull). But then, I know I'm really better off without him. (She looks very sad). Cod he was such a - I get so angry when I thirk of how it used to be - I sometimes wish I had never met him. Oil, I don't know how I feel, I feel so weird. 
Scenario \#2.

Patient: Was that woman who just left another patient of yours ? She was very pretty. (pause) Well, I went out with Richard last night to this cozy little French restaurant. It was so romantic and I was so happy - just being with him makes me happy. Sometimes I think he's exactly what I've been looking for. He's such a great guy - he could have amy woman he wanted. (pause) We were having such a grod time and then (puase) I had to go and ruin everything. You see, the waitress was really charming and attractive, and I thought, (pause) well, I thought he was being a bit txo friendly with her. I started to feel really ugly and dull. I began to wonder what he sees in me. I felt like I was going to start crying. I wanted to just get up and leave - I was so upset. Well I blew up ac hin saying 'hey if you'd rather be with her - fine. I don't care. you're nothing to me. ' I don't know why I got so angry. My ex-husband and I used to have some terrible fights but with him I had good reason - he was a terrible flirt. But Richard's not like that. Now I'm worried that he'll never call me again. Maybe that would be for the best though. Better he break up with me now than later - when I'm even more involved. I don't want to get hurt again. I don't know if I'm even ready for another relationship. Maybe I need more time. Sometimes I don't even know if I like Richard. On, I don't know - I'm so confused. sometimes I wonder if I'm making any progress at all. You're probably wondering that too. 
Part II: The Assessment of Interpretation comprehension

Interpretations focusing on dynamic components

Scenario \#1.

1) Highest level of ambiguity.

Therapist: Seeing your ex-husband excited you, a kind of nervous excitement. You seem to be trying to discount these feelings.

2) Moderate level of ambiguity.

Therapist: seeing your ex-husband has aroused some positive feelings about him, also some nervous feelings. Your response seems to be 'why want what you don't have'.

3) Lowest level of ambiguity.

Therapist: Seeing your ex-husband made you want to be with him again, and that seems to have made you arxious. Telling yourself that you're better off without him, might be your way to feel less anxious.

Scenario \#2.

1) Highest level of ambiguity.

Therapist: It seems as though you have a real interest in Richard, a rather unsettling interest. You seem to be trying to disown these feelings.

2) Moderate level of ambiguity.

Therapist: It sounds as though you really like Richard and that's stirred up some apprehension. Your response seems to be 'he's not interested in me.'

3) Lowest level of ambiguity.

Therapist: It sounds like you really want Richard and that has aroused your fear of getting hurt. Claiming that he's really not interested in you sounds like a way of protecting yourself. 
Assessment of Interpretation comprehension

\section{Interpretations focusing on the transference}

Scenario \#I.

1) Highest level of ambiguity:

Therapist: You seemed to have some difficulty, today, deciding whether to tell me what happened. I guess the incident in the store was a difficult one for you.

2) Moderate level of ambiguity.

Therapist: Your difficulty and hesitation with me makes me think of the difficulty you had deciding what to do when you saw your ex-husband.

3) Lowest level of ambiguity.

Therapist: You seem to have difficulty approaching men. This showed up in the store with your ex-husband but also here, with me, in your difficulty in telling me what happened.

Scenario \#2.

1) Highest level of ambiguity.

Therapist: You seem to be wondering what I see in you. I gress the incident in the restaurant has left you wondering about your worth.

2) Moderate level of ambiguity.

Therapist: Your doubt about being a good patient reminds me of your doubt about being good enough for Richard.

3) Lowest level of ambiguity.

Therapist: You seem to anticipate rejection. This showed up in the restaurant with Richard, but also here, with me, when you wondered if I think you're not making any progress. 
Criteria for Rating the Level of Psychological Mindedness

Ievel I

The subject identifies an internal experience of the patient but does not integrate this experience or causally relate it to other aspects of the patient.

eg. She's lonely.

She's insecure.

Ievel II

The driving force of the internal experience is explicitly stated. If patient identifies the wish of the patient, this automatically is rated as category II due to the theoretical bacikground of the Wish.

eg. Her whole problem is that she's insecure. It's all due to her loneliness.

The Wish - a desire; need; want.

Scenario \#1 to be with her husband again.

Scenario \#2 to be with Richard or to have a relationship

with Richard.

Ievel III

Causal statements which are stated without appreciation or recognition of the conflictual aspect of the antecedent are rated as category III.

a) Antecedent:

Scenario \#1:

thinking husband will feel awkward thiriking husband will think she is spying

thinking husband is burying a present for his girlfriend.

Effect: she feel nervous

she begins to daydream

she watcines him

she has to 'get out of there' (panics).

b) Antecedent: feeling nervous

Effect: she daydreams

she panics

she watches him.

c) Antecedent: daydreaming about husband coming back

Effect:

feels happy, everything will be ok again. 
d) Antecedent: thinking about the past Effect: feels very sad.

e) Antecedent: thinking about the past fondly Effect: anger.

Scenario \#2:

a) Antecedent: being with Richard Effect: she feels happy she thinks he's a great gry she thirks he's what she has been looking for.

b) Antecedent: thinking waitress is attractive Effect: thinks Richard wants to be with her

c) Antecedent:

thinking Richard is flirting Effect: she feels ugly and durl she feels like crying she wants to leave she wonders what he sees in her

d) Antecedent: feeling upset Effect: anger

says she doesn't care

e) Antecedent: getting angry Effect: anticipates rejection thinks he won't call.

f) Antecedent: not wanting to get hurt again Effect: doesn't know if ready for another relationship doubts herself doesn't know if she likes Richard

Ievel IV.

The subject recognizes that the patient is unaware of certain feelings or that they are largely unconscious.

Scenario \#1: She still thinks of him as her husband even though she doesn't really realize this. 
Scenario \#2: She is not aware of how true it is when she says 'I had to ruin everything.'

\section{Ievel V}

The subject identifies a conflictual wish or an intrapsychic conflict. Key works which reflect that the subject is aware of the conflict are: on the one hand she feels this but on the other hand.... -or - she feels like this but she also feels like that...- or - she can't reconcile herself to the fact... - or - she's ambivalent.

Scenario \#1: She wants to be with her husband but she's also very angry with him: kut she knows she can't have him.

Scenario \#2: She wants a relationship with Richard but she doesn't know if she really likes him; if she's really ready for another relationship.

Note: The latter part of the conflict may actually be a fear or a defense, but the subject is not accentuating this part yet. If the subject goes on to explain how the latter part of the conflict is a fear or a defense, both the conflict and the appropriate cynamic are rated.

Ievel VI

After the conflict is identified, the subjects link one or both aspects of the conflict to a resultant expression. If the resultant expression of the conflict is fear or 'anxiety' then both anxiety and conflict causal are rated.

Scenario \#1: She wants to be with her husband but knows it's over so she fantiasizes about it instead.

Scenario \#2: She wants Richard kut is so insecure that she gets jealous of the waitress.

\section{Level VII}

The subject identifies fear or anxiety in relationship to the wish or conflict.

Note: If fear is stated without recognition or appreciation of its inhibitory or conflictual aspect (i.e. against the wish) then it is rated as a negative affect(category I or II).

Scenario \#1: $\quad$ started to feel very nervous; had to get out of there - after wanting to rush up to him. 
Scenario \#2:

she wants him but she's afraid she's not good enough; she's afraid she'll get hurt; she's afraid he'll break up with her.

\section{Ievel VIII}

The subject identifies the patient's avoidance or distortion of reality; action or thoughts which are contrary to a previously expressed wish or feeling.

Note: The distortirig aspect or unbelievable aspect of the behavior must be identified and stated to be rated as defense.

Scenario \#1: a) denial - fantasy of musband giving her the necklace

b) avoidance - does not approach husband (when she wanted to rush up to him).

c) projection - that husband would feel awkward

d) rationalization - "sour grapes" - 'knows' that she 'really' is better off without the husband (even though she gets sad when she realizes how mach she wants hirn to return).

e) reaction formation - feels angry when she initially felt very happy thinking about him.

Scenario \#2: a) rationalization - it would be best if he never called; maybe she needs more time

b) reaction formation - anger when she feels hurt (felt like crying) saying she doesn't care

c) denial - sometimes she doesn't krow if she even likes Richard

d) projection - she feel that she's not good enough for Richard so thinks Richard must think so too, so believes that Richard would rather be with the waitress.

\section{Level IX}

In addition to identifying the defense, the subject also identifies that which the defense is protecting her against (i.e. the wish, the fear). This integration of the defense with the conflictual aspects of the patient must be explicitly stated. 
Criteria for Rating the Ievel of Interpretation Comprehension

Dymamics

Scenario \#1.

Dynamic:

The wish.

Therapist's statement: 1) Seeing your ex-husband excited you ...

2) seeing your ex-husband aroused some positive feelings ...

3) Seeing your ex-husband made you want to be

Referent: "At first, I just wanted to rush up to him ... with him again ... sometimes I really wish he'd come back."

Dynamic:

Anxiety.

Therapist's statement: 1)... a kind of nervous excitement ...

2)... also some nervous feeling...

3)... and that seems to have made you anxious

Referent:

"I started to feel really nervous. I felt like I just had to get out of there."

Dynamic:

Defense - Rationalization.

Therapist's Statement: 1)... you seem to be trying to discount these feelings.

2)... your response seems to be 'wty want what you don't have.'

3)... telling yourself that you're better off without him, might be your way to feel less anxious.

Referent:

"But then I know, I'm really better off without him."

Scenario \#2

Dynamic:

The wish.

Therapist's statement: I) It seems as though you have a real interest in Richard ....

2) It sounds as though you really like Richand

…

3) It sounds as though you really want Richand

Referent:

…

"Sometimes I think he's exactly what I've been looking for ... now I'm worried that he'll never call me again." 
Dynamic:

Therapist's statement: 1) ... a

1)... a rather unsettling interest ...

2)... that's stirned up some apprehension ...

$3)$... that has aroused your fear of getting hurt.

Referent:

"I started to feel really ugly and dull. I began to wonder what he sees in me. I felt like I was going to start crying... I don't want to get hurt again.

Dynamic:

Defense - Projection, Reaction Formation.

Therapist's Statement: 1) You seem to be trying to disown these feelings.

2) Your response seems to be 'he's not inturested in me.'

3) Claiming that he's really not interested in you sounds like a way of protecting yourself.

Referent:

"hey if you'd rather be with her - fine... Better he break up with me now than later.'

\section{Transference}

\section{Scenario \#1.}

The transference relationship: 1) You seemed to have some difficulty, today, deciding whether to tell me what happened ...

2) Your difficulty and hesitation with me

3)... but also here with me, in your

The current relationship: difficulty in telling me what happened. 1)... the incident in the store was $a$ aifficult one for you.

2)... the difficulty you had deciding what to do when you saw your ex-husband. 3)... this showed up in the store with your ex-husband ...

The link:

1) repetition of the word 'difficulty' when describing the two situations. 2)... makes me think. (again, the repetition of the word 'difficulty." 3) You seem to have difficulty approaching men ... but also here with me 
Scenario \#2.

The transference relationship. 1) You seem to be wondering what I see in you ....

2) Your doubt about being a good patient.

3)... when you wondered if I think you're not making any progress.

The current relationship: $\quad$ I)... the incident in the restaurant has left you wondering about you vorth.

2)... your doubt about being good enough for Richard.

3)... This showed up in the restaurant with Richard.

The link:

1)... repetition of the word "wondering" when describing the two situations.

2)... reminds me of ... (again the repetition of the word 'doubt'). 3) You seem to anticipate rejection ... but also here with me. 
APPENDIX I. Mean and Standard Deviation of PMPP Variables

PMAP Variable

$\mathrm{N}$

Mean

5.96

1.99

4.58

1.91

Speed of

Transference
79

79

79

79

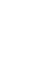

3.38

1.65

2.45

.67

Speed of

Dynamics 
APPENDIX J. Therapist-Rated Patient Characteristics.

Patient Name:

Therapist Name:

Date:

Psychological Mindedness

Iikability

Response to Interpretation 
PSYCHOLOGICAL MINDEDNESS:

This refers to the patient's tendency to reoognize relationships among his (her) internal events (wishes, affecis, defenses, conflicts) as well as interactions between those events and his/her behavior. The use of psychological jargon is not a criterion.

7 Excellent spontaneous demonstration of psychological mindedness.

6

5 Good Demonstration of psychological mindedness with the direction and aid of another member and/or therapist.

4

3 Fair Definite difficulties in making distinctions and/or in links among events.

2

1 Poor Absence of ability and interest in viewing events and problems in this way. 
RESPONSE TO INIERPRETATION:

This refers to the patient's tendency to work with an interpretation. The patient preserves a focus on himself (herself), preserves a focus on the meaning of the interpretation, and adds something important to the interpretation. It is based on abserving the patient's response to interpretations made during the sessions.

7 Excellent There is a spontaneous productive tendency to work with the interpretation.

6

5 Good. With direction and aid from the therapist there is a tendency to work with the interpretation.

4

3 Fair There is minimal work (even with assistance).

2

I Poor There is a clear absence of work*.

* The patient does not engage in work, i.e., he (she) does not add something important to the interpretation. There are many ways of avoiding work by doing something else. They include: remaining silent, requesting clarification, reflecting what the therapist said, changing the object of focus to scmeone else, and changing the topic (content) of frcus. Agreeing with the therapist and/or expressing feeling are not criteria for work. Thus, a patient who merely expresses agreement and enthusiastically reflects what the therapist said is regarded as not working. 


\section{LIKEABIIITY}

This refers to the quality of how likeable, pleasant, and attractive the patient is perceived regarding membership in the group. It refers to how pleasurable it is to work with the patient. It does not concern prognosis, i.e. prabable outcome with therapy.

7 Excellent The patient is very likeable and appealing.

6

5 Good The patient is likeable, but this does not stand out in a dominant way.

4

3 Fair The patient conveys a basically neutral (or ambivalent) impression regarding likeability.

2

1 Poor The patient evidences qualities which the therapist definitely dislikes. 
APFENDIX K. Mamual for the PSychodynamic Work and Object Rating System

\author{
PSYCHODYNAMI: WORK AND OBTECT RATTIKG SYSTEM (FWORS) \\ MANUAT,
}

Introcuction:

The FWORS is a system for rating the level of work engaged in by patients and therapist(s) in psychotherapy groups. According to the system, work is defined as an attempt by a group member to understand the problems of one or more members of the group, or the group as a whole in terms of conflict among dymamic components. Dynamic components are internal forces in the group that are part of a conflict. This means that a dynamic component is assumed to be exerting an internal force on one or more members, or on the group as a whole and that at some level the force is opposed. Excluded from the definition of work are the mere identification or description of resultant (end) states and consideration of dymamic factors that belong to persons or situations external to the group.

Key Words Utilized:

Objects refer to people - inside or outside the group. Units of the Group refer to a patient, the therapist, a dyad, a subgroup, or the group as a whole.

statements are a part of a sentence, a sentence, or several sentences spoken by an internal object which are separated by a statement of another internal object or by a silence greater than 10 seconds (even if the same object contimes speaking)

Resultant Expressions ane affects, cognitions or behaviors that belong to a unit of the group, and that are presented as end states.

Dynamic componernts are wishes (imoulses, motives, drives), reactive anxiety (fears), defensive processes (avoiding, minimizing, resisting), and dynamic expressions that balong to a unit of the group. They are assumed to exert an intermal force on another unit of the group. Dynamic expressions are affects, cognitions and behaviors that are presented as exerting a dynamic influence on a unit of the group.

\title{
Description of the FWORS:
}

There are five components in the system. Four are dynamic and one is nondynamic. The four cyanamic components are wishes, reactive anxiety, defensive processes and dynamic expressions. The nondynamic component is objects. Objects refer to people - inside or outside the group. The PWORS monitors two aspects of objects in each statement: Object Focus and Object Iinking. The five components are used to differentiate four categories of work and nonwork. 


\section{Non-Work Categories:}

\section{Externalizing Statements}

Statements that focus on topics that do not involve a unit of the group and/or focuss on cbjects external to the group. The statement fails to indicate the process in which the external object and the unit of the group are engaging or the impact between the two. The object may be an important figure to a unit of the group.

\section{Descriptive statements}

Statements that provide or request information about a unit of the group. The object focus of the statement may also include an external object. If the information only concerns an abject external to the group, the statement must indicate the interpersonal process in which the external object and a unit of the group are engaging or the impact between the two.

\section{Work Categories:}

3. Single Dynamic Component Statements

statements that provide or request information about a single dymamic component.

4. Multiple Dymamic components Statements

Statements that provide or request information about two or more dymamic components. These components nesd not belong to the saive unit of the group. The relationship between the two dymamics need not be identified in the statement. Hence, the impact of one dysamic on another, or the conflictual nature of the dynamics need only be implied and not explicitly stated.

\section{Explanation of EworS Work Categories}

Category I contains statements that do not involve a unit of the group. Category 2 contains statements that focus on at least one unit of the group but do not identify dymamic components. As categories I and 2 comiain statements that do not attempt to understand the problems of a unit of the group in terms of dymamic ocmponents, they are nonwork categories. Categories 3 and 4 contain statements that focus on at least one unit of the group and identify dymamic components. Hence, categories 3 and 4 contain statements that involve work.

\section{Object Focus}

In addition to rating the level of work engraged in by group members, the Pwors also monitors the object Focus of statements and whether they include object Links. The object Focus refers to whether the speaker is focusing on objects internal or external to the group i.e. the rater notes whom the statement is about. An object Iink refers to a shared interpersonal process between a unit of the group and two objects. 


\section{Rating the Object Focus}

In using the system, each statement is first analyzed for the object focus. This involves three steps.

1) The rater notes who is speaking.

2) The rater notes whom the statement concerns. This involves deciding whether the statement involves objects internal and/or external to the group. The statement may be about internal and external objects. All are noted. If the object focus does not include a unit of the group, a rating of category 1 is given, and the rater proceeds to the next statement.

3) The rater determines whether there are object links. If there is an internal object focus, the rater determines whether there are object links. In this determination, the rater must identify an identical interpersonal process which is cocurring between the previously noted unit of the group and two objects. The two objects which form the link can be either internal and/or external to the group. The unit of the group engaged in the same interpersonal process with the objects is considered "the vortex." The vortex and the Iinked objects are all noted.

II Rating the Ievel of Work

If there is an intemal object focus, the rater proceeds to rate the level of work. Several steps are irrolved.

1. The rater determines whether the statement includes dynamic components:

a) If the statement identifies a wish, a reactive anxiety or a defensive process, the appropriate dynaric component is noted.

b) Determining whether there are any dynamic expressions, is a more complicated task. The rater studies each affective, behavioral, cognitive or vague expression belonging to a unit of the group, and decides whether it is presented as being in conflict with, causing, giving rise to or impacting on another expression of this or any other unit of the group. The appropriate subscripts are noted. The rater must remember that the two aspects of the dymamic expression need not belong to the same unit of the group.

2. If the statement identifies dynamic components, the rater must decide which work category is appropriate.

a) If there is one dynamic component scored, a rating of category 3 is given.

b) if there is one type of dynamic component scored, but there are two or more examples of it, the rater must decide if they are in conflict with each other. If they are not, a category 3 is given. If the two examples of the same type of dynamic component are presented as being in conflict with each other, a rating of category 4 is warranted.

c) If there are two (or more) types of dynamic components 
noted, a rating of category 4 is given. The rater must remember that the dymamic components need not belong to the same unit of the group. The rater then proceeds to the next statement.

3. If there are no dynamic components noted the rater must decide which non-work category is appropriate.

a) If there are no resultant expressions, a category 1 is given. Hence, when a unit of the group is presented as being the recipient of an external object's expressions or dynamics, the statement would be considered an externalization.

b) If there is at least one resultant expression noted, a rating of category 2 is given. The rater proceeds to the next statement.

Explanations, Examples, and Roules

\section{Dynamic Components:}

There are four types of dynamic components: wishes, reactive anxiety, defensive processes and dynamic expressions. To be rated, a dynamic component must belong to a unit of the group.

\section{Notation:}

The dynamic components are indicated by a letter within parentheses:

$\begin{array}{lll}\text { (W) }=\text { Wish } & (A)=\text { Reactive Anxiety } & (D)=\text { Defensive process }\end{array}$

$(E-E)=$ Dymamic Expressions: $($ Ea) $=$ Affective dymanic expression

$(\mathrm{EO})$ = Behavioral dynamic expression

$(E c)=$ Cognitive dymamic expression

$(E v)=$ Vague dynamic expression

With dynamic expressions, the affect, behavior or cognition that is exerting a dymamic influence on a unit of the group is identified with the appropriate subscript. In addition, the expression that is resultant of the dynamic expression is also noted. For example, if an affect is giving rise to a behavior, this would be noted as: (Ea Eb). "Vague" dynamic expressions refer to cases where the absence of an affect, behavior or cognition is presented as exerting a dymamic influence on a unit of the group. Vague expressions are also noted when the rater is unclear how to classify the expression but is certain that it is dynamic.

\section{FWORS Work Categories}

Ratings of categories 3 and 4 depend on the number of types of dynamic components. A repetition of the same type of dynamic component does 
not differentiate the categories. For example, merely identifying three defensive processes in the same statement would be rated category 3 not 4. If, however, the statement presented a conflict between one type of defensive process and another, this would merit a rating of category 4. Statements which provide an antecedent (dynamic) or resultant expression of a wish, a reactive anxiety or a defense do not merit category 4 ratings. Hence, when a dynamic expression contains a wish, a reactive anxiety, or a defense, these latter components would take precedence over the dynamic expression and would not receive a rating of 4. However, when a dymamic component other than a dynamic expression is presented as being an antecedant or resultant of another type of dynamic component, then both dynamics are rated and a category 4 is warranted. The rater is cautioned against confusing descriptions and/or elaborations of one dynamic component with the identification of another.

\section{Defining Dynamic Components}

\section{Wishes:}

To score a wish, the statement must be about the presence of a wish, drive, motive. e.g. "I think you want him back." This would be scored (w) and rated category 3.

If the statement is about the absence of a wish, it can be rated as a dynamic component if it presented as a dynamic expression. e.g. "You don't want to get hurt again so you refused his invitation." In this case it would be scored (EV - Eb). This merits a rating of category 3 with the (Ev) referring to "you don't want to get hurt again..." and the (ED) referring to "...so you refused his invitation."

A dynamic romponent can also be rated if the absence of a wish is presented as being in conflict with a wish. e.g. "You want him back, but on the other hand, you don't want to get hurt again." In this case category 4 is rated with "You want him back" (W) being in conflict with "you don't want to get hurt again (EV).

If a wish is presented as a defensive process in the sense of wanting to defend, the wish is scored. e.g. "You want to avoid your sadness." This merits a rating of 3 for $(W)$.

\section{Reactive Anxiety:}

To score reactive anxiety, the statement must present a fear or anxiety as being a causal agent, a counterdrive (i.e. counter to the wish), in opponsition to a wish, or in reaction to an internal state.

e.g. "It's because you're afraid of getting hurt again that you refused his invitation." This rates a category 3 for the (A) which refers to the fear of getting hurt again which is giving rise to the refusal of the invitation.

e.g. "You want to get involved again but you're scared." Here a rating of 4 is merited for the wish to get involved again (W) is in conflict 
with the fear - of getting involved again - (A).

e.g. "The silence makes you scared." This merits a rating of category 3 for the fear of the silence (A).

\section{Defensive Processes:}

To score a defensive process, the essential idea that must be communicated in the statement is the quality of avoiding, resisting, distorting, being reluctant, etc. e.g. "You're trying to ignore your anger."

components being defended against may be dynamic (wishes, anxieties) or nondynamic (anger, helplessness). e.g. "You're discounting your desire for a new relationship." or "You're discounting your anger." To score defense, however, the statement need not identify what is being defended against nor how the defense manifests itself. e.g. "You're reluctant to talk about that."

When the statement does identify a dynamic component as being defended against, all are soored. e.g. "I think you want another relationship despite how much you keep denying it." This would merit a category 4 rating.

components being defended against may belong to any unit of the group. e.g. "You're reluctant to address her anger at you."

\section{Dynamic Expressions:}

Dynamic expressions are affective, behavioral, cognitive or vague expressions that are presented as exerting a dymamic influence on a unit of the group. To be scored as a dymamic component, the expression must be presented as as being in conflict with, causing, giving rise to, or impacting on another expression of this or another unit of the group. This second expression (resultant) must be stated, and the connection between the dynamic and resultant expression must be clear in the rater's mind. Hence, while an expression may be presented as exerting an influence on a unit of the group, if it presented in a vague and questioming mamer, it would not be rated as a dynamic expression. The rater is cautioned against confusing expressions that occur simultaneously as opposed to sequentially as only the latter can be considered dynamic. While the resultant expression must belong to a unit of the groum, it need not belong to the same unit of the group as the dynamic expression.

e.g. "I think you didn't come last week because you were upset at what I said to you the week before." 3 (EV - Ea), (Ea - EV). The (Eb) refers to "... What I said...", the (Ea) refers to "...you were upset..." and the (Ev) refers to "...you didn't come last week...". The (Eb) is presented as giving rise to the (Ea) which is presented as giving rise to the (Ev). As these two dynamics are the same type of components i.e. dynamic expressions, and they are not presented as being in conflict with one another, a rating of 3 is merited.

e.g. "You don't want him back but you don't want to be alone either." 
This is an example of the aksence of a wish being in conflict with the absence of another wish. (EV) is scored in both cases and a rating of 4 is merited.

e.g. "When you ary like that I feel really guilty, like I upset you or something." 3 (Eb - Fa). (Eb) refers to the patient crying which gives rise to the speaker's feelings of guilt (Ea). A second dynamic expression is not rated for "like I upset you" for it is not seen as giving rise to the crying, but merely acts to clarify what the guilty feelings are about.

\section{$\checkmark$ Defining the Non-Dynamic Component}

The ron-dynamic component of the FWors is objects. Two aspects of objects are rated: Object Focus and Object Links.

The Object Focus refers to whether the speaker is focusing on objects internal or external to the group - i.e. the rater notes whom the statement is about. Objects that are internal to the group are the speaker, or another unit of the group. Objects that are external to the group include general classes of people, specific people known to a unit of the group, units of the group who are absent during the current session arrl members who have left the group. Statements that focus on objects axternal to the groum, may or may not indicate the interpersonal process in which the external object and a unit of the group are engaging or the impact between the two.

Sometimes the focus of the group is ambiguous. A restrictive approach to rating an internal focus is actvocated. To be a focus, the object must be discussed or talked about. Hence, expressions of speech such as "you know" or "I think" are not sufficient to be rated as a focus. As a general rule, if deleting these expressions of speech results in the absence of an internal focus, then an external focus is rated. Similarly, general statements, platitudes or truisms are not assumed to involve a unit of the group. An external focus should be given when substituting "one" for "you" in a statement does not disrupt the meaning of the statement. There is one exception. If a therapist's statement does not identify a specific unit of group, it is assumed that the object focus is the group as a whole rather than people in general. As a general rule, when doubt remains in the rater's mind, an external focus should be given.

objects are scored according to the following abbreviations.

\section{Internal Objects:}

S: Speaker

T: The therapist

SUB: An identified subgroup, (e.g. the men; the women)
D: A dyad

MB: Another specified group member

G: The group as a whole 


\section{Extermal Objects:}

M: Mother

F: Father

P: Parent

SB: Sibling

FM:

Family O: Another specific person $O G$ : A former or absent member of the group EG: A specific group other than this therapy group

$U$ : General classes of people

When an inteipersonal process cocurring between a unit of the group and an object is identified as cocurring in the same way between that same unit of the group and a second object, this constitutes an object link. The shared intexpersonal process may or may not be dymamic. Both of the linked objects may be internal, or both may be external, or one may be internal and one extermal. By definition, the vortex is always internal. The unit of the group at the vortex of the link and the linked objects are all noted in the appropriate columns.

\section{Statements with double foci:}

If a statement has two different foci, the rating priority is given to the higher work rating.

\section{VII statements with double internal object focus:}

If a category 3-4 statement has two internal abjects rated as the focus, the object(s) that refers to the dynamic component is circled. In this way, it can be determined whether the work involved the speaker or another unit of the graup

\section{Hypothet ical expressions, dreams:}

Statements dealing with hypothetical expressions or dream material (and possible imaginary experiences and objects) receive the same rating as any other expression.

IX Questions that involve a dynamic process:

content determines whether or not a statement is rated as identifying dynamic components. To rate dynamis components identified in a question, the following criteria must be met. For defence, reactive anxiety or dynamic expression to be rated, that which is giving rise to the defence, the anxiety, or the resultant expression (respectively), must be presented as being internal to the group, actually occurring, and specifically identified in the intervention or clearly understood from the context. For a wish to be rated, it has to be presented as actually ocaurring and specifically identified in the intervention or clearly understood fram the context.

\section{Facilitative communication:}

Facilitative commication by patients or therapist(s) do no create two separate statements. 


\section{Silence:}

Silence is rated if it is 10 seconds or more. A silence also indicates a new statement. The rater notes the object(s) who initiated and intemuted the silence - e.g. T-sil - T, indirates that the silence cocured between two therapist statements.

XII Timing:

The duration of each statement is timed and noted (in seconds). The rater also times a subsection of the statement. This subsection is the interval that merited a coding of dynamic component and/or resultant expression. Hence, in addition to the overall curation of the statement, the rater also notes the duration that the speaker engaged in descriptive and/or dynamic work. By definition this excludes category 1 .

\section{FWORS Categories and Examples}

\section{Externalizing Statements}

1. The traffic is so heavy today; backed up as far as the eye can see into the parking lot.

\section{1}

2. I think she (absent member) dion't a me back this week because she's scared.

$$
1 \propto G
$$

3. The YWCA was so helpful; the counsellor told everyone what to do - Iike to write a letter to the one who had diec.

$$
\text { I } 0 \text {, EG }
$$

4. My mother was so involved in the church, she'd go there at least twice a week and help out.

$1 \mathrm{M}, \mathrm{EG}$

5. The minister preached the most cnmmy sermon - just doing a Job; he didn't care about her.

$$
\text { I } 0, \mathbf{M}
$$

\section{Descriptive Statements}

1. I was almost late today; I wondered if I would make it at all. $2 \mathrm{~S}$

2. I'm really concerned about sue (absent member); I hope she hasn't gone and done something stupia.

$$
25, \propto G
$$


3. (To the therapist) Are you going to call her (absent member) and make sure she's ok?

$$
2 \mathrm{~T}, \propto \mathrm{G}
$$

4. I always felt that she (Mother) cared more about strangers than she did about me

$$
2 S, M, U
$$

5. Were you really close to your mother?

$$
2 \mathrm{MB}, \mathrm{M}
$$

\section{Single Dynamic Component Statments}

1. I think you're (group member) trying to fool yourself into thinking she's (ex-wife) going to come back

$$
3 \text { (D) } M B, O
$$

2. He (group member) got angry because you (group member) kept asking questions?

$$
3(E B-E a) M B, M B
$$

3. It seems like you (the group in general) want me (the therapist) to take care of you the way you would have wanted the various other people in your lives to take care of you.

$$
3 \text { (W) } T / G / U
$$

4. I think you're (group member) frightened to death to get in touch with how angry you are at him (ex-husband) for leaving you.

$$
3 \text { (A) } \mathrm{MB}, \mathrm{O}
$$

\section{Mutiple Dynamic Components Statements}

1. I was always so afraid when $m$ father would drink that I would just cry and cry. Yet, sometimes I wish I could get that smashed.

$$
4 \text { (A) (W) S, F }
$$

2. Well I'm not going to sit here in silence; not talking about what we're supposed to talk about. Last week I got so angry with you (looks at therapist) for not telling us where sue (member of today's group) was. Are we not supposed to be concerned aixout each other?

$$
4 \text { (D) (EV - Ea) S, G, T, MB }
$$

3. I wish my husband were here; he'd really get things going - he was such a character. I always get so frightened by heing with a group of strangers.

$$
4 \text { (W) (Eb - Ea) G/S/U, O }
$$


4. It's like I don't want to know anything personal about anybody in here and at the same time I feel so lonely that I want to feel I belong somewhere.

$$
4 \text { (Ev) vs (W) S, G }
$$

5. I think members of this gromp are afraid to talk about their feelings and that perhaps it is easier, or safer to sit in silence instead of "opening up the wounds again".

$$
4 \text { (A) (D) } G
$$

6. I think we need more direction than what you're (the therapist) giving us. It makes me so angry when you just sit there and don't say anything; when you don't tell us what we should be doing to get better again. And when you do speak, you just confuse us - just like my dad; he was always speaking over everyone's head - like I didn't deserve an explanation.

4 (W) (EB - Ea) $T / S / F, G$ 
APFENDIX I. Definition of Patient Work Behavior for Therapist Ratings

Definition of Patient work Behavior in STG

In determining your ratings of each patient's level of work in the session, we suggest you consult the following definition of patient work.

When a patient is working in gramp therapy, he takes responsibility for his or the group's problems by exploring causes for them. This exploration irrvolves attempting to explain a problem by causally linking it to internal experiences or behaviors of himself or the group. These internal experiences may relate to motivation (e.g. wishes, fears) behind a certain maladaptive (problematic) bahavior or circumstance. They may also relate to cognitions or affects which are causally linked to a problem. Behaviors presented as giving rise to problems may include defersive manoeurres (e.g. withdrawal, arguments).

A patient who focuses on the (assumed) internal experience or behaviors of other people (extermal to the group) is not engaged in work. Such focussing does not reflect a personal responsibility for problems but rather it implies that his (or the group's) problems are caused by something or sameone external. In addition, a patient is not working when he merely states or describes a problem but does not explore the possible (internal) rasons for it. 


\section{APPENDIX M. Therapist-Rated Process Ratings.}

You are asked to provide an impression (expressed as a percent) for each patient in your group for two variables. Please use your abservations of each patient's behavior during this therapy session.

1. PARIICIPATION. Of the total verbal participation of all the patients, how much did each patient contribute? The total for all patients equals $100 \%$

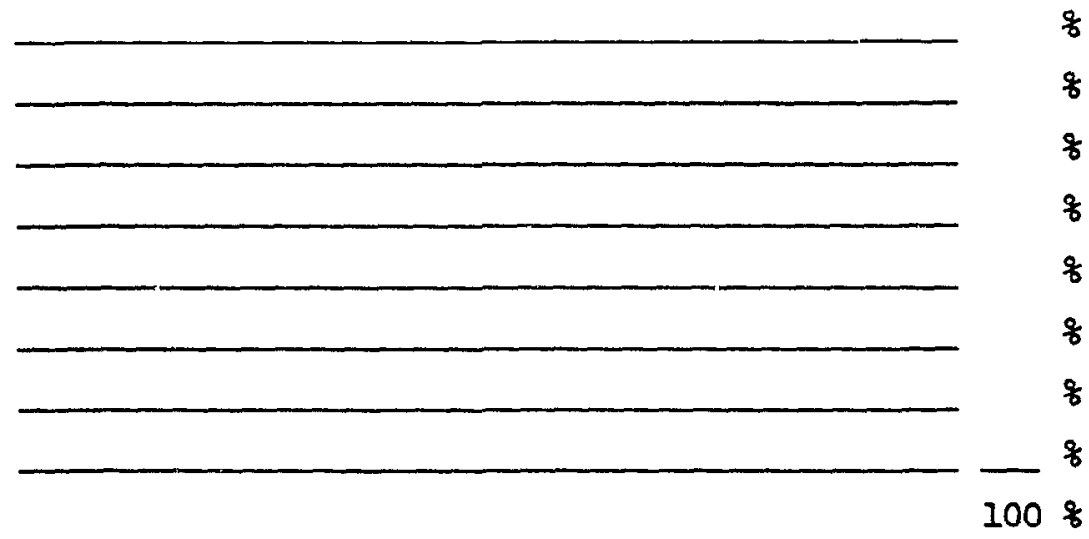

2. PERCENT OF EACH PATIENT'S PARTICIPATION THAT WAS ON-TASK (WORK). Each patient participated a certain amount. For each patient what percent of his/her amount was on-task (work)? The total of the percents for all patients is not expected to equal $100 \%$

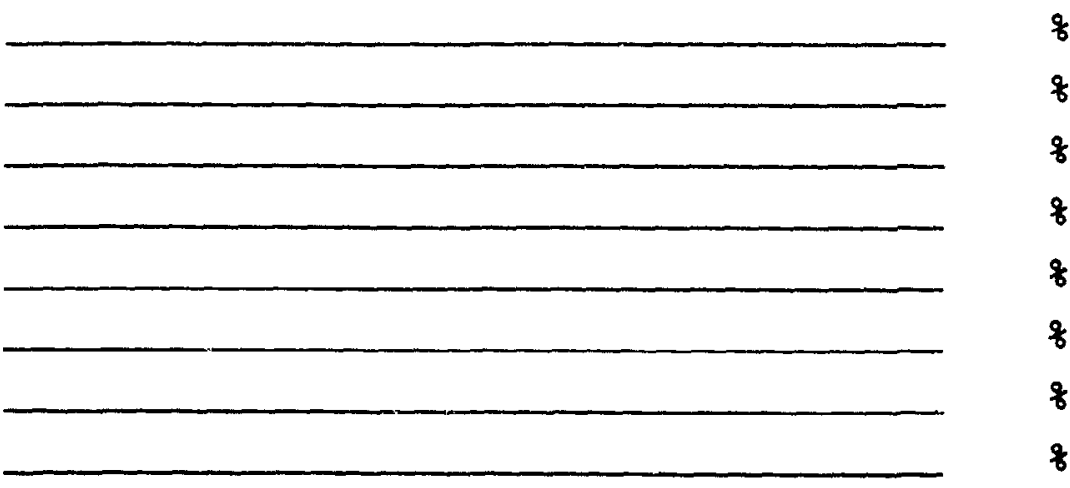


APPENDIX N: Qutcome Measures

\section{A. SOCIAL ADUUSTMENT SCALE}

In responding to the following questions, please answer in consideration of the last month.

WORK $\geq 15$ hours/week $\geq 3$ weeks/ 3 months Students $\geq 1 / 2$ time

Housewives - work less than 15 hours/week

1. Time Lost - How many days/weeks have you missed?

2. Inefficiency - How well have you been doing your work? Do you meet requirements? Get negative feedback?

3. Friction - How much friction has there been between you and your colleagues/boss - how does it show?

4. Disinterest - How interesting have you found your work?

5. Distress - How upset, tense, anxious, have you felt at work? Does it interfere or take a loi to control?

6. Feeling Inadequate - Do you ever feel inferior to others at work? Not doing as well? Does this bother you?

7. Economic Adequacy - Have you had enough money to meet the needs of the family (not postponing essentials or dipping into savings). Can you afford luxuries?

FAMILY OF ORIGIN - if no living relatives - questions 7 and 8

1. Contact - How much contact have you had with members of your family? Telephone conversations/letters?

2. Confiding - How open are you with your family - what do you hold back?

3. Dependency - How mach have you depended on your family for material or emotisnal support?

4. Friction - How much friction has there been between you and your family? How does this come out?

5. Worry - How much have you worried about something happening to members of your family? Does this ocaupy a lot of your time?

6. Defiance - On the other hand, how much have you done out of a wish 
to rebel or make them angry?

7. Guilt - How guilty have you felt about disappointing or letting your family down? How strong is the feeling?

8. Resentment - Otherwise, how resentful have you felt about them disappointing you or letting you down?

SOCIAI AND IEISURE - if no friends, $Q 1,7,8,9,10,11$

1. Contacts - Who are the 2-3 people you have seen socially?

2. Interactions - How much contact have you had with those people? What kind of contact?

3. Feelings - How open are you with these friends? Have you expressed positive and negative feelings?

4. Friction - How mach friction has there been with each of your friends? How does it come out?

5. Hypersensitive - How sensitive have you been about what your friends have said about you? Do you over-react?

6. Distress - How relaxed or confortable do you feel with these friends? Do you feel tense, anxious, avoid them?

7. Need for contace - Have you felt the need for either more friends or more contact with the friends you have?

8. Impaired Ieisure Activities - Have you lost interest in your usual hobbies or leisure activities?

9. Boreciom - Have you felt bored in your free time? often?

10. Dating - How often have you dated?

11 Interest in Dating - Are you still interested in dating or has this diminished?

PARINER - Regular contact with spouse of at least 8 hours/week.

1. Confiding - How open are you with your partner (positive and negative feel.ings)? What do you hold back?

2. Friction - How much friction has there bein between you and your partner? How does this come out?

3. Dependency - How dependent have you been on your partner? Does this trouble you? 
4. Submissiveness - How submissive have you been (giving in)?

5. Domineering - How dowineering have you been? Making decisions without consulting/considering partner?

6. Need For warmth - How much have you felt the need for more affection and/or attention from your partner?

- Worry - How much have you worried about something happening to your partner? Are you troubled by this?

8. Guilt - Have you felt guilty about letting your partner down or being unfair to him/her? Is this strong?

9. Resentment - Have you felt resentful that your partner has let you down, been unfair to you?

\section{SEXUAL FUNCIIONING -}

1. Frequency - Approximately how often have you had sexual relations curing the last three months?

2. Physical Problems - Have you experienced any physical problems with sex? How often - does it interfere or prevent you from having sex?

3. Satisfaction - How often have you been able to relax and enjoy sex? How satisfying is sex sor you?

MARTTAL AS PARENT - minimm of 8 hours/week.

1. Responsibilities - How would you define your responsibilities to your children during the last 3 months? To what extent have you been fulfilling them?

2. Communication - Are you able to discuss things with your children (do they open up)? What do you hold back?

3. Friction - How much friction has there been between you and your children? How does this come cut?

4. Need For warmth - How often have you felt the need for more affection or attention from them? Is it strong?

5. Worry - How mach have you worried about something happening to your chilibren? Does this cocung a lot of your time?

6 Guilt - Have you felt guilty for letting your children down or being unfair to them/him/her? How much?

7. Resentment - on the other hand, have you felt resentful that they have let you down or been unfair to you? 


\title{
B. TARGET OBJECTIVES
}

\author{
OBTECTIVES
}

Consider each of the objectives that have been formulated. You are asked to do three different things with each objective.

First, indicate how severe (disruptive) the problem associated with each objective has been for you during the last month by placing a number in the appropriate colum according to the following:

$\begin{array}{ccccc}1 & 2 & 3 & 4 & 5 \\ \text { slight } & \text { minor } & \text { moderate } & \text { considerable } & \text { extreme } \\ \text { severity } & \text { severity } & \text { severity } & \text { severity } & \text { severity }\end{array}$

second, indicate how important each objective is to you by placing a number in the appropriate column, acoording to the followilg:

$\begin{array}{ccccc}1 & 2 & 3 & 4 & 5 \\ \text { slight } & \text { minor } & \text { moderate } & \text { considerable } & \text { extreme } \\ \text { importance } & \text { importance } & \text { importance } & \text { importance } & \text { importance }\end{array}$

Remember, severity and importance refer to two different things (e.g., a probleri may be of considerable severity but only of minor importance to you, or a problem may only be of minor severity, yet be of extreme importance to youl).

Third, indicate how much improvement you expect for each problem by the end of psychotherapy, by placing a number in the appropriate colum, according to the following:

$\begin{array}{ccccc}1 & 2 & 3 & 4 & 5 \\ \text { slight } & \text { minor } & \text { moderate } & \text { considerable } & \text { extreme } \\ \text { improvement } & \text { improvement } & \text { improvement } & \text { improvement } & \text { improvement }\end{array}$


POST-RAIT ASSESSMENT OF OBUECTIVES

Consider each of the objectives that have been formulated. You are asked to do four different things with each objective.

First, indicate how severe (dismuptive) the problem associated with each objective has been for you during the last month by placing a number in the appropriate colum according to the following:

$\begin{array}{ccccc}1 & 2 & 3 & 4 & 5 \\ \text { slight } & \text { minor } & \text { moderate } & \text { considerable } & \text { extreme } \\ \text { severity } & \text { severity } & \text { severity } & \text { severity } & \text { severity }\end{array}$

second, indicate how important each objective is to you by placing a number in the appropriate column, according to the following:

\begin{tabular}{|c|c|c|c|c|}
\hline $\begin{array}{l}\text { I } \\
\text { slight } \\
\text { importance }\end{array}$ & $\stackrel{2}{\text { minor }}_{\text {importance }}$ & $\begin{array}{c}3 \\
\text { moderate } \\
\text { importance }\end{array}$ & $\begin{array}{c}4 \\
\text { Considerable } \\
\text { importance }\end{array}$ & $\begin{array}{c}5 \\
\text { extreme } \\
\text { importanc }\end{array}$ \\
\hline
\end{tabular}

Remember, severity and importance refer to two different things (e.g., a problem may be of considerable severity but only of minor importance to you, or a problem may only be of minor severity, yet be of extreme importance to you).

Third, indicate how mach inprovement you expect for each problem by the end of psychotherapy, by placing a muber in the appropriate colum, according to the following:

$\begin{array}{ccccc}1 & 2 & 3 & 4 & 5 \\ \text { slight } & \text { minor } & \text { moderate } & \text { considerable } & \begin{array}{c}\text { extreme } \\ \text { improvement }\end{array} \\ \text { improvement } & \text { improvement } & \text { improvement } & \text { improvement }\end{array}$

Fourth, indicate the type of change that ocoured for $\epsilon$ ach problem during the last month by placing a number in the appropriate colum, according to the following:

\begin{tabular}{|c|c|c|c|c|c|c|}
\hline $\begin{array}{c}1 \\
\text { extreme } \\
\text { worsening }\end{array}$ & $\begin{array}{l}2 \\
\text { considerable } \\
\text { worsening }\end{array}$ & $\begin{array}{l}3 \\
\text { moderate } \\
\text { worsening }\end{array}$ & & $\begin{array}{l}\text { or } \\
\text { ening }\end{array}$ & $\begin{array}{l}5 \\
\text { slight } \\
\text { worsening }\end{array}$ & $\begin{array}{c}6 \\
\text { no } \\
\text { change }\end{array}$ \\
\hline $\begin{array}{l}7 \\
\text { slight } \\
\text { mprovement }\end{array}$ & $\begin{array}{c}8 \\
\text { minor } \\
\text { improvement }\end{array}$ & $\begin{array}{c}9 \\
\text { moderat } \\
\text { improven }\end{array}$ & & $\begin{array}{r}10 \\
\text { Consi } \\
\text { impro }\end{array}$ & $\begin{array}{l}\text { derable } \\
\text { vement }\end{array}$ & $\begin{array}{l}11 \\
\text { extreme } \\
\text { mprovement }\end{array}$ \\
\hline
\end{tabular}




\section{POST-IREATMENT ASSESSMENT OF OBJECITVES}

Please consider each of the following objectives that were formulated before therapy began. You are asked to do three different things with each abjective.

First, indicate how severe (disruptive) the problem associated with each objective has been for you during the last month by placing a number in the appropriate colum according to the following:

$\begin{array}{cccccc}0 & 1 & 2 & 3 & 4 & 5 \\ \text { no } & \text { slight } & \text { minor } & \text { moderate } & \text { considerable } & \text { extreme } \\ \text { severity } & \text { severity } & \text { severity } & \text { severity } & \text { severity } & \text { severity }\end{array}$

Second, indicate the type of change that occured for each problem since therapy began by placing a number in the appropriate colum, according to the following:

\begin{tabular}{|c|c|c|c|c|c|}
\hline $\begin{array}{l}1 \\
\text { extreme } \\
\text { worsening }\end{array}$ & $\begin{array}{c}2 \\
\text { considerable } \\
\text { worsening }\end{array}$ & $\begin{array}{l}3 \\
\text { moderate } \\
\text { worsening }\end{array}$ & $\begin{array}{l}4 \\
\text { minor } \\
\text { worsening }\end{array}$ & $\begin{array}{l}5 \\
\text { slight } \\
\text { worsening }\end{array}$ & $\begin{array}{c}6 \\
\text { no } \\
\text { change }\end{array}$ \\
\hline $\begin{array}{c}7 \\
\text { slight } \\
\text { improvement }\end{array}$ & $\begin{array}{l}8 \\
\text { minor } \\
\text { improvement }\end{array}$ & $\begin{array}{c}9 \\
\text { moderace } \\
\text { improvement }\end{array}$ & $\begin{array}{c}10 \\
\text { considerable } \\
\text { inprovement }\end{array}$ & $\begin{array}{r}11 \\
\text { extre } \\
\text { impror }\end{array}$ & $\begin{array}{l}\text { me } \\
\text { ement }\end{array}$ \\
\hline
\end{tabular}

Third, indicate how important (relevant) each abjective is to you by placing a muber in the appropriate colum, according to the following:

$\begin{array}{cccccc}0 & 1 & 2 & 3 & 4 & 5 \\ \text { no } & \text { slight } & \text { minor } & \text { moderate } & \text { considerable } & \begin{array}{c}\text { extreme } \\ \text { importance }\end{array} \\ \text { importance importance } & \text { importance } & \text { importance } & \text { importance }\end{array}$

Remember, severity and importance refer to two different things, (e.g., a problem may be of considerable severity but only of minor importance to you, or a problem may only be of minor severity, yet be of extreme importance to you). 
One of the losses in your life that you have described is people after such a stressful life event. Please read each item, indicating how frecpuently these corments were true for you curring the past seven days. If they did not occur during that time, please mark the "not at all" column. I I thought about it when I didn't mean to. $\mathrm{x}$

Not at Rarely

2 I avoided letting myself get upset when

I thought about it or was reminded of it. $x$

3 I tried to remove it from memory.

4 I had trouble falling asleep or staying asleep, because of pictures or thoughts about it that came into my mind.

5 I had waves of strong feelings about it.

6 I had dreams about it.

7 I stayed away from reminders of it.

8 I felt as if it hadn't happened or it wasn't real.

9 I tried not to talk about it.

10 pictures about it popped into my mind.

11 other things kept making me think about it.

12 I was aware that I still had a lot of feelings about it, but I didn't deal with them.

13 I tried not to think about it

I4 AnY reminder brought back feelings about it.

15 feelings about it were kind of mmb.

$\begin{array}{cccc}X & X & X & X \\ X & x & x & x\end{array}$

X $\quad \mathrm{X} \quad \mathrm{x}$

$\mathrm{X}$ X $\mathrm{X} \quad \mathrm{X}$

$\mathrm{X}$ X $\mathrm{X} \quad \mathrm{X}$

$\mathrm{X} x \quad \mathrm{X}$ X

$\mathrm{X} \quad \mathrm{X} \quad \mathrm{X} \quad \mathrm{X}$

$\begin{array}{llll}x & x & x & x\end{array}$

X $\mathrm{X} \quad \mathrm{X} \quad \mathrm{X}$

X $\quad \mathrm{x} \quad \mathrm{x}$

X $\mathrm{X}$ X

X $\mathrm{X}$ X

X $\mathrm{X} \quad \mathrm{X}$ X

X $\mathrm{X}$ X $\mathrm{X}$




\section{TIIERPPERSONAL DEPENDENCY TIVENTORY}

INSTRUCIIONS: 32 statements are presented below. Please read each one and decide whether or not it is characteristic of your attitudes, feelings, or behavior. Then assign a rating to every statement, using the values given below:

$$
\begin{aligned}
& 4=\text { very characteristic of me } \\
& 3=\text { quite characteristic of me } \\
& 2=\text { somewhat characteristic of me } \\
& 1=\text { not characteristic of me }
\end{aligned}
$$

1. I prefer to be myself.

2. I do my best work when I know it will be appreciated

3. I can't stand being fussed over when I'm sick.

4. I believe people could do a lot more for me if they wanted to.

5. As a child, pleasing $m y$ parents was very important to me.

6. I don't need other people to make me feel good.

7. Disapproval by someone I care about is very painful for me.

8. I'm the only person I want to please.

9. The idea of losing a close friend is terrifying to me.

- 10. I rely only on myself.

- 11. I would be completely lost if I didn't have someone special.

12. I get upset when someone discovers a mistake I've made.

13. I hate it when people offer me sympathy.
14. I easily get discouraged when I don't get what I need from others

15. I don't need mach from people.

16. I must have one person who is very special to me

17. When I am sick, I prefer that my frierds leave me alone.

18. I'm never happier than when I've done a good job.

19. I am willing to disregard other people's feelings in order to accomplish something that's important to me

20. I need to have one person who puts me above all others.

21. I don't need anyone.

22. I tend to imagine the worst if a loved one doesn't arrive when expected.

23. Even when things go wrong I can get along without asking for help from my friend

24. I tend to expect too much fram others. 
25. I tend to be a loner.

26. I feel that I never really get all that I need from people.

27. Even if most people turned against me, I could still go if someone I love stood by me.

28. What people think of me doesn't affect how I feel.

29. I think that most people don't realize how easily they can hurt me.

30. I have always had a terrible fear that I will lose the love and support of people I desperately need.

31. I would feel helpless if deserted by someone I love.

32. What other people say doesn't bother me. 


\section{E. INTERPEERSORAI BEHAVIOR SCALE}

Part I: Please indicate the extent to which each of the following occurs eresently in your life with the people whom you interact with regularly. Such people often include friends, social and work (or sciool) associates. You are asked to state an average for each item by circling the appropriate number.
6. Very frequently
5. Frequently
4. Often
3. Sometimes
2. Seldomly
1. Very seldomly

1. I share personal information with them.

2. They stare personal information with me.

3. I behave freely with them.

4. They behave freely with me.

5. I express $m y$ feelings to them.

6. They express their feelings to me.

7. I communicate clearly to them.

8. They communicate clearly to me.

$\begin{array}{llllll}1 & 2 & 3 & 4 & 5 & 6\end{array}$

9. I express my thoughts to them.

10. They express their thoughts to me.

11. I an honest to them.

12. They are honest with me.

13. I am aware of how I influence them.

$\begin{array}{llllll}1 & 2 & 3 & 4 & 5 & 6\end{array}$

$\begin{array}{llllll}1 & 2 & 3 & 4 & 5 & 6\end{array}$

14. I am aware of how they infiluence me.

$\begin{array}{llllll}1 & 2 & 3 & 4 & 5 & 6\end{array}$

$\begin{array}{llllll}1 & 2 & 3 & 4 & 5 & 6\end{array}$

$\begin{array}{llllll}1 & 2 & 3 & 4 & 5 & 6\end{array}$

$\begin{array}{llllll}1 & 2 & 3 & 4 & 5 & 6\end{array}$

$\begin{array}{llllll}1 & 2 & 3 & 4 & 5 & 6\end{array}$

$\begin{array}{llllll}1 & 2 & 3 & 4 & 5 & 6\end{array}$

$\begin{array}{llllll}1 & 2 & 3 & 4 & 5 & 6\end{array}$

$\begin{array}{llllll}1 & 2 & 3 & 4 & 5 & 6\end{array}$

$\begin{array}{llllll}1 & 2 & 3 & 4 & 5 & 6\end{array}$

$\begin{array}{llllll}1 & 2 & 3 & 4 & 5 & 6\end{array}$

15. I let them know when I am irritated with them

$\begin{array}{llllll}1 & 2 & 3 & 4 & 5 & 6\end{array}$

$\begin{array}{llllll}1 & 2 & 3 & 4 & 5 & 6\end{array}$

16. They let me know when they are irritated with me

$\begin{array}{llllll}1 & 2 & 3 & 4 & 5 & 6\end{array}$

17. I trust them.

$\begin{array}{llllll}1 & 2 & 3 & 4 & 5 & 6\end{array}$

18. They trust me.

$\begin{array}{llllll}1 & 2 & 3 & 4 & 5 & 6\end{array}$ 
19. I tell them how I see them.

20. They tell me how they see me.

21. I am sensitive to their feelings.

22. They are sensitive to my feelings.

23. I am helpful to them.

24. They are helpful to me.

25. I let them know when I have positive feelings toward them.

26. They let me know when they have positive feelings towards me.

27. I am accepting of them.

28. They are accepting of me.

29. I feel satisfied with my relationships with them.

30. They feel satisfied with their relationship with me. $\begin{array}{llllll}1 & 2 & 3 & 4 & 5 & 6\end{array}$

$\begin{array}{llllll}1 & 2 & 3 & 4 & 5 & 6\end{array}$

$\begin{array}{llllll}1 & 2 & 3 & 4 & 5 & 6\end{array}$

$\begin{array}{llllll}1 & 2 & 3 & 4 & 5 & 6\end{array}$

$\begin{array}{llllll}1 & 2 & 3 & 4 & 5 & 6\end{array}$

12535456

$\begin{array}{llllll}1 & 2 & 3 & 4 & 5 & 6\end{array}$

$\begin{array}{llllll}1 & 2 & 3 & 4 & 5 & 6\end{array}$

$\begin{array}{llllll}1 & 2 & 3 & 4 & 5 & 6\end{array}$

$\begin{array}{llllll}1 & 2 & 3 & 4 & 5 & 6\end{array}$

$\begin{array}{llllll}1 & 2 & 3 & 4 & 5 & 6\end{array}$

$\begin{array}{llllll}1 & 2 & 3 & 4 & 5 & 6\end{array}$ 
Part II: Please indicate the extent to which you would like each of the following to cocur in your life with the people whom you interact with regularly. Ayain, you are asked to state an average for each item by circling the appropriate number.

$$
\begin{aligned}
& \text { 6. Very frequently } \\
& \text { 5. Frequently } \\
& \text { 4. Often } \\
& \text { 3. Sometimes } \\
& \text { 2. Seldamly } \\
& \text { 1. Very seldomly }
\end{aligned}
$$

1. I share personal information with them.

2. They share personal information with me.

3. I behave freely with them.

4. They behave freely with me.

5. I express my feelings to them.

6. They express their foclings to me.

7. I conmunicate clearly to them.

8. They commicate clearly to me.

9. I express $\mathrm{my}$ thoughts to them.

$\begin{array}{llllll}1 & 2 & 3 & 4 & 5 & 6\end{array}$

10. They express their thoughts to me.

11. I am honest to them.

12. They are honest with me.

13. I am aware of how I influence them.

14. I am aware of how they influence me.

$$
\begin{array}{llllll}
1 & 2 & 3 & 4 & 5 & 6
\end{array}
$$

$\begin{array}{llllll}1 & 2 & 3 & 4 & 5 & 6\end{array}$

$\begin{array}{llllll}1 & 2 & 3 & 4 & 5 & 6\end{array}$

$\begin{array}{llllll}1 & 2 & 3 & 4 & 5 & 6\end{array}$

$\begin{array}{llllll}1 & 2 & 3 & 4 & 5 & 6\end{array}$

$\begin{array}{llllll}1 & 2 & 3 & 4 & 5 & 6\end{array}$

$\begin{array}{llllll}1 & 2 & 3 & 4 & 5 & 6\end{array}$

$\begin{array}{llllll}1 & 2 & 3 & 4 & 5 & 6\end{array}$

$\begin{array}{llllll}1 & 2 & 3 & 4 & 5 & 6\end{array}$

$\begin{array}{llllll}1 & 2 & 3 & 4 & 5 & 6\end{array}$

$\begin{array}{llllll}1 & 2 & 3 & 4 & 5 & 6\end{array}$

123456

$\begin{array}{llllll}1 & 2 & 3 & 4 & 5 & 6\end{array}$

15. I let them know when I an irritated with them

123456

16. They let me know when they are irritated with ne

17. I trust them.

18. They trust me.

i9. I tell them how I see them.

$\begin{array}{llllll}1 & 2 & 3 & 4 & 5 & 6 \\ 1 & 2 & 3 & 4 & 5 & 6\end{array}$


20. They teil me how they see me.

21. I am sensitive to their feelings.

22. They are sensitive to my feelings.

23. I am helpful to them.

24. They are helpful to me.

25. I let them know when I have positive feelings toward them.

26. They iet me know when they have positive feelings towards me.

27. I am accepting of them.

28. They are accepting of me.

29. I feel satisfied with my relationships with them.

30. They feel satisfied with their relationship with me. $\begin{array}{llllll}1 & 2 & 3 & 4 & 5 & 6 \\ 1 & 2 & 3 & 4 & 5 & 6 \\ 1 & 2 & 3 & 4 & 5 & 6 \\ 1 & 2 & 3 & 4 & 5 & 6 \\ 1 & 2 & 3 & 4 & 5 & 6\end{array}$

$\begin{array}{llllll}1 & 2 & 3 & 4 & 5 & 6\end{array}$

$\begin{array}{llllll}1 & 2 & 3 & 4 & 5 & 6\end{array}$

$\begin{array}{llllll}1 & 2 & 3 & 4 & 5 & 6\end{array}$

$\begin{array}{llllll}1 & 2 & 3 & 4 & 5 & 6\end{array}$

$\begin{array}{llllll}1 & 2 & 3 & 4 & 5 & 6\end{array}$

$\begin{array}{llllll}1 & 2 & 3 & 4 & 5 & 6\end{array}$ 


\section{F. ROSENBERG SELF-ESTEEM SCALE}

Ten items are presented below. Please indicate how strongly you agree or disagree with each item by circling the appropriate alternative.

$$
\begin{aligned}
\mathrm{SA} & =\text { Strongly agree } \\
\mathrm{A} & =\text { Agree } \\
D & =\text { Disagree } \\
\mathrm{SD} & =\text { Strongly disagree }
\end{aligned}
$$

1. On the whole, I am satisfied with myself.

SA $\quad$ A $\quad$ D $\quad S D$

2. At times I think I am no good at all.

SA $\quad$ A $\quad$ D $\quad S D$

3. I feel that I have a muber of good cualities.

SA A

D SD

4. I am able to do things as well as most other people.

SA $\quad$ A $\quad$ D $\quad$ SD

5. I feel I do not have much to be proud of.

SA $\quad$ A $\quad$ D $\quad$ SD

6. I certainly feel useless at times.

SA $\quad$ A $\quad$ D $\quad S D$

7. I feel that I'm a person of worth, at least on an equal plane with others.

8. I wish I could have more resper,t for myself.

SA $\quad A \quad D \quad S D$

9. All in all, I am inclined to feel that I am a failure.

$S A \quad A \quad D \quad S D$

10. I take a positive attitude toward myself.

SA

SA A D SD

SA A $\quad$ D $\quad$ SD 


\section{G. SEIF-REPORT SYMPIOM INVENTORY (SCI-90)}

Below is a list of problems that people sometimes have. Read each one carefully and choose one of the numbers that best describes HOW MUCH THE PROBLEM HAS BOTHERED YOU DURTNG THE PAST WEEK, INCIUDING TOLAY. Place the number to the right of the complaint. Do not skip any items. The exarmple below will show you exactly how to mark your answer. If you have any questions, please ask.

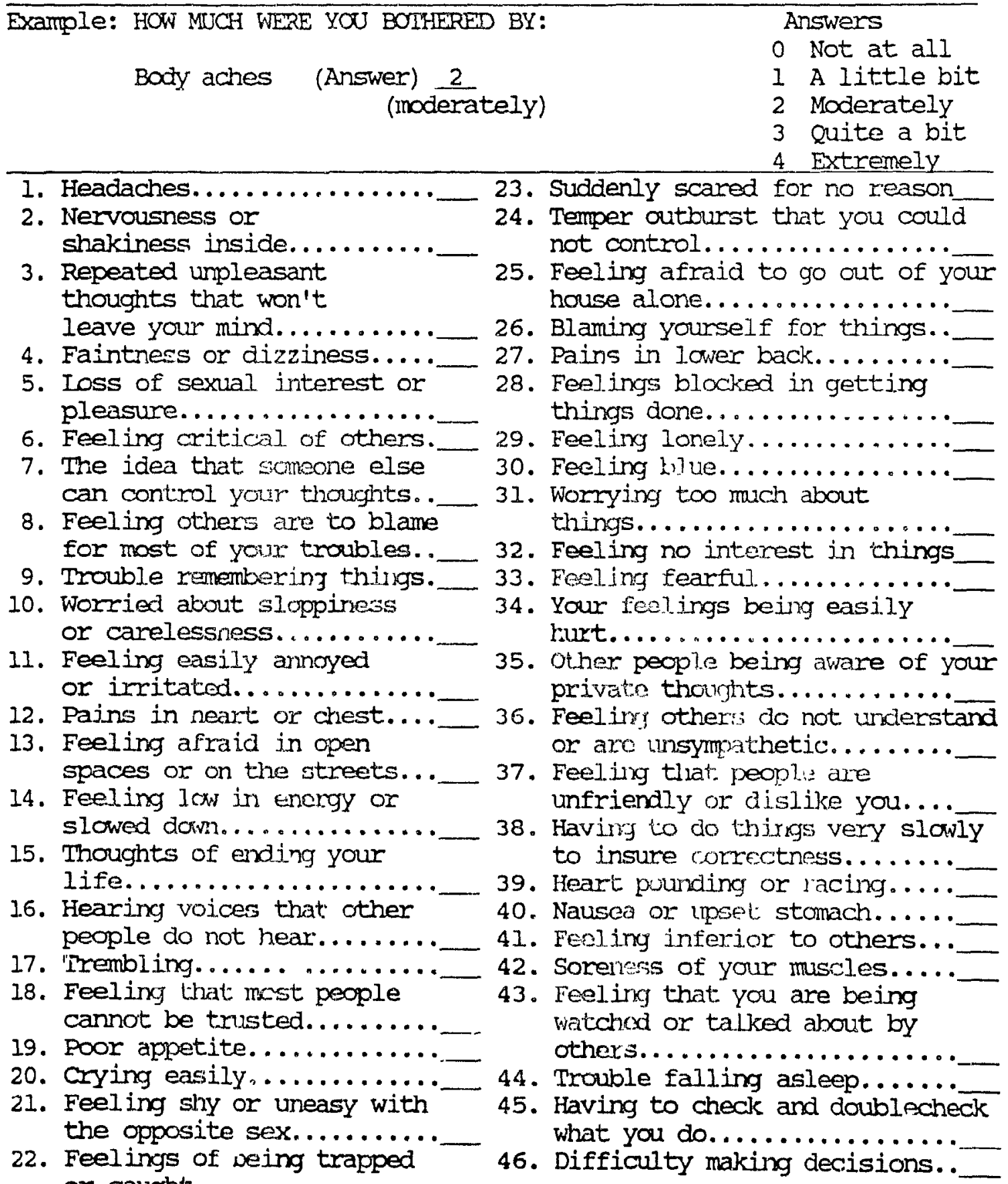




\begin{tabular}{ll}
\hline Question: & Answers \\
HOW MUCH WERE YOU BOTHERED BY: & 0 Not at all \\
& 1 A little bit \\
& 2 Moderately \\
& 3 Quite a bit \\
& 4 Extremely \\
\hline
\end{tabular}

47. Feeling afraid to travel on buses, subways or trains. . . .

48. Trouble getting your breath................

49. Hot or cold spells.........

50. Having to avoid certain things, 75 places or activities because they frighten you..........

51. Your mind going blank......

52. Numbness or tingling in parts

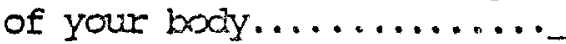

53. A lump in your throat......

54. Feeling hopeless about the future..................

55. Trouble concentrating......

56. Feeling weak in parts of your body...................

57. Feeling tense or keyed up..

58. Heavy feelings in your arms or leys.................

59. Thoughts of death or dying.

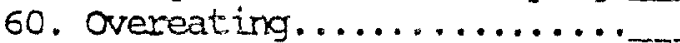

61. Feeling uneasy when people are watching you..............

62. Having thoughts that are not your own................

63. Havirg urges to beat, injure or hanm someone. ...........

64. Awakening in the early morning................

65. Having to repeat the same actions, such as touching, counting, washing..........

66. Sleep that is restless or disturbed...............

67. Having uxrje:s to break or smash things.

68. Having ideas or beliefs that others do not share........

69. Feeling very self-conscious with others.............

70. Feeling uneasy in crowts, such as shopping or at a movie.. .

71. Feeling everything is an effort.................
72. Spells of panic or terror....

73. Feeling uncomfortable about eating or drinking in public.

74. Getting into frequent argumerits...............

Feeling nervous when you are left alone.................

76. Others not giving you uroper credit for your achievements.

77. Feeling Ionely even when you are with people................

78. Feeling so restless you can't sit still................

79. Feelings of worthlessness....

80. The feeling that something bad is going to happen to you....

81. Shouting or throwing things..

82. Feeling afraid you will faint

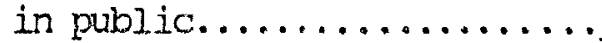

83. Feeling that people will take actvantage of you if you let them....................

84. Having thoughts about sex that bother you alot.............

85. The idea that you should be punished for your sins.......

86. Thoughts and images of a frighterning nature. ..........

87. The idea that something serious is wrong with your body......

88. Never feeling close to another person. ..................

89. Feelings of guilt..........

90. The idea that something is wrong with your mind............. 


\section{H. BECK DEPRESSION INVENTORY}

This questionnaire consists of 13 groups of statements. Please read the entire group of statements in each category. Pick out the one statement in that group which best describes the way you feel today; that is, right now. Circle the letter beside the statement you have chosen. If several statements in the group seem to apply equally well, circle each one.

Be sure to read all the statements ir oach group before making your choice.

1. A. I do not feel sad.

B. I feel sad or blue.

C. I an sad or blue all the time and I can't: snap out of it.

D. I am so sad or unhappy that I can't stand it.

2. A. I an not particularly pessimistic or discouraged about the future.

B. I feel discouraged about the future.

C. I feel I have nothing to look forward to.

D. I feel that the future is hopeless and that things cannot improve.

3. A. I do not feel like a failure.

B. I feel I have failed more than the average person.

C. As I look back on my life, all I can see is a lot of failures.

D. I feel I am a complete failure as a person (parent, husband, wife).

4. A. I am not particularly dissatistied.

B. I don't enjoy things the way I used to.

C. I don't get satisfaction out of: anything anymore.

D. I am dissatisfied with everything.

5. A. I don't feel particularly guilty.

B. I feel bad or urworthy a good part of the time.

C. I feel quite quilty

D. I feel as though I am very bad or worthless.

6. A. I don't feel disappointed in myself.

B. I am disappointed in myself.

C. I am disgusted with mrself.

D. I hate myself.

7. A. I don't have thoughts of harming myself.

B. I feel I would be better off dead.

C. I have definite plans about committing suicide.

D. I would kill myself if I had a chance. 
8. A. I have not lost interest in other people.

B. I am less interested in other people than I used to be.

C. I have lost most of my interest in other people and have little feeling for them.

D. I have lost all of my interest in other people and don't care about them at all.

9. A. I make decisions about as well as ever.

B. I try to put off making decisions.

C. I have great difficulty in making decislons.

D. I can't make any decisions at all anyore.

10. A. I don't feel I look any worse than I used to.

B. I am worried that I an looking old or unattractive.

C. I feel that there are permanent changes in my appearance and they make me look unattractive.

D. I feel that I am ugly or repulsive looking.

11. A. I can work about as well as before.

B. It takes extra effort to get started at doing something.

C. I have to push myself very hand to do anything.

D. I can't do arry work at ali.

12. A. I don't get any more tired than usual.

B. I get tired more easily than I used to.

C. I get tired from doing arything.

D. I get too tired to do anything.

13. A. My appetite is no worse than usual.

B. MY appetite is not as good as it used to be.

C. My appetite is much worse now.

D. I have no appetite at all anymore. 


\section{GLOBAL RATINGS OF OUTCOME}

Part I: Iife Satisfaction Scale

All things considered, how satisfied or dissatisfied are you with your life as a whole these days? Circle one muber on the line that you feel best represents your level of satisfaction with your present life.

Completely completely

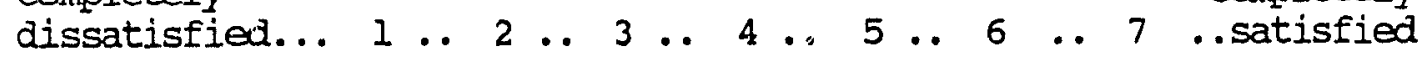

Part II: Overall Usefulness of Therapy

Patient-rated: "Overall, how much was your therapy useful to you?" Therapist-rated: "Overall, how much was therapy useful to each of the following patients?"

$\begin{array}{lllllll}1 & 2 & 3 & 4 & 5 & 6 & 7 \\ \text { Very little } & & & & & & \text { very much }\end{array}$

Part III: Service Evaluation Questionnaire

You can help us improve our program by answering the following questions about the services you have received. We are interested in your honest opinion, whether it is positive or negative. Please answer all the questions. We also welcome your comments and suggestions. circle the number which you feel best represents your opinion.

1. How would you rate the quality of service you have received?

$$
\begin{array}{cccc}
4 & 3 & 2 & 1 \\
\text { Excellent } & \text { cood } & \text { Fair } & \text { Foor }
\end{array}
$$

2. Did you get the kind of service you wanted?

No, definitely not No, not $\stackrel{2}{1}$ really Yes, generally Yes, definitely

3. To what extent has our program met your needs?

4

Almost all needs have been met
3

Most of my needs have been met
${ }^{2}$ only a few needs have been met
1 None 
4. If a friend were in need of similar help, would you recommend our program to him or her?

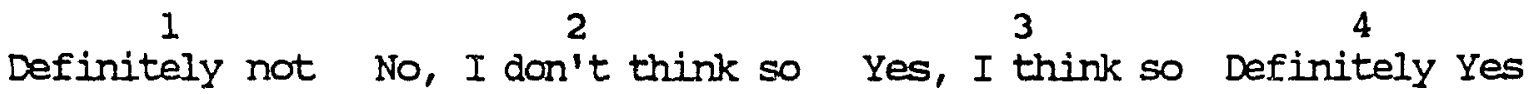

5. How satisfied are you with the amount of help you have received?

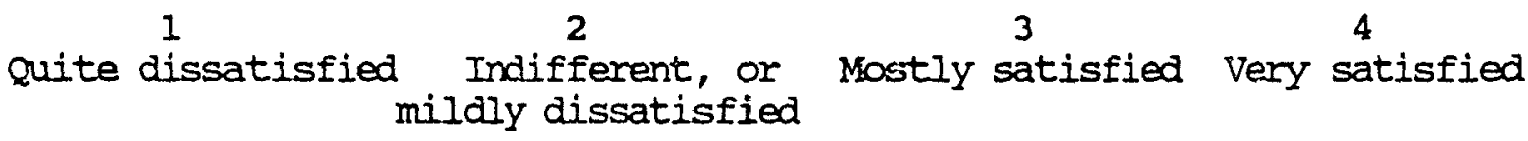

6. Have the services you received helped you to deal more effectively with your problems?

$\begin{array}{cccc}4 & 3 & 2 & 1 \\ \text { Yes, a } & \text { Yes, they helped } & \text { No, they really } & \text { No, they seemed to } \\ \text { great deal } & \text { scmewhat } & \text { didn't help } & \text { make things worse }\end{array}$

7. In an overall, general sense, how satisfied are you with the service you have received?

4
Very satisfied Mostly satisfied $\begin{gathered}2 \\ \text { Indifferent, or quite satisfied }\end{gathered}$ mildy dissatisfied

8. If you were to seek help agair, would you return to our program?

1234

Definitely not No, I don't think so Yes, I think so Definitely Yes 
APEENDIX 0. $2 \times 2$ ANCOVA Tables: Main and Interactive Effects of Ireatment and condition on outcome

Variable 1: Social Adjustment scale - work

$\begin{array}{lrrrrr}\text { Source } & \text { SS } & \text { DF } & \text { MS } & \text { F } & \underline{\text { P }} \\ \text { Within Cells } & 84.53 & 32 & 2.64 & & \\ \text { Regression } & 16.54 & 1 & 16.54 & 6.26 & .018 \\ \text { Constant } & 12.52 & 1 & 12.52 & 4.74 & .037 \\ \text { Treatment } & .60 & 1 & .60 & .23 & .637 \\ \text { Condition } & 1.77 & 1 & 1.77 & .67 & .420 \\ \text { Treatment by condition } & .32 & 1 & .32 & .12 & .731\end{array}$

Variable 2: Social Adjustment Scale - Social

\begin{tabular}{lrrrrr} 
Source & \multicolumn{1}{c}{ SS } & DF & \multicolumn{1}{c}{ MS } & $\underline{F}$ & $\underline{\text { F }}$ \\
Within Cells & 87.00 & 39 & 2.23 & & \\
Regression & 21.49 & 1 & 21.49 & 9.63 & .004 \\
Constant & 43.75 & 1 & 43.75 & 19.61 & .000 \\
Treatment & 7.39 & 1 & 7.39 & 3.31 & .076 \\
Condition & .32 & 1 & .32 & .14 & .708 \\
Treatment by condition & 15.75 & 1 & 15.75 & 7.06 & .011
\end{tabular}

Variable 3: Social Adjustment Scale - Family of origin

Source

Within cells

Regression

Constant

Treatment

condition

Treatment by condition
SS $\quad \underline{D F}$

50.06

36.53

4.45

6.47

.19

.13
38

1

1

1

1

1
MS

1.32

36.53

4.45

6.47

.19

.13

\section{$\underline{\mathbf{F}} \quad \underline{\mathrm{p}}$}

$27.73 \quad .000$

$3.38 \quad .074$

$4.91 \quad .033$

$.14 \quad .706$

$.10 \quad .753$

Variable 4: Social Adjustment Scale - Partner/Spouse

Source

Within Cells

Regression

Constant

Treatment

condition

Treatment by condition

\begin{tabular}{rr}
\multicolumn{1}{l}{ SS } & $\underline{\mathrm{DF}}$ \\
17.55 & 9 \\
39.45 & 1 \\
.04 & 1 \\
6.69 & 1 \\
.07 & 1 \\
.04 & 1
\end{tabular}

$\underline{\text { MS }}$

$\underline{F}$

$\underline{\underline{P}}$

1.95

39.45

.04

6.69

.07

.04

$\begin{array}{rr}20.23 & .001 \\ .02 & .885 \\ 3.43 & .097 \\ .03 & .856 \\ .02 & .890\end{array}$


Variable 5: Social Adjustment Scale - Sexual Iife

\begin{tabular}{lrrrrr} 
Source & \multicolumn{1}{c}{ SS } & DF & \multicolumn{1}{c}{ MS } & $\underline{F}$ & $\underline{P}$ \\
Within Cells & 311.89 & 39 & 8.00 & & \\
Regression & 295.40 & 1 & 295.40 & 36.94 & .000 \\
Constarnt & 50.50 & 1 & 50.50 & 6.31 & .016 \\
Treatment & 80.81 & 1 & 80.81 & 10.11 & .003 \\
Condition & 19.06 & 1 & 19.06 & 2.38 & .131 \\
Treatment by condition & 8.04 & 1 & 8.04 & 1.01 & .322
\end{tabular}

Variable 6: Social Adjustment scale - Children

\begin{tabular}{lrrrrr} 
Source & \multicolumn{1}{c}{ SS } & DF & \multicolumn{1}{c}{ MS } & $\underline{F}$ & $\underline{\text { P }}$ \\
Within Cells & 52.11 & 26 & 2.00 & & \\
Regression & 58.07 & 1 & 58.07 & 28.97 & .000 \\
Constant & 2.00 & 1 & 2.00 & 1.00 & .327 \\
Treatment & .85 & 1 & .85 & .42 & .521 \\
Condition & .00 & 1 & .00 & .00 & .993 \\
Treatment by condition & 2.09 & 1 & 2.09 & 1.04 & .316
\end{tabular}

Variable 7: Target Severity - Independently-rated

\begin{tabular}{lrrrrr} 
Source & \multicolumn{1}{c}{ SS } & DF & NS & F & P \\
Within Cells & 44.96 & 39 & 1.15 & & \\
Regression & 3.95 & 1 & 3.95 & 3.43 & .072 \\
Constant & .64 & 1 & .64 & .55 & .462 \\
Treatment & 11.47 & 1 & 11.47 & 9.95 & .003 \\
Condition & 3.77 & 1 & 3.77 & 3.27 & .078 \\
Treatment by condition & 2.81 & 1 & 2.81 & 2.44 & .127
\end{tabular}

Variable 8: Target Severity - Patient-rated

$\begin{array}{lrrrrr}\text { Source } & \text { SS } & \text { DF } & \text { MS } & \text { F } & \underline{P} \\ \text { Within cells } & 46.71 & 39 & 1.20 & & \\ \text { Regression } & 3.21 & I & 3.21 & 2.68 & .110 \\ \text { Constant } & 3.20 & 1 & 3.20 & 2.67 & .110 \\ \text { Treatnent } & 2.76 & 1 & 2.76 & 2.31 & .137 \\ \text { Condition } & .19 & 1 & .19 & .16 & .691 \\ \text { Treatment by condition } & 1.63 & 1 & 1.63 & 1.36 & .250\end{array}$


Variable 9: Impact of Event Scale - Intrusion

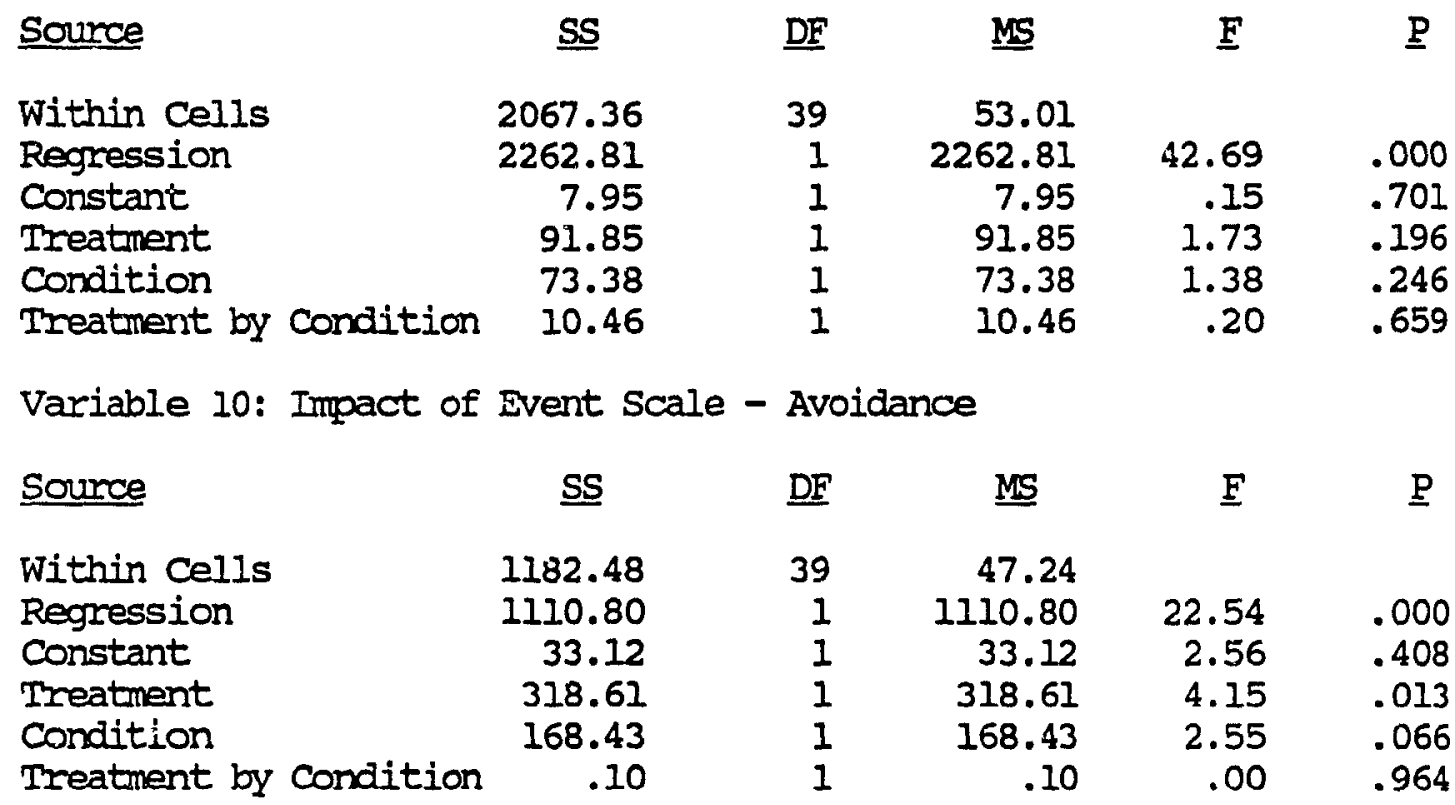

Variable 11: Interpersonal Behavior Scale - Present Functioning

$\begin{array}{lrrrrr}\text { Source } & \text { SS } & \text { DF } & \text { MS } & \underline{F} & \underline{\text { N }} \\ \text { Within Cells } & 9502.94 & 39 & 243.67 & & \\ \text { Regression } & 9536.07 & 1 & 9536.07 & 39.14 & .000 \\ \text { Constant } & 273.16 & 1 & 273.16 & 1.12 & .296 \\ \text { Treatment } & 1249.08 & 1 & 1249.08 & 5.13 & .029 \\ \text { Condition } & 13.02 & 1 & 13.02 & .05 & .818 \\ \text { Treatment by condition } & 1394.60 & 1 & 1394.60 & 5.72 & .022\end{array}$

Variable 12: Interpersomal Dependency Inventory - Emotional Reliance

\begin{tabular}{lrrrrr} 
Source & \multicolumn{1}{l}{ SS } & DF & \multicolumn{1}{l}{ MS } & $\underline{F}$ & $\underline{P}$ \\
Within Cells & 4.83 & 38 & .13 & & \\
Regression & 9.41 & 1 & 9.41 & 73.99 & .000 \\
Constant & .32 & 1 & .32 & 2.50 & .122 \\
Treatment & .02 & 1 & .02 & .17 & .680 \\
Condition & .28 & 1 & .28 & 2.22 & .145 \\
Treatment by condition & .01 & 1 & .01 & .10 & .754
\end{tabular}


Variable 13: Interpersonal Dependency Inventory - Autonomy

\begin{tabular}{lrrrrr} 
Source & \multicolumn{1}{l}{ SS } & DF & MS & F & $\underline{\text { P }}$ \\
Within Cells & 2.67 & 38 & .07 & & \\
Regression & 3.98 & 1 & 3.98 & 56.57 & .000 \\
Constant & .51 & 1 & .51 & 7.19 & .011 \\
Treatment & .33 & 1 & .33 & 4.74 & .036 \\
Condition & .04 & 1 & .04 & .52 & .474 \\
Treatment by condition & .06 & 1 & .06 & .90 & .348
\end{tabular}

Variable 14: SCI-90 - Total Score

$\begin{array}{lrrrrr}\text { Source } & \text { SS } & \text { DF } & \text { MS } & \underline{F} & \underline{P} \\ \text { Within Cells } & 65448.11 & 33 & 1983.28 & & \\ \text { Regression } & 17906.03 & 1 & 17906.03 & 9.03 & .005 \\ \text { Constant } & 6404.94 & 1 & 6404.94 & 3.23 & .081 \\ \text { Treatment } & 20623.37 & 1 & 20623.37 & 10.40 & .003 \\ \text { Condition } & 97.03 & 1 & 97.03 & .05 & .826 \\ \text { Treatment by condition } 9959.02 & 1 & 9959.02 & 5.02 & .032\end{array}$

Variable 15: Beck Depression Inventory

\begin{tabular}{lrrrrr} 
Source & \multicolumn{1}{c}{ SS } & DF & MS & $\underline{F}$ & $\underline{P}$ \\
Within Cells & 557.47 & 39 & 14.29 & & \\
Regression & 571.69 & 1 & 571.69 & 39.99 & .000 \\
Constant & 31.11 & 1 & 31.11 & 2.18 & .148 \\
Treatment & 147.47 & 1 & 147.47 & 10.32 & .003 \\
Condition & 14.57 & 1 & 14.57 & 1.02 & .319 \\
Treatment by condition & 9.31 & 1 & 9.31 & .65 & .425
\end{tabular}

Variable 16: Rosenberg Self-esteem Scale

\begin{tabular}{lrrrrr} 
Scurce & \multicolumn{1}{c}{ SS } & DF & \multicolumn{1}{c}{ MS } & $\underline{F}$ & $\underline{P}$ \\
Within cells & 75.71 & 36 & 2.10 & & \\
Regression & 57.80 & 1 & 57.80 & 27.48 & .000 \\
Consiant & 2.18 & 1 & 2.18 & 1.04 & .315 \\
Treatuent & 35.52 & 1 & 35.52 & 16.89 & .000 \\
Condition & 10.29 & 1 & 10.29 & 4.89 & .033 \\
Treatment by condition & 7.50 & 1 & 7.50 & 3.57 & .067
\end{tabular}


Variable 17: Iife Satisfaction Scile

\begin{tabular}{lrrrrr} 
Source & \multicolumn{1}{c}{ SS } & DF & MS & F & $\underline{\underline{P}}$ \\
Within Cells & 52.79 & 36 & 1.47 & & \\
Regression & 8.98 & 1 & 8.98 & 6.12 & .018 \\
Constant & 64.71 & 1 & 64.71 & 44.13 & .000 \\
Treatment & 14.45 & 1 & 14.45 & 9.85 & .003 \\
Condition & 5.06 & 1 & 5.06 & 3.45 & .071 \\
Treatment by condition & 1.78 & 1 & 1.78 & 1.21 & .278
\end{tabular}


APPENDIX P: Mean and Standard Deviation of EWORS Variables

FMORS Variables

Participation

Self-based

Work.

43

43

43

43

Group-based

Work

High-level

Group-based

Work

43
Mean
18.5

31.2

8.9

7.8

18.8

10.9

18.9

12.0 